\title{
Diastereo- and Enantioselective 1,6-Conjugate Addition of 2- Azaarylacetamides to para-Quinone Methides
}

\author{
Yan Wang, Kaixuan Wang, Weidi Cao*, Xiaohua Liu, Xiaoming Feng* \\ Key Laboratory of Green Chemistry \& Technology, Ministry of Education, College of Chemistry, \\ Sichuan University, Chengdu 610064, People's Republic of China
}

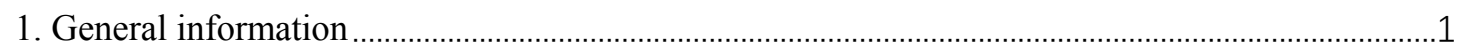

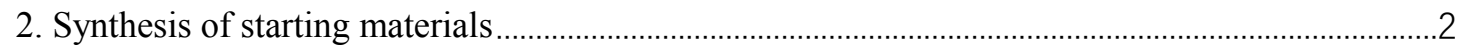

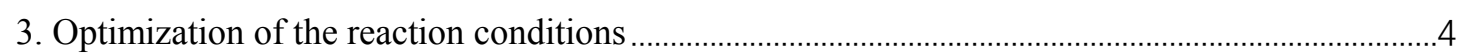

4. General procedure for the catalytic asymmetric reaction..............................................................

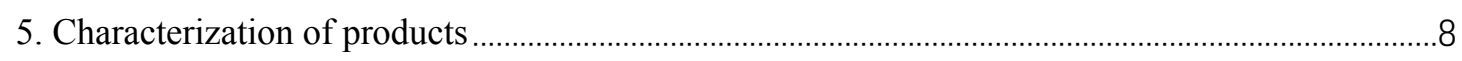

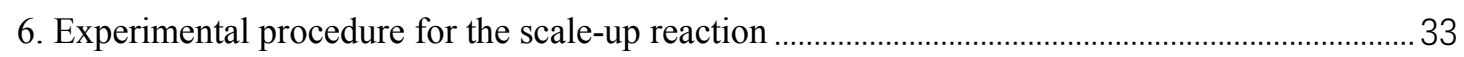

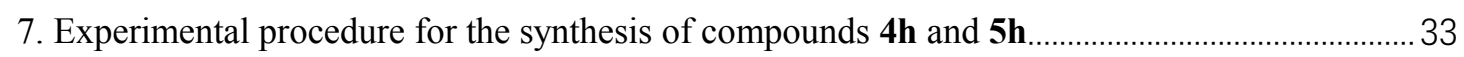

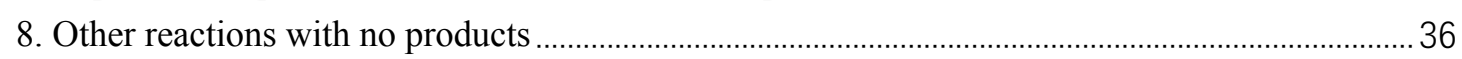

9. Demonstration of reaction mechanism by HRMS and control experiment ....................................36

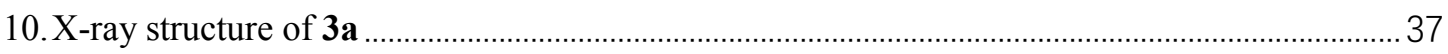

11. Reference

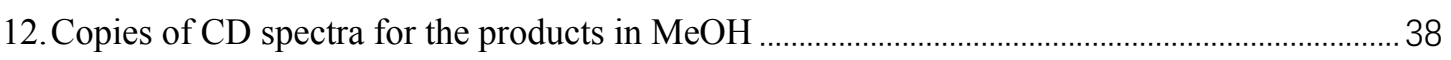

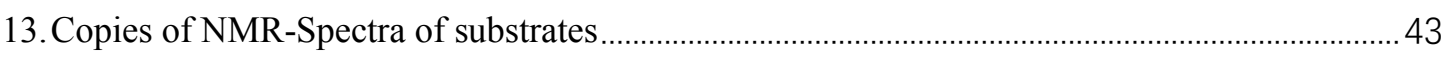

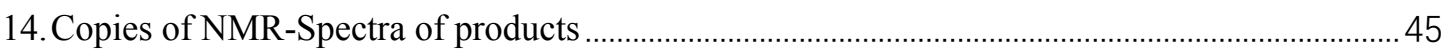

\section{General information}

${ }^{1}$ H NMR spectra were recorded on commercial instruments (400 MHz). Chemical shifts were reported in ppm from tetramethylsilane with the solvent resonance as the internal standard $\left(\mathrm{CDCl}_{3}\right.$, $\delta=7.26$ ). Spectra were reported as follows: chemical shift $(\delta \mathrm{ppm})$, multiplicity ( $\mathrm{s}=$ singlet, $\mathrm{d}=$ doublet), coupling constants (Hz), integration and assignment.

${ }^{13} \mathrm{C}$ NMR spectra were collected on commercial instruments $(100 \mathrm{MHz})$ with complete proton decoupling. Chemical shifts are reported in ppm from the tetramethylsilane with the solvent resonance as internal standard $\left(\mathrm{CDCl}_{3}, \delta=77.0\right)$.

${ }^{19}$ F NMR spectra were collected on commercial instruments $(376 \mathrm{MHz})$.

Enantiomeric excesses (ee) were determined by HPLC analysis using the corresponding commercial chiralpak column as stated in the experimental procedures at $25{ }^{\circ} \mathrm{C}$.

Optical rotations were reported as follows: $[\alpha]_{\mathrm{D}}^{25}(c: \mathrm{g} / 100 \mathrm{~mL}$, in solvent).

HRMS was recorded on a commercial apparatus (ESI Source).

Solvents: THF was freshly distilled from sodium metal prior to use. $\mathrm{CH}_{2} \mathrm{Cl}_{2}$ was freshly distilled from $\mathrm{CaH}_{2}$ prior to use.

Racemic products: all racemic products were obtained by using $\mathrm{Ni}(\mathrm{OTf})_{2} /( \pm)-\mathbf{L}_{3} \mathbf{P i E t}_{2}(2 \mathrm{~mol} \%)$, $\mathrm{K}_{2} \mathrm{CO}_{3}(50 \mathrm{~mol} \%)$ at $35^{\circ} \mathrm{C}$ for $24 \mathrm{~h}$. 
Metal salts source: $\operatorname{Mg}(\mathrm{OTf})_{2}$ was purchased from Alfa without further purification.

\section{Synthesis of starting materials}

\section{a) Synthesis of $\alpha$-Azaarylamides}<smiles>CNC(=O)Cc1nc2ccccc2s1</smiles>

$1 \mathrm{a}$<smiles>CNC(=O)Cc1nc2ccccc2o1</smiles>

$1 b$<smiles>CNC(=O)Cc1ccc(Br)cn1</smiles>

$1 c$<smiles>CN(C)C(=O)Cc1nccnc1Cl</smiles>

$1 d$

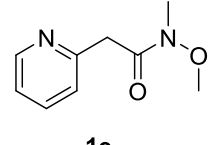

1e<smiles>C=CCN(C)C(=O)Cc1ccccn1</smiles>

$1 f$<smiles>O=C(Cc1ccccn1)N1CCOCC1</smiles>

$1 \mathrm{~g}$<smiles>CC(C)N(C(=O)Cc1nc2ccccc2s1)C(C)C</smiles>

$1 \mathrm{~h}$<smiles>CC1(C)CCCC(C)(C)N1C(=O)Cc1nc2ccccc2s1</smiles>

1a and $\mathbf{1 c}$ were synthesized following a published procedure. ${ }^{1} \mathbf{1 b}, \mathbf{1 d}, \mathbf{1 e}, \mathbf{1 f}$ and $\mathbf{1 g}$ were synthesized following a published procedure. ${ }^{2}$ It failed to synthesize $\mathbf{1 h}$ and $\mathbf{1 i}$.

b) Synthesis of $p$-Quinone methides

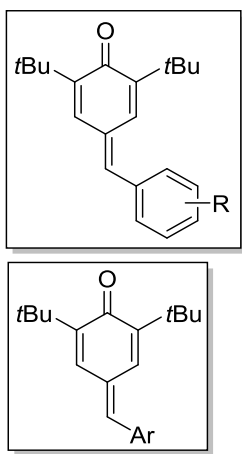

2a: $R=H, 2 b: R=4-F, 2 c: R=4-C l$

2d: $R=4-B r, 2 e: R=4-I, 2 f: R=4-C N$

2g: $R=4-M e, 2 h: R=4-O M e$

2i: $R=3-\mathrm{Cl}, 2 \mathrm{j}: \mathrm{R}=2-\mathrm{Cl}, \mathbf{2 k}: \mathrm{R}=2-\mathrm{CF}_{3}$,
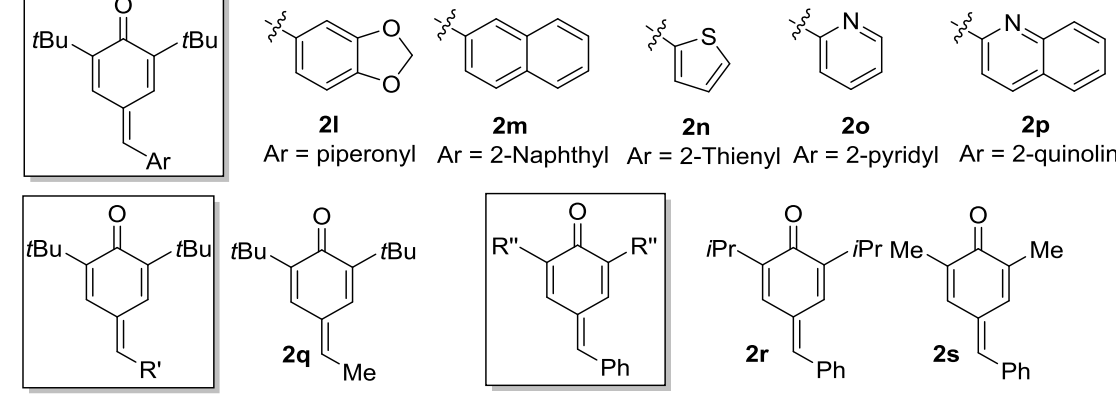

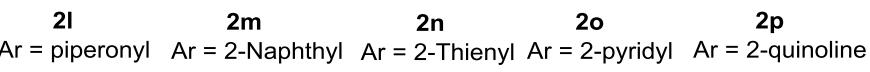

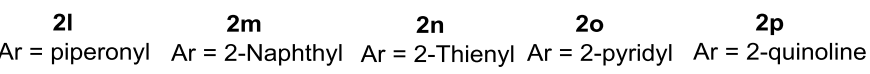

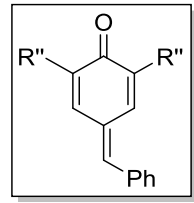



2a, 2c, 2d, 2g, $\mathbf{2 h}, \mathbf{2}, \mathbf{2 m}$ and $\mathbf{2 s}$ were synthesized following a published procedure. ${ }^{3} \mathbf{2 f}, \mathbf{2 i}, \mathbf{2} \mathbf{j}$ and $\mathbf{2 k}$ were synthesized following a published procedure. ${ }^{4} \mathbf{2 b}$ and $\mathbf{2 n}$ were synthesized following a published procedure..$^{5} \mathbf{2 0}$ was synthesized following a published procedure. ${ }^{6} \mathbf{2 q}$ and $\mathbf{2 r}$ were synthesized following a published procedure. ${ }^{7}$

$\mathbf{2 e}$ and $\mathbf{2 p}$ were synthesized by the following procedure:

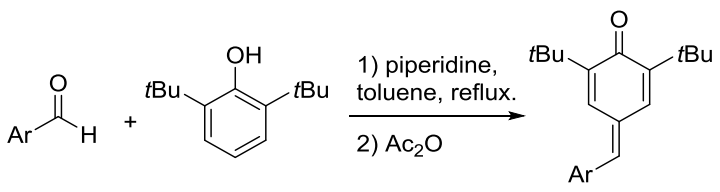

According to literature, ${ }^{3-6} 1.0$ equiv of 2,6-di-t-butylphenol (10.0 mmol, $\left.2.06 \mathrm{~g}\right)$ and 1.0 equiv of aldehyde $(10.0 \mathrm{mmol})$ were dissolved in toluene $(50 \mathrm{~mL})$ and heated to reflux in a Dean-Stark apparatus. Piperidine ( 2.0 equiv, $2.0 \mathrm{~mL}$ ) was added dropwise over $1 \mathrm{~h}$ and the reaction mixture was refluxed for $3 \mathrm{~h}$. After cooling just below the boiling point of the mixture, acetic anhydride (2.0 equiv, $2.0 \mathrm{~mL}$ ) was added and stirring continued for $15 \mathrm{~min}$. Then the reaction mixture was poured on ice-water, extracted with DCM (3 times), dried over $\mathrm{Na}_{2} \mathrm{SO}_{4}$, the solvent evaporated and the residue dried in vacuo. The resulting crude product was subjected to column chromatography 
(petroleum ether/ ethyl acetate $=50: 1$ ).

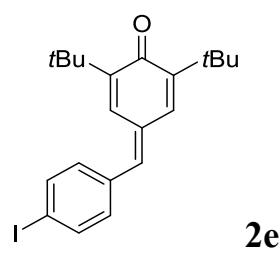

\section{2,6-di-tert-butyl-4-(4-iodobenzylidene)cyclohexa-2,5-dien-1-one}

$2 \%$ to $10 \%$ EtOAc in hexanes was used for flash column chromatography. The title compound was isolated (1.60 g, 38\% yield) as orange solid;

${ }^{1}$ H NMR (400 MHz, Chloroform- $d$ ) $\delta 7.82-7.76(\mathrm{~m}, 2 \mathrm{H}), 7.43-7.42(\mathrm{~m}, 1 \mathrm{H}), 7.20-7.15(\mathrm{~m}$, 2H), 7.07 (s, 1H), $6.99-6.98(\mathrm{~m}, 1 \mathrm{H}), 1.32(\mathrm{~s}, 9 \mathrm{H}), 1.29(\mathrm{~s}, 9 \mathrm{H})$;

${ }^{13}$ C NMR (101 MHz, Chloroform- $d$ ) $\delta 186.5,149.7,148.1,140.8,138.0,135.3,134.8,132.4,131.8$, 127.2, 35.5, 35.0, 29.5, 29.4;

HRMS (ESI) scaled for $[\mathrm{M}+\mathrm{H}]^{+}: \mathrm{C}_{21} \mathrm{H}_{26} \mathrm{IO}, \mathrm{m} / \mathrm{z}$ : 421.1023, observed: 421.1028;

m.p. $=170{ }^{\circ} \mathrm{C}$.

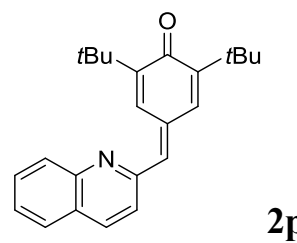

\section{2,6-di-tert-butyl-4-(quinolin-2-ylmethylene)cyclohexa-2,5-dien-1-one}

$2 \%$ to $10 \%$ EtOAc in hexanes was used for flash column chromatography. The title compound was isolated (207 $\mathrm{mg}, 6 \%$ yield) as orange solid;

${ }^{1}$ H NMR (400 MHz, Chloroform- $d$ ) $\delta 9.04-9.03(\mathrm{~m}, 1 \mathrm{H}), 8.19-8.17(\mathrm{~m}, 1 \mathrm{H}), 8.12-8.09(\mathrm{~m}, 1 \mathrm{H})$, $7.83-7.81(\mathrm{~m}, 1 \mathrm{H}), 7.79-7.71(\mathrm{~m}, 1 \mathrm{H}), 7.59-7.56(\mathrm{~m}, 1 \mathrm{H}), 7.51-7,49(\mathrm{~m}, 1 \mathrm{H}), 7.09(\mathrm{~s}, 1 \mathrm{H}), 7.03-$ $7.02(\mathrm{~m}, 1 \mathrm{H}), 1.39$ (s, 9H), $1.34(\mathrm{~s}, 9 \mathrm{H})$;

${ }^{13}$ C NMR (101 MHz, Chloroform- $d$ ) $\delta$ 186.7, 155.1, 150.2, 148.9, 148.4, 137.8, 136.3, 135.8, 135.3, 130.2, 129.9, 129.6, 127.5, 127.3, 126.9, 124.4, 35.8, 35.1, 29.6, 29.6;

HRMS (ESI) scaled for $[\mathrm{M}+\mathrm{H}]^{+}: \mathrm{C}_{24} \mathrm{H}_{28} \mathrm{NO}, \mathrm{m} / \mathrm{z}: 346.2165$, observed: 346.2169;

m.p. $=160{ }^{\circ} \mathrm{C}$. 


\section{Optimization of the reaction conditions}

Table S1. The screening of the metal salts for the reaction between 1a and $\mathbf{2 a}$.

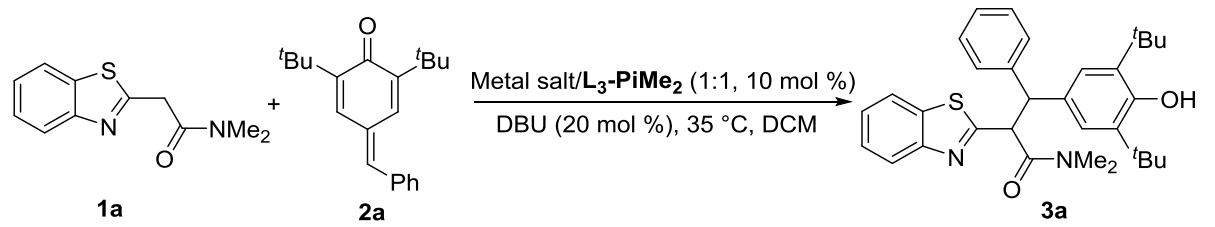

$1 \mathbf{a}$

3a

\begin{tabular}{ccccc}
\hline entry $^{a}$ & Metal salt & ${\text { Yield }(\%)^{b}}^{b}$ & $\mathrm{dr}^{c}$ & ee $(\%)^{c}$ \\
\hline 1 & $\mathrm{Fe}(\mathrm{OTf})_{3}$ & 35 & $80: 20$ & $84 / 68$ \\
2 & $\mathrm{Fe}(\mathrm{OTf})_{2}$ & 97 & $48: 52$ & $21 / 3$ \\
3 & $\mathrm{Co}\left(\mathrm{ClO}_{4}\right)_{2} \cdot 6 \mathrm{H}_{2} \mathrm{O}$ & 93 & $49: 51$ & $91 / 93$ \\
4 & $\mathrm{Ni}(\mathrm{OTf})_{2}$ & 99 & $82: 18$ & $18 / 49$ \\
5 & $\mathrm{Cu}(\mathrm{OTf})_{2}$ & 55 & $55: 45$ & $6 / 17$ \\
6 & $\mathrm{Zn}(\mathrm{OTf})_{2}$ & 99 & $80: 20$ & $2 / 2$ \\
7 & $\mathrm{Sc}(\mathrm{OTf})_{3}$ & 77 & $58: 42$ & $65 / 35$ \\
8 & $\mathrm{Y}(\mathrm{OTf})_{3}$ & 98 & $44: 56$ & $3 / 2$ \\
9 & $\mathrm{La}(\mathrm{OTf})_{3}$ & 92 & $50: 50$ & $27 / 5$ \\
10 & $\mathrm{Lu}(\mathrm{OTf})_{3}$ & 86 & $45: 55$ & $5 / 1$ \\
11 & $\mathrm{Mg}(\mathrm{OTf})_{2}$ & 99 & $62: 38$ & $90 / 72$ \\
12 & $\mathrm{Ca}(\mathrm{OTf})_{2}$ & 99 & $53: 47$ & $52 /-22$ \\
13 & $\mathrm{Ba}(\mathrm{OTf})_{2}$ & 99 & $48: 52$ & race \\
\hline
\end{tabular}

${ }^{a}$ All reactions were performed with $\mathbf{1 a}(0.1 \mathrm{mmol}), \mathbf{2 a}(0.1 \mathrm{mmol})$, metal salt/ $\mathbf{L} 3-\mathrm{PiMe}_{2}(1: 1,10 \mathrm{~mol} \%)$, DBU $(20 \mathrm{~mol} \%)$, in DCM $(1.0 \mathrm{~mL})$ at $35{ }^{\circ} \mathrm{C}$ for 20 hours. ${ }^{b}$ Isolated yield. ${ }^{c}$ The $\mathrm{dr}$ and ee were determined by ${ }^{1} \mathrm{H}$ NMR analysis and chiral HPLC analysis, respectively.

Table S2. The screening of the base for the reaction between 1a and $2 \mathbf{a}$.

\begin{tabular}{|c|c|c|c|c|}
\hline entry $^{a}$ & Base & Yield $(\%)^{b}$ & $\mathrm{dr}^{c}$ & ee $(\%)^{c}$ \\
\hline 14 & $\mathrm{DBU}$ & 99 & $62: 38$ & $90 / 72$ \\
\hline 15 & Imidazole & NR & / & I \\
\hline 16 & $\mathrm{Et}_{3} \mathrm{~N}$ & 60 & $61: 39$ & $97: 85$ \\
\hline 17 & pyridine & NR & / & / \\
\hline 18 & $\mathrm{~K}_{2} \mathrm{CO}_{3}$ & 99 & $68: 32$ & $97 / 85$ \\
\hline 19 & I & NR & l & l \\
\hline
\end{tabular}

${ }^{a}$ All reactions were performed with $\mathbf{1 a}(0.1 \mathrm{mmol}), \mathbf{2 a}(0.1 \mathrm{mmol}), \mathbf{M g}(\mathrm{OTf})_{2} / \mathbf{L}_{3}-\mathrm{PiMe}_{2}(1: 1,10 \mathrm{~mol} \%)$, base $(20 \mathrm{~mol} \%)$, in DCM $(1.0 \mathrm{~mL})$ at $35{ }^{\circ} \mathrm{C}$ for 20 hours. ${ }^{b}$ Isolated yield. ${ }^{C}$ The $\mathrm{dr}$ and ee were determined by ${ }^{1} \mathrm{H}$ NMR analysis and chiral HPLC analysis, respectively.

Table S3. The screening of ligands for the reaction between 1a and $2 \mathbf{a}$. 


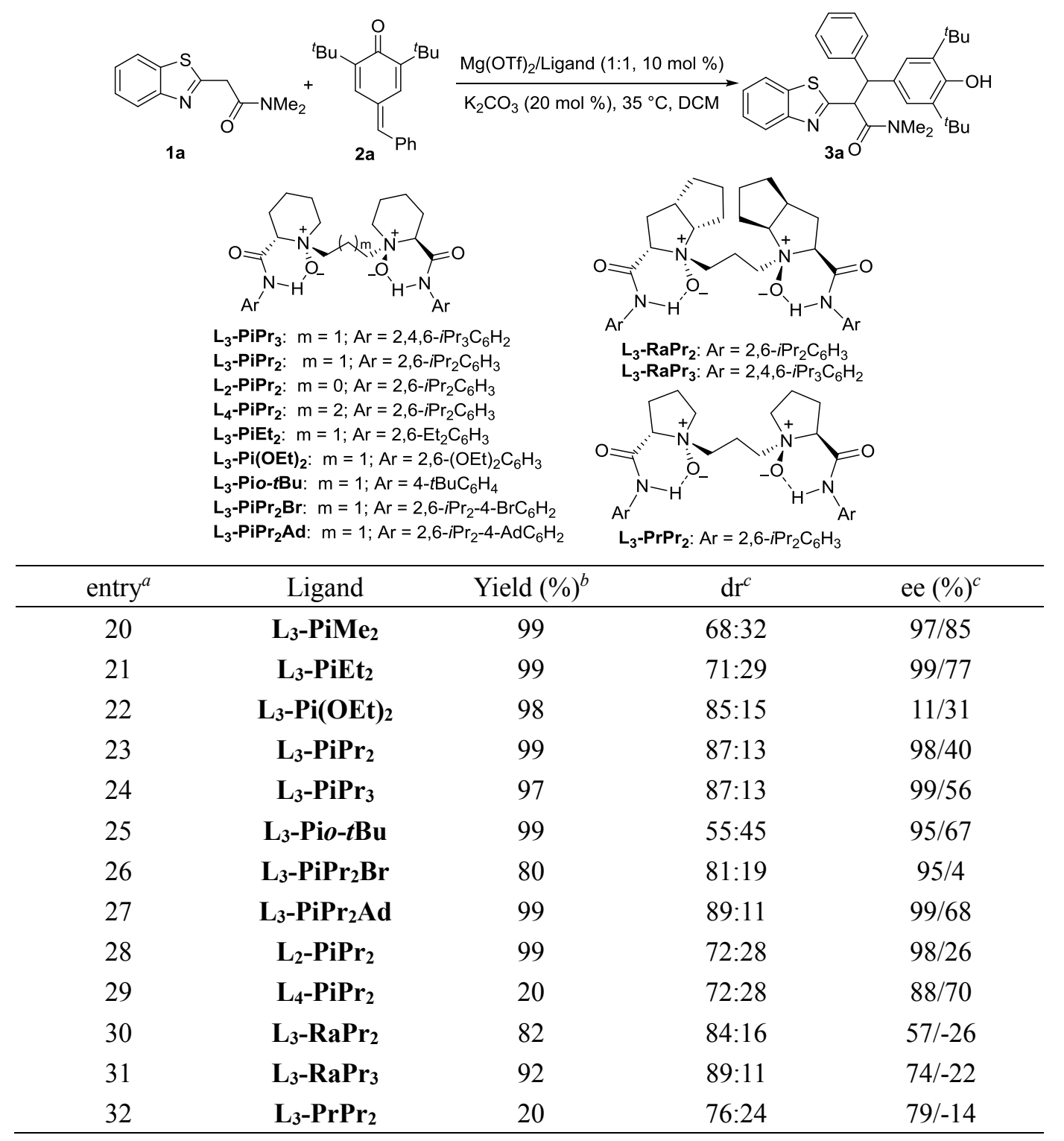

${ }^{a}$ All reactions were performed with $\mathbf{1 a}(0.1 \mathrm{mmol}), \mathbf{2 a}(0.1 \mathrm{mmol}), \mathrm{Mg}(\mathrm{OTf}) / \mathrm{Ligand}(1: 1,10 \mathrm{~mol} \%), \mathrm{K}_{2} \mathrm{CO}_{3}(20 \mathrm{~mol} \%)$, in DCM $(1.0 \mathrm{~mL})$ at $35^{\circ} \mathrm{C}$ for 20 hours. ${ }^{b}$ Isolated yield. ${ }^{\top}$ The $\mathrm{dr}$ and ee were determined by ${ }^{1} \mathrm{H}$ NMR analysis and chiral HPLC analysis, respectively.

Table S4. The screening of the temperature for the reaction between 1a and 2a.

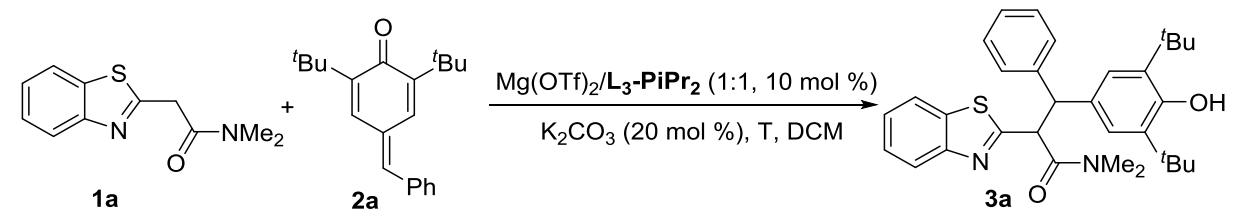

\begin{tabular}{ccccc}
\hline entry $^{a}$ & $\mathrm{~T}$ & ${\text { Yield }(\%)^{b}}^{b}$ & $\mathrm{dr}^{c}$ & ee (\%) \\
\hline 33 & $35^{\circ} \mathrm{C}$ & 99 & $87: 13$ & $98 / 40$ \\
34 & $20^{\circ} \mathrm{C}$ & 82 & $89: 11$ & $98 / 50$ \\
35 & $0{ }^{\circ} \mathrm{C}$ & 69 & $95: 5$ & $98 / 44$ \\
36 & $-20{ }^{\circ} \mathrm{C}$ & trace & $/$ & $/$ \\
\hline
\end{tabular}

${ }^{a}$ All reactions were performed with $\mathbf{1 a}(0.1 \mathrm{mmol}), \mathbf{2 a}(0.1 \mathrm{mmol}), \mathrm{Mg}(\mathrm{OTf})_{2} / \mathbf{L}_{3}-\mathrm{PiPr}_{2}(1: 1,10 \mathrm{~mol} \%), \mathrm{K}_{2} \mathrm{CO}_{3}(20 \mathrm{~mol} \%)$, in DCM $(1.0 \mathrm{~mL})$ at different temperature for 20 hours. ${ }^{b}$ Isolated yield. ${ }^{c}$ The $\mathrm{dr}$ and ee were determined by ${ }^{1} \mathrm{H}$ NMR analysis and chiral HPLC analysis, respectively.

Table S5. The screening of the solvent for the reaction between 1a and $\mathbf{2 a}$. 


\begin{tabular}{|c|c|c|c|c|}
\hline entry ${ }^{a}$ & solvent & Yield $(\%)^{b}$ & $\mathrm{dr}^{\mathrm{c}}$ & ee $(\%)^{\mathrm{c}}$ \\
\hline 37 & DCM & 99 & $87: 13$ & $98 / 40$ \\
\hline 38 & $\mathrm{CH}_{3} \mathrm{Cl}$ & trace & / & / \\
\hline 39 & $\mathrm{CH}_{2} \mathrm{ClCH}_{2} \mathrm{Cl}$ & 99 & $87: 13$ & $98 / 50$ \\
\hline 40 & EA & 99 & $90: 10$ & $94 / 20$ \\
\hline 41 & ether & 60 & $50 / 50$ & $76 / 14$ \\
\hline 42 & MTBE & 90 & $90 / 10$ & $91 / 30$ \\
\hline 43 & THF & 99 & $91: 9$ & $98 / 28$ \\
\hline $44^{\mathrm{d}}$ & THF & 99 & $93: 7$ & $99 / 32$ \\
\hline $45^{\mathrm{e}}$ & THF & 99 & $93: 7$ & $99 / 32$ \\
\hline 46 & $\mathrm{CH}_{3} \mathrm{CN}$ & 99 & $84: 16$ & $97 / 30$ \\
\hline 47 & toluene & 99 & $78: 22$ & $98 / 42$ \\
\hline
\end{tabular}

${ }^{\mathrm{a}} \mathrm{All}$ reactions were performed with $\mathbf{1 a}(0.1 \mathrm{mmol}), \mathbf{2 a}(0.1 \mathrm{mmol}), \mathrm{Mg}(\mathrm{OTf})_{2} / \mathbf{L}_{3}-\mathbf{P i P r}_{2}(1: 1,10 \mathrm{~mol} \%), \mathrm{K}_{2} \mathrm{CO}_{3}(20 \mathrm{~mol} \%)$, in solvent $(1.0 \mathrm{~mL})$ at 35 ${ }^{\circ} \mathrm{C}$ for 20 hours. ${ }^{b}$ Isolated yield. ${ }^{\circ} \mathrm{The} \mathrm{dr}$ and ee were determined by ${ }^{1} \mathrm{H}$ NMR analysis and chiral HPLC analysis, respectively. ${ }^{\mathrm{d}}$ Reacted at $0{ }^{\circ} \mathrm{C}$ for $24 \mathrm{~h}$. ${ }^{\mathrm{e}}$ The reaction was performed with $\mathbf{1 a}(0.1 \mathrm{mmol}), \mathbf{2 a}(0.1 \mathrm{mmol}), \mathrm{Mg}(\mathrm{OTf})_{2} / \mathbf{L}_{3}-\mathbf{P i P r}_{2}(1: 1,5 \mathrm{~mol} \%), \mathrm{K}_{2} \mathrm{CO}_{3}(10 \mathrm{~mol} \%)$, in THF $(1.0 \mathrm{~mL})$ at 0 ${ }^{\circ} \mathrm{C}$ for 24 hours.

Table S6. The screening of the reaction conditions for the reaction between $1 \mathbf{c}$ and $\mathbf{2 a}$.

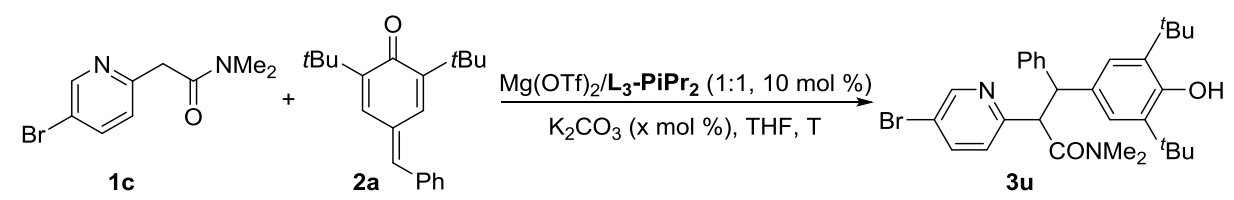

\begin{tabular}{ccccc}
\hline entry $^{a}$ & conditions & ${\text { Yield }(\%)^{b}}^{b}$ & $\mathrm{dr}^{c}$ & ee (\%) \\
\hline 48 & $0{ }^{\circ} \mathrm{C}, \mathrm{K}_{2} \mathrm{CO}_{3}(20 \mathrm{~mol} \mathrm{\% )}$ & 56 & $79: 21$ & $87 / 85$ \\
49 & $35{ }^{\circ} \mathrm{C}, \mathrm{K}_{2} \mathrm{CO}_{3}(20 \mathrm{~mol} \%)$ & 77 & $78: 22$ & $97 / 75$ \\
50 & $35{ }^{\circ} \mathrm{C}, \mathrm{K}_{2} \mathrm{CO}_{3}(50 \mathrm{~mol} \%)$ & 93 & $78: 22$ & $96 / 57$ \\
51 & $45^{\circ} \mathrm{C}, \mathrm{K}_{2} \mathrm{CO}_{3}(50 \mathrm{~mol} \%)$ & 76 & $80: 20$ & $95 / 62$ \\
$52^{\mathrm{d}}$ & $0{ }^{\circ} \mathrm{C}, \mathrm{K}_{2} \mathrm{CO}_{3}(20 \mathrm{~mol} \mathrm{\% )}$ & trace & $/$ & $/$ \\
\hline
\end{tabular}

${ }^{a}$ All reactions were performed with $\mathbf{1 d}(0.1 \mathrm{mmol}), \mathbf{2 a}(0.1 \mathrm{mmol}), \mathrm{Mg}(\mathrm{OTf})_{2} / \mathbf{L}_{3}-\mathrm{PiPr}_{2}(1: 1,10 \mathrm{~mol} \%), \mathrm{K}_{2} \mathrm{CO}_{3}(\mathrm{x} \mathrm{mol} \%)$, in THF $(1.0 \mathrm{~mL})$ for 36 hours. ${ }^{b}$ Isolated yield. ${ }^{c} \mathrm{The} \mathrm{dr}$ and ee were determined by ${ }^{1} \mathrm{H}$ NMR analysis and chiral HPLC analysis, respectively. ${ }^{\mathrm{d}}$ The reaction was performed with 1f $(0.1 \mathrm{mmol}), \mathbf{2 a}(0.1 \mathrm{mmol}), \mathrm{Mg}(\mathrm{OTf})_{2} / \mathbf{L}_{3}-\operatorname{PiPr}_{2}(1: 1,5 \mathrm{~mol} \%), \mathrm{K}_{2} \mathrm{CO}_{3}(20 \mathrm{~mol} \%)$, in THF $(1.0 \mathrm{~mL})$ at $0{ }^{\circ} \mathrm{C}$ for 36 hours. 


\section{General procedure for the catalytic asymmetric reaction}

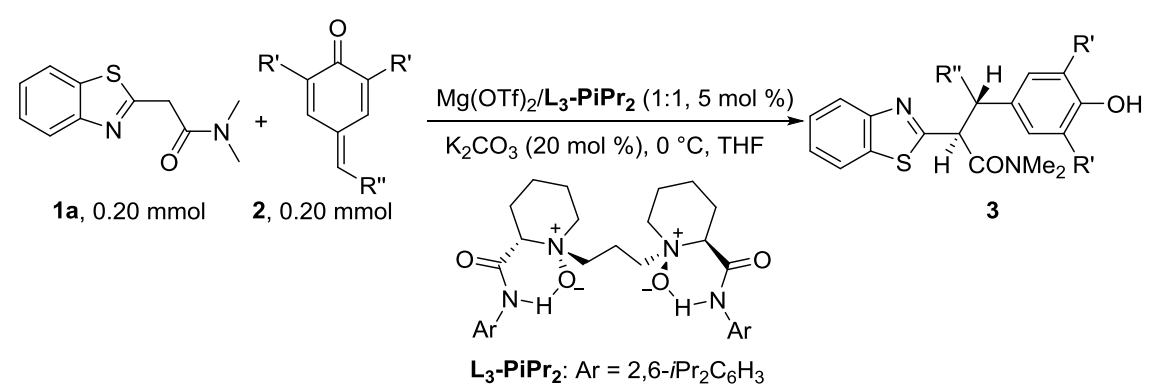

1a $(0.20 \mathrm{mmol}), \mathrm{Mg}(\mathrm{OTf})_{2}(0.01 \mathrm{mmol}, 3.2 \mathrm{mg}), \mathbf{L}_{3}-\mathbf{P i P r}_{2}(0.01 \mathrm{mmol}, 6.5 \mathrm{mg})$ and $\mathrm{K}_{2} \mathrm{CO}_{3}(0.04$ $\mathrm{mmol}, 5.5 \mathrm{mg})$ were weighed into the test tube. THF $(1.0 \mathrm{~mL})$ was added, then the reaction mixture was stirred at $35{ }^{\circ} \mathrm{C}$ for 30 minutes to ensure a better coordination between the metal salt and the ligand. Then the reaction was transferred to $0{ }^{\circ} \mathrm{C}$, after a few minutes, $2(0.20 \mathrm{mmol})$ was added to the test tube. The mixture was stirred and detected by TLC (petroleum ether/ ethyl acetate $=3: 1$ ). After completion, the product was purified by flash chromatography on silica gel to afford the desired products (petroleum ether/ ethyl acetate $=3: 1$ ).

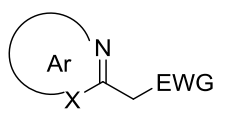

$1,0.20 \mathrm{mmol}$

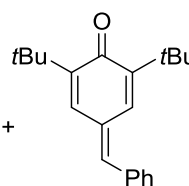

2a, $0.25 \mathrm{mmol}$
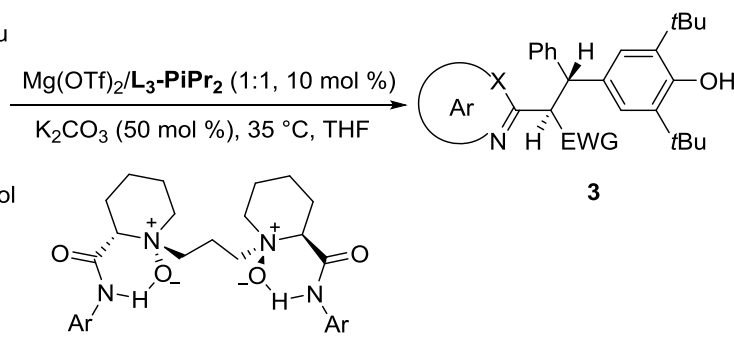

$\mathrm{L}_{3}-\mathrm{PiPr}_{2}: \mathrm{Ar}=2,6-i \mathrm{Pr}_{2} \mathrm{C}_{6} \mathrm{H}_{3}$

1 (0.20 mmol), $\mathrm{Mg}(\mathrm{OTf})_{2}(0.02 \mathrm{mmol}, 6.4 \mathrm{mg}), \mathbf{L}_{3}-\mathbf{P i P r}_{2}(0.02 \mathrm{mmol}, 13.0 \mathrm{mg})$ and $\mathrm{K}_{2} \mathrm{CO}_{3}(0.1$ $\mathrm{mmol}, 13.8 \mathrm{mg})$ were weighed into the test tube. THF $(1.0 \mathrm{~mL})$ was added, then the reaction mixture was stirred at $35{ }^{\circ} \mathrm{C}$ for 30 minutes to ensure a better coordination between the metal salt and the ligand. Then $2 \mathrm{a}(0.25 \mathrm{mmol})$ was added to the test tube. The mixture was stirred and detected by TLC (petroleum ether/ ethyl acetate $=3: 1$ ). After completion, the product was purified by flash chromatography on silica gel to afford the desired products (petroleum ether/ ethyl acetate $=3: 1$ ). 


\section{Characterization of products}

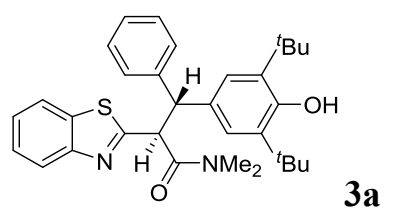

(2S,3R)-2-(benzo[d]thiazol-2-yl)-3-(3,5-di-tert-butyl-4-hydroxyphenyl)- $N, N$-dimethyl-3phenylpropanamide

$0.2 \mathrm{mmol}$ scale reaction; purified by petroleum ether/ ethyl acetate $=3: 1 ; 101.5 \mathrm{mg}$, white solid; $99 \%$ yield, $93: 7 \mathrm{dr}, 99 \% / 32 \%$ ee; determined by HPLC analysis [Daicel chiralpak ADH, $n$-hexane $/ i$ $\operatorname{PrOH}=90 / 10,1.0 \mathrm{~mL} / \mathrm{min}, \lambda=254 \mathrm{~nm}, \mathrm{t}_{1}=5.86 \mathrm{~min}, \mathrm{t}_{2}=6.98 \mathrm{~min}, \mathrm{t}_{3}=13.09 \mathrm{~min}, \mathrm{t}_{4}=31.43$ $\min ]$;

${ }^{1}$ H NMR (400 MHz, Chloroform- $d$ ) $\delta 7.87-7.85(\mathrm{~m}, 1 \mathrm{H}), 7.75-7.73(\mathrm{~m}, 1 \mathrm{H}), 7.36-7.33(\mathrm{~m}$, $3 \mathrm{H}), 7.33-7.30(\mathrm{~s}, 1 \mathrm{H}), 7.25-7.10(\mathrm{~m}, 4 \mathrm{H}), 7.04-6.92(\mathrm{~m}, 1 \mathrm{H}), 5.45-5,42(\mathrm{~m}, 1 \mathrm{H}), 5.30(\mathrm{~s}$, 0H), $5.09(\mathrm{~s}, 1 \mathrm{H}), 4.92-4.87(\mathrm{~m}, 1 \mathrm{H}), 2.91(\mathrm{~s}, 3 \mathrm{H}), 2.76(\mathrm{~s}, 3 \mathrm{H}), 1.40(\mathrm{~s}, 17 \mathrm{H}), 1.20(\mathrm{~s}, 1 \mathrm{H})$;

${ }^{13}$ C NMR (101 MHz, Chloroform- $d$ ) $\delta$ 169.9, 169.2, 152.5, 151.6, 140.9, 135.9, 135.7, 132.4, 128.6, 128.2, 126.3, 125.5, 124.7, 124.5, 122.5, 121.6, 55.7, 53.2, 34.4, 30.3;

IR (neat): 3628, 3055, 2950, 2872, 1645, 1599, 1495, 1451, 1393, 1359, 1208, 759, 729, $696 \mathrm{~cm}^{-1}$; HRMS (ESI) scaled for $[\mathrm{M}+\mathrm{H}]^{+}: \mathrm{C}_{32} \mathrm{H}_{39} \mathrm{~N}_{2} \mathrm{O}_{2} \mathrm{~S}, \mathrm{~m} / \mathrm{z}: 515.2727$, observed: 515.2728;

$[\alpha]_{365^{23}}=-123.70^{\circ}\left(c 0.81, \mathrm{CH}_{2} \mathrm{Cl}_{2}\right)$;

m.p. $=45^{\circ} \mathrm{C}$.

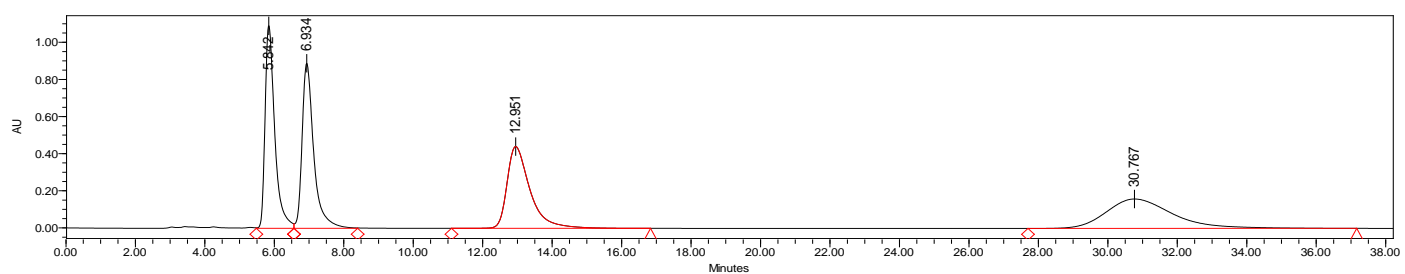

\begin{tabular}{|l|c|c|c|}
\hline & Retention Time & Area & \% Area \\
\hline 1 & 5.842 & 21068987 & 25.34 \\
\hline 2 & 6.934 & 20792521 & 25.01 \\
\hline 3 & 12.951 & 20240303 & 24.35 \\
\hline 4 & 30.767 & 21034311 & 25.30 \\
\hline
\end{tabular}

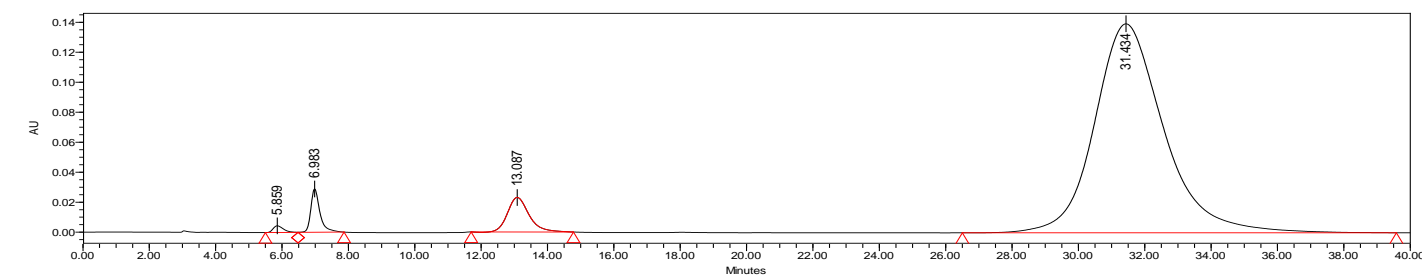

\begin{tabular}{|l|c|c|c|}
\hline & Retention Time & Area & \% Area \\
\hline 1 & 5.859 & 95781 & 0.44 \\
\hline 2 & 6.983 & 551407 & 2.52 \\
\hline 3 & 13.087 & 1044505 & 4.77 \\
\hline 4 & 31.434 & 20208565 & 92.28 \\
\hline
\end{tabular}




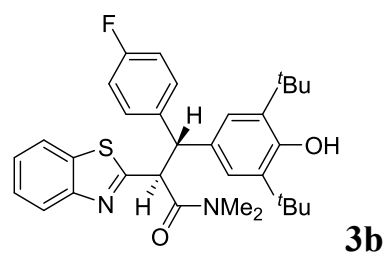

(2S,3R)-2-(benzo[d] thiazol-2-yl)-3-(3,5-di-tert-butyl-4-hydroxyphenyl)-3-(4-fluorophenyl)$\mathrm{N}, \mathrm{N}$-dimethylpropanamide

$0.2 \mathrm{mmol}$ scale reaction; purified by petroleum ether/ ethyl acetate $=3: 1 ; 106.2 \mathrm{mg}$, white solid; $99 \%$ yield, 91:9 dr, 99\%/21\% ee; determined by HPLC analysis [Daicel chiralpak ADH, $n$-hexane $/ i$ $\mathrm{PrOH}=90 / 10,1.0 \mathrm{~mL} / \mathrm{min}, \lambda=254 \mathrm{~nm}, \mathrm{t}_{1}=6.13 \mathrm{~min}, \mathrm{t}_{2}=10.08 \mathrm{~min}, \mathrm{t}_{3}=11.76 \mathrm{~min}, \mathrm{t}_{4}=19.31$ $\min ]$;

${ }^{1}$ H NMR (400 MHz, Chloroform- $\left.d\right) \delta 7.88-7.86(\mathrm{~m}, 1 \mathrm{H}), 7.77-7.73(\mathrm{~m}, 1 \mathrm{H}), 7.39-7.26(\mathrm{~m}$, $4 \mathrm{H}), 7.14-6.98(\mathrm{~m}, 2 \mathrm{H}), 6.93-6.75(\mathrm{~m}, 2 \mathrm{H}), 5.39-5.36(\mathrm{~m}, 1 \mathrm{H}), 5.14(\mathrm{~s}, 1 \mathrm{H}), 4.94-4.89(\mathrm{~m}$, $1 \mathrm{H}), 2.90(\mathrm{~s}, 3 \mathrm{H}), 2.77(\mathrm{~s}, 3 \mathrm{H}), 1.41(\mathrm{~s}, 16 \mathrm{H}), 1.20(\mathrm{~s}, 2 \mathrm{H})$;

${ }^{13}$ C NMR (101 MHz, Chloroform- $\left.d\right) \delta 169.7,168.9,161.2\left(\mathrm{~d}, J_{\mathrm{C}-\mathrm{F}}=243.2 \mathrm{~Hz}, 1 \mathrm{C}\right), 152.6,151.5$, $136.6\left(\mathrm{~d}, J_{\mathrm{C}-\mathrm{F}}=3.2 \mathrm{~Hz}, 1 \mathrm{C}\right), 135.8,135.7,132.2,130.0\left(\mathrm{~d}, J_{\mathrm{C}-\mathrm{F}}=7.9 \mathrm{~Hz}, 1 \mathrm{C}\right), 125.6,124.8,124.3$, $122.5,121.5,115.1\left(\mathrm{~d}, J_{\mathrm{C}-\mathrm{F}}=20.0 \mathrm{~Hz}, 1 \mathrm{C}\right), 55.0,53.6,37.5,35.9,34.3,30.3$;

${ }^{19}$ F NMR (376 MHz, Chloroform- $d$ ) $\delta$-116.4;

IR (neat): $3624 \mathrm{~cm}^{-1}, 3055,2950,1645,1558,1504,1453,1394,1360,1220,762,731,674 \mathrm{~cm}^{-1}$; HRMS (ESI) scaled for $[\mathrm{M}+\mathrm{H}]^{+}: \mathrm{C}_{32} \mathrm{H}_{38} \mathrm{FN}_{2} \mathrm{O}_{2} \mathrm{~S}, \mathrm{~m} / \mathrm{z}$ : 533.2633, observed: 533.2632;

$[\alpha]_{365^{23}}=-150.3^{\circ}\left(c 2.00, \mathrm{CH}_{2} \mathrm{Cl}_{2}\right)$;

m.p. $=61{ }^{\circ} \mathrm{C}$.

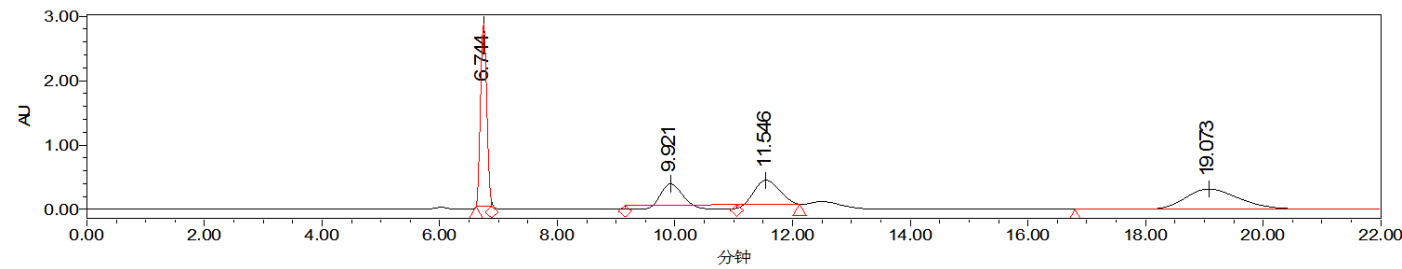

\begin{tabular}{|l|c|c|c|}
\hline & Retention Time & Area & $\%$ Area \\
\hline 1 & 6.744 & 20950682 & 32.78 \\
\hline 2 & 9.921 & 11478517 & 17.96 \\
\hline 3 & 11.546 & 11094408 & 17.36 \\
\hline 4 & 19.073 & 20391947 & 31.90 \\
\hline
\end{tabular}

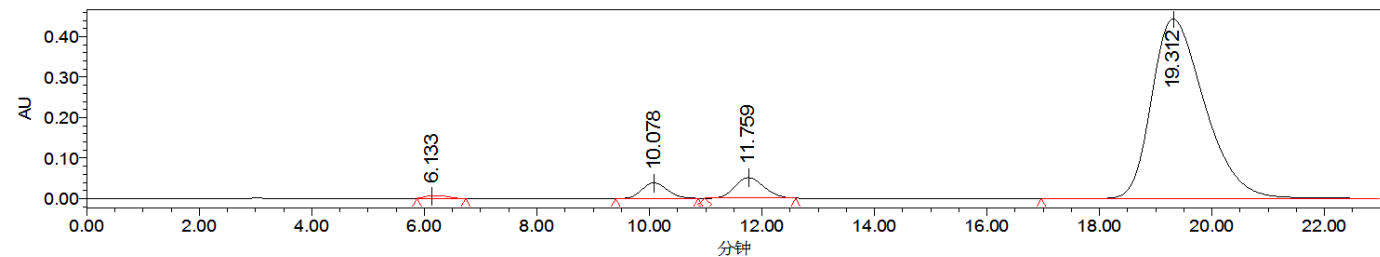

\begin{tabular}{|l|c|c|c|}
\hline & Retention Time & Area & \% Area \\
\hline 1 & 6.133 & 177048 & 0.55 \\
\hline 2 & 10.078 & 1207776 & 3.75 \\
\hline 3 & 11.759 & 1824474 & 5.67 \\
\hline 4 & 19.312 & 28962645 & 90.02 \\
\hline
\end{tabular}




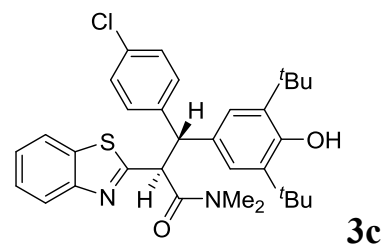

(2S,3R)-2-(benzo[d]thiazol-2-yl)-3-(4-chlorophenyl)-3-(3,5-di-tert-butyl-4-hydroxyphenyl)$\mathrm{N}, \mathbf{N}$-dimethylpropanamide

$0.2 \mathrm{mmol}$ scale reaction; purified by petroleum ether/ ethyl acetate $=3: 1 ; 107.0 \mathrm{mg}$, white solid; $98 \%$ yield, $88: 12 \mathrm{dr}$, 99\%/22\% ee; determined by HPLC analysis [Daicel chiralpak ADH, $n$-hexane/i$\mathrm{PrOH}=95 / 5,1.0 \mathrm{~mL} / \mathrm{min}, \lambda=254 \mathrm{~nm}, \mathrm{t}_{1}=12.88 \mathrm{~min}, \mathrm{t}_{2}=24.48 \mathrm{~min}, \mathrm{t}_{3}=28.18 \mathrm{~min}, \mathrm{t}_{4}=52.62$ $\min ]$;

${ }^{1}$ H NMR (400 MHz, Chloroform- $d$ ) $\delta 7.88-7.85(\mathrm{~m}, 1 \mathrm{H}), 7.76-7.73(\mathrm{~m}, 1 \mathrm{H}), 7.41-7.20(\mathrm{~m}$, 5H), $7.15-7.06(\mathrm{~m}, 3 \mathrm{H}), 5.40-5.37(\mathrm{~m}, 1 \mathrm{H}), 5.20-4.98(\mathrm{~m}, 1 \mathrm{H}), 4.97-4.88(\mathrm{~m}, 1 \mathrm{H}), 2.91(\mathrm{~s}$, $3 \mathrm{H}), 2.79-2.75(\mathrm{~m}, 3 \mathrm{H}), 1.40(\mathrm{~s}, 16 \mathrm{H}), 1.20(\mathrm{~s}, 2 \mathrm{H})$;

${ }^{13}$ C NMR (101 MHz, Chloroform- $d$ ) $\delta$ 169.6, 168.7, 152.6, 151.5, 139.5, 135.8, 135.7, 131.9, 131.9, $129.9,128.3,125.6,124.8,124.3,122.6,121.5,55.1,53.0,37.3,35.9,34.3,30.2$;

IR (neat): 3626, 3066, 2953, 1644, 1590, 1488, 1453, 1396, 1360, 1209, 759, 729, $703 \mathrm{~cm}^{-1}$;

HRMS (ESI) scaled for $[\mathrm{M}+\mathrm{H}]^{+}: \mathrm{C}_{32} \mathrm{H}_{38} \mathrm{ClN}_{2} \mathrm{O}_{2} \mathrm{~S}, \mathrm{~m} / \mathrm{z}: 549.2337,551.2307$, observed: 549.2339, 551.2314;

$[\alpha]_{365}{ }^{23}=-150.4^{\circ}\left(c 1.12, \mathrm{CH}_{2} \mathrm{Cl}_{2}\right)$;

m.p. $=58{ }^{\circ} \mathrm{C}$.

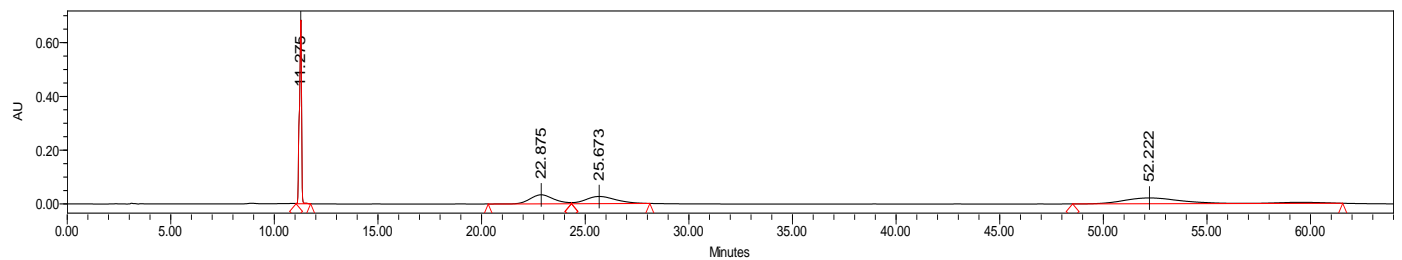

\begin{tabular}{|l|c|c|c|}
\hline & Retention Time & Area & \% Area \\
\hline 1 & 11.275 & 4849791 & 32.56 \\
\hline 2 & 22.875 & 2648438 & 17.78 \\
\hline 3 & 25.673 & 2700487 & 18.13 \\
\hline 4 & 52.222 & 4696265 & 31.53 \\
\hline
\end{tabular}

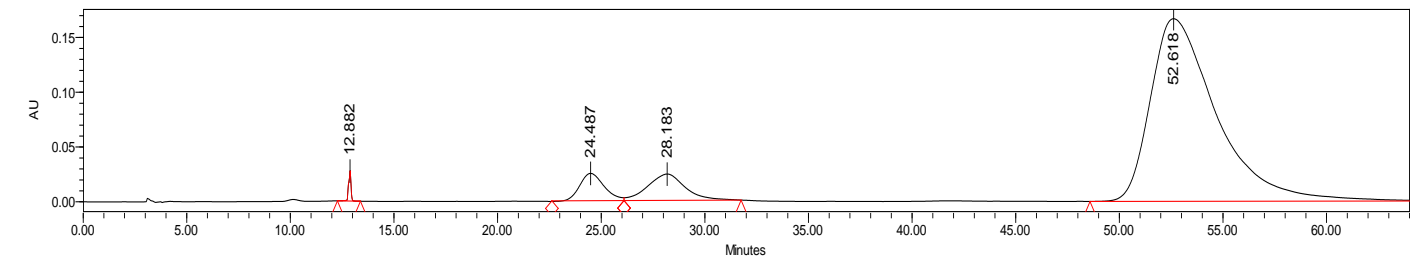

\begin{tabular}{|c|c|c|c|}
\hline & Retention Time & Area & \% Area \\
\hline 1 & 12.882 & 219989 & 0.52 \\
\hline 2 & 24.487 & 2041478 & 4.85 \\
\hline 3 & 28.183 & 3014574 & 7.16 \\
\hline 4 & 52.618 & 36825777 & 87.47 \\
\hline
\end{tabular}




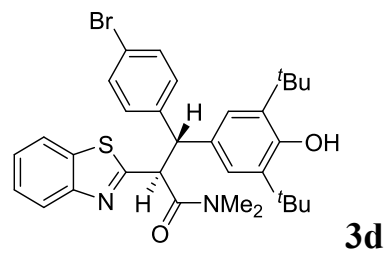

(2S,3R)-2-(benzo[d]thiazol-2-yl)-3-(4-bromophenyl)-3-(3,5-di-tert-butyl-4-hydroxyphenyl)$\mathrm{N}, \mathrm{N}$-dimethylpropanamide

$0.2 \mathrm{mmol}$ scale reaction; purified by petroleum ether/ ethyl acetate $=3: 1 ; 118.3 \mathrm{mg}$, white solid; 99.9\% yield, $86: 14 \mathrm{dr}, 99 \% / 17 \%$ ee; determined by HPLC analysis [Daicel chiralpak IA, $n$-hexane $/ i$ $\mathrm{PrOH}=90 / 10,1.0 \mathrm{~mL} / \mathrm{min}, \lambda=254 \mathrm{~nm}, \mathrm{t}_{1}=6.98 \mathrm{~min}, \mathrm{t}_{2}=10.52 \mathrm{~min}, \mathrm{t}_{3}=12.06 \mathrm{~min}, \mathrm{t}_{2}=14.62$ $\min ]$;

${ }^{1}$ H NMR (400 MHz, Chloroform- $\left.d\right) \delta 7.88-7,85(\mathrm{~m}, 1 \mathrm{H}), 7.76-7.73(\mathrm{~m}, 1 \mathrm{H}), 7.44-7.33(\mathrm{~m}$, 1H), $7.28-7.26(\mathrm{~m}, 1 \mathrm{H}), 7.24(\mathrm{~s}, 3 \mathrm{H}), 7.12-6.90(\mathrm{~m}, 2 \mathrm{H}), 5.40-5.37(\mathrm{~m}, 1 \mathrm{H}), 5.15(\mathrm{~s}, 1 \mathrm{H}), 4.90$ $-4.87(\mathrm{~m}, 1 \mathrm{H}), 2.91(\mathrm{~s}, 3 \mathrm{H}), 2.75(\mathrm{~s}, 3 \mathrm{H}), 1.40(\mathrm{~s}, 16 \mathrm{H}), 1.20(\mathrm{~s}, 2 \mathrm{H})$;

${ }^{13}$ C NMR (101 MHz, Chloroform- $d$ ) $\delta$ 169.5, 168.6, 152.6, 151.5, 140.0, 135.8, 135.7, 131.8, 131.2, 130.2, 125.6, 124.8, 124.2, 122.5, 121.5, 120.1, 55.1, 52.9, 37.3, 35.9, 34.3, 30.2;

IR (neat): 3630, 3070, 2952, 2871, 1644, 1588, 1485, 14513, 1396, 1361, 1210, 759, 731, $691 \mathrm{~cm}^{-}$ 1 ;

HRMS (ESI) scaled for $[\mathrm{M}+\mathrm{Na}]^{+}: \mathrm{C}_{32} \mathrm{H}_{37} \mathrm{BrN}_{2} \mathrm{O}_{2} \mathrm{SNa}, \mathrm{m} / \mathrm{z}: 615.1651,617.1631$, observed: 615.1654, 617.1638;

$[\boldsymbol{\alpha}]_{\mathbf{3 6 5}} \mathbf{2 3}^{23}-69.4^{\circ}\left(c\right.$ 2.44, $\left.\mathrm{CH}_{2} \mathrm{Cl}_{2}\right)$;

m.p. $=66^{\circ} \mathrm{C}$.

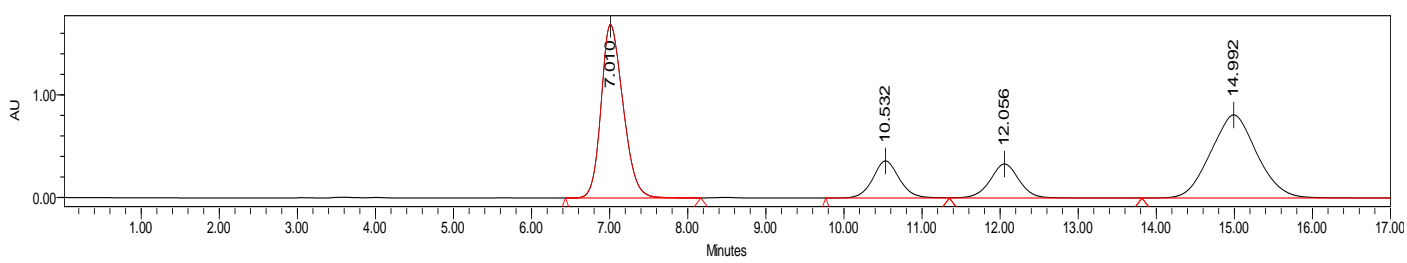

\begin{tabular}{|l|c|c|c|}
\hline & Retention Time & Area & \% Area \\
\hline 1 & 7.010 & 33067891 & 39.62 \\
\hline 2 & 10.532 & 8535375 & 10.23 \\
\hline 3 & 12.056 & 8553133 & 10.25 \\
\hline 4 & 14.992 & 33304418 & 39.90 \\
\hline
\end{tabular}

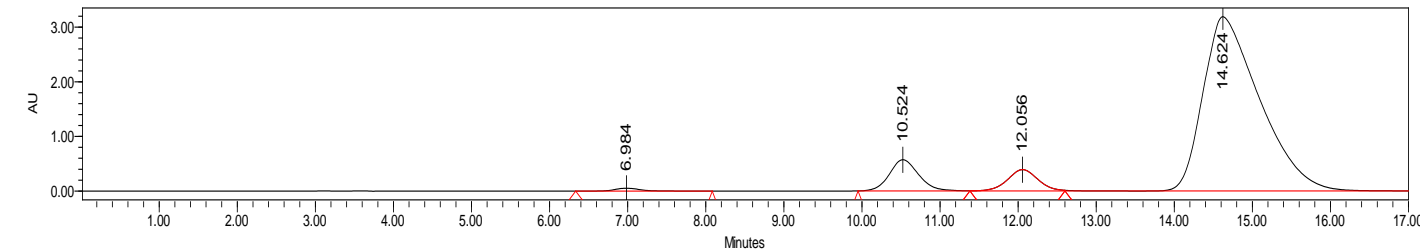

\begin{tabular}{|l|c|c|c|}
\hline & Retention Time & Area & \% Area \\
\hline 1 & 6.984 & 1160872 & 0.62 \\
\hline 2 & 10.524 & 14870887 & 7.94 \\
\hline 3 & 12.056 & 10554745 & 5.63 \\
\hline 4 & 14.624 & 160739130 & 85.81 \\
\hline
\end{tabular}




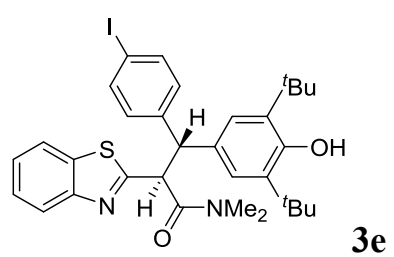

(2S,3R)-2-(benzo[d] thiazol-2-yl)-3-(3,5-di-tert-butyl-4-hydroxyphenyl)-3-(4-iodophenyl)- $N, N$ dimethylpropanamide

$0.2 \mathrm{mmol}$ scale reaction; purified by petroleum ether/ ethyl acetate $=3: 1 ; 127.5 \mathrm{mg}$, white solid; $99 \%$ yield, 85:15 dr, 98\%/25\% ee; determined by HPLC analysis [Daicel chiralpak ADH, $n$-hexane $/ i$ $\mathrm{PrOH}=95 / 5,1.0 \mathrm{~mL} / \mathrm{min}, \lambda=254 \mathrm{~nm}, \mathrm{t}_{1}=9.91 \mathrm{~min}, \mathrm{t}_{2}=28.07 \mathrm{~min}, \mathrm{t}_{3}=30.31 \mathrm{~min}, \mathrm{t}_{4}=74.66$ $\min ]$;

${ }^{1}$ H NMR (400 MHz, Chloroform- $d$ ) $\delta 7.89-7.85(\mathrm{~m}, 1 \mathrm{H}), 7.76-7.73(\mathrm{~m}, 1 \mathrm{H}), 7.64-7.43(\mathrm{~m}, 2 \mathrm{H})$, $7.39-7.33(\mathrm{~m}, 1 \mathrm{H}), 7.29-7.25(\mathrm{~m}, 1 \mathrm{H}), 7.18-6.87(\mathrm{~m}, 4 \mathrm{H}), 5.40-5.37(\mathrm{~m}, 1 \mathrm{H}), 5.21-5.14(\mathrm{~m}$, $1 \mathrm{H}), 4.93-4.82(\mathrm{~m}, 1 \mathrm{H}), 2.91(\mathrm{~s}, 3 \mathrm{H}), 2.75(\mathrm{~s}, 3 \mathrm{H}), 1.40(\mathrm{~s}, 16 \mathrm{H}), 1.20(\mathrm{~s}, 2 \mathrm{H})$;

${ }^{13}$ C NMR (101 MHz, Chloroform- $d$ ) $\delta$ 169.5, 168.6, 152.6, 151.5, 140.7 , 137.2, 135.8, 135.7, 131.8, $130.5,125.6,124.8,124.2,122.5,121.5,91.9,55.2,52.8,37.3,35.9,34.3,30.2$;

IR (neat): $3626 \mathrm{~cm}^{-1}, 2951,2870,1644,1559,1501,1454,1395,1361,1208,757,728,687 \mathrm{~cm}^{-1}$; HRMS (ESI) scaled for $[\mathrm{M}+\mathrm{H}]^{+}: \mathrm{C}_{32} \mathrm{H}_{38} \mathrm{IN}_{2} \mathrm{O}_{2} \mathrm{~S}, \mathrm{~m} / \mathrm{z}: 641.1693$, observed: 641.1697; $[\alpha]_{365}{ }^{23}=-123.7^{\circ}\left(c 0.81, \mathrm{CH}_{2} \mathrm{Cl}_{2}\right)$; m.p. $=51{ }^{\circ} \mathrm{C}$.

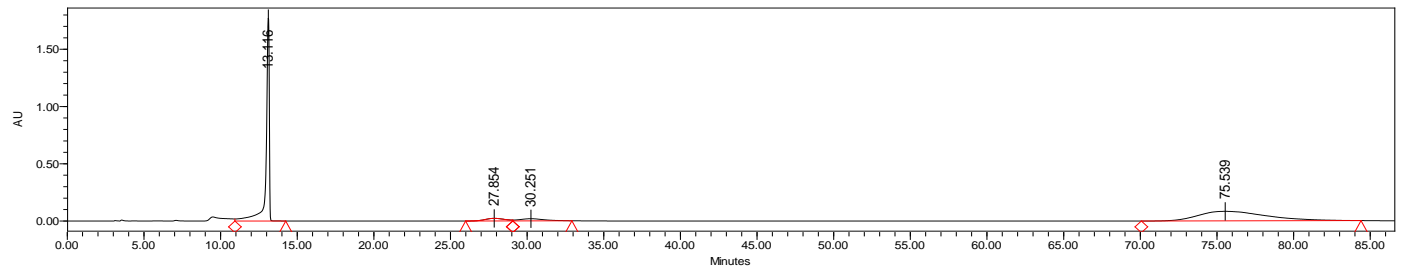

\begin{tabular}{|l|c|c|c|}
\hline & Retention Time & Area & $\%$ Area \\
\hline 1 & 13.116 & 25797153 & 46.25 \\
\hline 2 & 27.854 & 2034241 & 3.65 \\
\hline 3 & 30.251 & 2118340 & 3.80 \\
\hline 4 & 75.539 & 25830173 & 46.31 \\
\hline
\end{tabular}

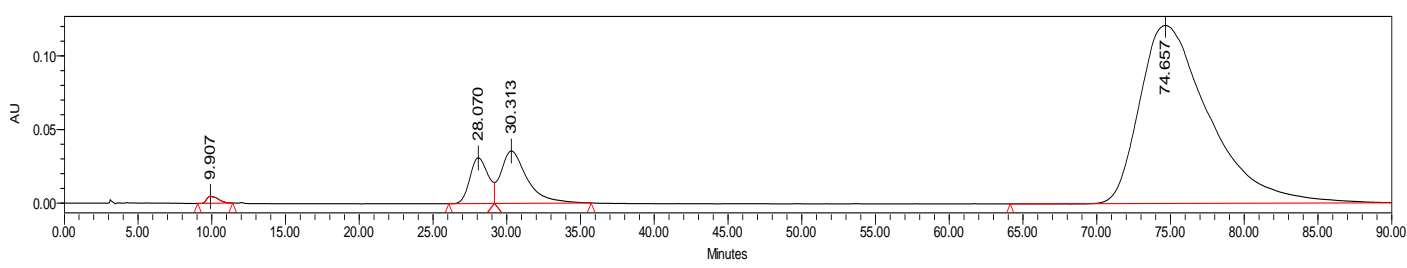

\begin{tabular}{|l|c|c|c|}
\hline & Retention Time & Area & \% Area \\
\hline 1 & 9.907 & 254817 & 0.54 \\
\hline 2 & 28.070 & 2693255 & 5.73 \\
\hline 3 & 30.313 & 4408416 & 9.38 \\
\hline 4 & 74.657 & 39665653 & 84.36 \\
\hline
\end{tabular}




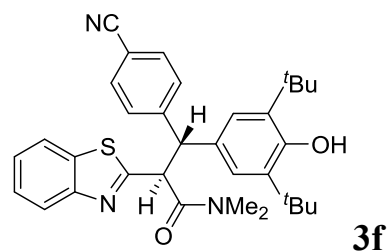

(2S,3R)-2-(benzo[d]thiazol-2-yl)-3-(4-cyanophenyl)-3-(3,5-di-tert-butyl-4-hydroxyphenyl)$\mathrm{N}, \mathrm{N}$-dimethylpropanamide

$0.2 \mathrm{mmol}$ scale reaction; purified by petroleum ether/ ethyl acetate $=3: 1 ; 104.0 \mathrm{mg}$, white solid; $96 \%$ yield, $96: 4 \mathrm{dr}$, $98 \% / 36 \%$ ee; determined by HPLC analysis [Daicel chiralpak ADH, $n$-hexane $/ i$ $\mathrm{PrOH}=80 / 20,1.0 \mathrm{~mL} / \mathrm{min}, \lambda=254 \mathrm{~nm}, \mathrm{t}_{1}=6.23 \mathrm{~min}, \mathrm{t}_{2}=10.14 \mathrm{~min}, \mathrm{t}_{3}=12.88 \mathrm{~min}, \mathrm{t}_{4}=20.46$ $\min ]$;

${ }^{1}$ H NMR (400 MHz, Chloroform- $d$ ) $\delta 7.86-7.84(\mathrm{~m}, 1 \mathrm{H}), 7.77-7.73(\mathrm{~m}, 1 \mathrm{H}), 7.55-7.33(\mathrm{~m}$, $5 \mathrm{H}), 7.32-7.26(\mathrm{~m}, 1 \mathrm{H}), 7.09-6.85(\mathrm{~m}, 2 \mathrm{H}), 5.42-5.20(\mathrm{~m}, 1 \mathrm{H}), 5.18(\mathrm{~s}, 1 \mathrm{H}), 4.96-4.95(\mathrm{~m}$, $1 \mathrm{H}), 2.94(\mathrm{~s}, 3 \mathrm{H}), 2.77$ (s, 3H), 1.39 (s, 17H), $1.18(\mathrm{~s}, 1 \mathrm{H})$;

${ }^{13}$ C NMR (101 MHz, Chloroform- $d$ ) $\delta 169.2,168.0,152.8,151.5,146.5,136.1,135.5,132.0,130.9$, 129.3, 125.8, 125.0, 124.2, 122.6, 121.5, 118.6, 110.1, 55.6, 52.7, 37.4, 36.0, 34.3, 30.2;

IR (neat): $3622 \mathrm{~cm}^{-1}, 2951,1645,1559,1480,1454,1393,1361,1212,758,730,696 \mathrm{~cm}^{-1}$;

HRMS (ESI) scaled for $[\mathrm{M}+\mathrm{H}]^{+}: \mathrm{C}_{33} \mathrm{H}_{38} \mathrm{~N}_{3} \mathrm{O}_{2} \mathrm{~S}, \mathrm{~m} / \mathrm{z}$ : 504.2679, observed: 504.2678;

$[\alpha]_{365^{23}}=-283.8^{\circ}\left(c 0.85, \mathrm{CH}_{2} \mathrm{Cl}_{2}\right)$;

m.p. $=55^{\circ} \mathrm{C}$.

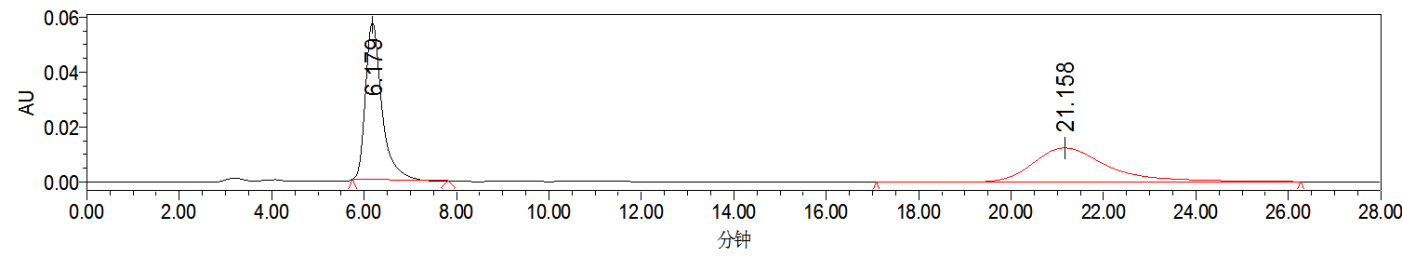

\begin{tabular}{|l|c|c|c|}
\hline & Retention Time & Area & \% Area \\
\hline 1 & 6.179 & 1412844 & 50.48 \\
\hline 2 & 21.158 & 1386043 & 49.52 \\
\hline
\end{tabular}

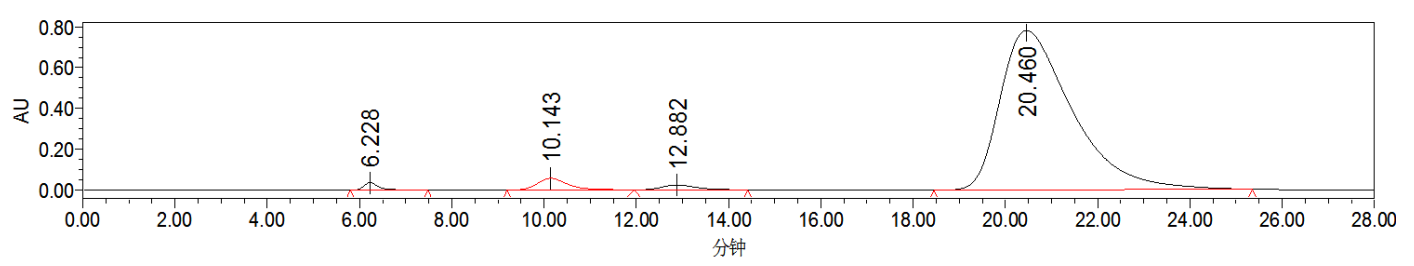

\begin{tabular}{|l|c|c|c|}
\hline & Retention Time & Area & $\%$ Area \\
\hline 1 & 6.228 & 803627 & 0.90 \\
\hline 2 & 10.143 & 2640770 & 2.96 \\
\hline 3 & 12.882 & 1249270 & 1.40 \\
\hline 4 & 20.460 & 84526660 & 94.74 \\
\hline
\end{tabular}




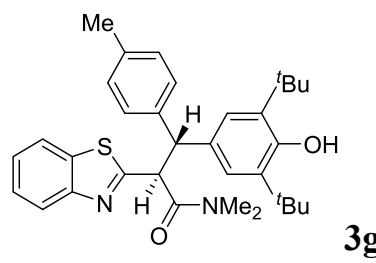

(2S,3R)-2-(benzo[d]thiazol-2-yl)-3-(3,5-di-tert-butyl-4-hydroxyphenyl)- $N, N$-dimethyl-3-(ptolyl)propenamide

$0.2 \mathrm{mmol}$ scale reaction; purified by petroleum ether/ ethyl acetate $=3: 1 ; 105 \mathrm{mg}$, white solid; $99 \%$ yield, $85: 15 \mathrm{dr}, 98 \% / 45 \%$ ee; determined by HPLC analysis [Daicel chiralpak ADH, $n$-hexane $/ i$ $\mathrm{PrOH}=90 / 10,1.0 \mathrm{~mL} / \mathrm{min}, \lambda=254 \mathrm{~nm}, \mathrm{t}_{1}=5.67 \mathrm{~min}, \mathrm{t}_{2}=6.65 \mathrm{~min}, \mathrm{t}_{3}=12.72 \mathrm{~min}, \mathrm{t}_{4}=29.90$ $\min ]$;

${ }^{1}$ H NMR (400 MHz, Chloroform- $d$ ) $\delta 7.88-7.83(\mathrm{~m}, 1 \mathrm{H}), 7.79-7.72(\mathrm{~m}, 1 \mathrm{H}), 7.41-7.32(\mathrm{~m}$, $1 \mathrm{H}), 7.31-7.26(\mathrm{~m}, 1 \mathrm{H}), 7.26-7.21(\mathrm{~m}, 2 \mathrm{H}), 7.16-7.09(\mathrm{~m}, 2 \mathrm{H}), 6.93-6.92(\mathrm{~m}, 2 \mathrm{H}), 5.44-4.01$ (m, 1H), $5.08(\mathrm{~s}, 1 \mathrm{H}), 4.92-4.82(\mathrm{~m}, 1 \mathrm{H}), 2.90(\mathrm{~s}, 3 \mathrm{H}), 2.74(\mathrm{~s}, 3 \mathrm{H}), 2.14(\mathrm{~s}, 3 \mathrm{H}), 1.40(\mathrm{~s}, 15 \mathrm{H})$, $1.20(\mathrm{~s}, 3 \mathrm{H})$;

${ }^{13}$ C NMR (101 MHz, Chloroform- $d$ ) $\delta 170.0,169.5,152.5,151.6,137.8,135.9,135.7,135.6,132.6$, 128.9, 128.3, 125.4, 124.6, 124.4, 122.5, 121.6, 55.4, 53.2, 37.4, 36.0, 34.3, 30.3, 20.9;

IR (neat): $3631 \mathrm{~cm}^{-1}, 3053,2953,2871,1644,1557,1483,1452,1394,1360,1210,759,672 \mathrm{~cm}^{-1}$; HRMS (ESI) scaled for $[\mathrm{M}+\mathrm{H}]^{+}: \mathrm{C}_{33} \mathrm{H}_{41} \mathrm{~N}_{2} \mathrm{O}_{2} \mathrm{~S}, \mathrm{~m} / \mathrm{z}$ : 529.2883, observed: 529.2883; $[\alpha]_{365^{23}}=-40.1^{\circ}\left(c 0.67, \mathrm{CH}_{2} \mathrm{Cl}_{2}\right)$; m.p. $=70^{\circ} \mathrm{C}$.

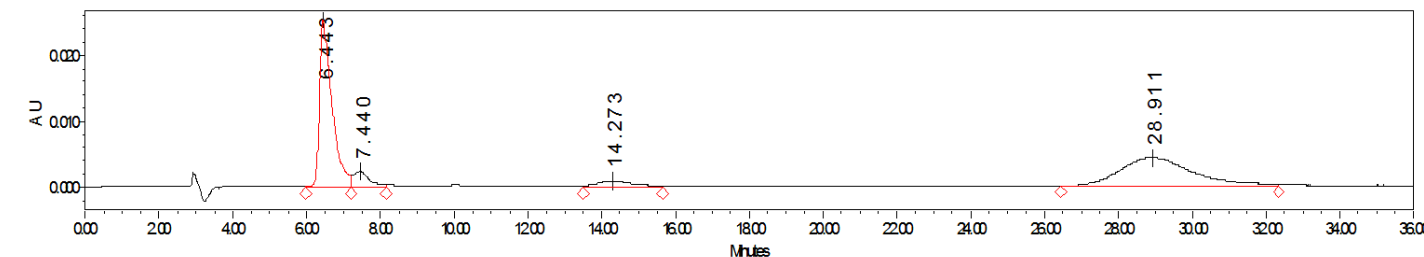

\begin{tabular}{|l|c|c|c|}
\hline & Retention Time & Area & \% Area \\
\hline 1 & 6.443 & 609882 & 45.65 \\
\hline 2 & 7.440 & 78286 & 5.86 \\
\hline 3 & 14.273 & 65780 & 4.92 \\
\hline 4 & 28.911 & 582100 & 43.57 \\
\hline
\end{tabular}

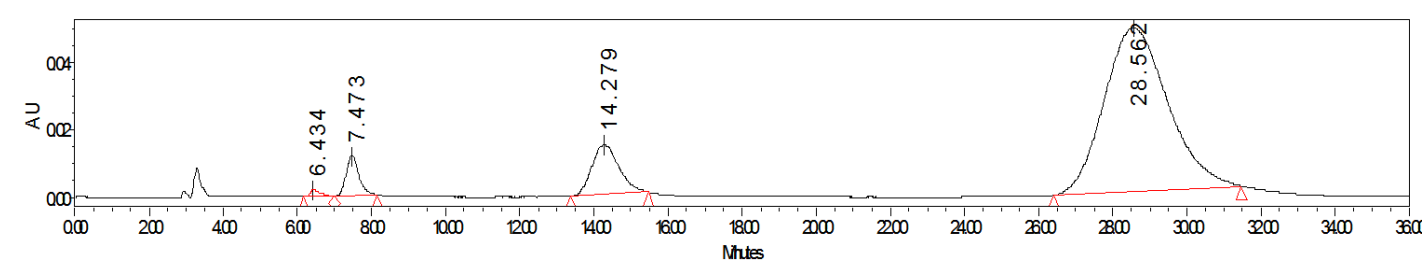

\begin{tabular}{|l|c|c|c|}
\hline & Retention Time & Area & \% Area \\
\hline 1 & 6.434 & 39531 & 0.57 \\
\hline 2 & 7.473 & 284942 & 4.14 \\
\hline 3 & 14.279 & 756086 & 10.99 \\
\hline 4 & 28.562 & 5797320 & 84.29 \\
\hline
\end{tabular}




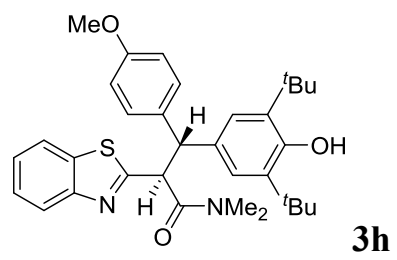

(2S,3R)-2-(benzo[d]thiazol-2-yl)-3-(3,5-di-tert-butyl-4-hydroxyphenyl)-3-(4-methoxyphenyl)$\mathrm{N}, \mathrm{N}$-dimethylpropanamide

$0.2 \mathrm{mmol}$ scale reaction; purified by petroleum ether/ ethyl acetate $=3: 1 ; 108.6 \mathrm{mg}$, white solid; $99 \%$ yield, $>19: 1 \mathrm{dr}$, 99\% ee; determined by HPLC analysis [Daicel chiralpak ADH, $n$-hexane $/ i-\mathrm{PrOH}=$ $\left.90 / 10,1.0 \mathrm{~mL} / \mathrm{min}, \lambda=254 \mathrm{~nm}, \mathrm{t}_{1}=6.81 \mathrm{~min}, \mathrm{t}_{4}=46.08 \mathrm{~min}\right]$;

${ }^{1}$ H NMR $(400 \mathrm{MHz}$, Chloroform- $d$ ) $\delta 7.86(\mathrm{~d}, J=8.1 \mathrm{~Hz}, 1 \mathrm{H}), 7.72(\mathrm{~d}, J=8.0 \mathrm{~Hz}, 1 \mathrm{H}), 7.36-7.24$ (m, 4H), $7.16(\mathrm{~s}, 2 \mathrm{H}), 6.67-6.65(\mathrm{~m}, 2 \mathrm{H}), 5.40-5.37(\mathrm{~m}, 1 \mathrm{H}), 5.12(\mathrm{~s}, 1 \mathrm{H}), 4.87-4.84(\mathrm{~m}, 1 \mathrm{H})$, $3.60(\mathrm{~s}, 3 \mathrm{H}), 2.89$ (s, 3H), 2.75 (s, 3H), 1.40 (s, 18H);

${ }^{13}$ C NMR (101 MHz, Chloroform- $d$ ) $\delta 169.9,169.3,157.7,152.4,151.5,135.8,135.6,133.0,132.7$, 129.5, 125.4, 124.6, 124.3, 122.4, 121.5, 113.5, 54.9, 54.8, 53.4, 37.3, 35.9, 34.3, 30.3;

IR (neat): $3621 \mathrm{~cm}^{-1}, 3067,2955,2870,1645,1599,1495,1454,1393,1361,1208,762,731,670$ $\mathrm{cm}^{-1}$;

HRMS (ESI) scaled for $[\mathrm{M}+\mathrm{H}]^{+}: \mathrm{C}_{33} \mathrm{H}_{41} \mathrm{~N}_{2} \mathrm{O}_{3} \mathrm{~S}, \mathrm{~m} / \mathrm{z}$ : 545.2832, observed: 545.2831;

$[\alpha]_{365}{ }^{23}=-53.1^{\circ}\left(c 0.99, \mathrm{CH}_{2} \mathrm{Cl}_{2}\right)$;

m.p. $=50{ }^{\circ} \mathrm{C}$.

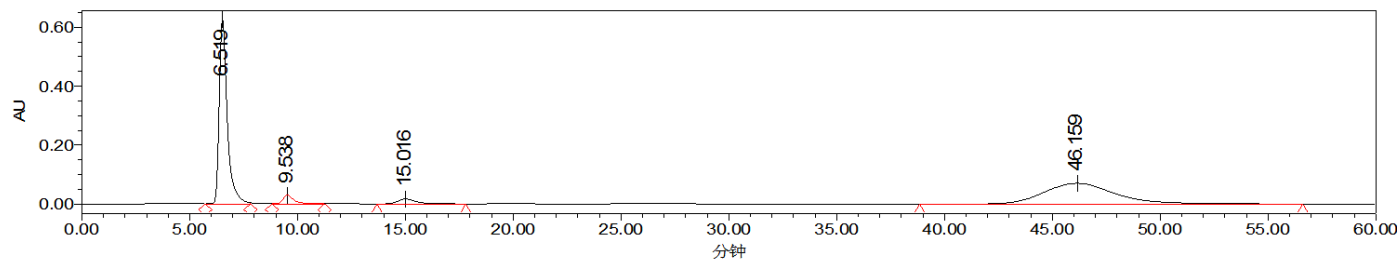

\begin{tabular}{|l|c|c|c|}
\hline & Retention Time & Area & \% Area \\
\hline 1 & 6.519 & 16332077 & 47.19 \\
\hline 2 & 9.538 & 1092691 & 3.16 \\
\hline 3 & 15.016 & 1022946 & 2.96 \\
\hline 4 & 46.159 & 16160703 & 46.70 \\
\hline
\end{tabular}

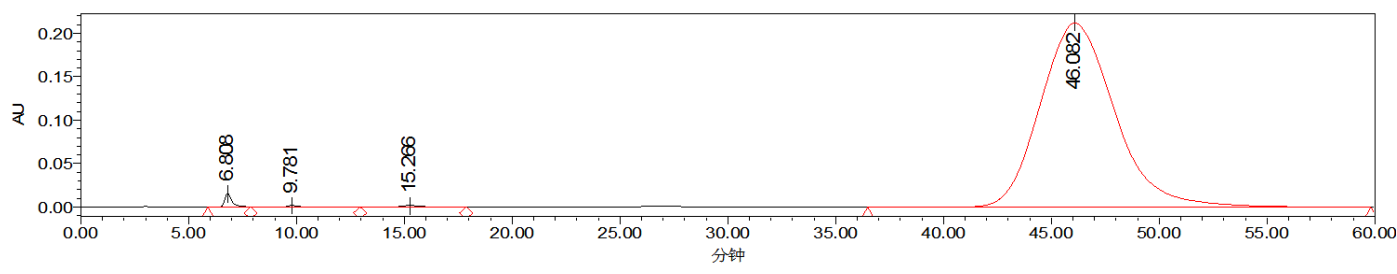

\begin{tabular}{|l|c|c|c|}
\hline & Retention Time & Area & \% Area \\
\hline 1 & 6.808 & 362045 & 0.70 \\
\hline 2 & 9.781 & 75741 & 0.15 \\
\hline 3 & 15.266 & 114086 & 0.22 \\
\hline 4 & 46.082 & 50929870 & 98.93 \\
\hline
\end{tabular}




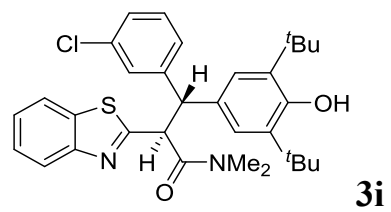

(2S,3S)-2-(benzo[d]thiazol-2-yl)-3-(3-chlorophenyl)-3-(3,5-di-tert-butyl-4-hydroxyphenyl)$\mathrm{N}, \mathrm{N}$-dimethylpropanamide

$0.2 \mathrm{mmol}$ scale reaction; purified by petroleum ether/ ethyl acetate $=3: 1 ; 106 \mathrm{mg}$, white solid; $97 \%$ yield, $84: 16 \mathrm{dr}$, 98\%/47\% ee; determined by HPLC analysis [Daicel chiralpak ADH, $n$-hexane $/ i$ $\mathrm{PrOH}=90 / 10,1.0 \mathrm{~mL} / \mathrm{min}, \lambda=254 \mathrm{~nm}, \mathrm{t}_{1}=5.63 \mathrm{~min}, \mathrm{t}_{2}=6.96 \mathrm{~min}, \mathrm{t}_{3}=10.25 \mathrm{~min}, \mathrm{t}_{4}=26.65$ $\min ]$;

${ }^{1} \mathbf{H}$ NMR (400 MHz, Chloroform- $d$ ) $\delta 7.89-7.85(\mathrm{~m}, 1 \mathrm{H}), 7.76-7.73(\mathrm{~m}, 1 \mathrm{H}), 7.40-7.32(\mathrm{~m}$, 2H), $7.32-7.25(\mathrm{~m}, 2 \mathrm{H}), 7.14(\mathrm{~s}, 2 \mathrm{H}), 7.09-6.88(\mathrm{~m}, 2 \mathrm{H}), 5.41-5.38(\mathrm{~m}, 1 \mathrm{H}), 5.17(\mathrm{~s}, 1 \mathrm{H}), 4.98$ $-4.85(\mathrm{~m}, 1 \mathrm{H}), 3.06-2.91(\mathrm{~m}, 3 \mathrm{H}), 2.80-2.75(\mathrm{~m}, 3 \mathrm{H}), 1.41-1.20(\mathrm{~m}, 18 \mathrm{H})$;

${ }^{13}$ C NMR (101 MHz, Chloroform- $d$ ) $\delta$ 169.5, 168.5, 152.6, 151.5, 142.8, 135.8, 135.6, 135.3, 133.9, 131.6, 129.3, 129.0, 126.5, 126.4, 125.6, 125.1, 124.8, 124.3, 122.5, 121.5, 55.3, 52.9, 37.3, 35.9, $34.3,30.2,30.0$;

IR (neat): 3600, 3057, 2955, 2872, 1637, 1595, 1473, 1433, 1396, 1363, 1213, 756, 727, $696 \mathrm{~cm}^{-1}$; HRMS (ESI) scaled for $[\mathrm{M}+\mathrm{H}]^{+}: \mathrm{C}_{32} \mathrm{H}_{38} \mathrm{ClN}_{2} \mathrm{O}_{2} \mathrm{~S}, \mathrm{~m} / \mathrm{z}: 549.2337,551.2313$, observed: 549.2330, 551.2307
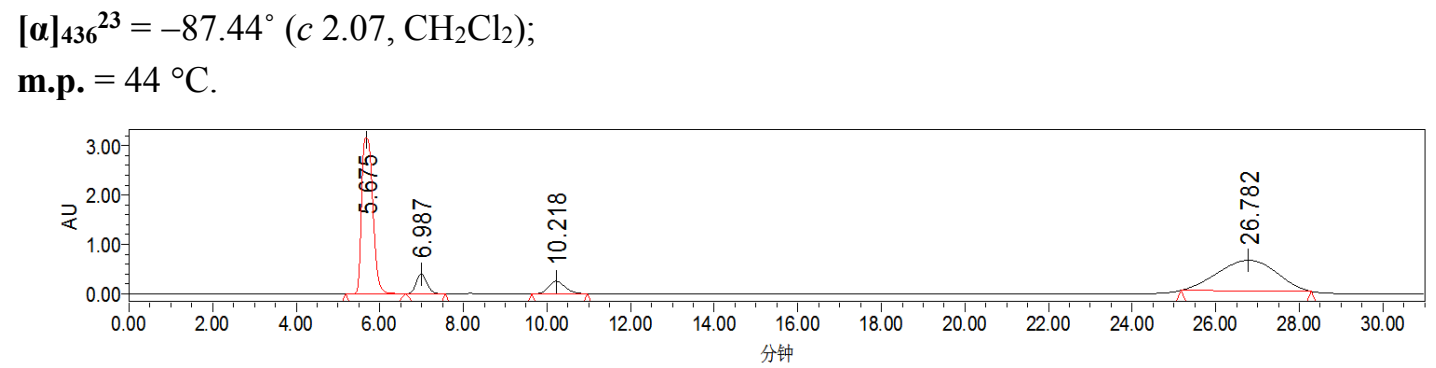

\begin{tabular}{|l|c|c|c|}
\hline & Retention Time & Area & \% Area \\
\hline 1 & 5.675 & 58505138 & 44.17 \\
\hline 2 & 6.987 & 6996170 & 5.28 \\
\hline 3 & 10.218 & 6908666 & 5.22 \\
\hline 4 & 26.782 & 60054978 & 45.34 \\
\hline
\end{tabular}

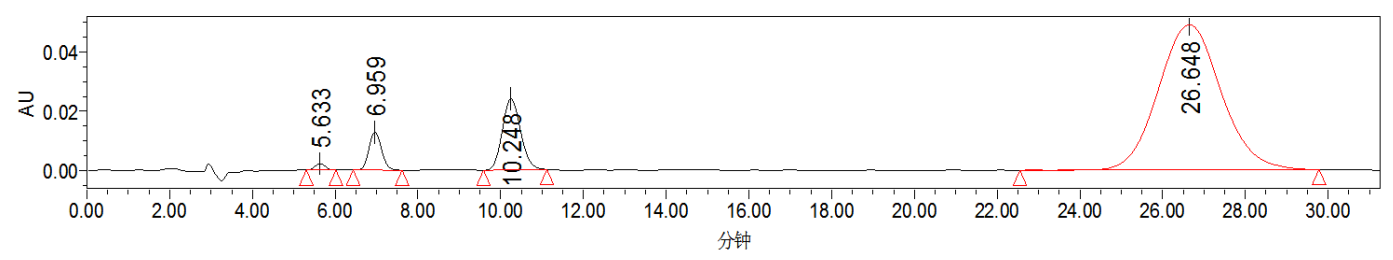

\begin{tabular}{|l|c|c|c|}
\hline & Retention Time & Area & \% Area \\
\hline 1 & 5.633 & 36606 & 0.59 \\
\hline 2 & 6.959 & 271938 & 4.39 \\
\hline 3 & 10.248 & 735482 & 11.87 \\
\hline 4 & 26.648 & 5154405 & 83.16 \\
\hline
\end{tabular}




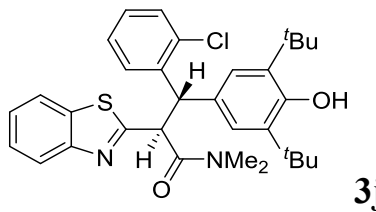

(2S,3S)-2-(benzo[d]thiazol-2-yl)-3-(2-chlorophenyl)-3-(3,5-di-tert-butyl-4-hydroxyphenyl)$\mathrm{N}, \mathrm{N}$-dimethylpropanamide

$0.2 \mathrm{mmol}$ scale reaction; purified by petroleum ether/ ethyl acetate $=3: 1 ; 108 \mathrm{mg}$, white solid; $98 \%$ yield, 90:10 dr, 99\%/96\% ee; determined by HPLC analysis [Daicel chiralpak ID, $n$-hexane $/ i$-PrOH $\left.=80 / 20,1.0 \mathrm{~mL} / \mathrm{min}, \lambda=254 \mathrm{~nm}, \mathrm{t}_{1}=5.39 \mathrm{~min}, \mathrm{t}_{2}=6.60 \mathrm{~min}, \mathrm{t}_{3}=8.59 \mathrm{~min}, \mathrm{t}_{4}=10.80 \mathrm{~min}\right]$;

${ }^{1}$ H NMR (400 MHz, Chloroform- $d$ ) $\delta 7.91-7.83(\mathrm{~m}, 1 \mathrm{H}), 7.79-7.69(\mathrm{~m}, 1 \mathrm{H}), 7.67-7.45(\mathrm{~m}$, $1 \mathrm{H}), 7.35-7.19(\mathrm{~m}, 4 \mathrm{H}), 7.18-7.15(\mathrm{~m}, 1 \mathrm{H}), 7.13-7.07(\mathrm{~m}, 1 \mathrm{H}), 6.93-6.88(\mathrm{~m}, 1 \mathrm{H}), 5.59-5.30$ (m, 2H), $5.15-4.90(\mathrm{~m}, 1 \mathrm{H}), 2.87$ (s, 3H), 2.73 (s, 3H), 1.39 (s, 16H), $1.16(\mathrm{~s}, 2 \mathrm{H})$;

${ }^{13}$ C NMR (101 MHz, Chloroform- $d$ ) $\delta 169.4,169.2,152.6,151.4,138.1,135.8,135.6,134.5,130.3$, 129.8, 128.6, 127.4, 126.3, 125.4, 124.9, 124.6, 122.3, 121.5, 52.5, 51.1, 37.3, 35.9, 34.3, 30.2;

IR (neat): 3600, 3055, 2955, 2911, 1630, 1504, 1472, 1433, 1395, 1363, 1209, 754, 727, $697 \mathrm{~cm}^{-1}$; HRMS (ESI) scaled for $[\mathrm{M}+\mathrm{H}]^{+}: \mathrm{C}_{32} \mathrm{H}_{38} \mathrm{ClN}_{2} \mathrm{O}_{2} \mathrm{~S}, \mathrm{~m} / \mathrm{z}: 549.2337$, 551.2313, observed: 549.2338, 551.2313;

$[\alpha]_{365^{23}}=160.84^{\circ}\left(c 2.20, \mathrm{CH}_{2} \mathrm{Cl}_{2}\right)$;

m.p. $=52{ }^{\circ} \mathrm{C}$.

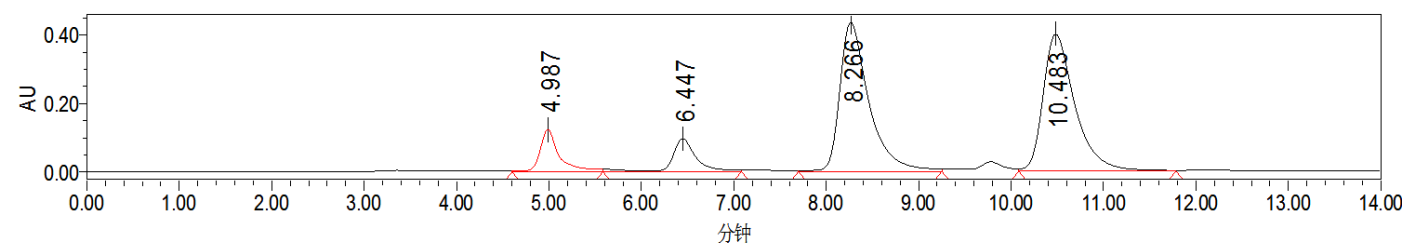

\begin{tabular}{|l|c|c|c|}
\hline & Retention Time & Area & \% Area \\
\hline 1 & 4.987 & 1819135 & 7.98 \\
\hline 2 & 6.447 & 1780239 & 7.81 \\
\hline 3 & 8.266 & 9575031 & 42.01 \\
\hline 4 & 10.483 & 9619812 & 42.20 \\
\hline
\end{tabular}

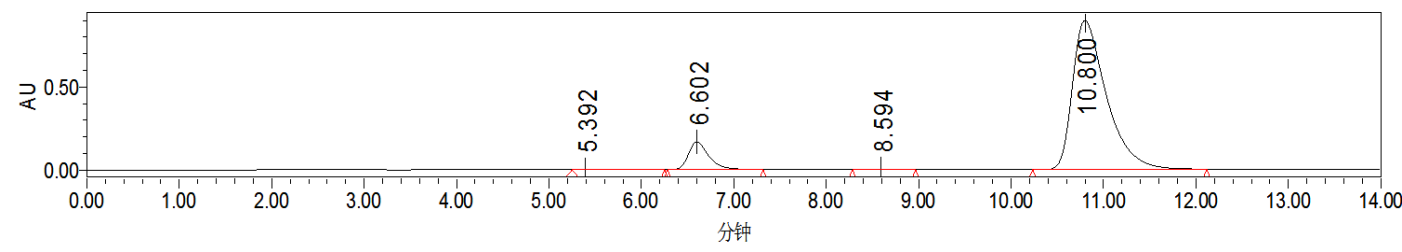

\begin{tabular}{|l|c|c|c|}
\hline & Retention Time & Area & \% Area \\
\hline 1 & 5.392 & 50754 & 0.20 \\
\hline 2 & 6.602 & 2573661 & 10.06 \\
\hline 3 & 8.594 & 58770 & 0.23 \\
\hline 4 & 10.800 & 22898351 & 89.51 \\
\hline
\end{tabular}




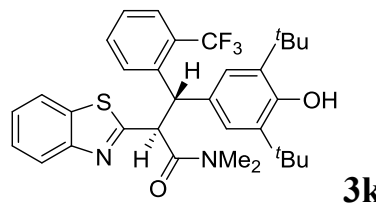

(2S,3S)-2-(benzo[d] thiazol-2-yl)-3-(3,5-di-tert-butyl-4-hydroxyphenyl)- $N, N$-dimethyl-3-(2(trifluoromethyl)phenyl)propenamide

$0.2 \mathrm{mmol}$ scale reaction; purified by petroleum ether/ ethyl acetate $=3: 1 ; 95.3 \mathrm{mg}$, white solid; $82 \%$ yield, $89: 11 \mathrm{dr}$, 99\%/93\% ee; determined by HPLC analysis [Daicel chiralpak ADH, $n$-hexane $/ i$ $\left.\mathrm{PrOH}=90 / 10,1.0 \mathrm{~mL} / \mathrm{min}, \lambda=254 \mathrm{~nm}, \mathrm{t}_{1}=3.53 \mathrm{~min}, \mathrm{t}_{2}=5.72 \mathrm{~min}, \mathrm{t}_{3}=9.68 \mathrm{~min}, \mathrm{t}_{4}=18.89 \mathrm{~min}\right]$; ${ }^{1}$ H NMR (400 MHz, Chloroform- $d$ ) $\delta 7.94-7.92(\mathrm{~m}, 1 \mathrm{H}), 7.86-7.81(\mathrm{~m}, 1 \mathrm{H}), 7.73-7.67(\mathrm{~m}$, $1 \mathrm{H}), 7.53-7.39(\mathrm{~m}, 2 \mathrm{H}), 7.38-7.30(\mathrm{~m}, 1 \mathrm{H}), 7.29-7.17(\mathrm{~m}, 3 \mathrm{H}), 7.15-6.75(\mathrm{~m}, 1 \mathrm{H}), 5.52-5.49$ (m, 1H), $5.36-5.33(\mathrm{~m}, 1 \mathrm{H}), 5.15(\mathrm{~s}, 1 \mathrm{H}), 2.76(\mathrm{~s}, 3 \mathrm{H}), 2.74(\mathrm{~s}, 3 \mathrm{H}), 1.39(\mathrm{~s}, 17 \mathrm{H}), 1.11(\mathrm{~s}, 1 \mathrm{H})$; ${ }^{13}$ C NMR (101 MHz, Chloroform- $d$ ) $\delta$ 169.7, 168.8, 152.6, 151.3, 138.7, 131.0, 130.9, 129.6, 129.0 $\left(\mathrm{d}, J_{\mathrm{C}-\mathrm{F}}=29.4 \mathrm{~Hz}, 1 \mathrm{C}\right), 126.5,126.4\left(\mathrm{~d}, J_{\mathrm{C}-\mathrm{F}}=6.1 \mathrm{~Hz}, 1 \mathrm{C}\right), 125.4,124.8,124.7,124.2\left(\mathrm{~d}, J_{\mathrm{C}-\mathrm{F}}=\right.$ 274.1 Hz, 1C), 122.3, 121.6, 53.8, 50.7, 37.2, 35.9, 34.3, 30.3;

${ }^{19}$ F NMR (376 MHz, Chloroform- $d$ ) $\delta-58.0$;

IR (neat): 3624, 3065, 2951, 2870, 1649, 1582, 1494, 1454, 1393, 1361, 1205, 769, 735, $687 \mathrm{~cm}^{-1}$; HRMS (ESI) scaled for [M+Na] $: \mathrm{C}_{33} \mathrm{H}_{37} \mathrm{~F}_{3} \mathrm{~N}_{2} \mathrm{O}_{2} \mathrm{SNa}, \mathrm{m} / \mathrm{z}: 605.2420$, observed: 605.2422; $[\boldsymbol{\alpha}]_{436^{23}}=226.2^{\circ}\left(c 1.59, \mathrm{CH}_{2} \mathrm{Cl}_{2}\right)$; m.p. $=51{ }^{\circ} \mathrm{C}$.

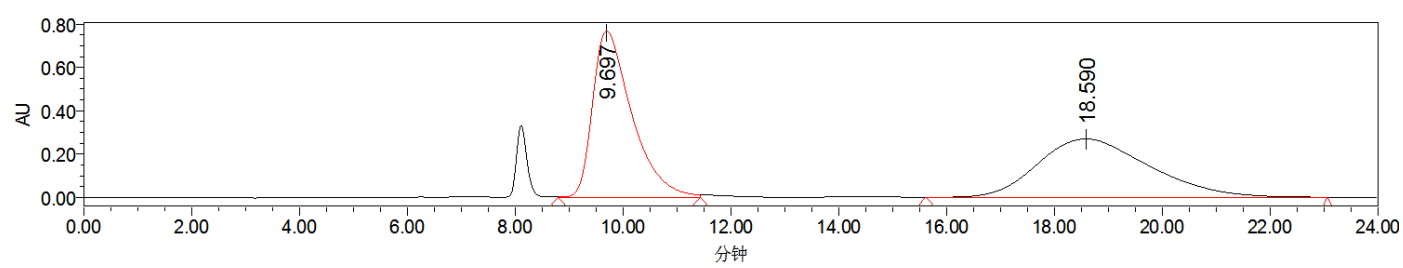

\begin{tabular}{|l|c|c|c|}
\hline & Retention Time & Area & \% Area \\
\hline 1 & 9.697 & 38709810 & 50.01 \\
\hline 2 & 18.590 & 38689821 & 49.99 \\
\hline
\end{tabular}

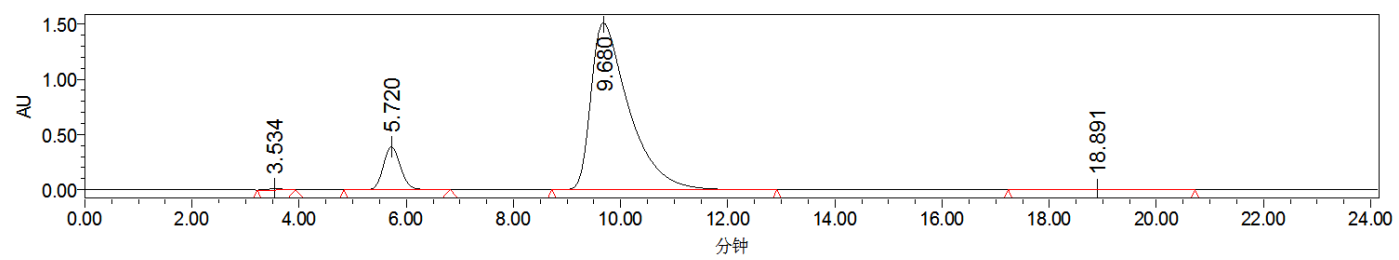

\begin{tabular}{|l|c|c|c|}
\hline & Retention Time & Area & \% Area \\
\hline 1 & 3.534 & 287443 & 0.34 \\
\hline 2 & 5.720 & 8687631 & 10.41 \\
\hline 3 & 9.680 & 74213175 & 88.94 \\
\hline 4 & 18.891 & 254919 & 0.31 \\
\hline
\end{tabular}




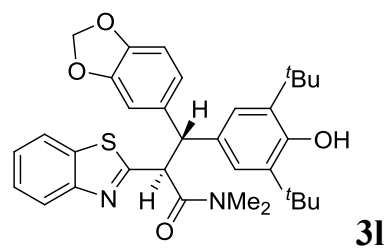

(2S,3S)-3-(benzo[d][1,3]dioxol-5-yl)-2-(benzo[d]thiazol-2-yl)-3-(3,5-di-tert-butyl-4hydroxyphenyl)- $N, N$-dimethylpropanamide

$0.2 \mathrm{mmol}$ scale reaction; purified by petroleum ether/ ethyl acetate $=3: 1 ; 110 \mathrm{mg}$, white solid; $99 \%$ yield, $92: 8 \mathrm{dr}$, $98 \% / 17 \%$ ee; determined by HPLC analysis [Daicel chiralpak ID, $n$-hexane $/ i$-PrOH $\left.=90 / 10,1.0 \mathrm{~mL} / \mathrm{min}, \lambda=254 \mathrm{~nm}, \mathrm{t}_{1}=13.60 \mathrm{~min}, \mathrm{t}_{2}=16.04 \mathrm{~min}, \mathrm{t}_{3}=27.38 \mathrm{~min}, \mathrm{t}_{4}=31.27 \mathrm{~min}\right]$;

${ }^{1}$ H NMR (400 MHz, Chloroform- $\left.d\right) \delta 7.89-7.84(\mathrm{~m}, 1 \mathrm{H}), 7.75-7.73(\mathrm{~m}, 1 \mathrm{H}), 7.40-7.31(\mathrm{~m}$, 1H), $7.27-7.25(\mathrm{~m}, 1 \mathrm{H}), 7.16-7.14(\mathrm{~m}, 2 \mathrm{H}), 6.90-6.78(\mathrm{~m}, 2 \mathrm{H}), 6.77-6.50(\mathrm{~m}, 1 \mathrm{H}), 5.75-5.73$ $(\mathrm{m}, 2 \mathrm{H}), 5.36-5.33(\mathrm{~m}, 1 \mathrm{H}), 5.13(\mathrm{~s}, 1 \mathrm{H}), 4.90-4.79(\mathrm{~m}, 1 \mathrm{H}), 2.92(\mathrm{~s}, 3 \mathrm{H}), 2.74(\mathrm{~s}, 3 \mathrm{H}), 1.40(\mathrm{~s}$, $17 \mathrm{H}), 1.20(\mathrm{~s}, 1 \mathrm{H})$;

${ }^{13}$ C NMR (101 MHz, Chloroform- $d$ ) $\delta$ 169.7, 169.1, 152.4, 151.6, 147.4, 145.8, 135.8, 135.7, 134.8, 132.5, 125.4, 124.6, 124.1, 122.5, 121.5, 121.4, 109.1, 107.8, 100.6, 55.2, 53.2, 37.3, 35.9, 34.3, 30.2 ;

IR (neat): 2953, 1644, 1491, 1434, 1397, 1361, 1314, 1238, 1123, 1036, 862, 508, 760, $660 \mathrm{~cm}^{-1}$; HRMS (ESI) scaled for $[\mathrm{M}+\mathrm{Na}]^{+}: \mathrm{C}_{33} \mathrm{H}_{38} \mathrm{~N}_{2} \mathrm{O}_{4} \mathrm{SNa}, \mathrm{m} / \mathrm{z}$ : 581.2444, observed: 581.2438; $[\boldsymbol{\alpha}]_{\mathbf{D}}{ }^{20}=-44.8^{\circ}\left(c\right.$ 2.01, $\left.\mathrm{CH}_{2} \mathrm{Cl}_{2}\right)$;

m.p. $=55^{\circ} \mathrm{C}$.

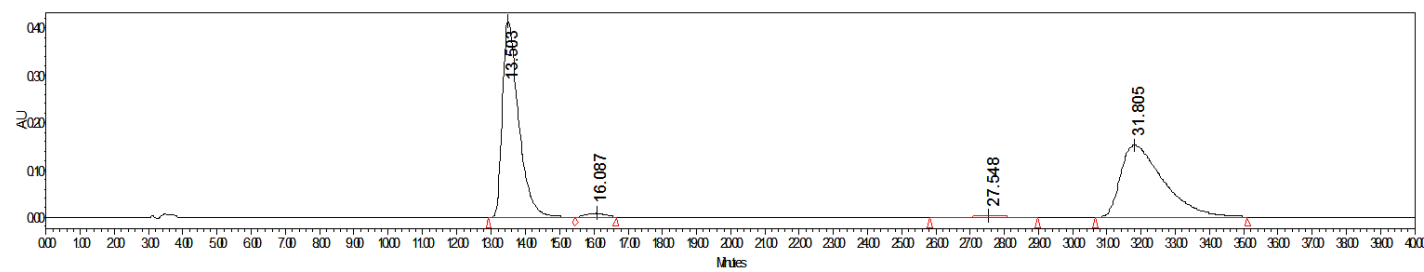

\begin{tabular}{|l|c|c|c|}
\hline & Retention Time & Area & \% Area \\
\hline 3 & 27.548 & 283700 & 1.03 \\
\hline 1 & 13.503 & 13626836 & 49.52 \\
\hline 2 & 16.087 & 314811 & 1.14 \\
\hline 4 & 31.805 & 13292714 & 48.31 \\
\hline
\end{tabular}

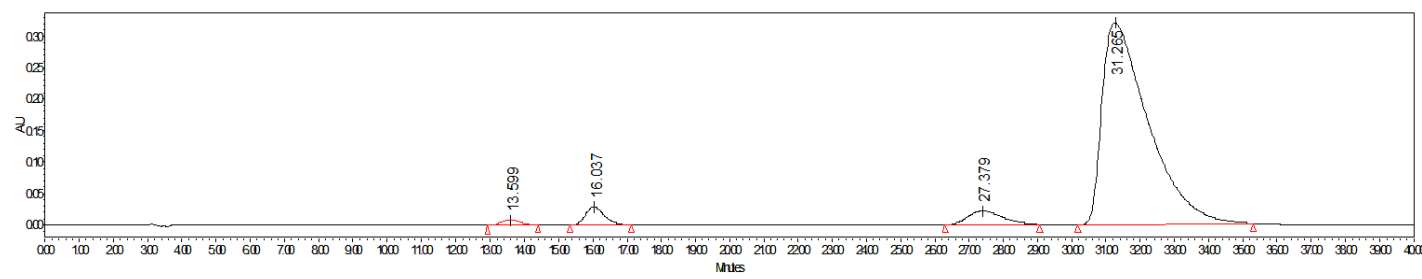

\begin{tabular}{|l|c|c|c|}
\hline & Retention Time & Area & \% Area \\
\hline 1 & 13.599 & 297484 & 0.90 \\
\hline 2 & 16.037 & 1071449 & 3.26 \\
\hline 3 & 27.379 & 1513088 & 4.60 \\
\hline 4 & 31.265 & 30019911 & 91.24 \\
\hline
\end{tabular}




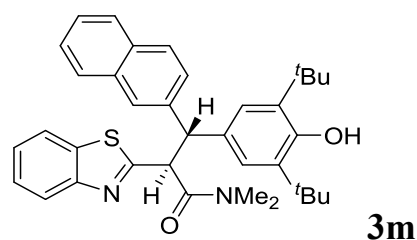

(2S,3R)-2-(benzo[d]thiazol-2-yl)-3-(3,5-di-tert-butyl-4-hydroxyphenyl)- $N, N$-dimethyl-3(naphthalen-2-yl)propenamide

$0.2 \mathrm{mmol}$ scale reaction; purified by petroleum ether/ ethyl acetate $=3: 1 ; 112.0 \mathrm{mg}$, white solid; $99 \%$ yield, 90:10 dr, 99\%/87\% ee; determined by HPLC analysis [Daicel chiralpak ADH, $n$-hexane $/ i$ $\left.\mathrm{PrOH}=80 / 20,1.0 \mathrm{~mL} / \mathrm{min}, \lambda=254 \mathrm{~nm}, \mathrm{t}_{1}=3.55 \mathrm{~min}, \mathrm{t}_{2}=6.13 \mathrm{~min}, \mathrm{t}_{3}=9.38 \mathrm{~min}, \mathrm{t}_{4}=11.36 \mathrm{~min}\right]$; ${ }^{1}$ H NMR $(400 \mathrm{MHz}$, Chloroform- $d$ ) $\delta 8.38-8.36(\mathrm{~m}, 1 \mathrm{H}), 7.91-7.88(\mathrm{~m}, 1 \mathrm{H}), 7.85-7.77(\mathrm{~m}, 1 \mathrm{H})$, $7.76-7.68(\mathrm{~m}, 1 \mathrm{H}), 7.64-7.53(\mathrm{~m}, 2 \mathrm{H}), 7.52-7.47$ (m, 1H), $7.44-7.30(\mathrm{~m}, 3 \mathrm{H}), 7.28(\mathrm{~s}, 2 \mathrm{H})$, $7.23-6.87(\mathrm{~m}, 1 \mathrm{H}), 5.77-5.74(\mathrm{~m}, 1 \mathrm{H}), 5.65-5.62(\mathrm{~m}, 1 \mathrm{H}), 5.10(\mathrm{~s}, 1 \mathrm{H}), 2.82(\mathrm{~s}, 3 \mathrm{H}), 2.77(\mathrm{~s}$, $3 \mathrm{H}), 1.38(\mathrm{~s}, 16 \mathrm{H}), 1.11(\mathrm{~s}, 2 \mathrm{H})$;

${ }^{13}$ C NMR (101 MHz, Chloroform- $d$ ) $\delta 170.1,169.9,152.6,151.4,136.0,135.8,135.6,132.0,131.9$, $131.4,128.6,127.1,126.0,125.4,125.2,124.8,124.7,124.5,123.6,122.3,121.5,53.2,50.3,37.3$, 36.0, 34.31, 30.3;

IR (neat): 3618, 3061, 2948, 2871, 1643, 1598, 1498, 1452, 1392, 1362, 1205, 765, 729, $690 \mathrm{~cm}^{-1}$; HRMS (ESI) scaled for $[\mathrm{M}+\mathrm{H}]^{+}: \mathrm{C}_{36} \mathrm{H}_{41} \mathrm{~N}_{2} \mathrm{O}_{2} \mathrm{~S}, \mathrm{~m} / \mathrm{z}$ : 565.2883, observed: 565.2882; $[\alpha]_{365^{23}}=-617.0^{\circ}\left(c 1.03, \mathrm{CH}_{2} \mathrm{Cl}_{2}\right)$; m.p. $=80^{\circ} \mathrm{C}$.

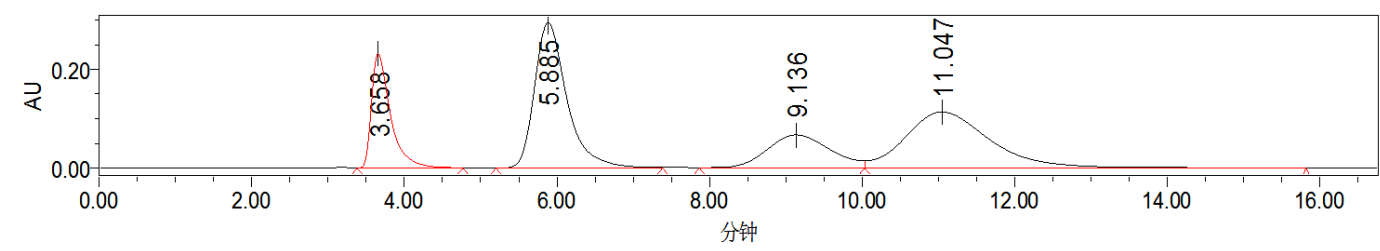

\begin{tabular}{|l|c|c|c|}
\hline & Retention Time & Area & \% Area \\
\hline 1 & 3.658 & 4028348 & 15.85 \\
\hline 2 & 5.885 & 8697685 & 34.22 \\
\hline 3 & 9.136 & 3871584 & 15.23 \\
\hline 4 & 11.047 & 8818790 & 34.70 \\
\hline
\end{tabular}

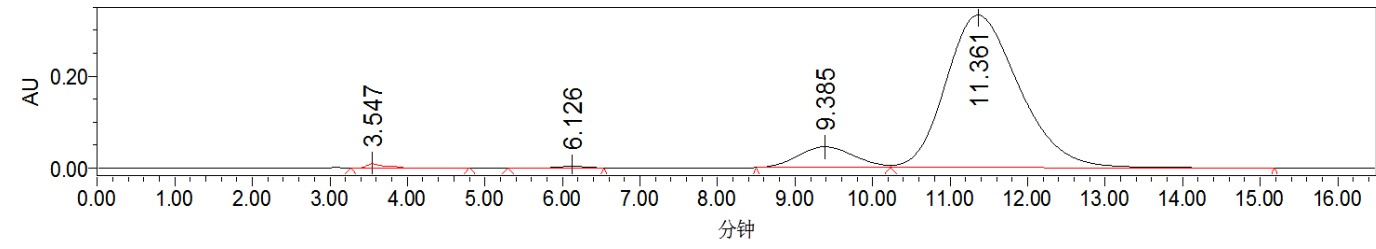

\begin{tabular}{|l|c|c|c|}
\hline & Retention Time & Area & \% Area \\
\hline 1 & 3.547 & 149826 & 0.62 \\
\hline 2 & 6.126 & 73250 & 0.30 \\
\hline 3 & 9.385 & 2279420 & 9.37 \\
\hline 4 & 11.361 & 21836796 & 89.72 \\
\hline
\end{tabular}




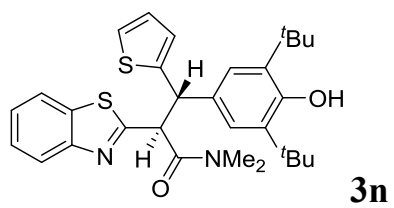

(2S,3S)-2-(benzo[d] thiazol-2-yl)-3-(3,5-di-tert-butyl-4-hydroxyphenyl)- $N, N$-dimethyl-3(thiophen-2-yl)propenamide

$0.2 \mathrm{mmol}$ scale reaction; purified by petroleum ether/ ethyl acetate $=3: 1 ; 103.8 \mathrm{mg}$, white solid; $99 \%$ yield, $91: 9 \mathrm{dr}, 96 \% / 17 \%$ ee; determined by HPLC analysis [Daicel chiralpak IA, $n$-hexane $/ i$-PrOH $\left.=90 / 10,1.0 \mathrm{~mL} / \mathrm{min}, \lambda=254 \mathrm{~nm}, \mathrm{t}_{1}=6.45 \mathrm{~min}, \mathrm{t}_{2}=7.28 \mathrm{~min}, \mathrm{t}_{3}=8.07 \mathrm{~min}, \mathrm{t}_{4}=13.62 \mathrm{~min}\right]$;

${ }^{1}$ H NMR (400 MHz, Chloroform- $d$ ) $\delta 7.92-7.88(\mathrm{~m}, 1 \mathrm{H}), 7.78-7.75(\mathrm{~m}, 1 \mathrm{H}), 7.41-7.26(\mathrm{~m}$, $3 \mathrm{H}), 7.18-6.90(\mathrm{~m}, 2 \mathrm{H}), 6.85-6.84(\mathrm{~m}, 1 \mathrm{H}), 6.70-6.67(\mathrm{~m}, 1 \mathrm{H}), 5.27(\mathrm{~s}, 1 \mathrm{H}), 5.17-5.12(\mathrm{~m}$, $2 \mathrm{H}), 2.90(\mathrm{~s}, 3 \mathrm{H}), 2.71(\mathrm{~s}, 3 \mathrm{H}), 1.43(\mathrm{~s}, 16 \mathrm{H}), 1.21(\mathrm{~s}, 2 \mathrm{H})$;

${ }^{13}$ C NMR (101 MHz, Chloroform- $d$ ) $\delta 169.4,168.7,152.7,151,7,144.3,135.8,135.7,131.7,126.3$, 125.6, 125.5, 124.8, 124.5 124.1, 122.6, 121.6, 55.1, 51.6, 37.4, 35.9, 34.4, 30.3;

IR (neat): 3620, 3088, 2950, 2869, 1645, 1557, 1481, 1455, 1394, 1361, 1202, 757, 726, $695 \mathrm{~cm}^{-1}$; HRMS (ESI) scaled for [M+Na] $]^{+} \mathrm{C}_{30} \mathrm{H}_{36} \mathrm{~N}_{2} \mathrm{O}_{2} \mathrm{~S}_{2} \mathrm{Na}, \mathrm{m} / \mathrm{z}$ : 543.2110, observed: 543.2118; $[\alpha]_{546^{23}}=-47.1^{\circ}\left(c 0.92, \mathrm{CH}_{2} \mathrm{Cl}_{2}\right)$; m.p. $=55^{\circ} \mathrm{C}$.

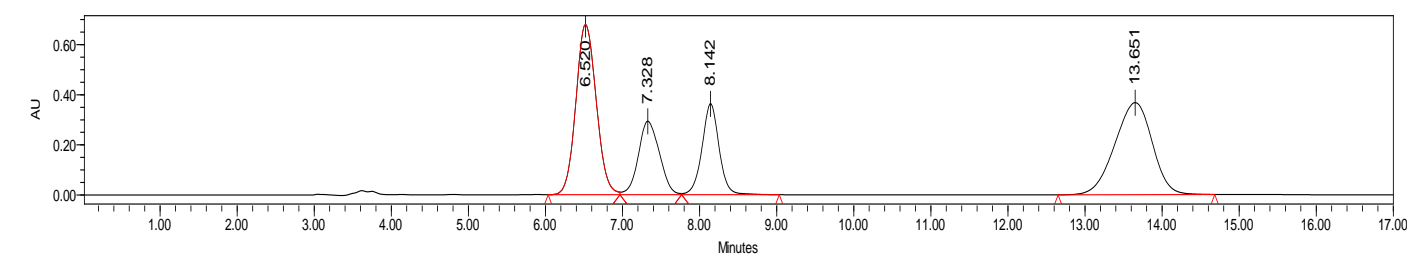

\begin{tabular}{|l|c|c|c|}
\hline & Retention Time & Area & \% Area \\
\hline 1 & 6.520 & 12473045 & 34.57 \\
\hline 2 & 7.328 & 5581360 & 15.47 \\
\hline 3 & 8.142 & 5606539 & 15.54 \\
\hline 4 & 13.651 & 12414735 & 34.41 \\
\hline
\end{tabular}

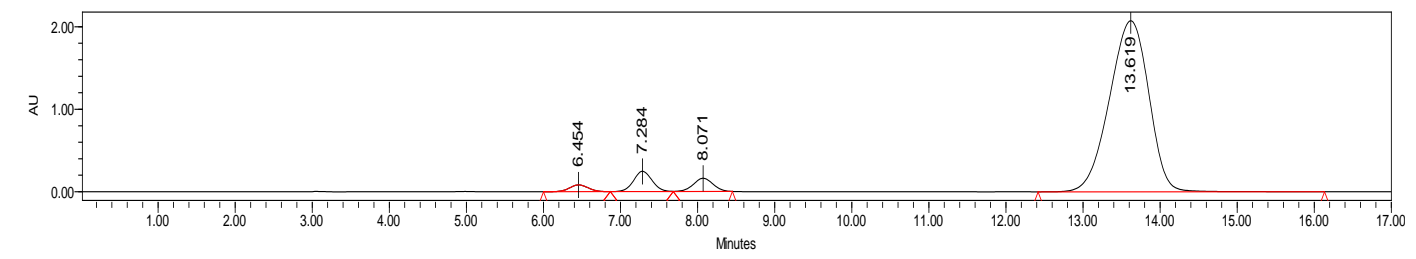

\begin{tabular}{|l|c|c|c|}
\hline & Retention Time & Area & \% Area \\
\hline 1 & 6.454 & 1392465 & 1.70 \\
\hline 2 & 7.284 & 4121612 & 5.03 \\
\hline 3 & 8.071 & 2851391 & 3.48 \\
\hline 4 & 13.619 & 73515463 & 89.78 \\
\hline
\end{tabular}




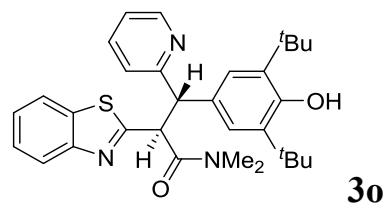

(2S,3S)-2-(benzo[d] thiazol-2-yl)-3-(3,5-di-tert-butyl-4-hydroxyphenyl)- $N, N$-dimethyl-3(pyridin-2-yl)propenamide

$0.2 \mathrm{mmol}$ scale reaction; purified by petroleum ether/ ethyl acetate $=3: 1 ; 102 \mathrm{mg}$, white solid; $99 \%$ yield, $97: 3 \mathrm{dr}$, 95\%/82\% ee; determined by HPLC analysis [Daicel chiralpak IF, $n$-hexane $/ i$-PrOH $\left.=90 / 10,1.0 \mathrm{~mL} / \mathrm{min}, \lambda=254 \mathrm{~nm}, \mathrm{t}_{1}=12.96 \mathrm{~min}, \mathrm{t}_{2}=16.00 \mathrm{~min}, \mathrm{t}_{3}=34.26 \mathrm{~min}, \mathrm{t}_{4}=39.82 \mathrm{~min}\right]$;

${ }^{1} \mathbf{H}$ NMR (400 MHz, Chloroform- $d$ ) $\delta 8.55-8.49(\mathrm{~m}, 1 \mathrm{H}), 7.93-7.83(\mathrm{~m}, 1 \mathrm{H}), 7.77-7.69(\mathrm{~m}$, $1 \mathrm{H}), 7.52-7.41(\mathrm{~m}, 2 \mathrm{H}), 7.37-7.27(\mathrm{~m}, 2 \mathrm{H}), 7.25-7.00(\mathrm{~m}, 2 \mathrm{H}), 6.93-6.83(\mathrm{~m}, 1 \mathrm{H}), 5.81-5.78$ (m, 1H), $5.13(\mathrm{~s}, 1 \mathrm{H}), 4.97-4.94(\mathrm{~m}, 1 \mathrm{H}), 2.87(\mathrm{~s}, 3 \mathrm{H}), 2.70(\mathrm{~s}, 3 \mathrm{H}), 1.40(\mathrm{~s}, 17 \mathrm{H}), 1.13(\mathrm{~s}, 1 \mathrm{H})$; ${ }^{13}$ C NMR (101 MHz, Chloroform- $d$ ) $\delta 169.9,168.9,159.9,152.7,151.9,148.8,136.1,135.6,135.5$, $130.8,125.3,125.2,124.4,124.3,122.7,121.2,56.9,52.7,37.5,35.7,34.3,30.3$;

IR (neat): $3610 \mathrm{~cm}^{-1}, 3369,3058,2951,2870,1633,1588,1502,1471,1394,1361,1212,760,726$, $699 \mathrm{~cm}^{-1}$;

HRMS (ESI) scaled for $[\mathrm{M}+\mathrm{H}]^{+}: \mathrm{C}_{31} \mathrm{H}_{38} \mathrm{~N}_{3} \mathrm{O}_{2} \mathrm{~S}, \mathrm{~m} / \mathrm{z}: 516.2679$, observed: 516.2669;

$[\alpha]_{436^{23}}=272.4^{\circ}\left(\right.$ c 2.14, $\left.\mathrm{CH}_{2} \mathrm{Cl}_{2}\right)$;

m.p. $=57^{\circ} \mathrm{C}$.

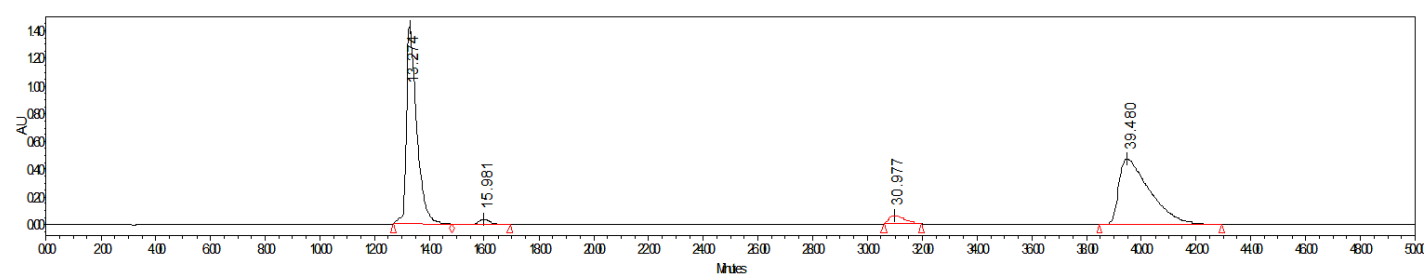

\begin{tabular}{|l|c|c|c|}
\hline & Retention Time & Area & \% Area \\
\hline 1 & 13.274 & 37681283 & 48.20 \\
\hline 2 & 15.981 & 1052133 & 1.35 \\
\hline 3 & 30.977 & 2340334 & 2.99 \\
\hline 4 & 39.480 & 37099523 & 47.46 \\
\hline
\end{tabular}

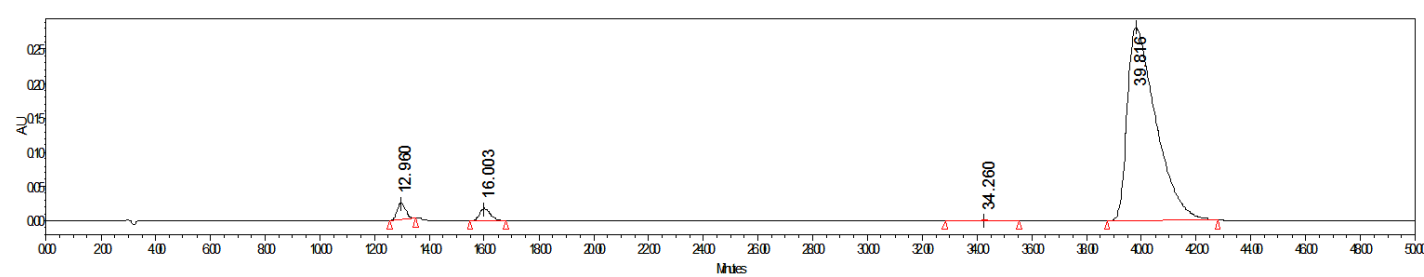

\begin{tabular}{|l|c|c|c|}
\hline & Retention Time & Area & \% Area \\
\hline 1 & 12.960 & 523928 & 2.43 \\
\hline 2 & 16.003 & 496887 & 2.31 \\
\hline 3 & 34.260 & 74325 & 0.35 \\
\hline 4 & 39.816 & 20447880 & 94.92 \\
\hline
\end{tabular}




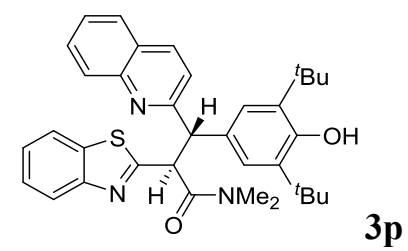

(2S,3S)-2-(benzo[d] thiazol-2-yl)-3-(3,5-di-tert-butyl-4-hydroxyphenyl)- $N, N$-dimethyl-3(quinolin-2-yl)propenamide

$0.2 \mathrm{mmol}$ scale reaction; purified by petroleum ether/ ethyl acetate $=3: 1 ; 112 \mathrm{mg}$, white solid; $99 \%$ yield, $87: 13 \mathrm{dr}$, $99 \% / 1 \%$ ee; determined by HPLC analysis [Daicel chiralpak ID, $n$-hexane $/ i$-PrOH $\left.=90 / 10,1.0 \mathrm{~mL} / \mathrm{min}, \lambda=254 \mathrm{~nm}, \mathrm{t}_{1}=17.41 \mathrm{~min}, \mathrm{t}_{2}=26.52 \mathrm{~min}, \mathrm{t}_{3}=29.18 \mathrm{~min}, \mathrm{t}_{4}=44.44 \mathrm{~min}\right]$;

${ }^{1}$ H NMR (400 MHz, Chloroform- $d$ ) $\delta 8.11-8.02(\mathrm{~m}, 1 \mathrm{H}), 7.97-7.80(\mathrm{~m}, 2 \mathrm{H}), 7.79-7.53(\mathrm{~m}$, $3 \mathrm{H}), 7.54-7.43(\mathrm{~m}, 2 \mathrm{H}), 7.38-7.30(\mathrm{~m}, 2 \mathrm{H}), 7.29-7.24(\mathrm{~m}, 1 \mathrm{H}), 7.18-6.87(\mathrm{~m}, 1 \mathrm{H}), 5.99-5.96$ (m, 1H), $5.21-5.18(\mathrm{~m}, 1 \mathrm{H}), 5.12(\mathrm{~s}, 1 \mathrm{H}), 2.95(\mathrm{~s}, 3 \mathrm{H}), 2.71(\mathrm{~s}, 3 \mathrm{H}), 1.39(\mathrm{~s}, 16 \mathrm{H}), 1.11(\mathrm{~s}, 2 \mathrm{H})$;

${ }^{13}$ C NMR (101 MHz, Chloroform- $d$ ) $\delta 169.8,169.1,160.3,152.8,152.0,147.6,136.0,135.5,135.4$, 130.4, 129.3, 128.9, 127.2, 126.6, 125.6, 125.4, 125.3, 124.4, 122.6, 121.2, 57.0, 52.6, 37.6, 35.7, $34.3,30.3$;

IR (neat): 3626, 2952, 1641, 1498, 1431, 1395, 1234, 1120, $755 \mathrm{~cm}^{-1}$;

HRMS (ESI) scaled for $[\mathrm{M}+\mathrm{H}]^{+}: \mathrm{C}_{35} \mathrm{H}_{40} \mathrm{~N}_{3} \mathrm{O}_{2} \mathrm{~S}, \mathrm{~m} / \mathrm{z}$ : 566.2836, observed: 566.2831;

$[\boldsymbol{\alpha}]_{\mathbf{D}}{ }^{\mathbf{2 0}}=192.1^{\circ}\left(c\right.$ 2.12, $\left.\mathrm{CH}_{2} \mathrm{Cl}_{2}\right)$;

m.p. $=110^{\circ} \mathrm{C}$.

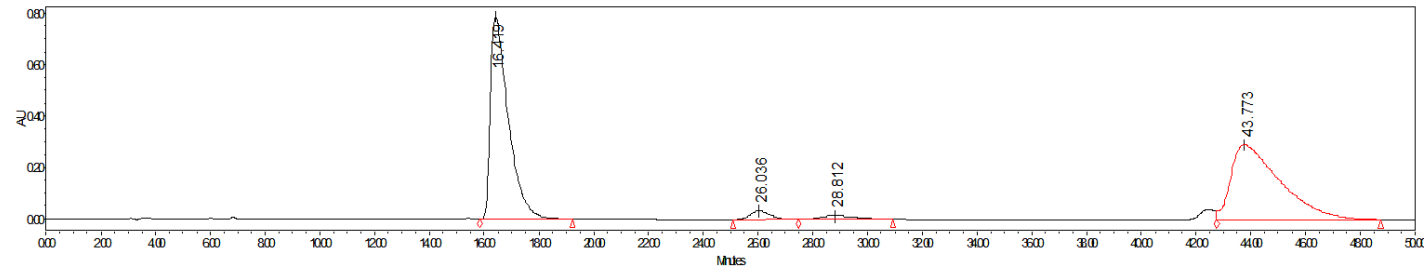

\begin{tabular}{|l|c|c|c|}
\hline & Retention Time & Area & $\%$ Area \\
\hline 1 & 16.419 & 36005527 & 47.50 \\
\hline 2 & 26.036 & 1772535 & 2.34 \\
\hline 3 & 28.812 & 1392238 & 1.84 \\
\hline 4 & 43.773 & 36631211 & 48.33 \\
\hline
\end{tabular}

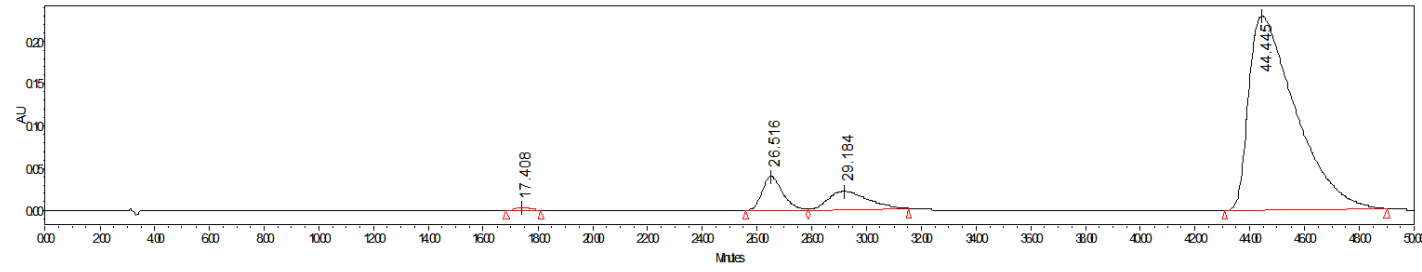

\begin{tabular}{|l|c|c|c|}
\hline & Retention Time & Area & $\%$ Area \\
\hline 1 & 17.408 & 127163 & 0.40 \\
\hline 2 & 26.516 & 2062711 & 6.55 \\
\hline 3 & 29.184 & 2114109 & 6.72 \\
\hline 4 & 44.445 & 27175166 & 86.33 \\
\hline
\end{tabular}




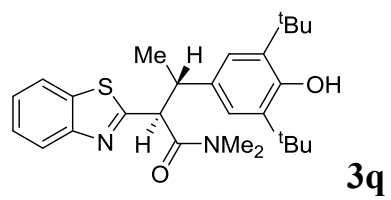

(2S,3S)-2-(benzo[d]thiazol-2-yl)-3-(3,5-di-tert-butyl-4-hydroxyphenyl)- $N, N$ -

\section{dimethylbutanamide}

$0.2 \mathrm{mmol}$ scale reaction; purified by petroleum ether/ ethyl acetate $=3: 1 ; 85 \mathrm{mg}$, white solid; $94 \%$ yield, $71: 29 \mathrm{dr}$, 94\%/92\% ee; determined by HPLC analysis [Daicel chiralpak IF, $n$-hexane $/ i$-PrOH $\left.=95 / 5,1.0 \mathrm{~mL} / \mathrm{min}, \lambda=254 \mathrm{~nm}, \mathrm{t}_{1}=8.63 \mathrm{~min}, \mathrm{t}_{2}=9.33 \mathrm{~min}, \mathrm{t}_{3}=15.73 \mathrm{~min}, \mathrm{t}_{4}=28.39 \mathrm{~min}\right]$;

${ }^{1} \mathbf{H}$ NMR (400 MHz, Chloroform- $d$ ) $\delta 8.04-7.74(\mathrm{~m}, 2 \mathrm{H}), 7.50-7.27$ (m, 2H), $7.20-6.90(\mathrm{~m}, 2 \mathrm{H})$, $4.96(\mathrm{~s}, 1 \mathrm{H}), 4.63-4,56(\mathrm{~m}, 1 \mathrm{H}), 3.72-3.50(\mathrm{~m}, 1 \mathrm{H}), 3.00-2.60(\mathrm{~m}, 1 \mathrm{H}), 2.80(\mathrm{~m}, 6 \mathrm{H}), 1.35(\mathrm{~m}$, $21 \mathrm{H})$;

${ }^{13}$ C NMR (101 MHz, Chloroform- $d$ ) $\delta 170.5,169.1,152.2,152.0,135.6,135.3,132.8,125.5,124.6$, 124.0, 122.4, 121.3, 55.2, 45.2, 37.6, 36.0, 34.1, 30.1, 18.9;

IR (neat): 3635, 2955, 1638, 1455, 1434, 1397, 1314, 1235, 1120, 932, 759, 702, $640 \mathrm{~cm}^{-1}$;

HRMS (ESI) scaled for $[\mathrm{M}+\mathrm{H}]^{+}: \mathrm{C}_{27} \mathrm{H}_{37} \mathrm{~N}_{2} \mathrm{O}_{2} \mathrm{~S}, \mathrm{~m} / \mathrm{z}$ : 453.2570, observed: 453.2573; $[\boldsymbol{\alpha}]_{\mathbf{D}} \mathbf{D}^{\mathbf{2 0}}=-12.1^{\circ}\left(c 0.1 .49, \mathrm{CH}_{2} \mathrm{Cl}_{2}\right)$;

m.p. $=65^{\circ} \mathrm{C}$.

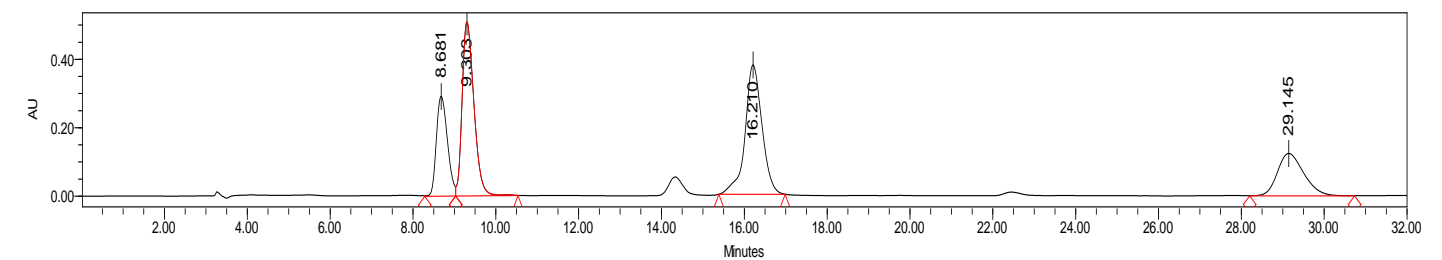

\begin{tabular}{|l|c|c|c|}
\hline & Retention Time & Area & \% Area \\
\hline 1 & 8.681 & 5428092 & 17.09 \\
\hline 2 & 9.303 & 10059186 & 31.67 \\
\hline 3 & 16.210 & 10761820 & 33.88 \\
\hline 4 & 29.145 & 5515319 & 17.36 \\
\hline
\end{tabular}

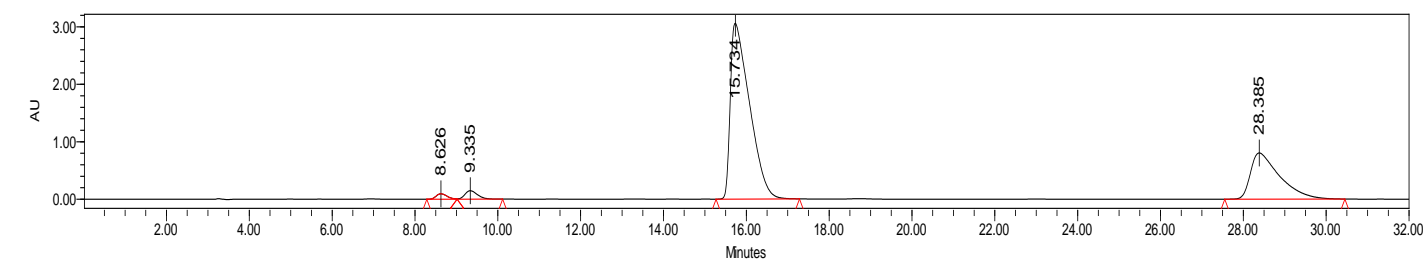

\begin{tabular}{|l|c|c|c|}
\hline & Retention Time & Area & \% Area \\
\hline 1 & 8.626 & 1731701 & 1.22 \\
\hline 2 & 9.335 & 3105103 & 2.18 \\
\hline 3 & 15.734 & 98282663 & 69.02 \\
\hline 4 & 28.385 & 39282286 & 27.59 \\
\hline
\end{tabular}




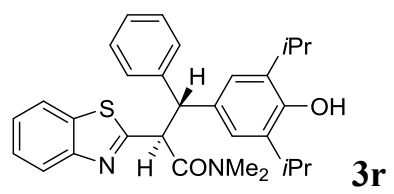

(2S,3R)-2-(benzo[d]thiazol-2-yl)-3-(4-hydroxy-3,5-diisopropylphenyl)- $N, N$-dimethyl-3phenylpropanamide

$0.2 \mathrm{mmol}$ scale reaction; purified by petroleum ether/ ethyl acetate $=3: 1 ; 97 \mathrm{mg}$, white solid; $99 \%$ yield, $81: 19 \mathrm{dr}, 96 \% / 40 \%$ ee; determined by HPLC analysis [Daicel chiralpak IA, $n$-hexane $/ i-\mathrm{PrOH}$ $\left.=90 / 10,1.0 \mathrm{~mL} / \mathrm{min}, \lambda=254 \mathrm{~nm}, \mathrm{t}_{1}=10.01 \mathrm{~min}, \mathrm{t}_{2}=11.85 \mathrm{~min}, \mathrm{t}_{3}=16.73 \mathrm{~min}, \mathrm{t}_{4}=35.82 \mathrm{~min}\right]$; ${ }^{1}$ H NMR $(400 \mathrm{MHz}$, Chloroform- $d) \delta 7.86-7.83(\mathrm{~m}, 1 \mathrm{H}), 7.75-7.68(\mathrm{~m}, 1 \mathrm{H}), 7.39-7.30(\mathrm{~m}, 3 \mathrm{H})$, $7.30-7.18(\mathrm{~m}, 2 \mathrm{H}), 7.13-7.03(\mathrm{~m}, 3 \mathrm{H}), 7.00-6.93(\mathrm{~m}, 1 \mathrm{H}), 5.48(\mathrm{~d}, J=12.0 \mathrm{~Hz}, 1 \mathrm{H}), 5.32(\mathrm{~s}, 1 \mathrm{H})$, $4.96-4.92$ (m, 1H), $3.20-3.14$ (m, 2H), 2.97 (s, 3H), 2.74 (s, 3H), $1.23-1.19$ (m, 10H), $1.07-1.03$ (m, 2H);

${ }^{13}$ C NMR (101 MHz, Chloroform- $d$ ) $\delta 169.8,169.2,151.5,148.8,141.0,135.7,133.9,133.7,128.4$, 128.2, 126.2, 125.5, 124.7, 122.8, 122.4, 121.5, 55.4, 53.0, 37.5, 36.0, 27.1, 22.9, 22.8;

IR (neat): 3628, 3055, 2950, 2872, 1645, 1599, 1495, 1451, 1393, 1359, 1208, 759, 729, $696 \mathrm{~cm}^{-1}$; HRMS (ESI) scaled for $[\mathrm{M}+\mathrm{H}]^{+}: \mathrm{C}_{30} \mathrm{H}_{34} \mathrm{~N}_{2} \mathrm{O}_{2} \mathrm{~S}, \mathrm{~m} / \mathrm{z}: 487.2419$, observed: 487.2425; m.p. $=95^{\circ} \mathrm{C}$.

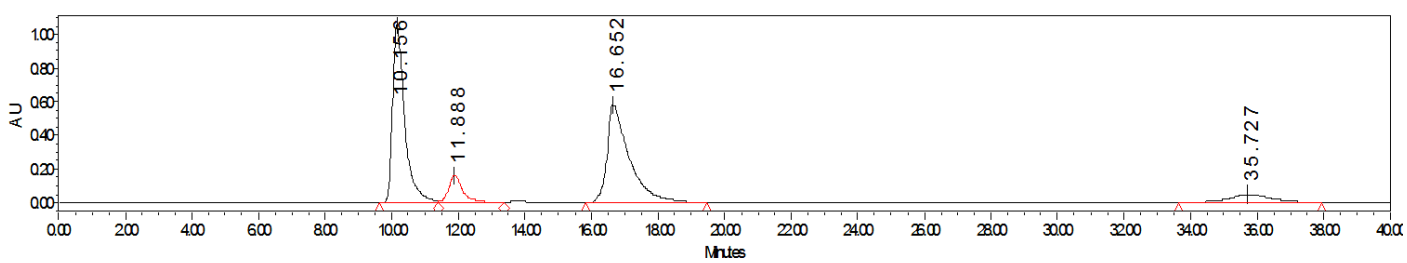

\begin{tabular}{|l|c|c|c|}
\hline & Retention Time & Area & \% Area \\
\hline 1 & 10.156 & 26712780 & 42.85 \\
\hline 2 & 11.888 & 4769019 & 7.65 \\
\hline 3 & 16.652 & 26765719 & 42.94 \\
\hline 4 & 35.727 & 4088784 & 6.56 \\
\hline
\end{tabular}

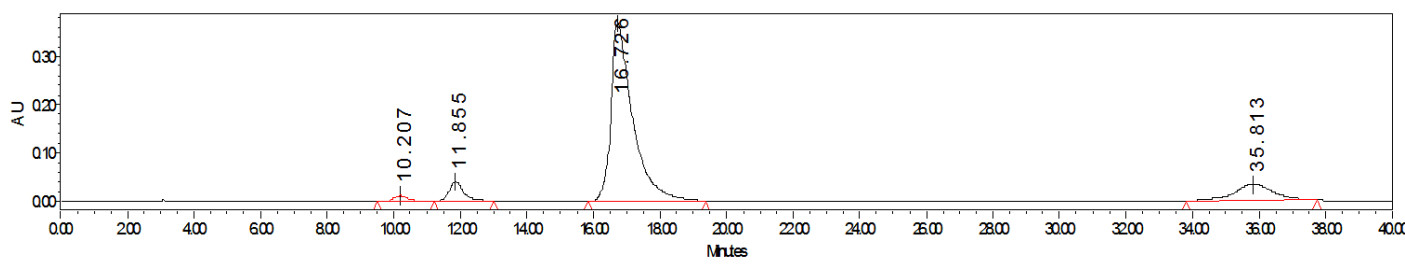

\begin{tabular}{|l|c|c|c|}
\hline & Retention Time & Area & \% Area \\
\hline 1 & 10.207 & 294404 & 1.44 \\
\hline 2 & 11.855 & 1150172 & 5.61 \\
\hline 3 & 16.726 & 16665589 & 81.33 \\
\hline 4 & 35.819 & 2382049 & 11.62 \\
\hline
\end{tabular}




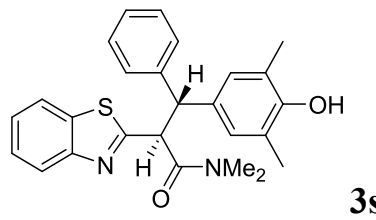

(2S,3R)-2-(benzo[d] thiazol-2-yl)-3-(4-hydroxy-3,5-dimethylphenyl)- $N, N$-dimethyl-3phenylpropanamide

$0.2 \mathrm{mmol}$ scale reaction; purified by petroleum ether/ ethyl acetate $=3: 1 ; 84 \mathrm{mg}$, white solid; $98 \%$ yield, $56: 44 \mathrm{dr}, 87 \% / 89 \%$ ee; determined by HPLC analysis [Daicel chiralpak ID, $n$-hexane $/ i$-PrOH $\left.=80 / 20,1.0 \mathrm{~mL} / \mathrm{min}, \lambda=254 \mathrm{~nm}, \mathrm{t}_{1}=14.26 \mathrm{~min}, \mathrm{t}_{2}=17.33 \mathrm{~min}, \mathrm{t}_{3}=19.57 \mathrm{~min}, \mathrm{t}_{4}=25.21 \mathrm{~min}\right]$;

${ }^{1}$ H NMR (400 MHz, Chloroform- $\left.d\right) \delta 7.91-7.83(\mathrm{~m}, 1 \mathrm{H}), 7.74-7.71(\mathrm{~m}, 1 \mathrm{H}), 7.39-7.26(\mathrm{~m}$, 5H), $7.25-7.14(\mathrm{~m}, 1 \mathrm{H}), 7.10-6.94(\mathrm{~m}, 3 \mathrm{H}), 5.51-5.45(\mathrm{~m}, 1 \mathrm{H}), 5.47-5.20(\mathrm{~m}, 1 \mathrm{H}), 4.91-4.87$ (m, 1H), $3.03(\mathrm{~s}, 1 \mathrm{H}), 2.98(\mathrm{~s}, 2 \mathrm{H}), 2.74(\mathrm{~s}, 1 \mathrm{H}), 2.70(\mathrm{~s}, 2 \mathrm{H}), 2.19(\mathrm{~s}, 2 \mathrm{H}), 2.06(\mathrm{~s}, 4 \mathrm{H})$;

${ }^{13}$ C NMR (101 MHz, Chloroform- $d$ ) $\delta$ 169.6, 169.4, 151.6, 150.9, 142.7, 135.7, 131.8, 128.4, 128.3, $127.7,126.6,125.5,123.1,121.6,54.8,52.4,37.5,35.9,16.1$;

IR (neat): 3317, 2918, 2360, 1636, 1487, 1441, 1399, 1203, 1144, 758, 729, $698 \mathrm{~cm}^{-1}$;

HRMS (ESI) scaled for [M+Na] ${ }^{+}: \mathrm{C}_{26} \mathrm{H}_{26} \mathrm{~N}_{2} \mathrm{O}_{2} \mathrm{Na}, \mathrm{m} / \mathrm{z}$ : 453.1607, observed: 453.1611;

$[\alpha]_{\mathbf{D}}{ }^{20}=-54.6^{\circ}\left(c 0.56, \mathrm{CH}_{2} \mathrm{Cl}_{2}\right)$;

m.p. $=120^{\circ} \mathrm{C}$.

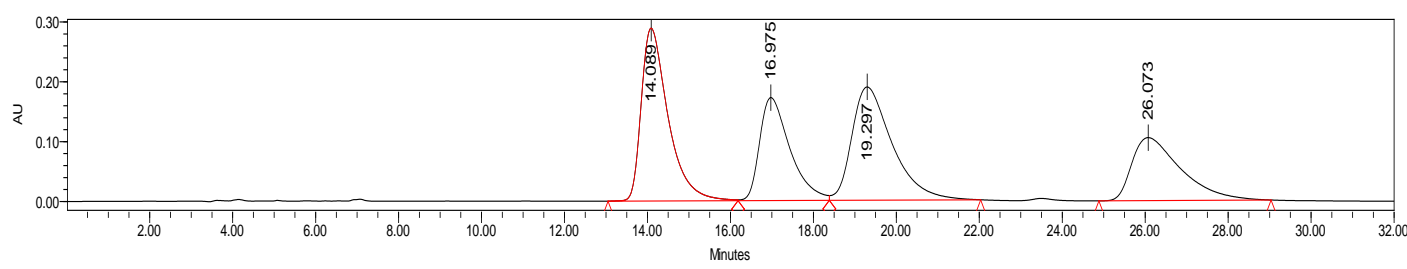

\begin{tabular}{|l|c|c|c|}
\hline & Retention Time & Area & \% Area \\
\hline 1 & 14.089 & 12986683 & 30.07 \\
\hline 2 & 16.975 & 8752741 & 20.27 \\
\hline 3 & 19.297 & 12820072 & 29.69 \\
\hline 4 & 26.073 & 8625587 & 19.97 \\
\hline
\end{tabular}

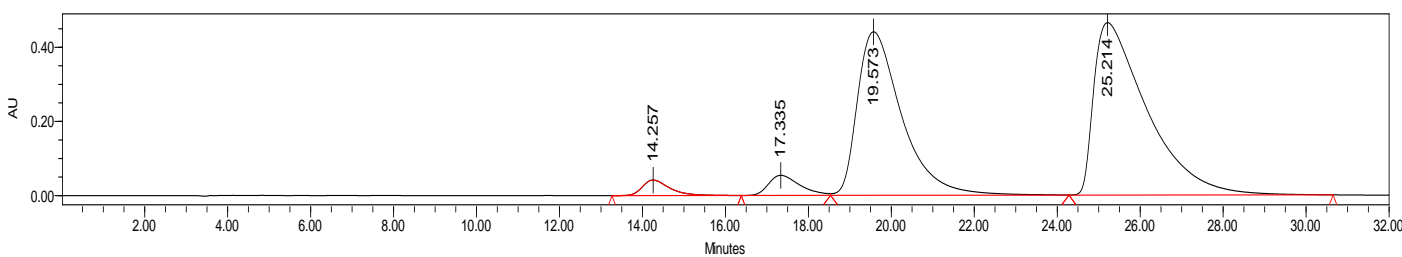

\begin{tabular}{|l|c|c|c|}
\hline & Retention Time & Area & \% Area \\
\hline 1 & 14.257 & 1909932 & 2.41 \\
\hline 2 & 17.335 & 2854704 & 3.61 \\
\hline 3 & 19.573 & 32848187 & 41.52 \\
\hline 4 & 25.214 & 41499813 & 52.46 \\
\hline
\end{tabular}




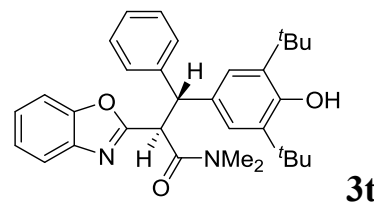

(2R,3R)-2-(benzo[d]oxazol-2-yl)-3-(3,5-di-tert-butyl-4-hydroxyphenyl)- $N, N$-dimethyl-3phenylpropanamide

$0.2 \mathrm{mmol}$ scale reaction; purified by petroleum ether/ ethyl acetate $=3: 1 ; 99.4 \mathrm{mg}$, white solid; $99 \%$ yield, $91: 9 \mathrm{dr}, 97 \% / 37 \%$ ee; determined by HPLC analysis [Daicel chiralpak ADH, $n$-hexane $/ i$ $\mathrm{PrOH}=90 / 10,1.0 \mathrm{~mL} / \mathrm{min}, \lambda=254 \mathrm{~nm}, \mathrm{t}_{1}=5.87 \mathrm{~min}, \mathrm{t}_{2}=7.48 \mathrm{~min}, \mathrm{t}_{3}=10.52 \mathrm{~min}, \mathrm{t}_{4}=16.98$ $\min ]$;

${ }^{1}$ H NMR (400 MHz, Chloroform- $d$ ) $\delta 7.58-7.53(\mathrm{~m}, 1 \mathrm{H}), 7.47-7.41(\mathrm{~m}, 1 \mathrm{H}), 7.33-7.28(\mathrm{~m}$, 2H), $7.24-7.09(\mathrm{~m}, 6 \mathrm{H}), 7.03-6.91(\mathrm{~m}, 1 \mathrm{H}), 5.27-5.16(\mathrm{~m}, 2 \mathrm{H}), 5.12(\mathrm{~s}, 1 \mathrm{H}), 2.97(\mathrm{~s}, 3 \mathrm{H}), 2.78$ (s, 3H), $1.40(\mathrm{~s}, 16 \mathrm{H}), 1.17(\mathrm{~s}, 2 \mathrm{H})$;

${ }^{13}$ C NMR (101 MHz, Chloroform- $d$ ) $\delta 167.6,162.5,152.5,150.8,141.3,140.6,135.6,132.0,128.3$, 127.9, 126.3, 124.6, 124.4, 124.0, 119.6, 110.7, 51.7, 48.2, 37.4, 35.9, 34.3, 30.2;

IR (neat): 3370, 3062, 2951, 2869, 1639, 1565, 1496, 1452, 1397, 1360, 1219, 749, 730, $658 \mathrm{~cm}^{-1}$; HRMS (ESI) scaled for $[\mathrm{M}+\mathrm{H}]^{+}: \mathrm{C}_{32} \mathrm{H}_{39} \mathrm{~N}_{2} \mathrm{O}_{3}, \mathrm{~m} / \mathrm{z}$ : 499.2955, observed: 499.2958;

$[\alpha]_{365^{23}}=-139.5^{\circ}\left(c 1.51, \mathrm{CH}_{2} \mathrm{Cl}_{2}\right)$;

m.p. $=35^{\circ} \mathrm{C}$.

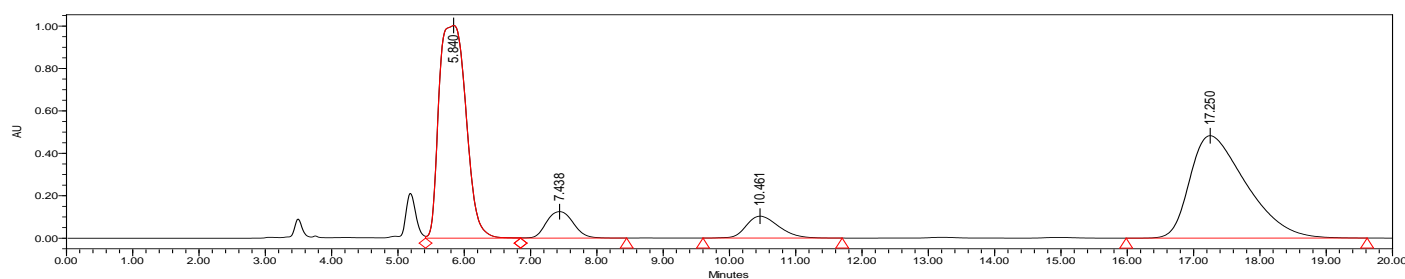

\begin{tabular}{|l|c|c|c|}
\hline & Retention Time & Area & \% Area \\
\hline 1 & 5.840 & 28339377 & 44.41 \\
\hline 2 & 7.438 & 3521614 & 5.52 \\
\hline 3 & 10.461 & 3516681 & 5.51 \\
\hline 4 & 17.250 & 28440229 & 44.56 \\
\hline
\end{tabular}

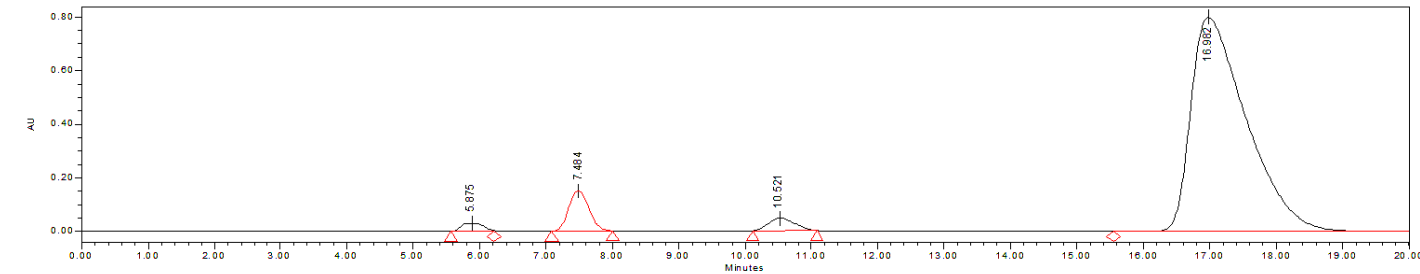

\begin{tabular}{|l|c|c|c|}
\hline & Retention Time & Area & $\%$ Area \\
\hline 1 & 5.875 & 688518 & 1.34 \\
\hline 2 & 7.484 & 3155786 & 6.14 \\
\hline 3 & 10.521 & 1281429 & 2.50 \\
\hline 4 & 16.982 & 46230521 & 90.02 \\
\hline
\end{tabular}




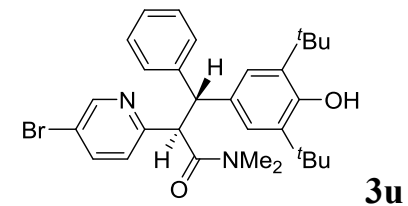

(2R,3R)-2-(5-bromopyridin-2-yl)-3-(3,5-di-tert-butyl-4-hydroxyphenyl)- $N, N$-dimethyl-3phenylpropanamide

$0.2 \mathrm{mmol}$ scale reaction; purified by petroleum ether/ ethyl acetate $=3: 1 ; 100 \mathrm{mg}$, white solid; $93 \%$ yield, $78: 22 \mathrm{dr}, 96 \% / 57 \%$ ee; determined by HPLC analysis [Daicel chiralpak IE, $n$-hexane $/ i-\mathrm{PrOH}$ $\left.=90 / 10,1.0 \mathrm{~mL} / \mathrm{min}, \lambda=254 \mathrm{~nm}, \mathrm{t}_{1}=8.70 \mathrm{~min}, \mathrm{t}_{2}=9.60 \mathrm{~min}, \mathrm{t}_{3}=10.13 \mathrm{~min}, \mathrm{t}_{4}=10.68 \mathrm{~min}\right]$;

${ }^{1}$ H NMR (400 MHz, Chloroform- $d$ ) $\delta 8.41-8.38(\mathrm{~m}, 1 \mathrm{H}), 7.61-7.57(\mathrm{~m}, 1 \mathrm{H}), 7.51-7.28(\mathrm{~m}$, 2H), $7.20-7.15(\mathrm{~m}, 2 \mathrm{H}), 7.15-7.08(\mathrm{~m}, 3 \mathrm{H}), 7.04-6.79(\mathrm{~m}, 1 \mathrm{H}), 5.08(\mathrm{~s}, 1 \mathrm{H}), 5.02-4.99(\mathrm{~m}$, $1 \mathrm{H}), 4.86-4.83(\mathrm{~m}, 1 \mathrm{H}), 2.97(\mathrm{~s}, 1 \mathrm{H}), 2.91(\mathrm{~s}, 2 \mathrm{H}), 2.75-2.74(\mathrm{~m}, 3 \mathrm{H}), 1.39(\mathrm{~s}, 14 \mathrm{H}), 1.28(\mathrm{~s}, 4 \mathrm{H})$; ${ }^{13}$ C NMR (101 MHz, Chloroform- $d$ ) $\delta 171.3,157.3,152.3,149.2,141.6,138.9,135.5,133.0,128.7$, 128.3, 128.1, 127.7, 126.0, 124.8, 124.3, 118.8, 55.4, 53.9, 37.3, 35.8, 34.3, 30.3;

IR (neat): 3844, 2360, 1635, 1511, 1437, 1390, 1362, 1451, 1393, 1359, 1197, 1112, 1004, 730, 699, $634 \mathrm{~cm}^{-1}$;

HRMS (ESI) scaled for $[\mathrm{M}+\mathrm{H}]^{+}: \mathrm{C}_{30} \mathrm{H}_{38} \mathrm{BrN}_{2} \mathrm{O}_{2}, \mathrm{~m} / \mathrm{z}: 537.2111,539.2091$, observed: 537.2109, 539.2090;

$[\boldsymbol{\alpha}]_{\mathbf{D}} \mathbf{2 0}^{\mathbf{2 0}}=-3.1^{\circ}\left(c 1.92, \mathrm{CH}_{2} \mathrm{Cl}_{2}\right)$;

m.p. $=85^{\circ} \mathrm{C}$.

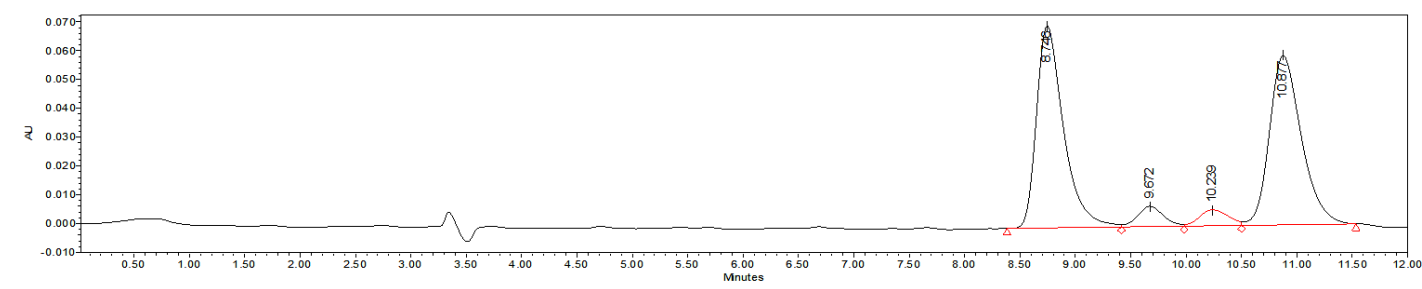

\begin{tabular}{|l|c|c|c|}
\hline & Retention Time & Area & \% Area \\
\hline 1 & 8.746 & 1189495 & 46.14 \\
\hline 2 & 9.672 & 113438 & 4.40 \\
\hline 3 & 10.239 & 99806 & 3.87 \\
\hline 4 & 10.877 & 1175498 & 45.59 \\
\hline
\end{tabular}

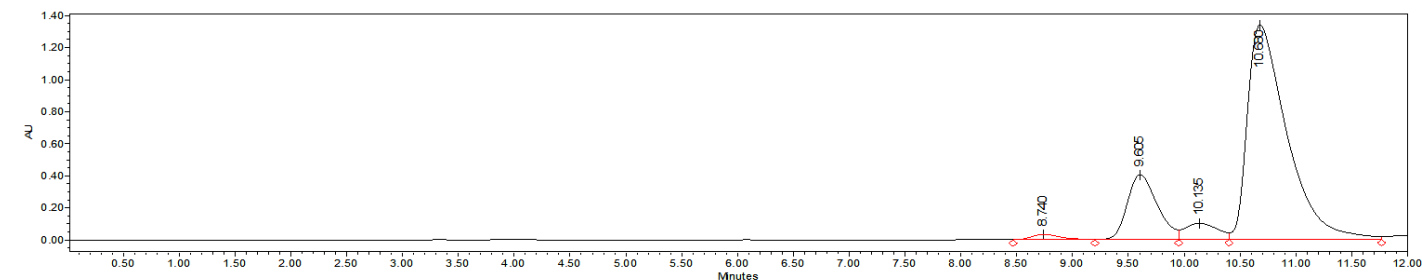

\begin{tabular}{|l|c|c|c|}
\hline & Retention Time & Area & \% Area \\
\hline 1 & 8.740 & 621084 & 1.44 \\
\hline 2 & 9.605 & 7554747 & 17.48 \\
\hline 3 & 10.135 & 2084757 & 4.82 \\
\hline 4 & 10.680 & 32965264 & 76.26 \\
\hline
\end{tabular}




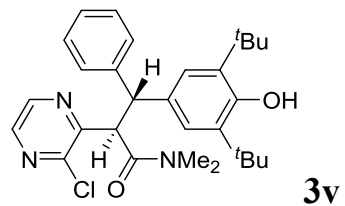

(2R,3R)-2-(3-chloropyrazin-2-yl)-3-(3,5-di-tert-butyl-4-hydroxyphenyl)- $N, N$-dimethyl-3phenylpropanamide

$0.2 \mathrm{mmol}$ scale reaction; purified by petroleum ether/ ethyl acetate $=3: 1 ; 82 \mathrm{mg}$, white solid; $83 \%$ yield, $97: 3 \mathrm{dr}, 88 \% / 50 \%$ ee; determined by HPLC analysis [Daicel chiralpak IE, $n$-hexane $/ i$-PrOH $\left.=90 / 10,1.0 \mathrm{~mL} / \mathrm{min}, \lambda=254 \mathrm{~nm}, \mathrm{t}_{1}=15.04 \mathrm{~min}, \mathrm{t}_{2}=19.69 \mathrm{~min}, \mathrm{t}_{3}=23.73 \mathrm{~min}, \mathrm{t}_{4}=26.42 \mathrm{~min}\right]$;

${ }^{1}$ H NMR $(400 \mathrm{MHz}$, Chloroform- $d) \delta 8.44(\mathrm{~d}, J=2.4 \mathrm{~Hz}, 1 \mathrm{H}), 8.05(\mathrm{~d}, J=2.4 \mathrm{~Hz}, 1 \mathrm{H}), 7.21-7.17$ $(\mathrm{m}, 2 \mathrm{H}), 7.17-7.11(\mathrm{~m}, 4 \mathrm{H}), 7.06-7.01(\mathrm{~m}, 1 \mathrm{H}), 5.43-5.40(\mathrm{~m}, 1 \mathrm{H}), 5.23-5.19(\mathrm{~m}, 1 \mathrm{H}), 5.10$ (s, 1H), $2.73(\mathrm{~d}, J=2.8 \mathrm{~Hz}, 6 \mathrm{H}), 1.38(\mathrm{~s}, 18 \mathrm{H})$;

${ }^{13}$ C NMR (101 MHz, Chloroform- $d$ ) $\delta 169.4,152.5,152.1,148.1,142.7,141.5,135.5,132.0,128.2$, 126.1, 125.2, 52.6, 49.7, 37.1, 35.9, 34.3, 30.3;

HRMS (ESI) scaled for [M+Na] $]^{+} \mathrm{C}_{29} \mathrm{H}_{36} \mathrm{ClN}_{3} \mathrm{O}_{2} \mathrm{Na}, \mathrm{m} / \mathrm{z}: 516.2388,518.2359$, observed: 516.2383, 518.2361;

$[\boldsymbol{\alpha}]_{\mathbf{D}}{ }^{\mathbf{2 0}}=222.2^{\circ}\left(c\right.$ 0.96, $\left.\mathrm{CH}_{2} \mathrm{Cl}_{2}\right)$;

m.p. $=170{ }^{\circ} \mathrm{C}$.

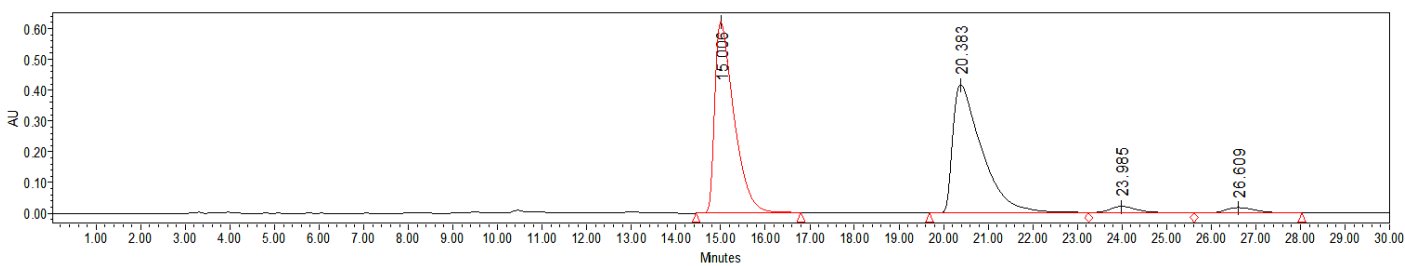

\begin{tabular}{|l|c|c|c|}
\hline & Retention Time & Area & \% Area \\
\hline 1 & 15.006 & 18519826 & 47.14 \\
\hline 2 & 20.383 & 18957990 & 48.26 \\
\hline 3 & 23.985 & 969959 & 2.47 \\
\hline 4 & 26.609 & 837137 & 2.13 \\
\hline
\end{tabular}

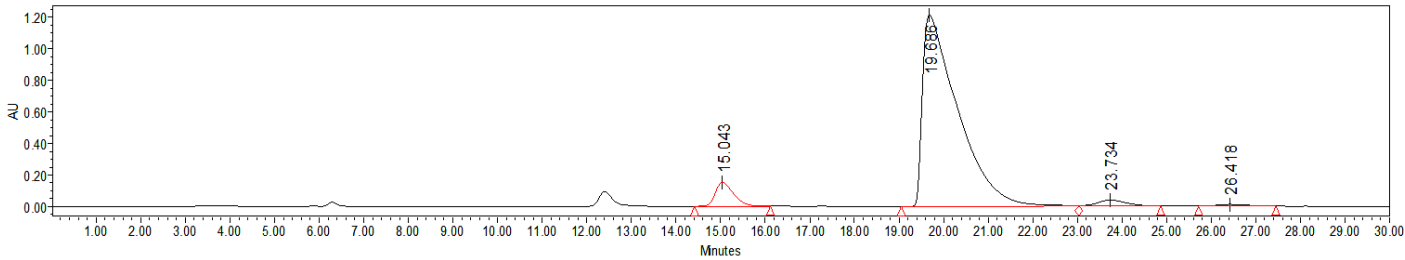

\begin{tabular}{|l|c|c|c|}
\hline & Retention Time & Area & \% Area \\
\hline 1 & 15.043 & 4287627 & 5.85 \\
\hline 2 & 19.686 & 66702207 & 91.07 \\
\hline 3 & 23.734 & 1689705 & 2.31 \\
\hline 4 & 26.418 & 563311 & 0.77 \\
\hline
\end{tabular}




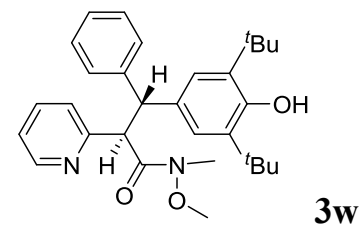

(2R,3R)-3-(3,5-di-tert-butyl-4-hydroxyphenyl)- $N$-methoxy- $N$-methyl-3-phenyl-2-(pyridin-2yl)propenamide

$0.2 \mathrm{mmol}$ scale reaction; purified by petroleum ether/ ethyl acetate $=3: 1 ; 75 \mathrm{mg}$, white solid; $78 \%$ yield, 80:20 dr, 94\%/59\% ee; determined by HPLC analysis [Daicel chiralpak IA, $n$-hexane/ $i$-PrOH $\left.=90 / 10,1.0 \mathrm{~mL} / \mathrm{min}, \lambda=254 \mathrm{~nm}, \mathrm{t}_{1}=5.07 \mathrm{~min}, \mathrm{t}_{2}=7.62 \mathrm{~min}, \mathrm{t}_{3}=9.08 \mathrm{~min}, \mathrm{t}_{4}=12.45 \mathrm{~min}\right]$;

${ }^{1} \mathbf{H}$ NMR (400 MHz, Chloroform- $d$ ) $\delta 8.48-8.31(\mathrm{~m}, 1 \mathrm{H}), 7.60-7.26(\mathrm{~m}, 3 \mathrm{H}), 7.22-7.04(\mathrm{~m}$, 5H), $7.02-6.75(\mathrm{~m}, 2 \mathrm{H}), 5.36-5.33(\mathrm{~m}, 1 \mathrm{H}), 5.04(\mathrm{~s}, 1 \mathrm{H}), 4.92-4.84(\mathrm{~m}, 1 \mathrm{H}), 3.47(\mathrm{~s}, 3 \mathrm{H}), 2.98$ (s, 3H), $1.39(\mathrm{~s}, 14 \mathrm{H}), 1.25(\mathrm{~s}, 4 \mathrm{H})$;

${ }^{13}$ C NMR (101 MHz, Chloroform- $d$ ) $\delta$ 172.8, 158.1, 152.1, 148.5, 142.1, 136.2, 135.4, 134.9, 133.2, 128.6, 128.3, 127.9, 125.7, 124.5, 123.5, 121.7, 61.6, 54.5, 54.0, 34.3, 32.1, 30.3;

IR (neat): 2952, 2360, 1647, 1550, 1499, 1458, 1354, 1232, 1197, 1109, 992, 933, 887, 812, 748, $699,643,587,540 \mathrm{~cm}^{-1}$;

HRMS (ESI) scaled for $[\mathrm{M}+\mathrm{H}]^{+}: \mathrm{C}_{30} \mathrm{H}_{39} \mathrm{~N}_{2} \mathrm{O}_{3}, \mathrm{~m} / \mathrm{z}$ : 475.2955, observed: 475.2950; $[\boldsymbol{\alpha}]_{\mathbf{D}}{ }^{\mathbf{2 0}}=-45.3^{\circ}\left(c 1.42, \mathrm{CH}_{2} \mathrm{Cl}_{2}\right)$;

m.p. $=72{ }^{\circ} \mathrm{C}$.

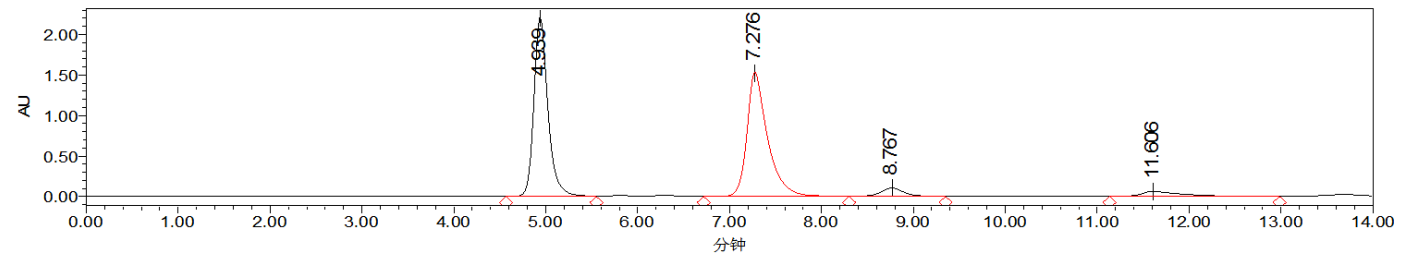

\begin{tabular}{|l|c|c|c|}
\hline & Retention Time & Area & \% Area \\
\hline 1 & 4.939 & 23525251 & 45.70 \\
\hline 2 & 7.276 & 23965468 & 46.56 \\
\hline 3 & 8.767 & 1953677 & 3.80 \\
\hline 4 & 11.606 & 2028438 & 3.94 \\
\hline
\end{tabular}

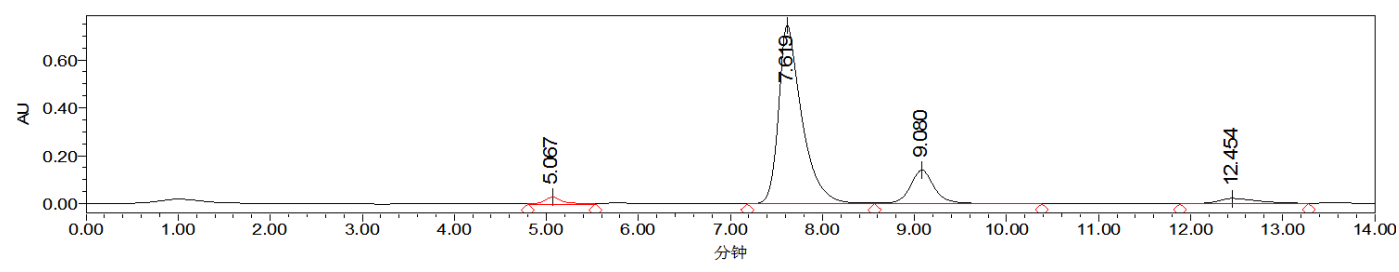

\begin{tabular}{|l|c|c|c|}
\hline & Retention Time & Area & \% Area \\
\hline 1 & 5.067 & 429141 & 2.52 \\
\hline 2 & 7.619 & 13187410 & 77.54 \\
\hline 3 & 9.080 & 2688215 & 15.81 \\
\hline 4 & 12.454 & 701659 & 4.13 \\
\hline
\end{tabular}




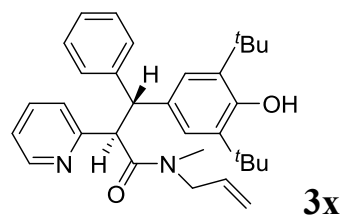

(2R,3R)- $N$-allyl-3-(3,5-di-tert-butyl-4-hydroxyphenyl)- $N$-methyl-3-phenyl-2-(pyridin-2yl)propenamide

$0.2 \mathrm{mmol}$ scale reaction; purified by petroleum ether/ ethyl acetate $=3: 1 ; 62 \mathrm{mg}$, white solid; $64 \%$ yield, $73: 27 \mathrm{dr}, 88 \% / 55 \%$ ee; determined by HPLC analysis [Daicel chiralpak IC, $n$-hexane $/ i$-PrOH $\left.=97 / 3,1.0 \mathrm{~mL} / \mathrm{min}, \lambda=254 \mathrm{~nm}, \mathrm{t}_{1}=6.88 \mathrm{~min}, \mathrm{t}_{2}=7.85 \mathrm{~min}, \mathrm{t}_{3}=9.82 \mathrm{~min}, \mathrm{t}_{4}=13.72 \mathrm{~min}\right]$;

${ }^{1} \mathbf{H}$ NMR (400 MHz, Chloroform- $d$ ) $\delta 8.38-8.29(\mathrm{~m}, 1 \mathrm{H}), 7.62-7.27(\mathrm{~m}, 3 \mathrm{H}), 7.26-7.01(\mathrm{~m}$, $5 \mathrm{H}), 7.00-6.80(\mathrm{~m}, 2 \mathrm{H}), 5.55-5.17(\mathrm{~m}, 1 \mathrm{H}), 5.17-4.83(\mathrm{~m}, 4 \mathrm{H}), 4.82-4.52(\mathrm{~m}, 1 \mathrm{H}), 4.50-3.98$ (m, 1H), $3.73-3.33(\mathrm{~m}, 1 \mathrm{H}), 3.04-2.98(\mathrm{~m}, 2 \mathrm{H}), 2.72-2.71(\mathrm{~m}, 1 \mathrm{H}), 1.39-1.38(\mathrm{~m}, 12 \mathrm{H}), 1.26$ $-1.25(\mathrm{~m}, 6 \mathrm{H})$;

${ }^{13}$ C NMR (101 MHz, Chloroform- $d$ ) $\delta$ 171.6, 171.4, 158.8, 158.4, 152.3, 151.6, 148.2, 142.2, 136.4, $135.5,133.4,132.5,129.2,128.5,128.0,125.7,124.4,123.2,121.8,116.7,116.3,56.8,56.0,54.4$, 53.8, 50.2, 35.3, 34.3, 34.1, 30.3;

IR (neat): 2953, 2361, 1636, 1590, 1469, 1432, 1399, 1361, 1232, 1149, 1113, 990, 920, 749, 698, $640,578,540 \mathrm{~cm}^{-1}$;

HRMS (ESI) scaled for $[\mathrm{M}+\mathrm{H}]^{+}: \mathrm{C}_{32} \mathrm{H}_{41} \mathrm{~N}_{2} \mathrm{O}_{2}, \mathrm{~m} / \mathrm{z}$ : 485.3163, observed: 485.3158;

$[\boldsymbol{\alpha}]_{\mathbf{D}}{ }^{20}=25.9^{\circ}\left(c 1.02, \mathrm{CH}_{2} \mathrm{Cl}_{2}\right)$;

m.p. $=80^{\circ} \mathrm{C}$.

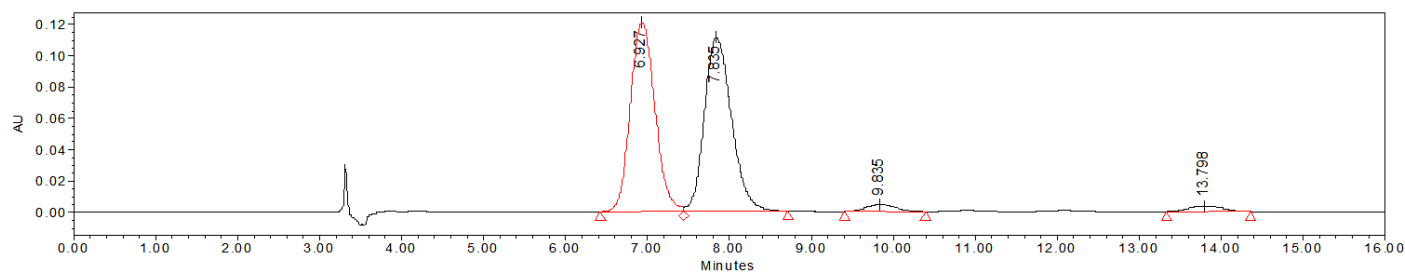

\begin{tabular}{|l|c|c|c|}
\hline & Retention Time & Area & $\%$ Area \\
\hline 1 & 6.927 & 2546342 & 47.53 \\
\hline 2 & 7.835 & 2574999 & 48.06 \\
\hline 3 & 9.835 & 124886 & 2.33 \\
\hline 4 & 13.798 & 111548 & 2.08 \\
\hline
\end{tabular}

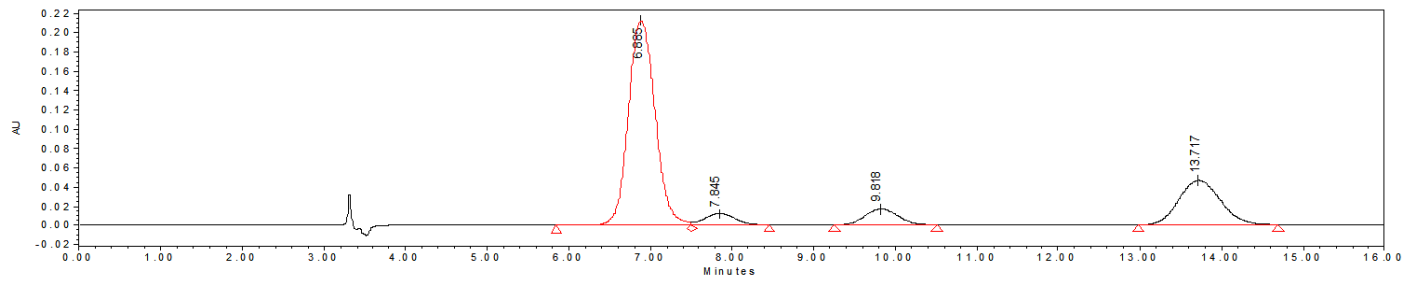

\begin{tabular}{|l|c|c|c|}
\hline & Retention Time & Area & \% Area \\
\hline 1 & 6.885 & 4831046 & 66.41 \\
\hline 2 & 7.845 & 309052 & 4.25 \\
\hline 3 & 9.818 & 478096 & 6.57 \\
\hline 4 & 13.717 & 1656711 & 22.77 \\
\hline
\end{tabular}




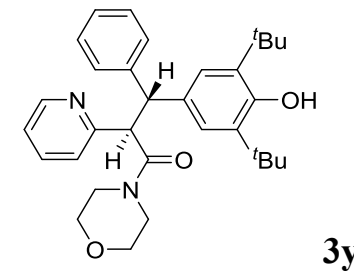

(2R,3R)-3-(3,5-di-tert-butyl-4-hydroxyphenyl)-1-morpholino-3-phenyl-2-(pyridin-2-

\section{yl)propan-1-one}

$0.2 \mathrm{mmol}$ scale reaction; purified by petroleum ether/ ethyl acetate $=3: 1 ; 64.5 \mathrm{mg}$, white solid; $65 \%$ yield, $68: 32 \mathrm{dr}, 88 \% / 53 \%$ ee; determined by HPLC analysis [Daicel chiralpak ID, $n$-hexane/i-PrOH $\left.=80 / 20,1.0 \mathrm{~mL} / \mathrm{min}, \lambda=254 \mathrm{~nm}, \mathrm{t}_{1}=6.65 \mathrm{~min}, \mathrm{t}_{2}=8.05 \mathrm{~min}, \mathrm{t}_{3}=16.24 \mathrm{~min}, \mathrm{t}_{4}=22.04 \mathrm{~min}\right] ;$

${ }^{1}$ H NMR (400 MHz, Chloroform- $d$ ) $\delta 8.35-8.30(\mathrm{~m}, 1 \mathrm{H}), 7.55-7.40(\mathrm{~m}, 2 \mathrm{H}), 7.39-7.26(\mathrm{~m}$, 2H), $7.21-7.14(\mathrm{~m}, 3 \mathrm{H}), 7.10-7.05(\mathrm{~m}, 1 \mathrm{H}), 7.02-6.81(\mathrm{~m}, 2 \mathrm{H}), 5.08(\mathrm{~s}, 1 \mathrm{H}), 4.97-4.86(\mathrm{~m}$, $2 \mathrm{H}), 3.75-3.38(\mathrm{~m}, 5 \mathrm{H}), 3.37-3.02(\mathrm{~m}, 3 \mathrm{H}), 1.39(\mathrm{~s}, 12 \mathrm{H}), 1.25(\mathrm{~s}, 6 \mathrm{H})$;

${ }^{13}$ C NMR (101 MHz, Chloroform- $d$ ) $\delta 170.4,158.3,152.3,148.2,141.9,136.4,135.7,135.0,133.3$, 128.6, 127.9, 125.8, 125.4, 124.5, 123.4, 121.8, 66.6, 55.6, 53.8, 46.4, 42.4, 34.3, 30.3;

IR (neat): 2956, 1631, 1430, 1229, 1112, 1029, 746, $699 \mathrm{~cm}^{-1}$;

HRMS (ESI) scaled for $[\mathrm{M}+\mathrm{H}]^{+}: \mathrm{C}_{32} \mathrm{H}_{41} \mathrm{~N}_{2} \mathrm{O}_{3}, \mathrm{~m} / \mathrm{z}$ : 501.3112, observed: 501.3108;

$[\boldsymbol{\alpha}]_{\mathbf{D}}{ }^{\mathbf{2 0}}=8.1^{\circ}\left(c 0.12, \mathrm{CH}_{2} \mathrm{Cl}_{2}\right)$;

m.p. $=80^{\circ} \mathrm{C}$.

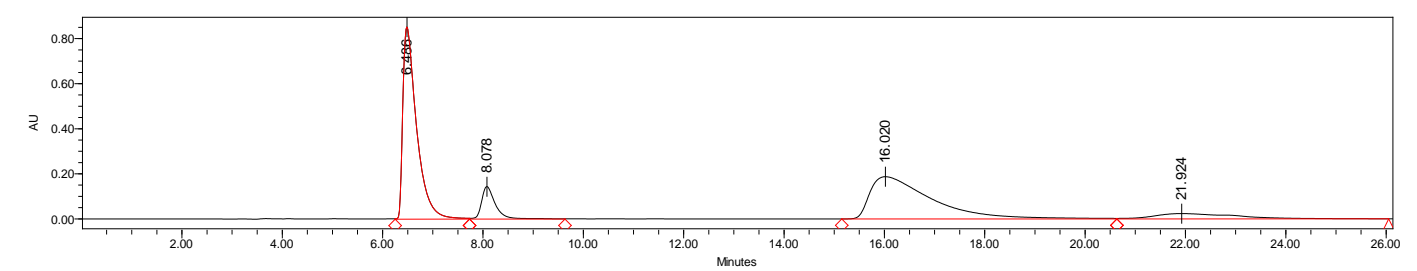

\begin{tabular}{|l|c|c|c|}
\hline & Retention Time & Area & \% Area \\
\hline 1 & 6.486 & 16252962 & 43.09 \\
\hline 2 & 8.078 & 2798940 & 7.42 \\
\hline 3 & 16.020 & 15990698 & 42.39 \\
\hline 4 & 21.924 & 2676279 & 7.10 \\
\hline
\end{tabular}

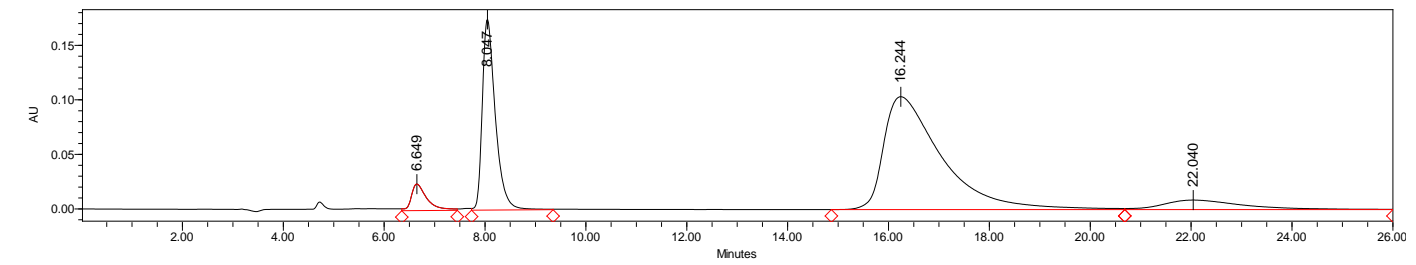

\begin{tabular}{|l|c|c|c|}
\hline & Retention Time & Area & \% Area \\
\hline 1 & 6.649 & 526108 & 4.07 \\
\hline 2 & 8.047 & 3158589 & 24.43 \\
\hline 3 & 16.244 & 8263784 & 63.92 \\
\hline 4 & 22.040 & 980735 & 7.59 \\
\hline
\end{tabular}




\section{Experimental procedure for the scale-up reaction}

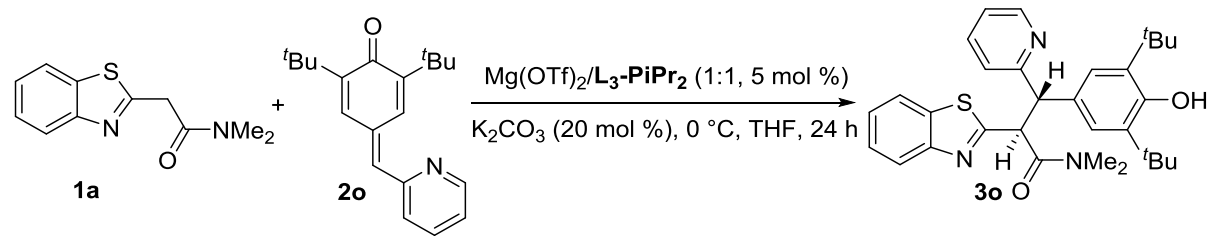

$\mathrm{Mg}(\mathrm{OTf})_{2}(0.1 \mathrm{mmol}, 32.2 \mathrm{mg}), N, N^{\prime}$-dioxide ligand $\mathbf{L}_{3}-\mathbf{P i P r}_{2}(0.1 \mathrm{mmol}, 64.8 \mathrm{mg})$ and $\mathrm{K}_{2} \mathrm{CO}_{3}$ (20 mol\%, $0.4 \mathrm{mmol}, 55.3 \mathrm{mg}), \alpha$-Azaarylamide $1 \mathrm{a}(2.0 \mathrm{mmol}, 440 \mathrm{mg})$ were weighted into a 50 $\mathrm{mL}$ round-bottomed flask. Then $15 \mathrm{~mL}$ of anhydrous tetrahydrofuran was added. The mixture was stirred at $35{ }^{\circ} \mathrm{C}$ for 45 minutes. After stirring at $0{ }^{\circ} \mathrm{C}$ for a few minutes, quinone methide 20 (2.0 mmol, $590 \mathrm{mg}$ ) was added into the flask. The mixture was stirred at $0{ }^{\circ} \mathrm{C}$ for $12 \mathrm{~h}$. The reaction mixture was detected by TLC (petroleum ether/ethyl acetate $=3: 1$ ). After completion, the product was purified by flash chromatography on silica gel (petroleum ether/ethyl acetate $=3: 1$ ) to afford the desired product 3o (1.028g, 99\% yield with 98:2 dr and 93\% ee).

\section{Experimental procedure for the synthesis of compounds $4 \mathrm{~h}$ and $5 \mathrm{~h}$}

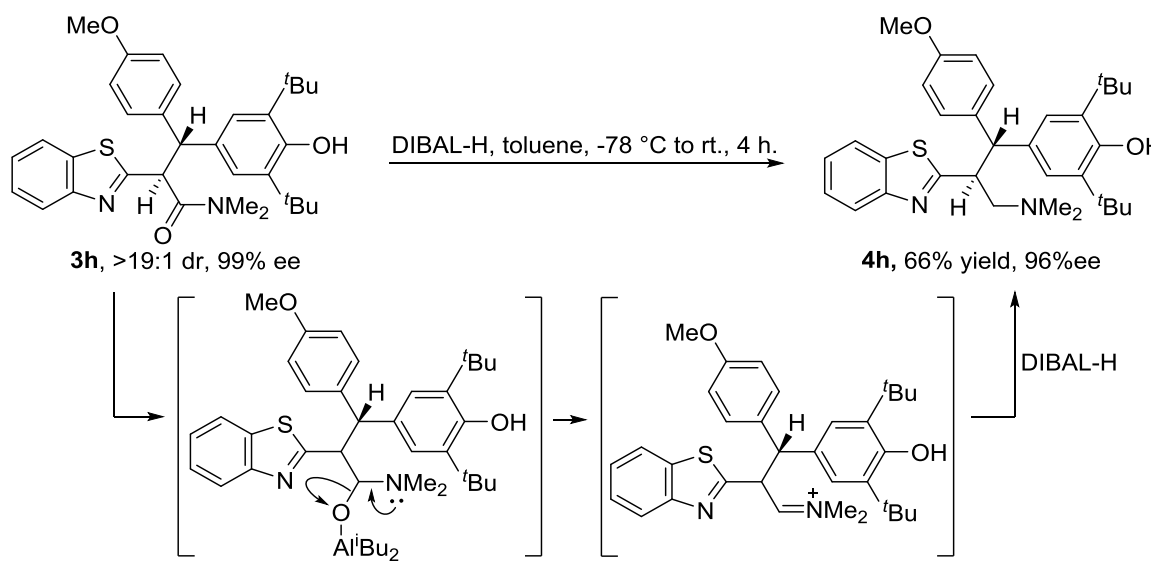

Added the substrate $\mathbf{3 h}(0.20 \mathrm{mmol}, 108.8 \mathrm{mg})$ to an oven-dried reaction tube then make sure it filled with nitrogen. Next, added $4 \mathrm{~mL}$ toluene to dissolve the substrate and cooled it to $-78{ }^{\circ} \mathrm{C}$. Finally, after added DIBAL-H (1.5 M in toluene, 3.0 equiv., $0.4 \mathrm{~mL})$ slowly, 4 hours later, heated the temperature to room temperature. The reaction was quenched by a saturated aqueous solution of seignette salt and extracted with ethyl acetate. The organic phase was detected by TLC (petroleum ether/ethyl acetate $=1: 1)$. After completion, the product was purified by flash chromatography on silica gel (petroleum ether/ethyl acetate $=1: 1)$ to afford the desired product $4 \mathrm{~h}(69.8 \mathrm{mg}, 66 \%$ yield $96 \%$ ee).

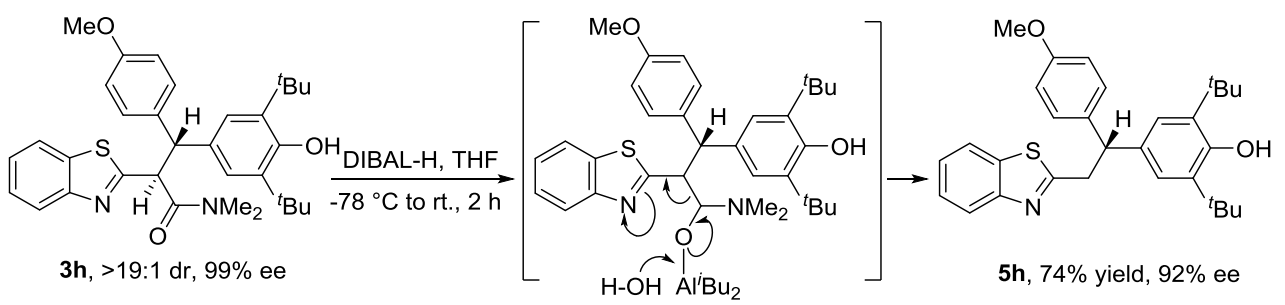

Added the substrate $3 \mathbf{h}(0.20 \mathrm{mmol}, 108.8 \mathrm{mg})$ to an oven-dried reaction tube then make sure it filled with nitrogen. Next, added $4 \mathrm{~mL}$ THF to dissolve the substrate and cooled it to $-78^{\circ} \mathrm{C}$. Finally, 
after added DIBAL-H (1.5 M in toluene, 3.0 equiv., $0.4 \mathrm{~mL})$ slowly, 2 hours later, heated the temperature to room temperature. The reaction was quenched by a saturated aqueous solution of seignette salt and extracted with ethyl acetate. The organic phase was detected by TLC (petroleum ether/ethyl acetate $=10: 1)$. After completion, the product was purified by flash chromatography on silica gel (petroleum ether/ethyl acetate $=10: 1)$ to afford the desired product $5 \mathbf{h}(70.0 \mathrm{mg}, 74 \%$ yield $92 \%$ ee).

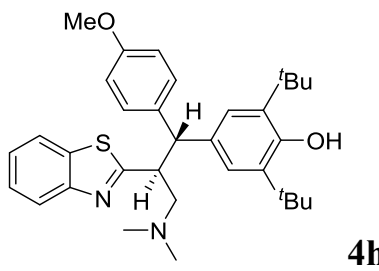

4-((1R,2S)-2-(benzo[d]thiazol-2-yl)-3-(dimethylamino)-1-(4-methoxyphenyl)propyl)-2,6-ditert-butylphenol

$0.2 \mathrm{mmol}$ scale reaction; purified by petroleum ether/ethyl acetate $=1: 1 ; 70.0 \mathrm{mg}$, white solid; $66 \%$ yield, 96\% ee; determined by HPLC analysis [Daicel chiralpak IG, $n$-hexane $/ i-\mathrm{PrOH}=95 / 5,0.5$ $\left.\mathrm{mL} / \mathrm{min}, \lambda=254 \mathrm{~nm}, \mathrm{t}_{1}=8.77 \mathrm{~min}, \mathrm{t}_{2}=9.55 \mathrm{~min}\right]$;

${ }^{1}$ H NMR (400 MHz, Chloroform- $d$ ) $\delta 7.91-7.88(\mathrm{~m}, 1 \mathrm{H}), 7.72-7.66(\mathrm{~m}, 1 \mathrm{H}), 7.36-7.32(\mathrm{~m}$, $1 \mathrm{H}), 7.24-7.13(\mathrm{~m}, 5 \mathrm{H}), 6.64-6.54(\mathrm{~m}, 2 \mathrm{H}), 5.07(\mathrm{~s}, 1 \mathrm{H}), 4.27-4.13(\mathrm{~m}, 2 \mathrm{H}), 3.59(\mathrm{~s}, 3 \mathrm{H})$, $2.97-2.92(\mathrm{~m}, 1 \mathrm{H}), 2.54-2.40(\mathrm{~m}, 1 \mathrm{H}), 2.14(\mathrm{~s}, 6 \mathrm{H}), 1.43(\mathrm{~s}, 18 \mathrm{H})$;

${ }^{13}$ C NMR (101 MHz, Chloroform- $d$ ) $\delta$ 174.7, 157.5, 152.7, 152.3, 135.9, 135.1, 134.8, 133.4, 128.8, 125.4, 124.2, 124.1, 122.5, 121.4, 113.5, 64.1, 55.5, 54.9, 48.4, 45.7, 34.3, 30.3;

IR (neat): 3627, 2951, 2818, 2764, 1610, 1509, 1455, 1246, 1178, 1034, 835, 758, 641, $536 \mathrm{~cm}^{-1}$; HRMS (ESI) scaled for $[\mathrm{M}+\mathrm{H}]^{+}: \mathrm{C}_{33} \mathrm{H}_{43} \mathrm{~N}_{2} \mathrm{O}_{2} \mathrm{~S}, \mathrm{~m} / \mathrm{z}$ : 531.3040, observed: 531.3043; $[\boldsymbol{\alpha}]_{\mathbf{D}}{ }^{\mathbf{2 0}}=-24.3^{\circ}\left(c 1.40, \mathrm{CH}_{2} \mathrm{Cl}_{2}\right)$; m.p. $=90^{\circ} \mathrm{C}$.

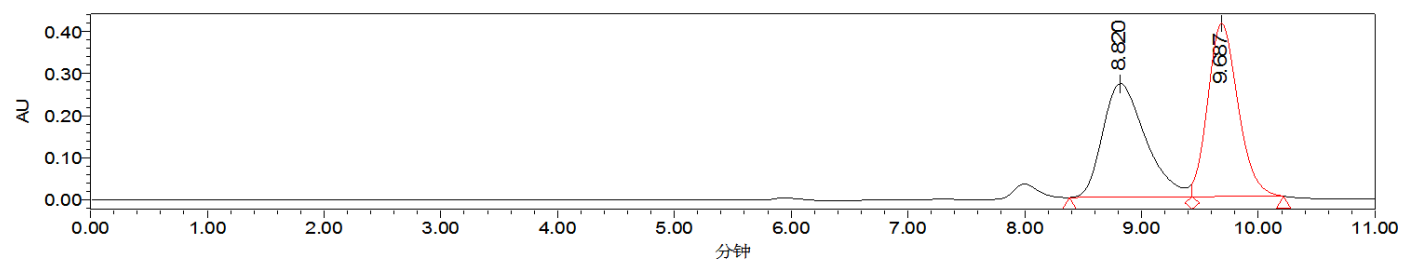

\begin{tabular}{|l|c|c|c|}
\hline & Retention Time & Area & \% Area \\
\hline 1 & 8.820 & 6921875 & 48.36 \\
\hline 2 & 9.687 & 7391802 & 51.64 \\
\hline
\end{tabular}

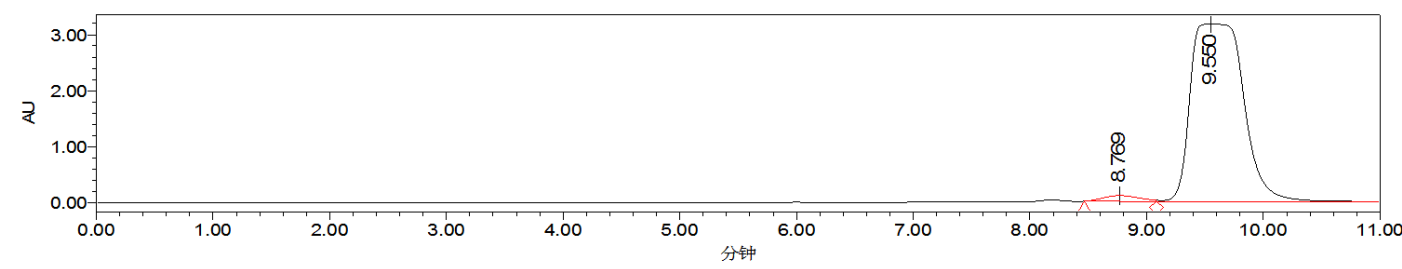

\begin{tabular}{|c|c|c|c|}
\hline & Retention Time & Area & \% Area \\
\hline 1 & 8.769 & 2202352 & 2.16 \\
\hline 2 & 9.550 & 99768633 & 97.84 \\
\hline
\end{tabular}




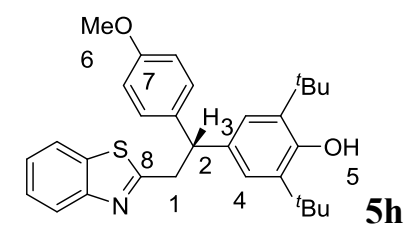

(R)-4-(2-(benzo[d]thiazol-2-yl)-1-(4-methoxyphenyl)ethyl)-2,6-di-tert-butylphenol

$0.2 \mathrm{mmol}$ scale reaction; purified by petroleum ether/ethyl acetate $=10: 1 ; 70.0 \mathrm{mg}$, white solid; $74 \%$ yield, $92 \%$ ee; determined by HPLC analysis [Daicel chiralpak IG, $n$-hexane/ $i$-PrOH $=49 / 1,0.5$ $\left.\mathrm{mL} / \mathrm{min}, \lambda=254 \mathrm{~nm}, \mathrm{t}_{1}=19.97 \mathrm{~min}, \mathrm{t}_{2}=21.58 \mathrm{~min}\right]$;

${ }^{1} \mathbf{H}$ NMR (400 MHz, Chloroform- $\left.d\right) \delta 7.95-7.93(\mathrm{~m}, 1 \mathrm{H}), 7.74-7.71(\mathrm{~m}, 1 \mathrm{H}), 7.42-7.38(\mathrm{~m}$, $1 \mathrm{H}), 7.30-7.22(\mathrm{~m}, 3 \mathrm{H}), 7.05\left(\mathrm{C}_{3}-\mathrm{H}, \mathrm{s}, 2 \mathrm{H}\right), 6.83-6.78(\mathrm{~m}, 2 \mathrm{H}), 5.06(-\mathrm{OH}, \mathrm{s}, 1 \mathrm{H}), 4.54-4.50$ $\left(\mathrm{C}_{2}-\mathrm{H}, \mathrm{m}, 1 \mathrm{H}\right), 3.85-3.77\left(\mathrm{C}_{1}-\mathrm{H}, \mathrm{m}, 2 \mathrm{H}\right), 3.76\left(\mathrm{C}_{6}-\mathrm{H}, \mathrm{s}, 3 \mathrm{H}\right), 1.36(\mathrm{~s}, 18 \mathrm{H})$;

${ }^{13}$ C NMR (101 MHz, Chloroform- $\left.d\right) \delta 170.5\left(\mathrm{C}_{8}\right), 158.0\left(\mathrm{C}_{7}\right), 152.9,152.2,135.7,135.5,135.2$

$\left(\mathrm{C}_{3}\right), 134.2,128.9,125.7,124.5,124.2\left(\mathrm{C}_{4}\right), 122.4,121.3,113.8,55.1\left(\mathrm{C}_{6}\right), 50.4\left(\mathrm{C}_{2}\right), 41.3\left(\mathrm{C}_{1}\right)$, 34.3, 30.2;

IR (neat): 3631, 2954, 1610, 1509, 1456, 1434, 1390, 1360, 1303, 1247, 1178, 1035, $759 \mathrm{~cm}^{-1}$;

HRMS (ESI) scaled for [M-H]: $\mathrm{C}_{30} \mathrm{H}_{34} \mathrm{NO}_{2} \mathrm{~S}, \mathrm{~m} / \mathrm{z}: 472.2305$, observed: 472.2313;

$[\boldsymbol{\alpha}]_{\mathbf{4 3 6}}{ }^{\mathbf{2 0}}=-17.7^{\circ}\left(c 0.26, \mathrm{CH}_{2} \mathrm{Cl}_{2}\right)$;

m.p. $=70^{\circ} \mathrm{C}$.

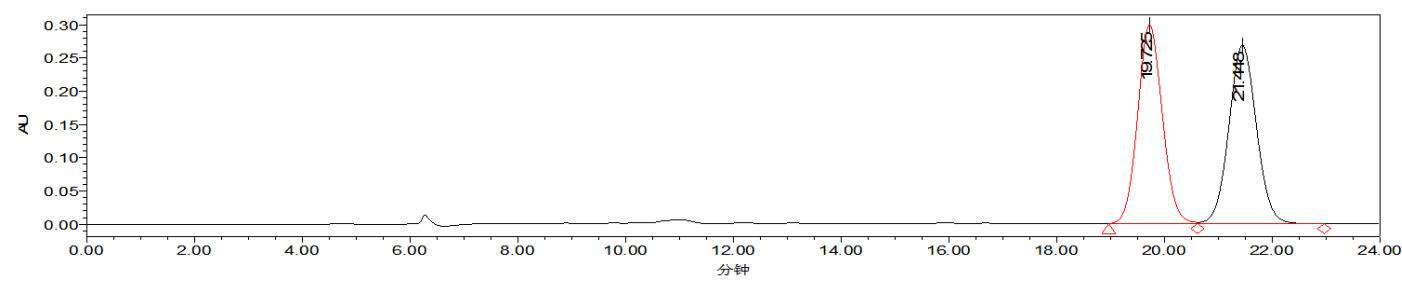

\begin{tabular}{|l|c|c|c|}
\hline & Retention Time & Area & \% Area \\
\hline 1 & 19.725 & 9538678 & 49.95 \\
\hline 2 & 21.448 & 9558184 & 50.05 \\
\hline
\end{tabular}

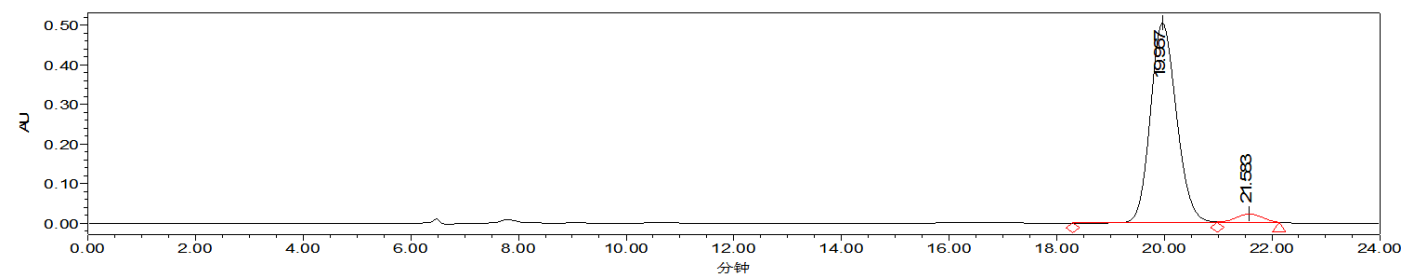

\begin{tabular}{|l|c|c|c|}
\hline & Retention Time & Area & \% Area \\
\hline 1 & 19.967 & 16969027 & 95.98 \\
\hline 2 & 21.583 & 710660 & 4.02 \\
\hline
\end{tabular}




\section{Other reactions with no products}

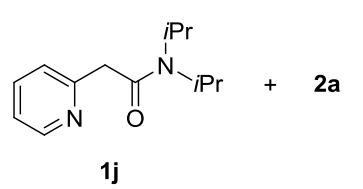

$$
\begin{gathered}
\underset{\mathrm{Mg}(\mathrm{OTf})_{2} / \mathrm{L}_{3}-\mathrm{PiPr}_{2}}{(1: 1,10 \mathrm{~mol} \%)} \\
\underset{\substack{\mathrm{K}_{2} \mathrm{CO}_{3}(50 \mathrm{~mol} \%) \\
35^{\circ} \mathrm{C}, \mathrm{THF}}}{\longrightarrow}
\end{gathered}
$$

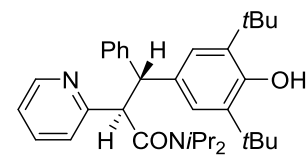

no reaction<smiles>O=C(Cc1ccccn1)N(Cc1ccccc1)c1ccccc1</smiles>
$\mathrm{Mg}(\mathrm{OTf})_{2} / \mathrm{L}_{3}-\mathrm{PiPr}_{2}$
$(1: 1,10 \mathrm{~mol} \mathrm{\%})$<smiles>CC(C)(C)c1cc(C(C(=O)O)(c2ccccc2)c2ccccn2)cc(C(C)(C)C)c1O</smiles>

no reaction

$1 \mathrm{k}$<smiles>O=C(Cc1nc2ccccc2s1)N[13C](Br)c1ccccc1</smiles>
11

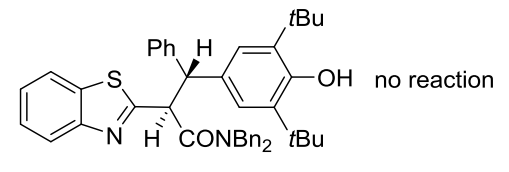

\section{Demonstration of reaction mechanism by HRMS and control experiment}

1 a (22.0 mg, $0.10 \mathrm{mmol}$ ), $\mathrm{Mg}(\mathrm{OTf})_{2}$ (32 mg, $0.10 \mathrm{~mol}$ ), $N, N^{\prime}$-dioxide ligand $\mathbf{L}_{\mathbf{3}}-\mathbf{P i P r}_{\mathbf{2}}(65 \mathrm{mg}, 0.10$ mol) were stirred in THF $(1.0 \mathrm{~mL})$ at $35{ }^{\circ} \mathrm{C}$ for $1.0 \mathrm{~h}$. Then, the mixture was analyzed by HRMS directly. $\left[\mathrm{Mg}^{2+}+\mathrm{OTf}^{-}+\mathbf{L}_{3}-\mathrm{PiPr}_{2}+\mathbf{1 a}\right]^{+}$(scaled for $\mathrm{C}_{51} \mathrm{H}_{72} \mathrm{~F}_{3} \mathrm{MgN}_{6} \mathrm{O}_{8} \mathrm{~S}_{2}{ }^{+}, \mathrm{m} / \mathrm{z}: 1041.4650$, found: 1041.4647) was detected.

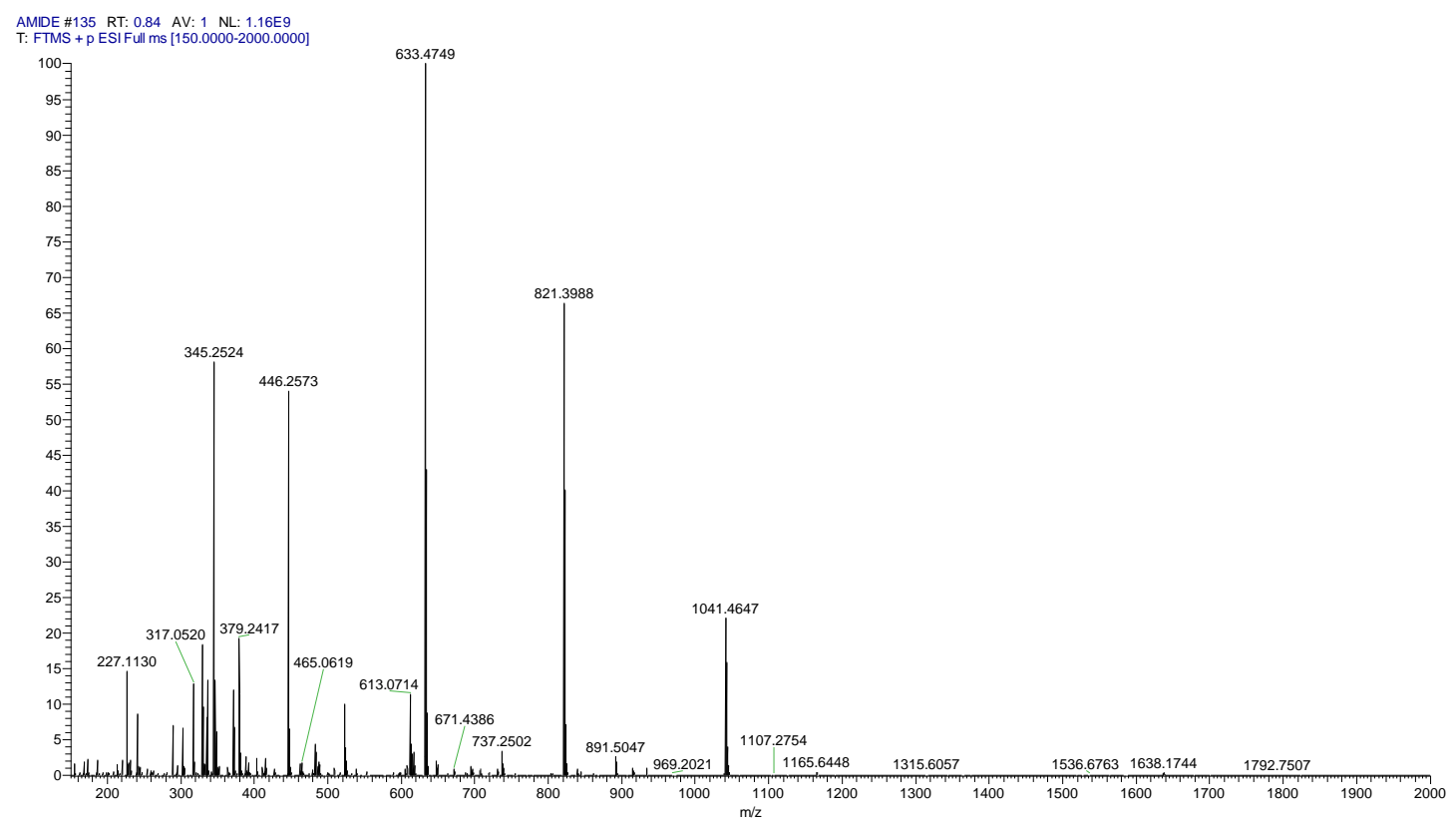

Control experiments were conducted under standard conditions: 


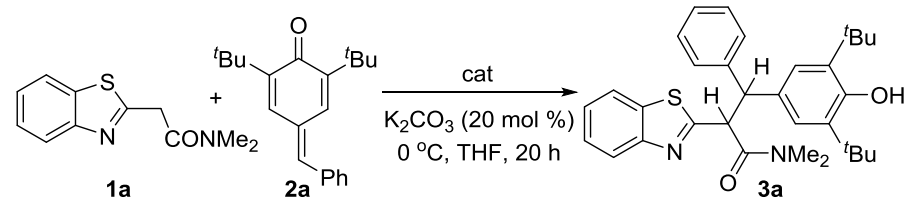

(a) no catalyst $\quad 99 \%$ yield, $1: 1 \mathrm{dr}$, racemic

(b) cat $=\mathrm{Mg}(\mathrm{OTf})_{2} \quad$ No reaction

(c) cat $=\mathrm{Mg}(\mathrm{OTf})_{2} / \mathrm{L}_{3}-\mathrm{PiPr}_{2} \quad 99 \%$ yield, $93: 7 \mathrm{dr}, 99 \% / 32 \%$ ee

\section{X-ray structure of 3a}

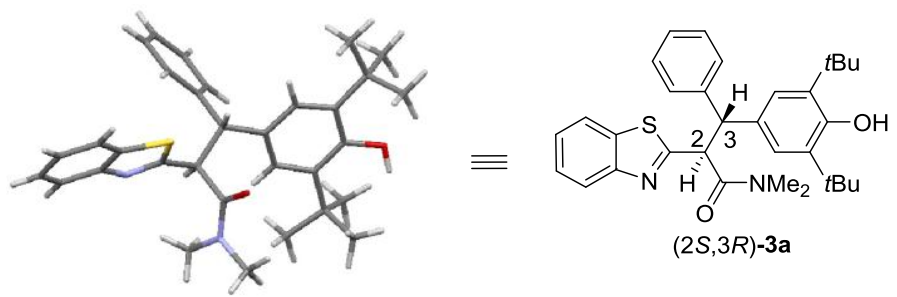

Single crystal of 3a was obtained from the mixed solvents of petroleum ether and ethyl acetate. The absolute configuration was shown above. CCDC 1878495 (3a) was assigned to be $(2 S, 3 R)$ respectively. These data can be obtained free from the Cambridge Crystallographic Data Centre via www.ccdc.cam.ac.uk/data requst/cif.

\section{Reference}

[1] Fallan, C.; Lam, H. W. Chem. Eur. J. 2012, 18, 11214-11218.

[2] Jiang, X.; Boehm, P.; Hartwig, J. F. J. Am. Chem. Soc. 2018, 140, 1239-1242.

[3] Roiser, L.; Waser, M. Org. Lett. 2017, 19, 2338-2341.

[4] Guan, X. Y.; Zhang, L. D.; You, P. S.; Liu, S. S.; Liu, Z. Q. Tetrahedron Lett. 2019, 60, 244-247.

[5] Ge, L.; Lu, X.; Cheng, C.; Chen, J.; Cao, W.; Wu, X.; Zhao, G. J. Org. Chem. 2016, 81, 9315-9325.

[6] Molleti, N.; Kang, Y. Org. Lett. 2017, 19, 958-961.

[7] Gao, S.; Xu, X. Y.; Yuan, Z. B.; Zhou, H. P.; Yao, H. Q.; Lin, A. J. Eur. J. Org. Chem. 2016, 3006-3012. 


\section{Copies of CD spectra for the products in $\mathrm{MeOH}$}
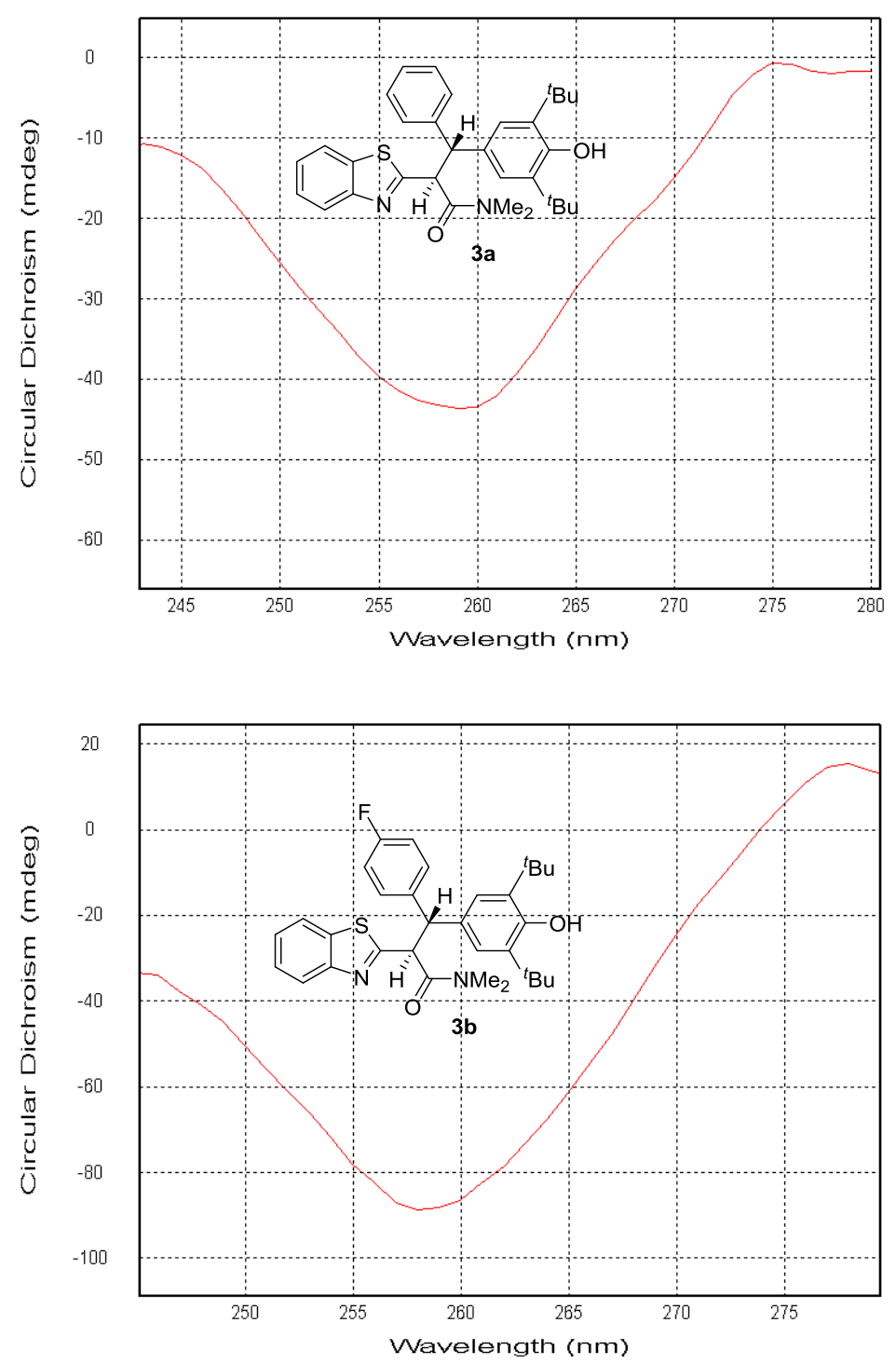

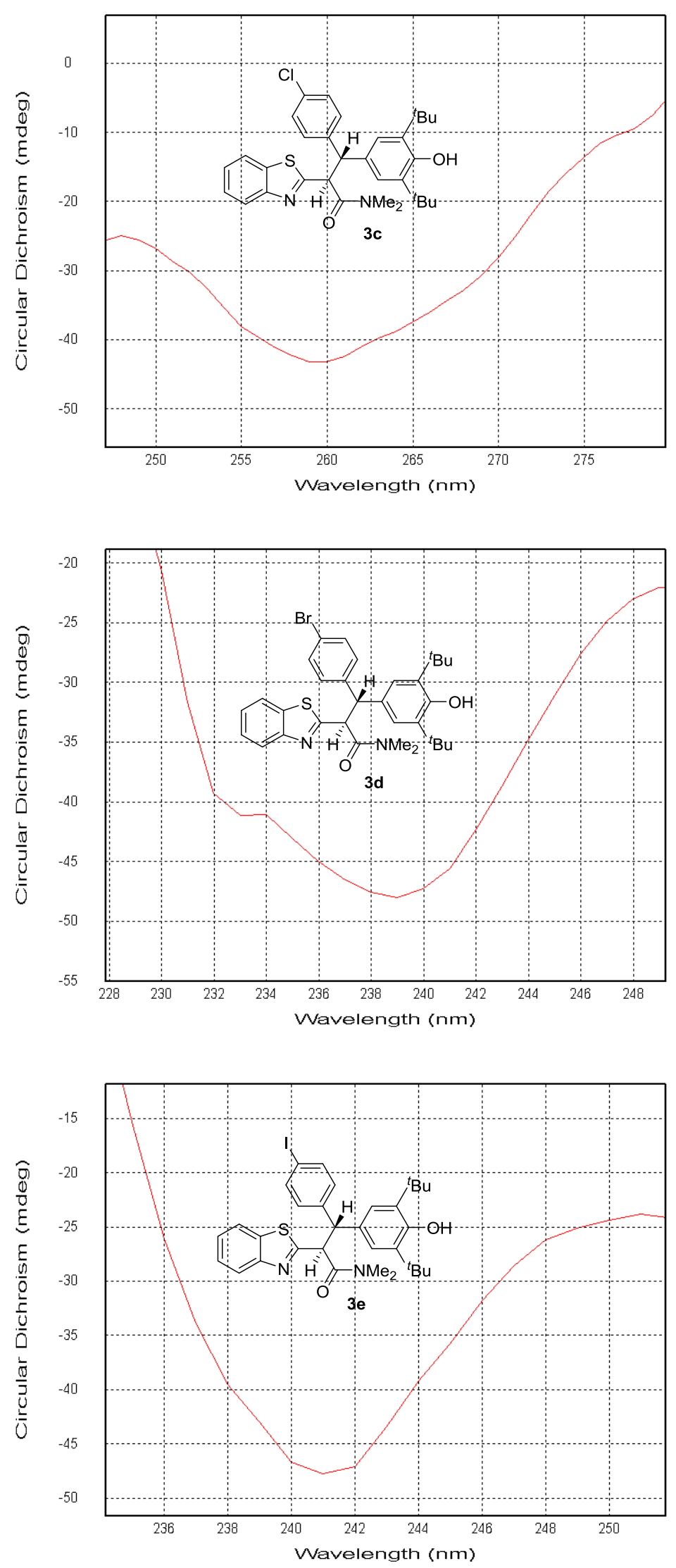

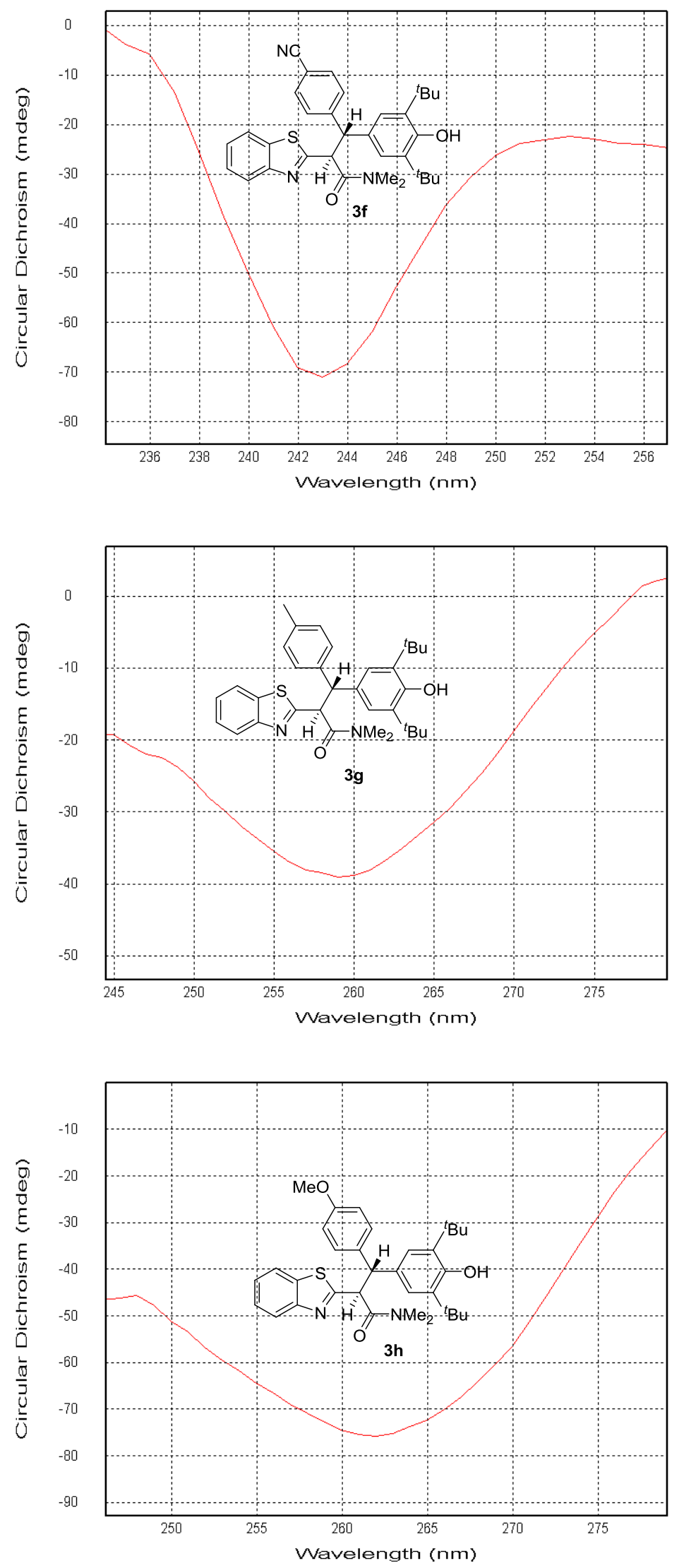

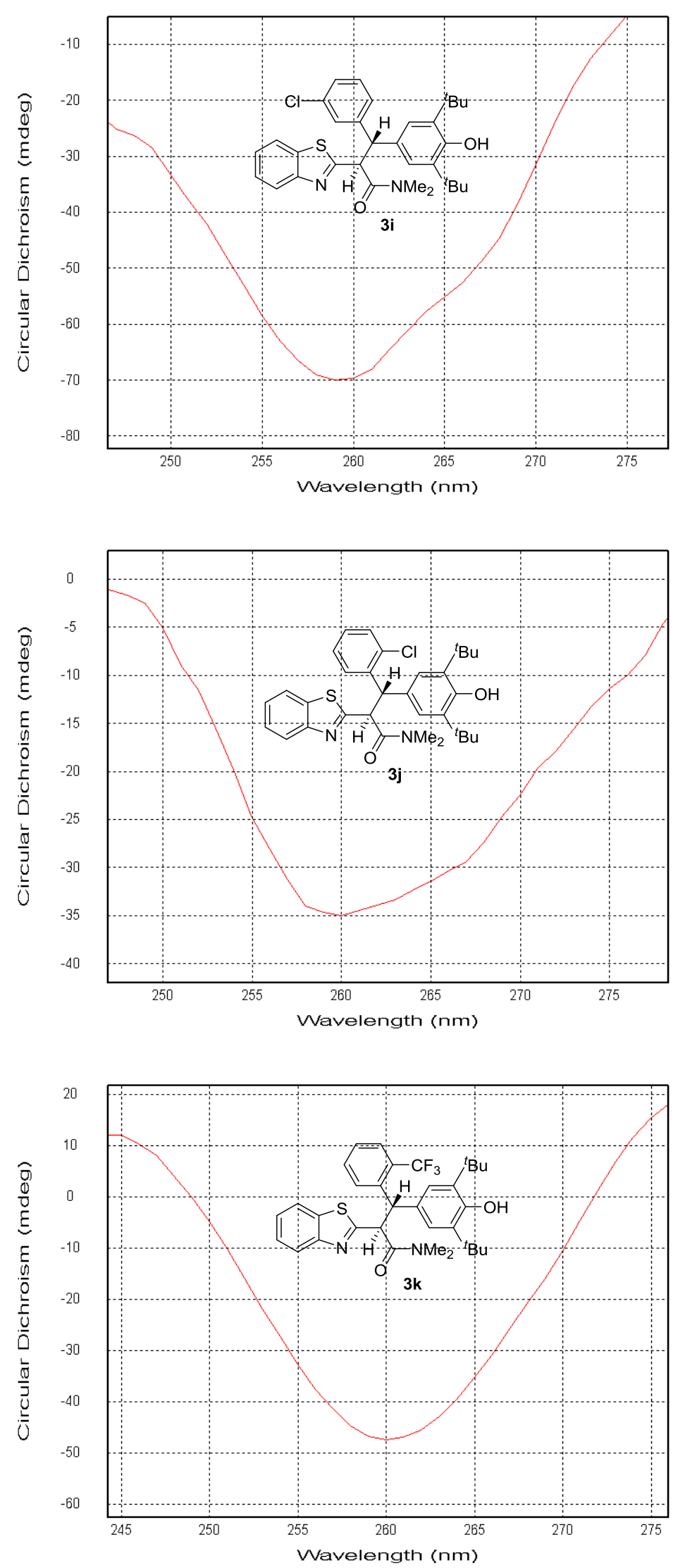

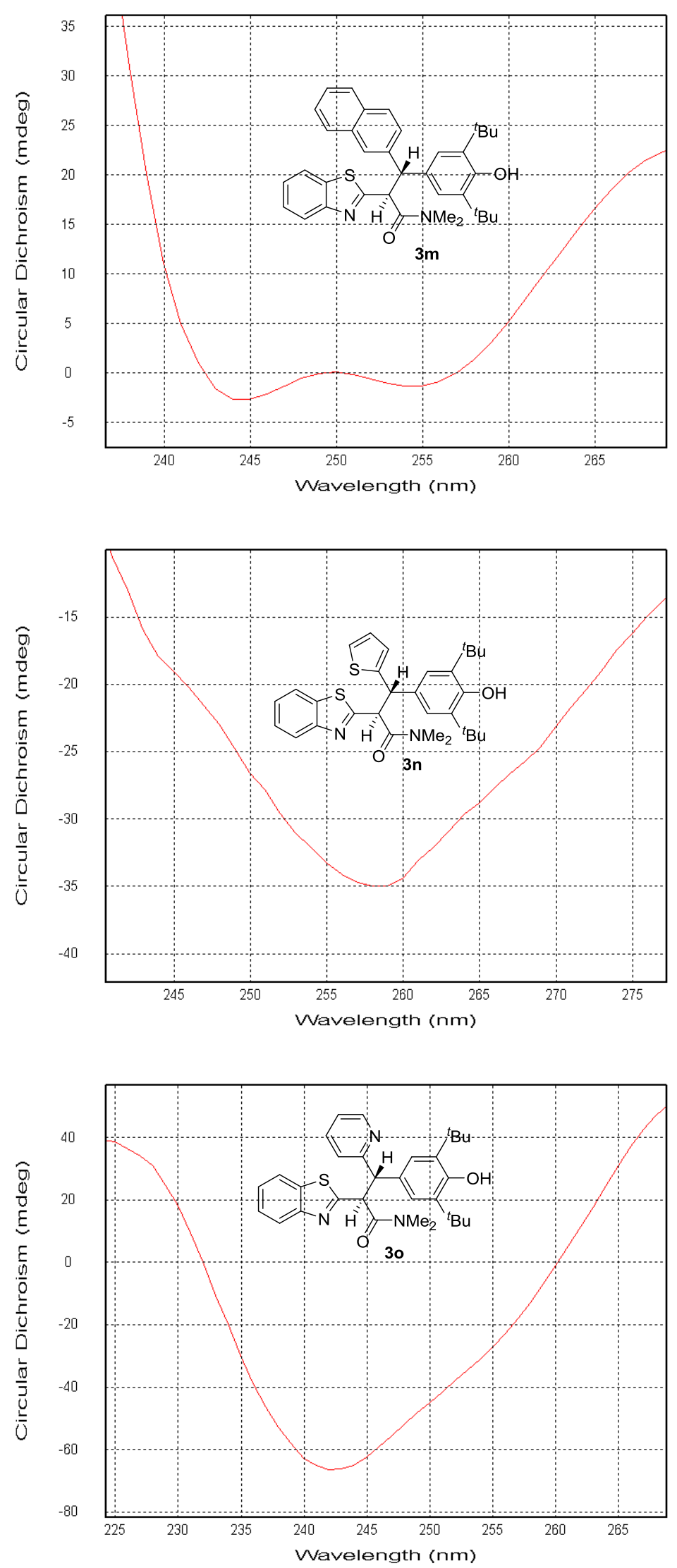


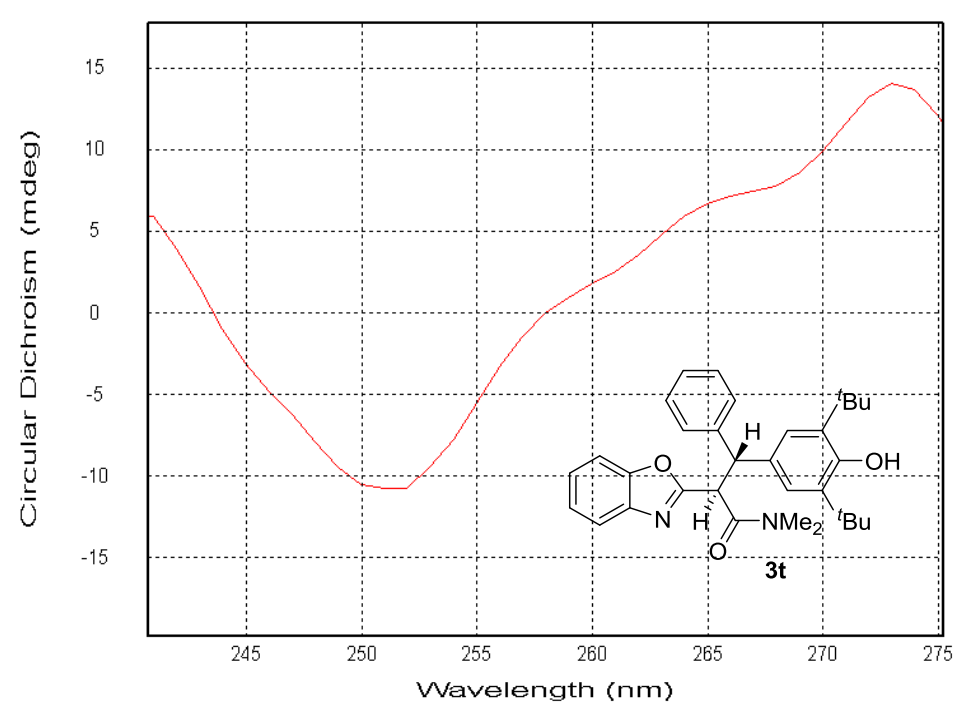

\section{Copies of NMR-Spectra of substrates}

$\underbrace{0.00}$<smiles>O=C1C=C(Cc2ccc(I)cc2)C=C(Br)C1Br</smiles>
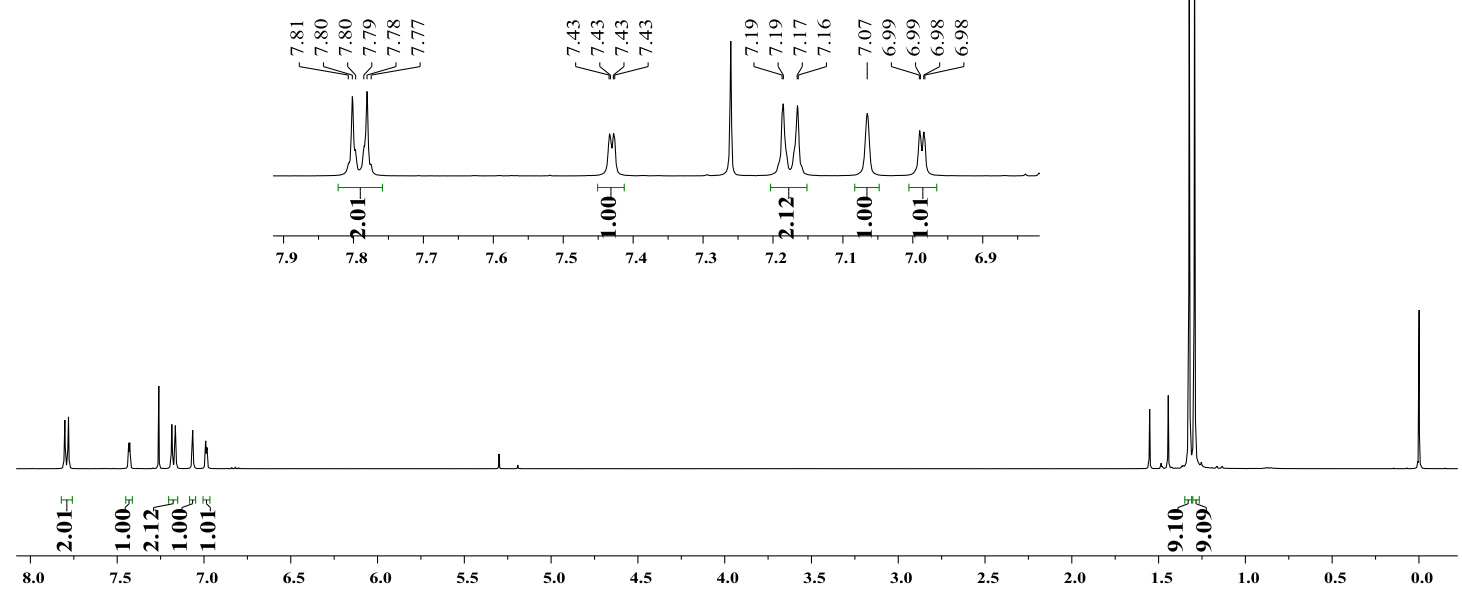

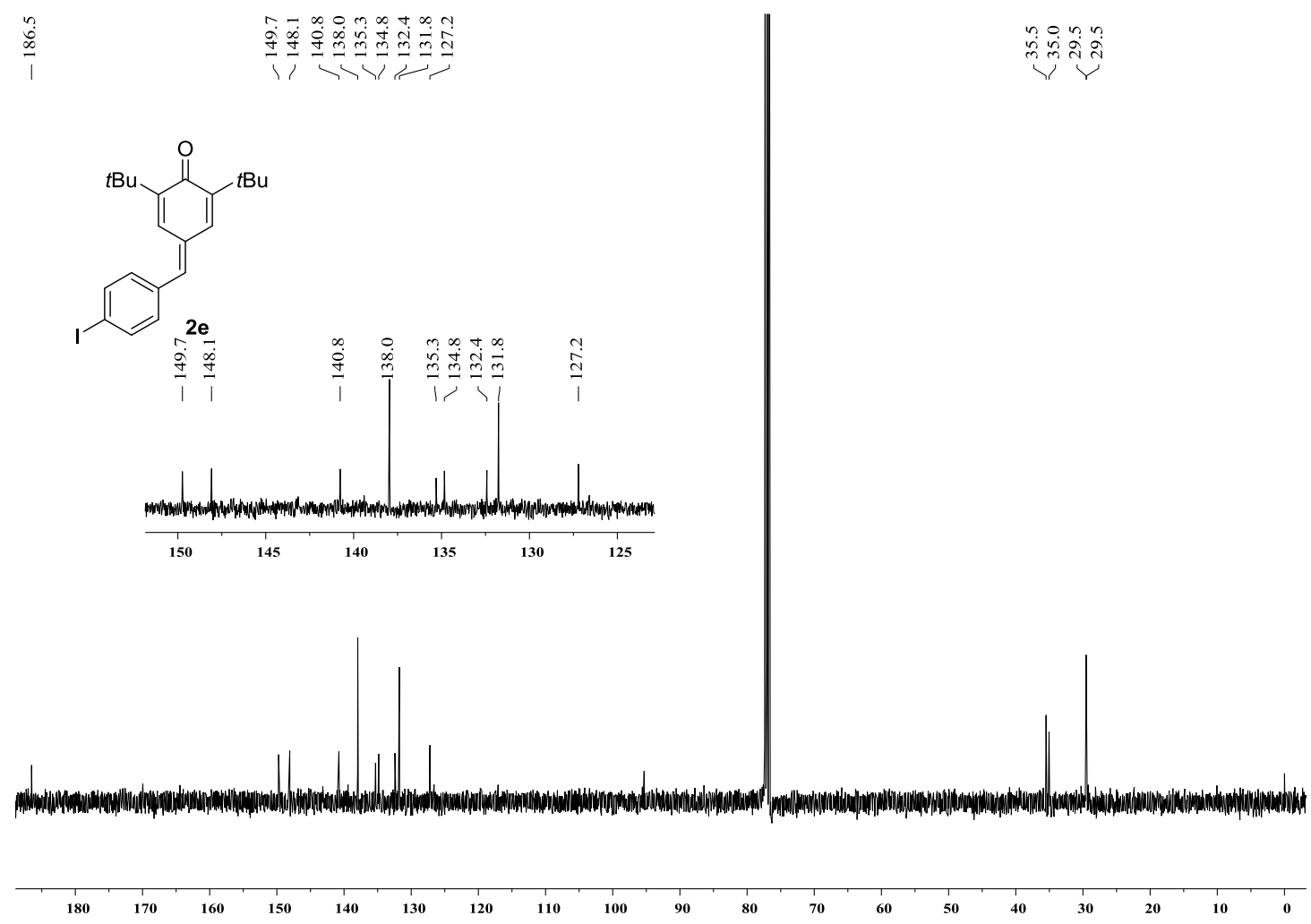

$\underbrace{2}$<smiles>CC(C)(C)C1=CC(=Cc2ccc3ccccc3n2)C=C(C(C)(C)C)C1=O</smiles>

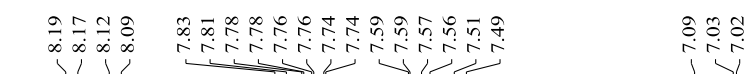
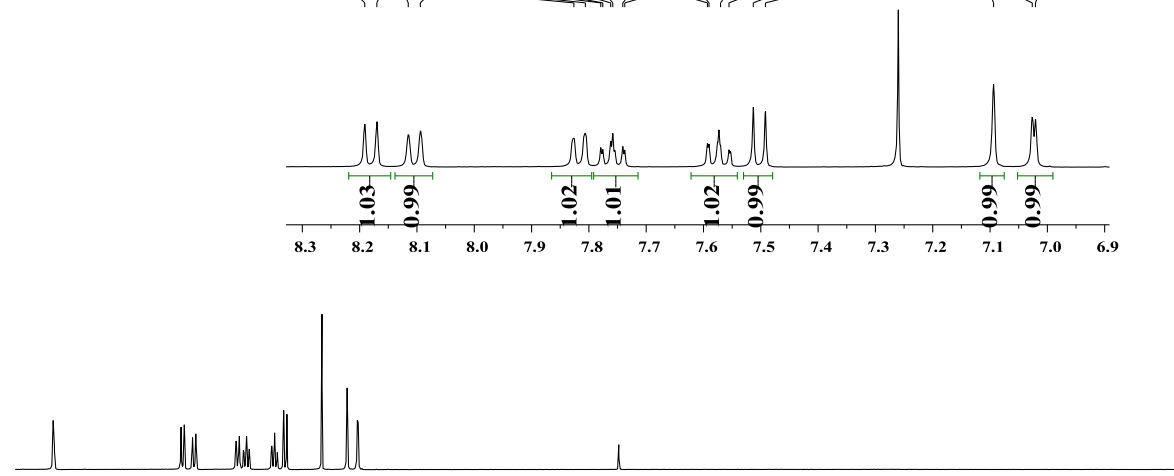

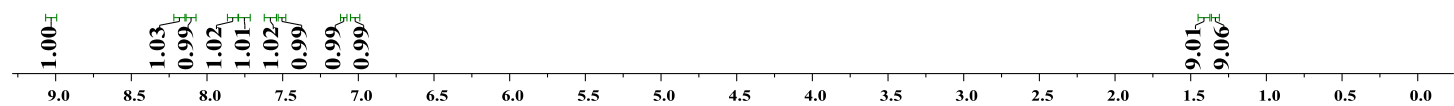


<smiles>CC(C)(C)C1=CC(=Cc2ccc3ccccc3n2)C=C(C(C)(C)C)C1=O</smiles>

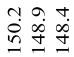

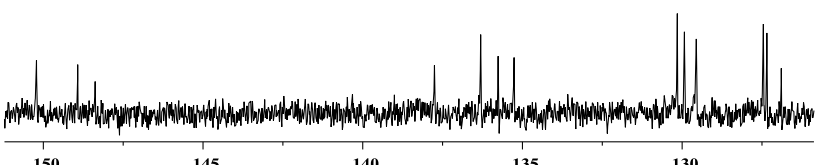
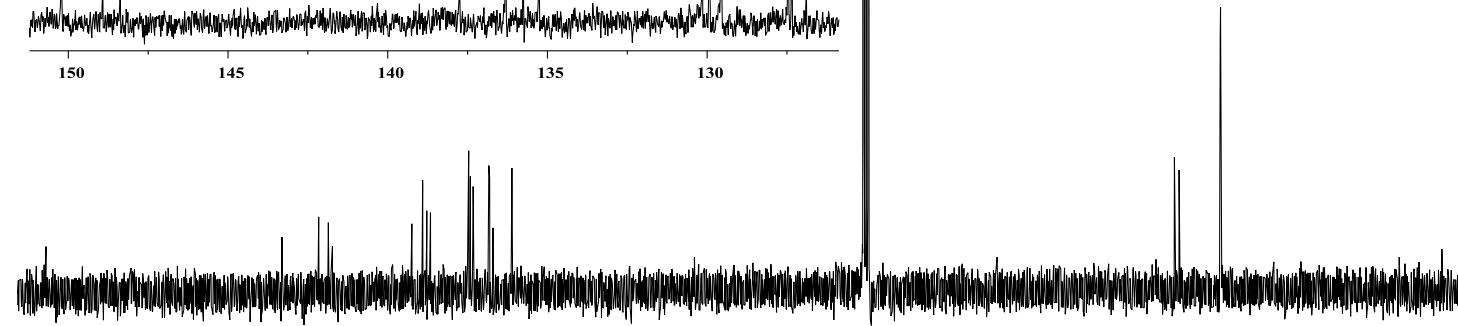

$\begin{array}{llllllllllllllllllll}90 & 180 & 170 & 160 & 150 & 140 & 130 & 120 & 110 & 100 & 90 & 80 & 70 & 60 & 50 & 40 & 30 & 20 & 10 & 0\end{array}$

\section{Copies of NMR-Spectra of products}

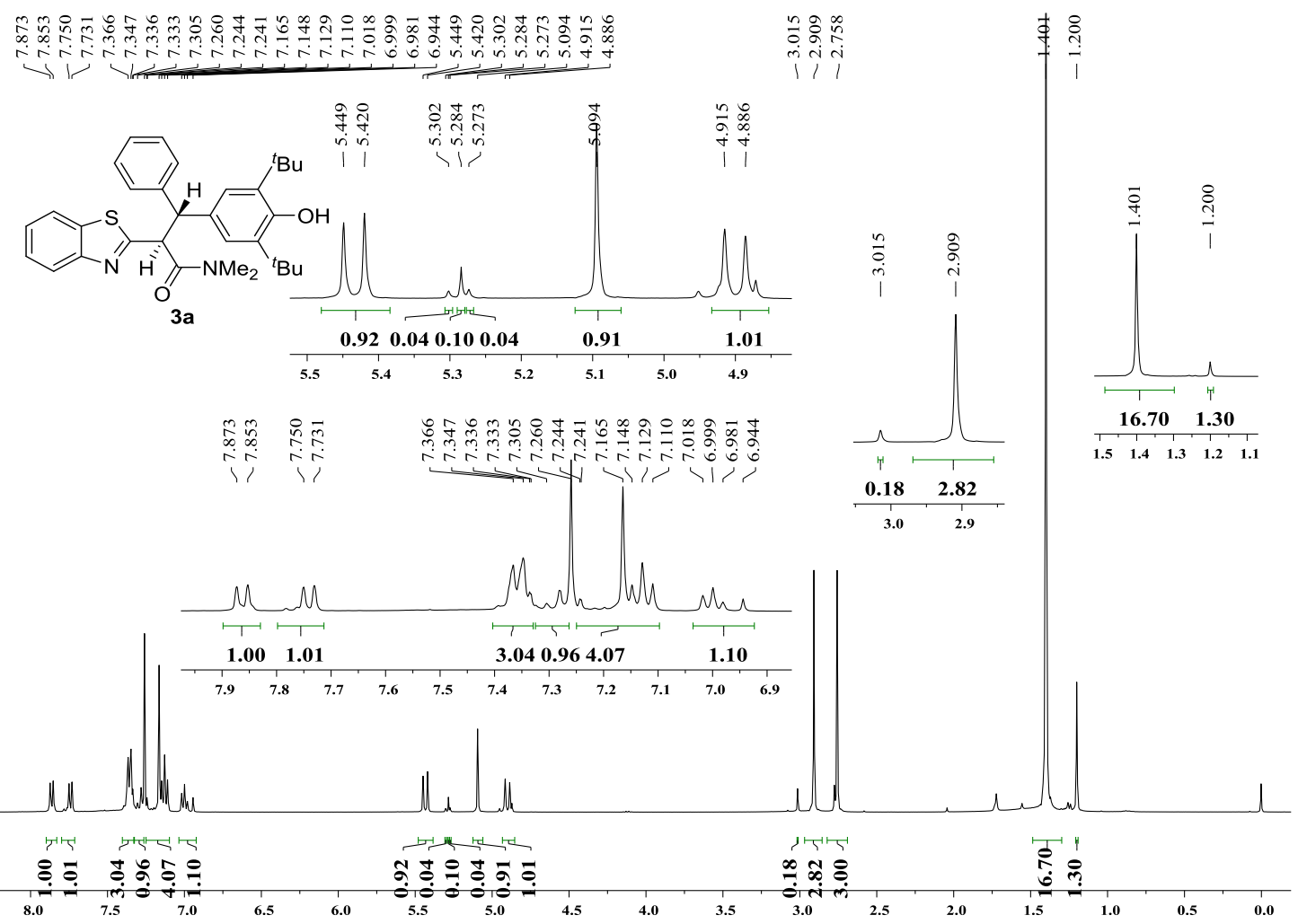




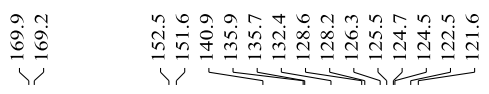

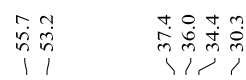<smiles>CCC(C)c1cc(C(C)(C)C)cc(C(C(=O)O)C(c2ccccc2)[C@@H](c2ccccc2)c2nc3ccccc3s2)c1O</smiles>

$3 a$
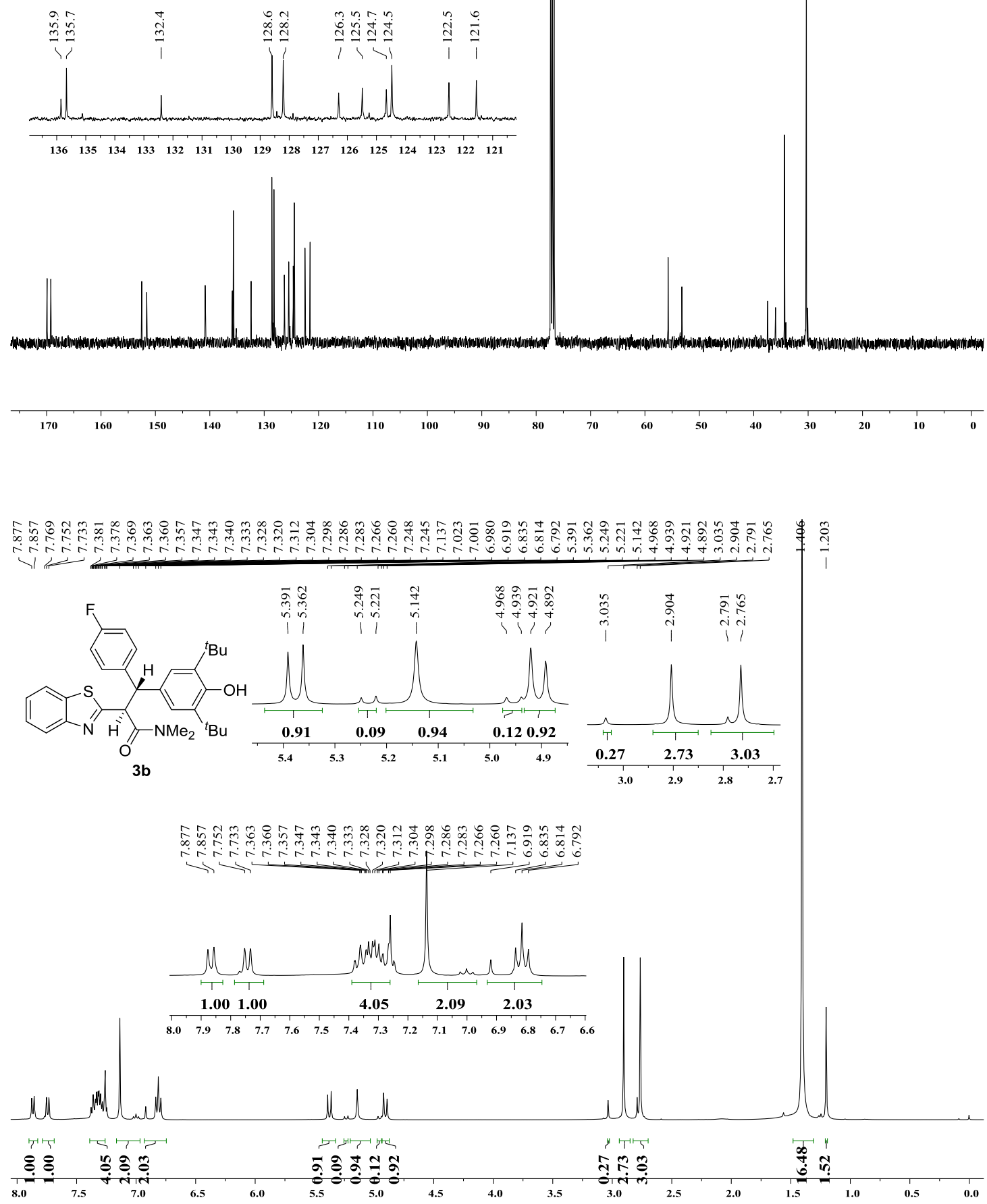


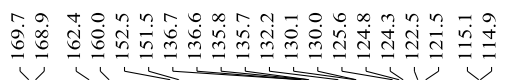

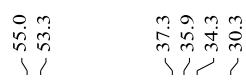

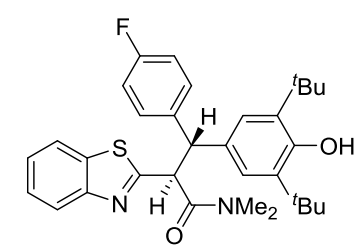

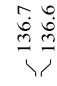

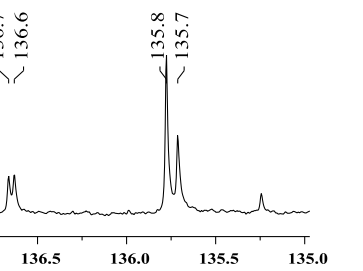

ำ
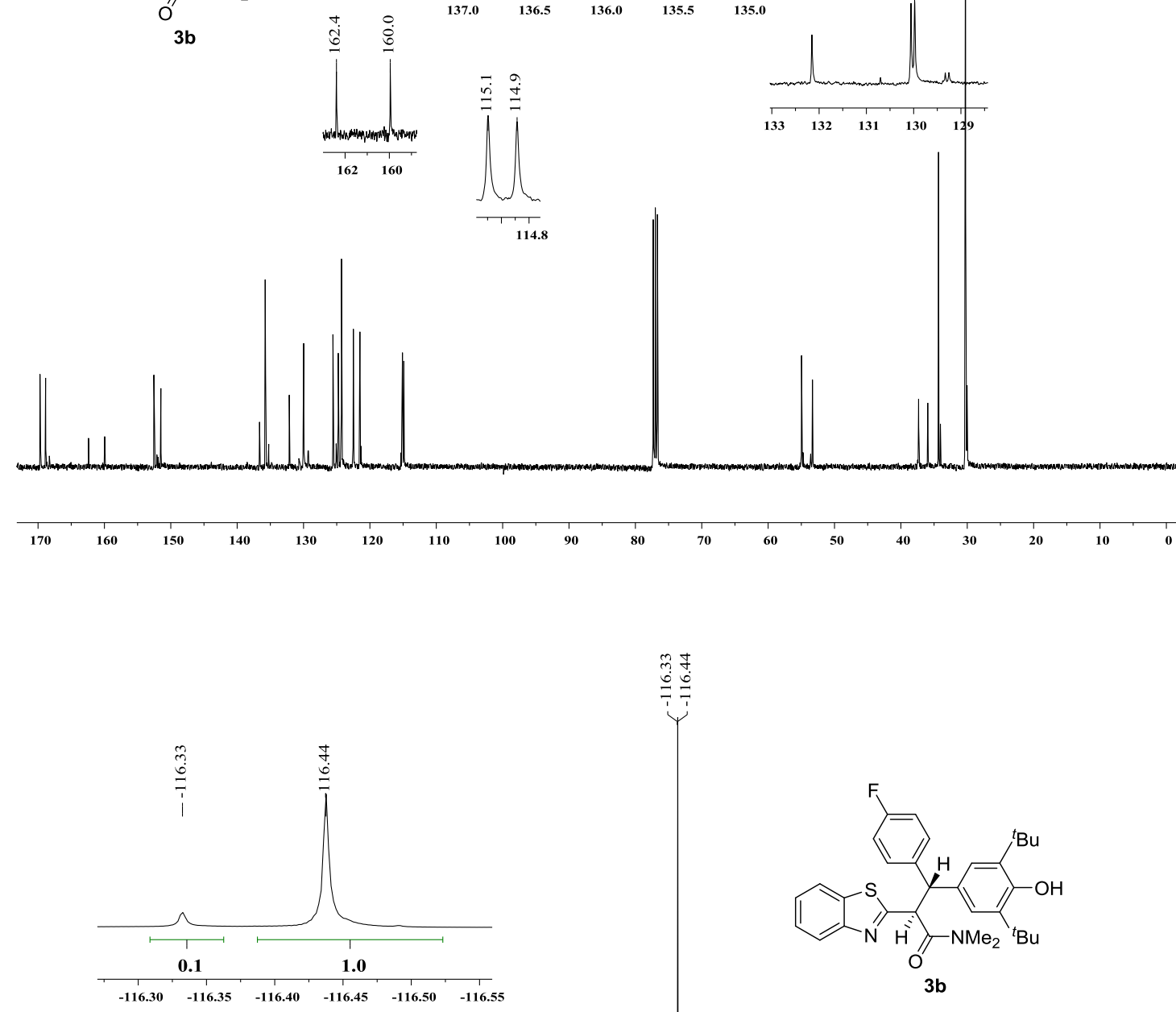

을

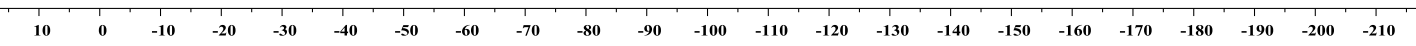



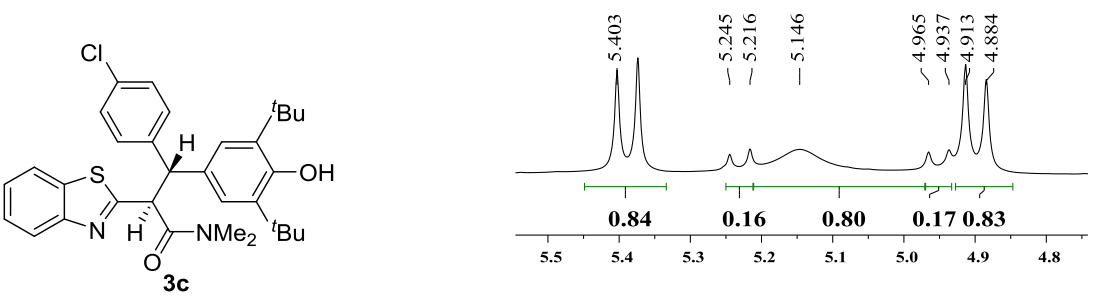

$\sqrt[5]{15}$

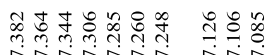

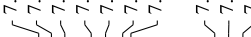

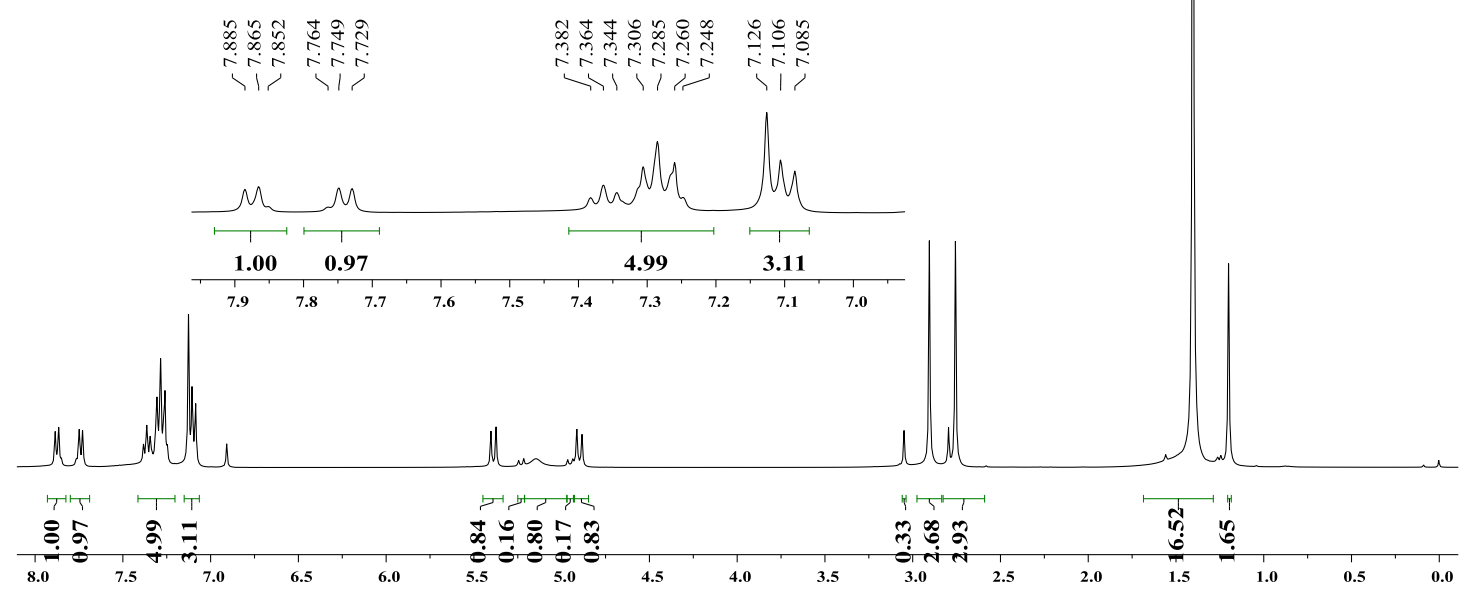

ond

争

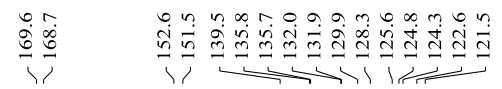
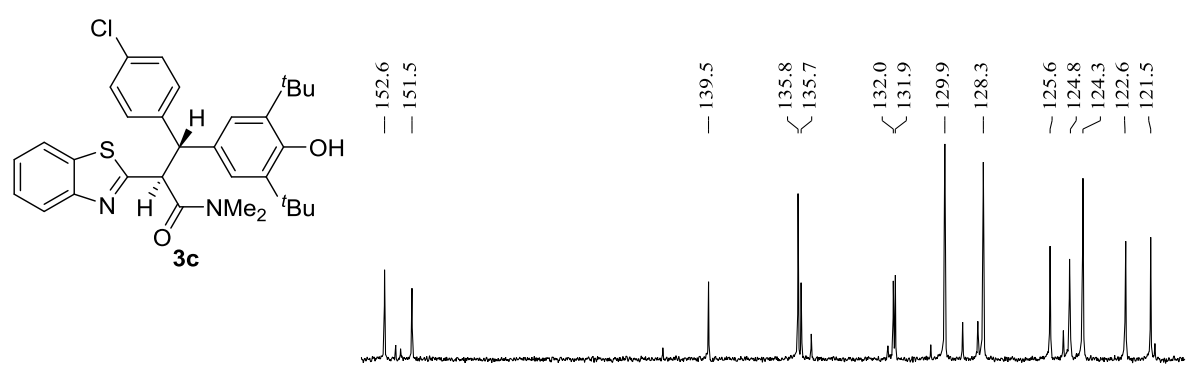

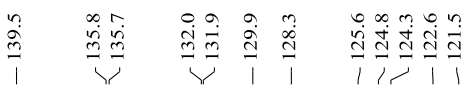

$\begin{array}{llllllllllllllll}152 & 150 & 148 & 146 & 144 & 142 & 140 & 138 & 136 & 134 & 132 & 130 & 128 & 126 & 124 & 122\end{array}$

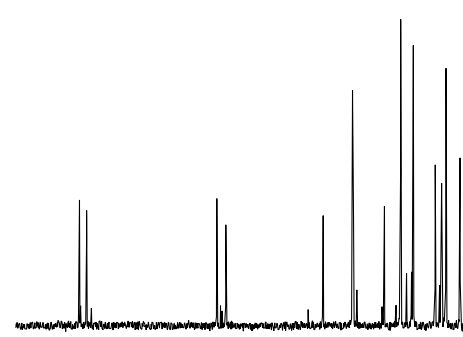

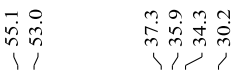

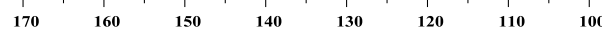

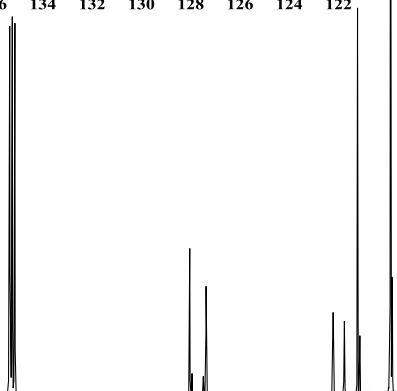



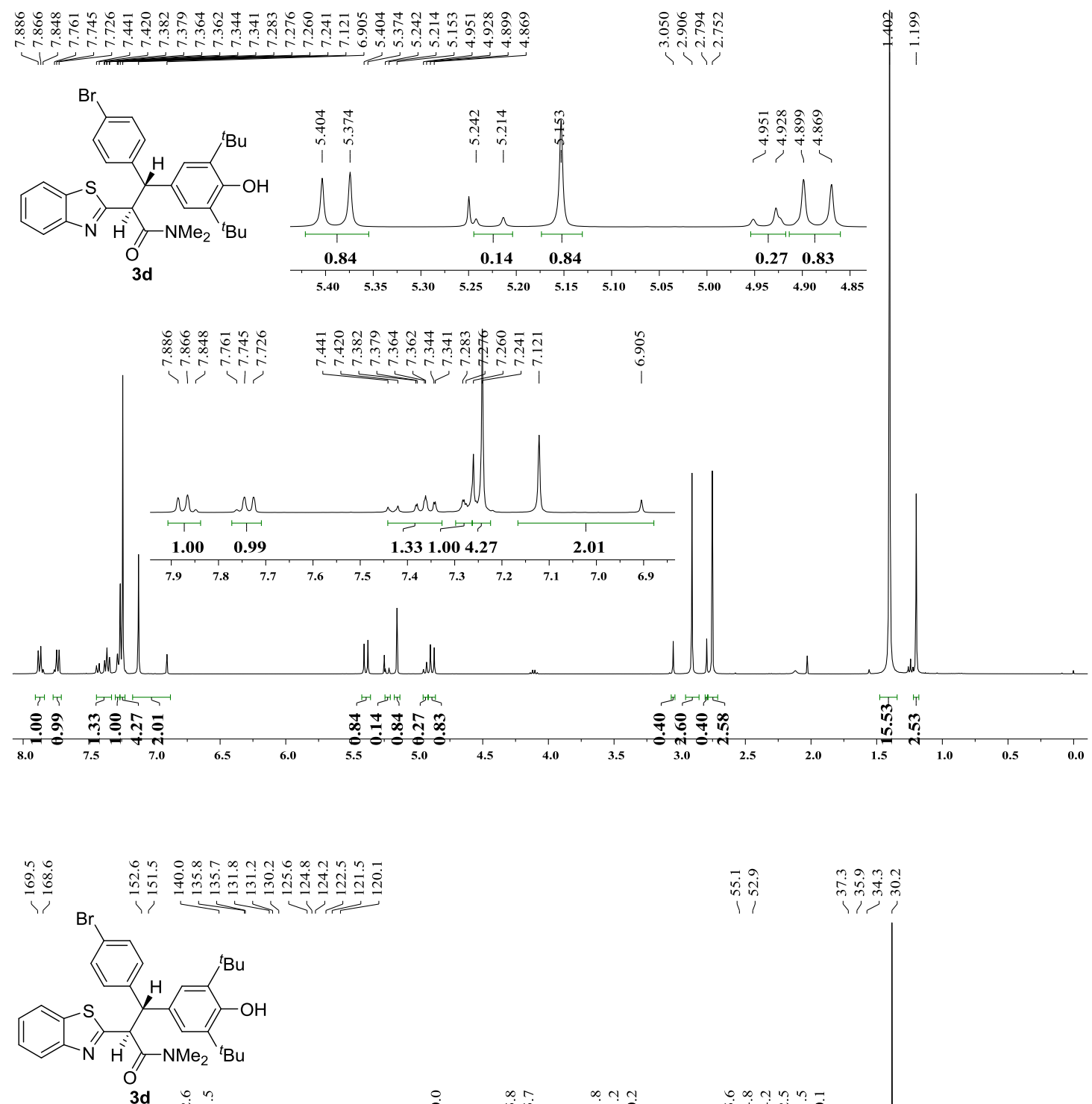

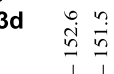

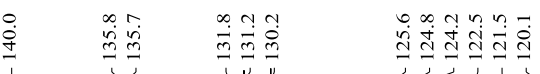
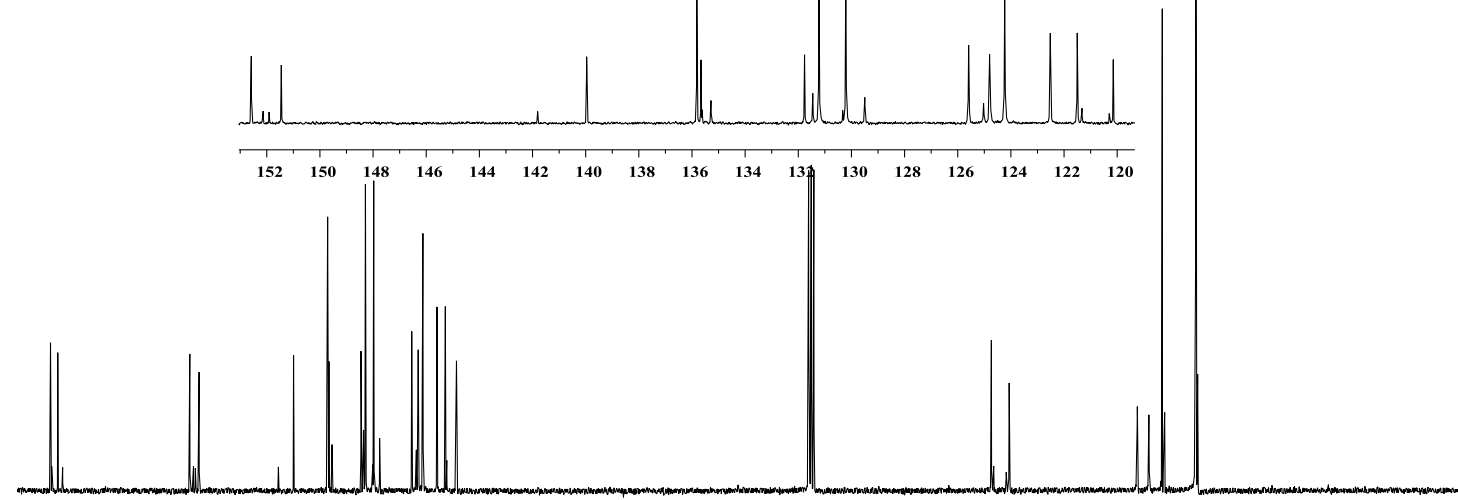

17

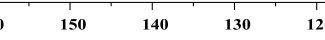




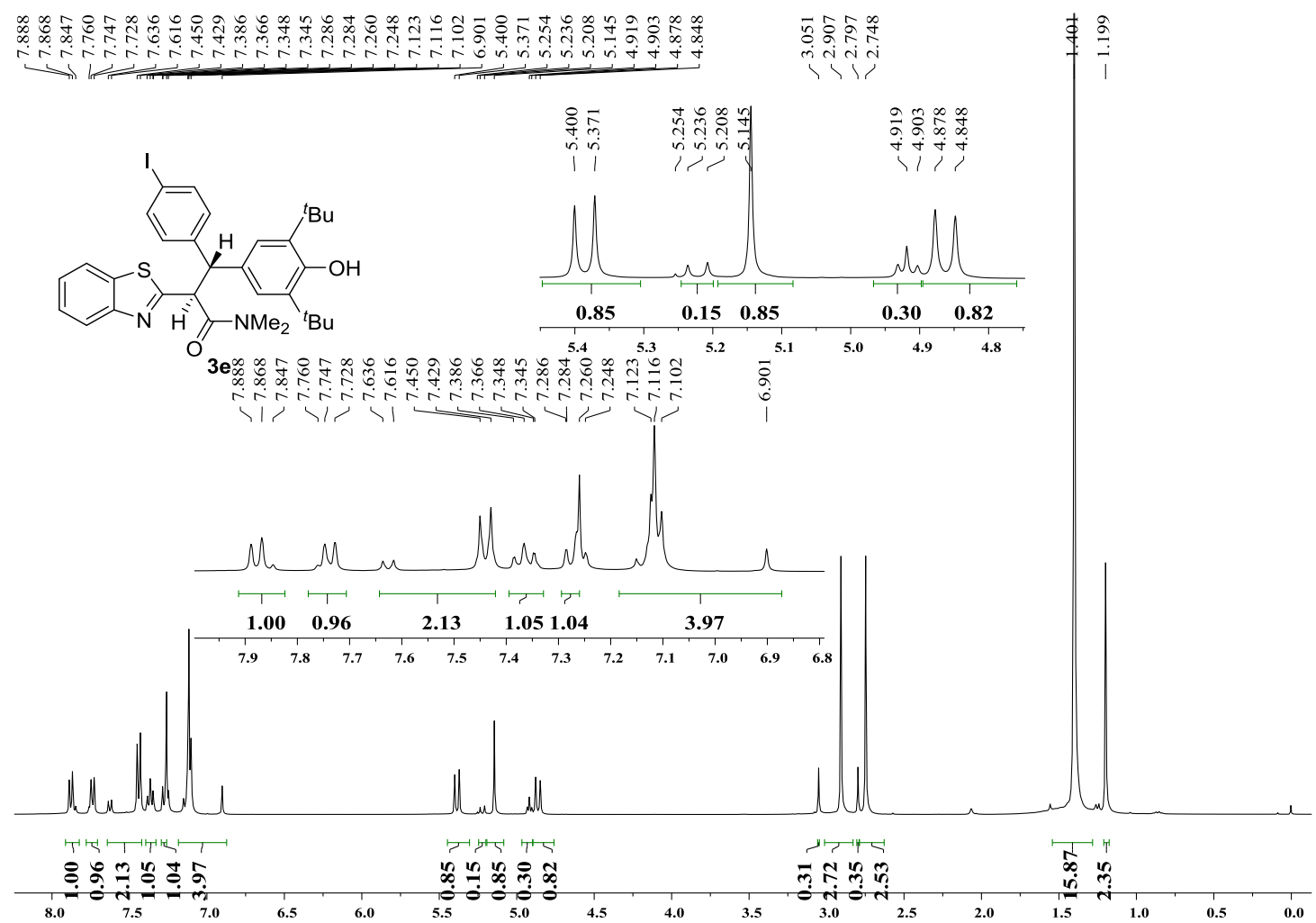

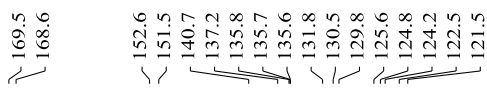
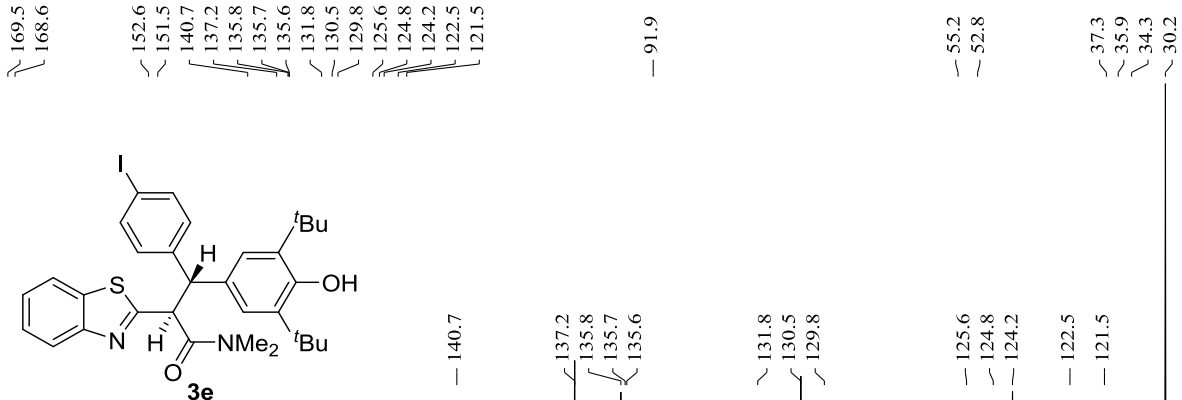

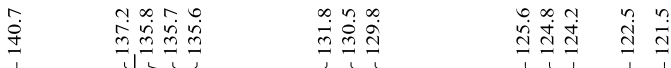
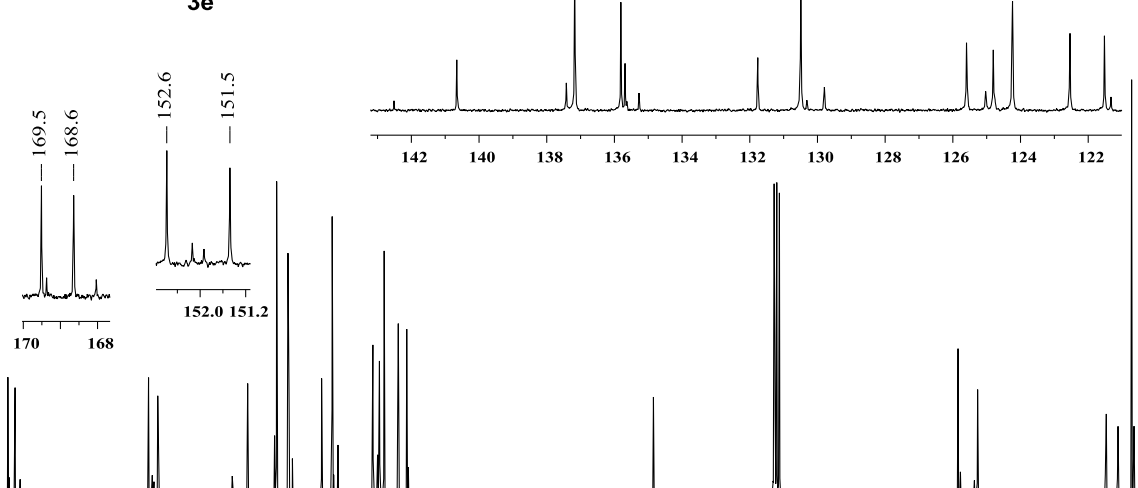

170

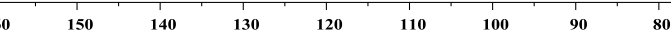



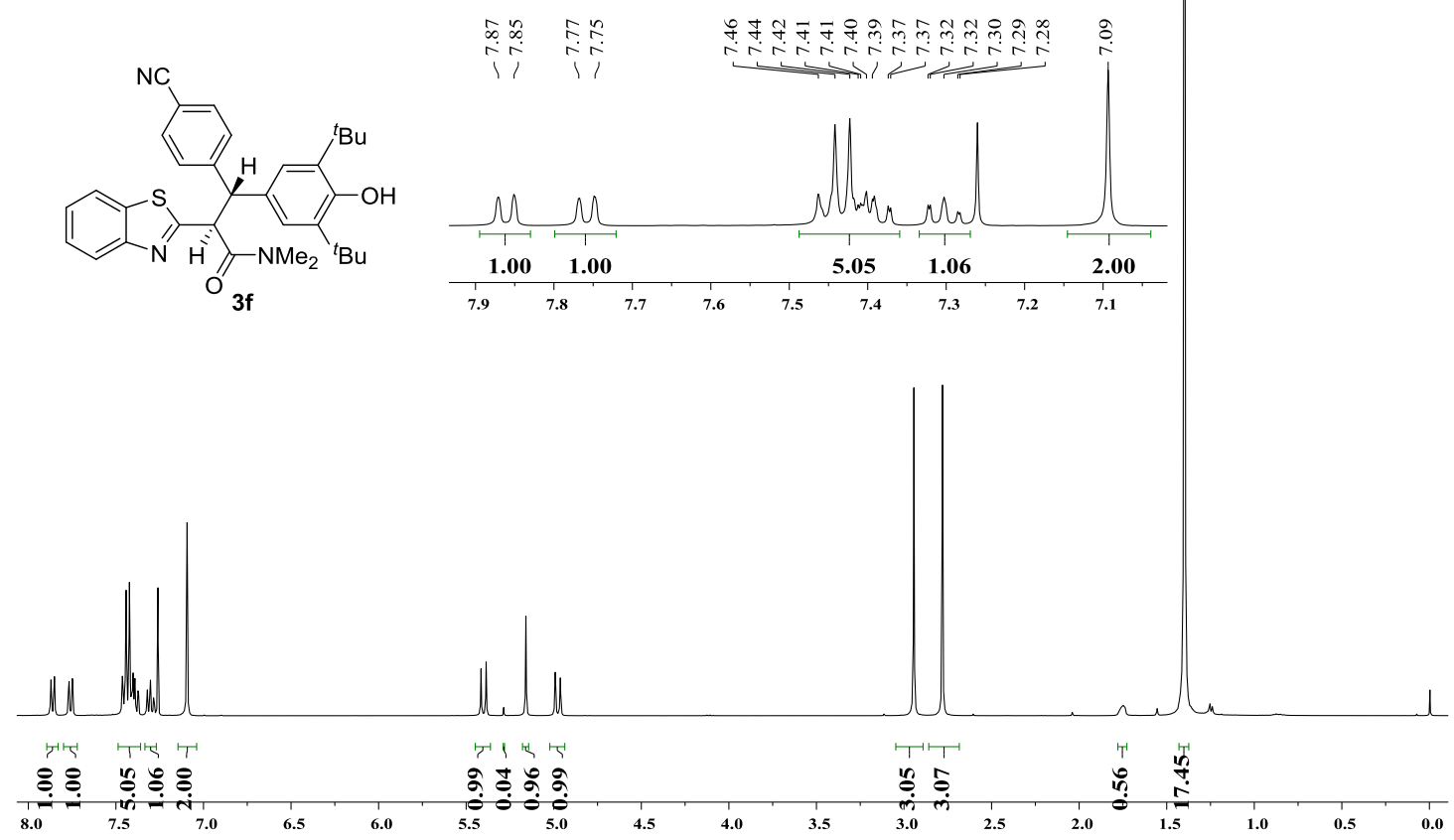

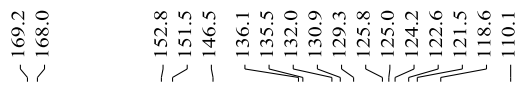
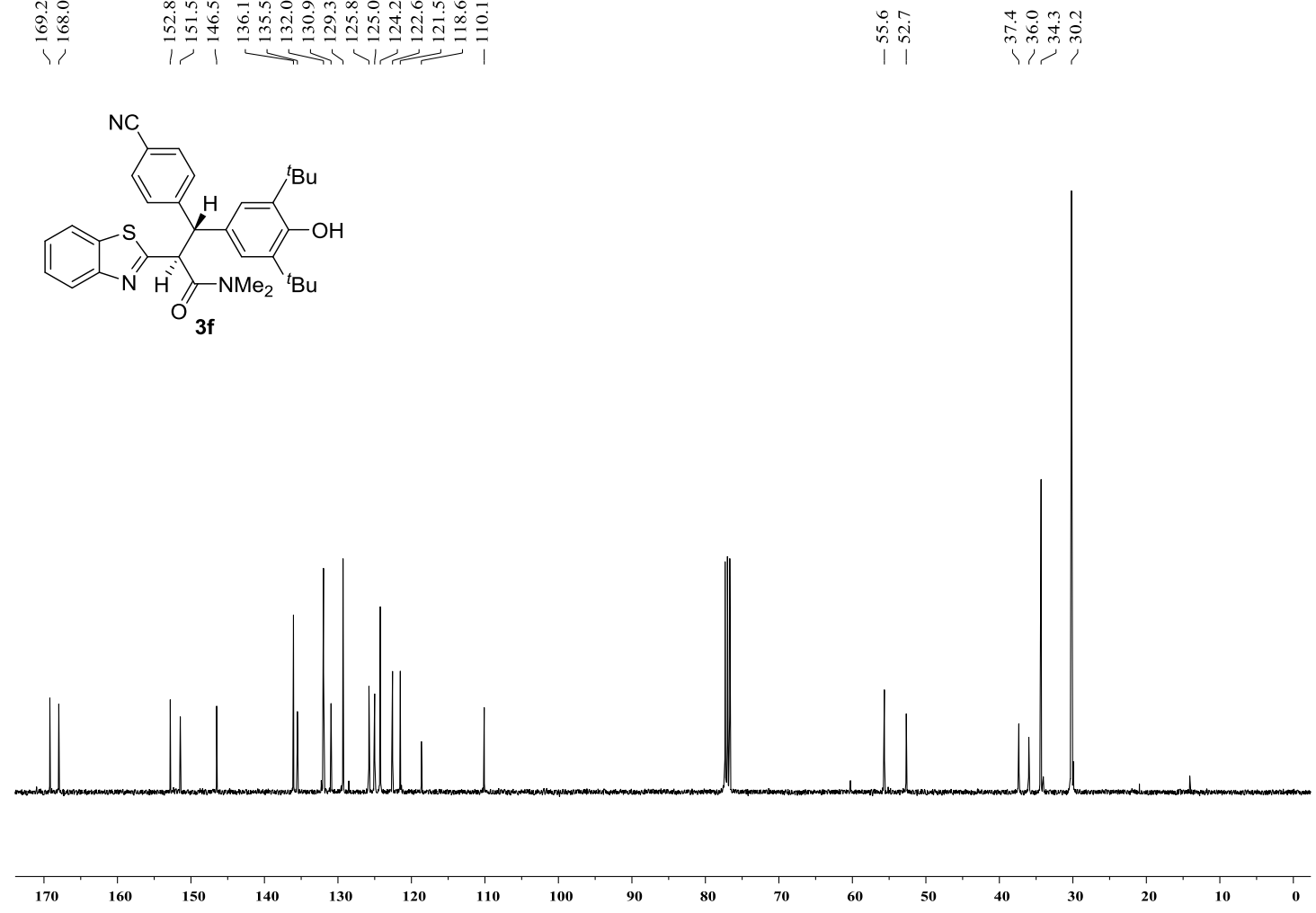

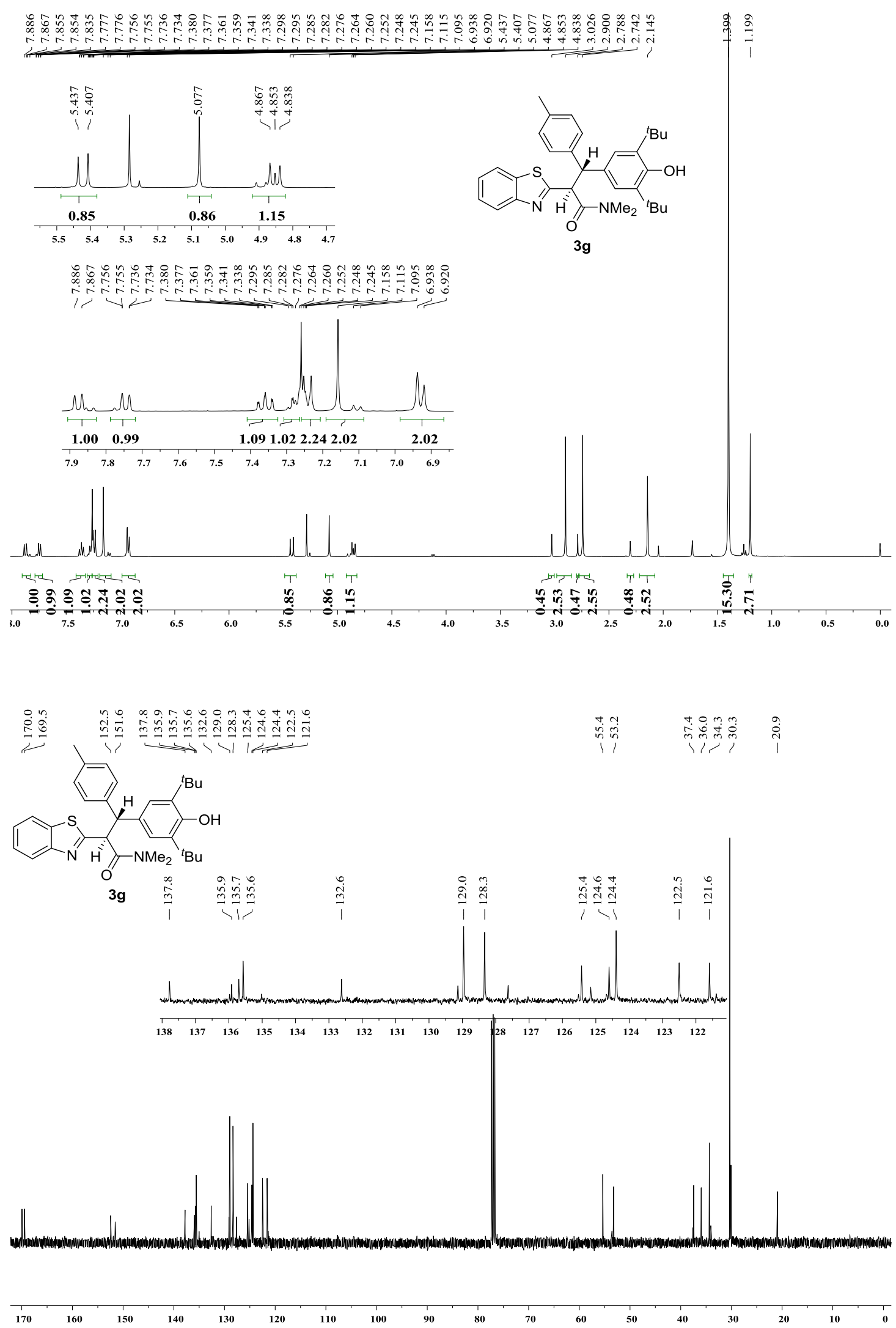


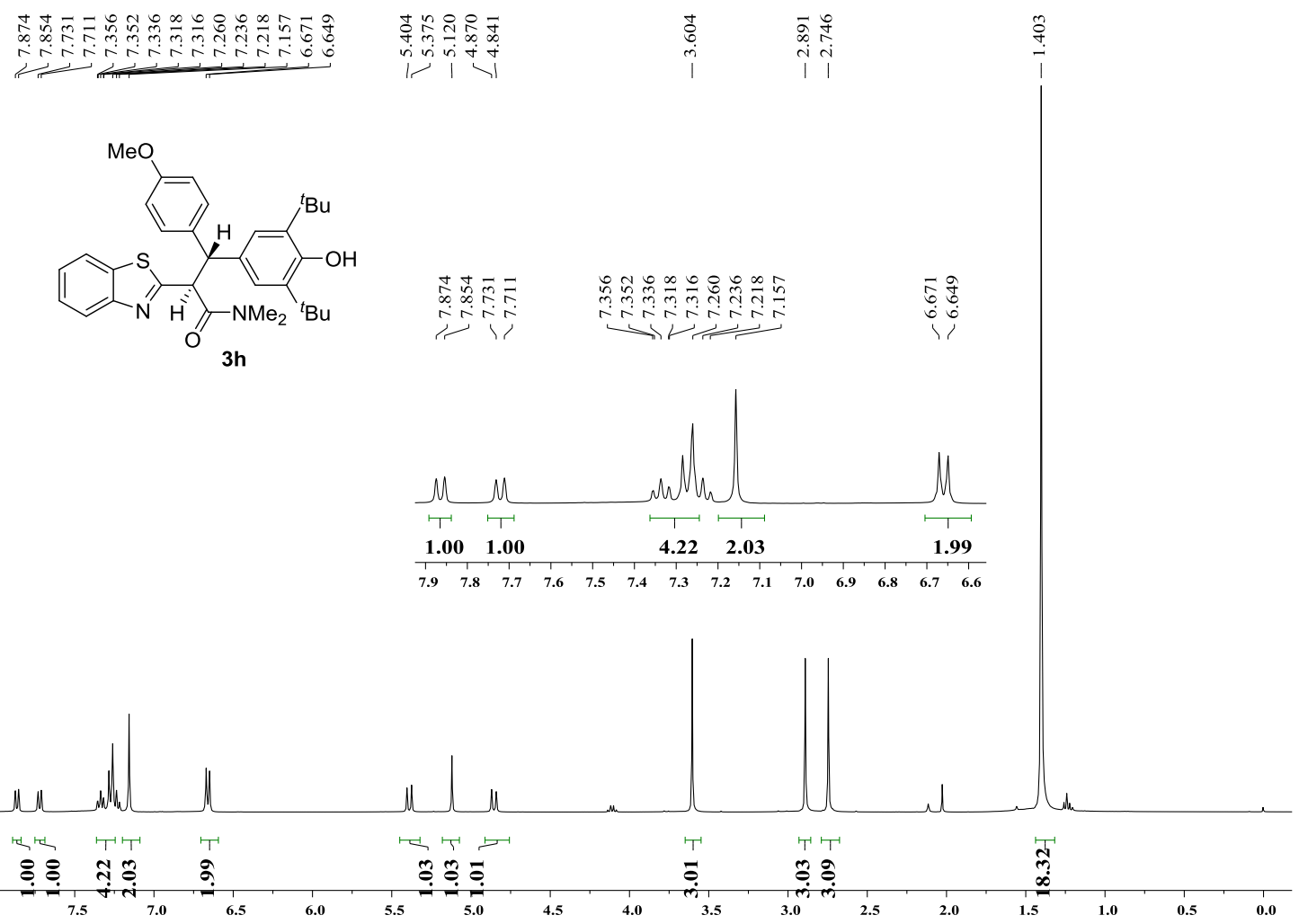

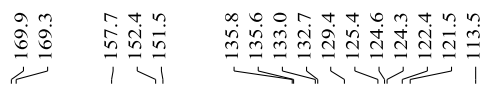
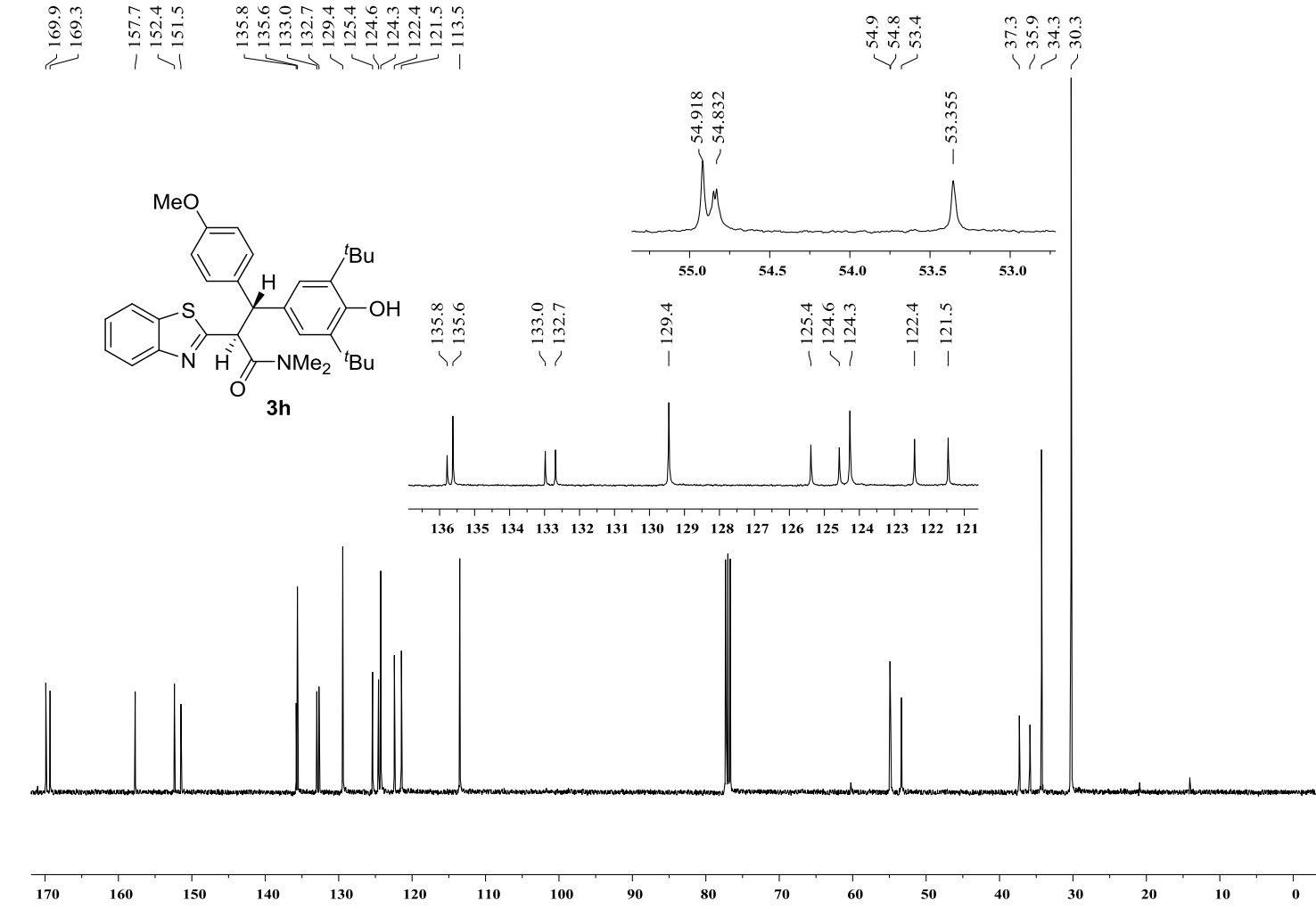


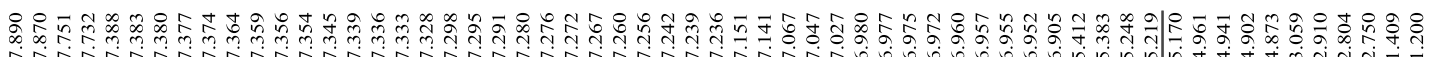

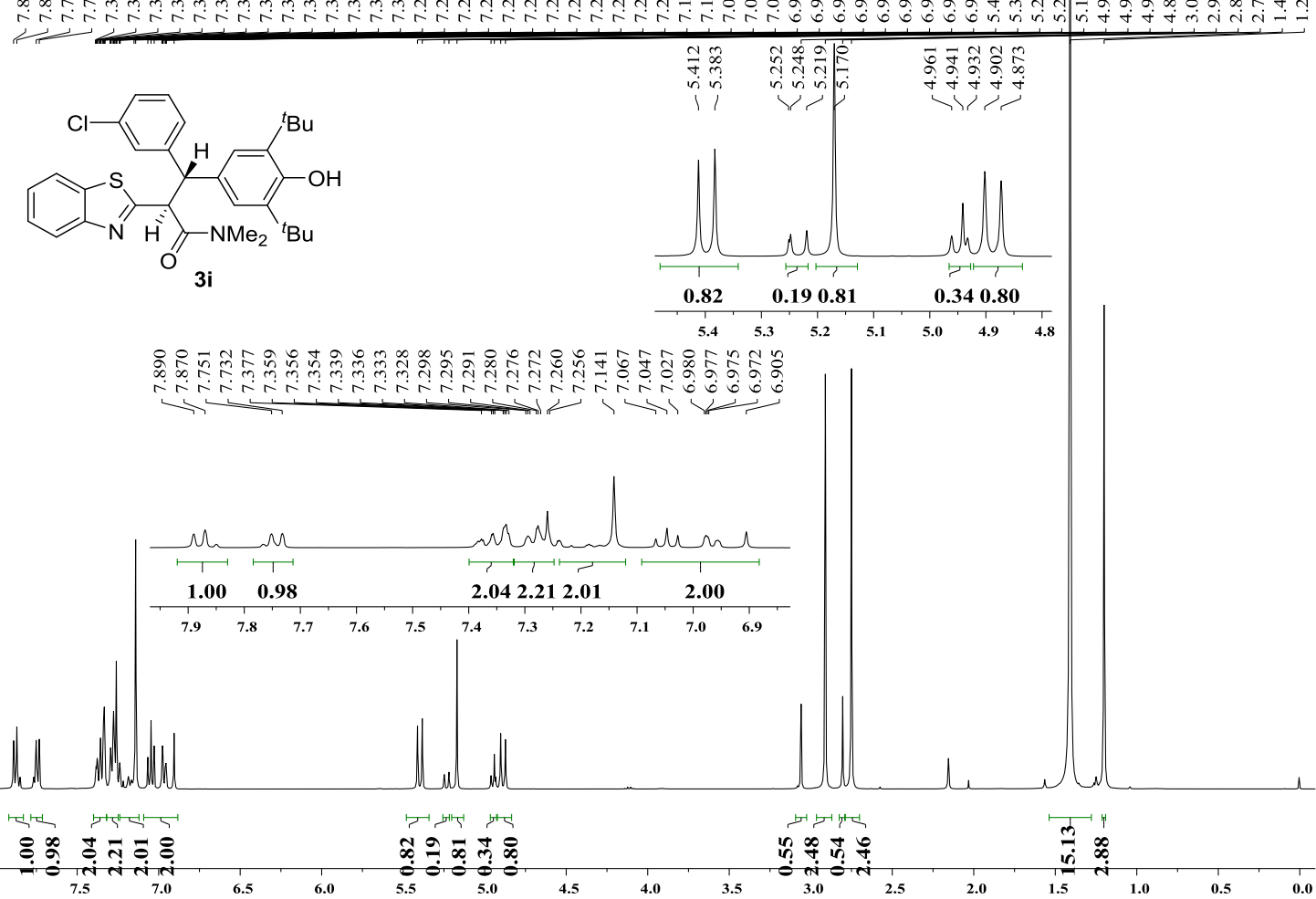

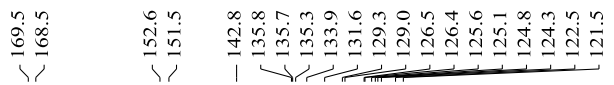

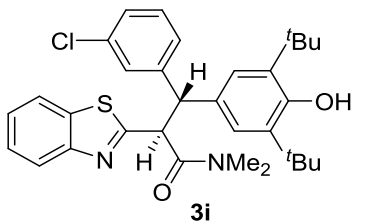

$\underset{\substack{0\\}}{\substack{n \\ 1}}$

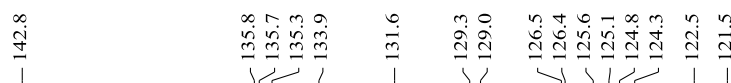

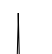
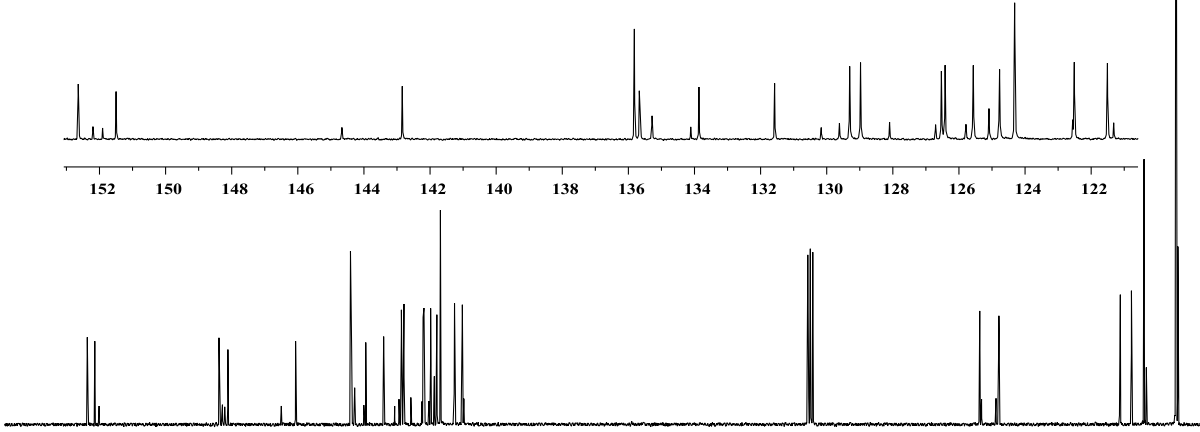

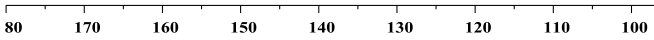




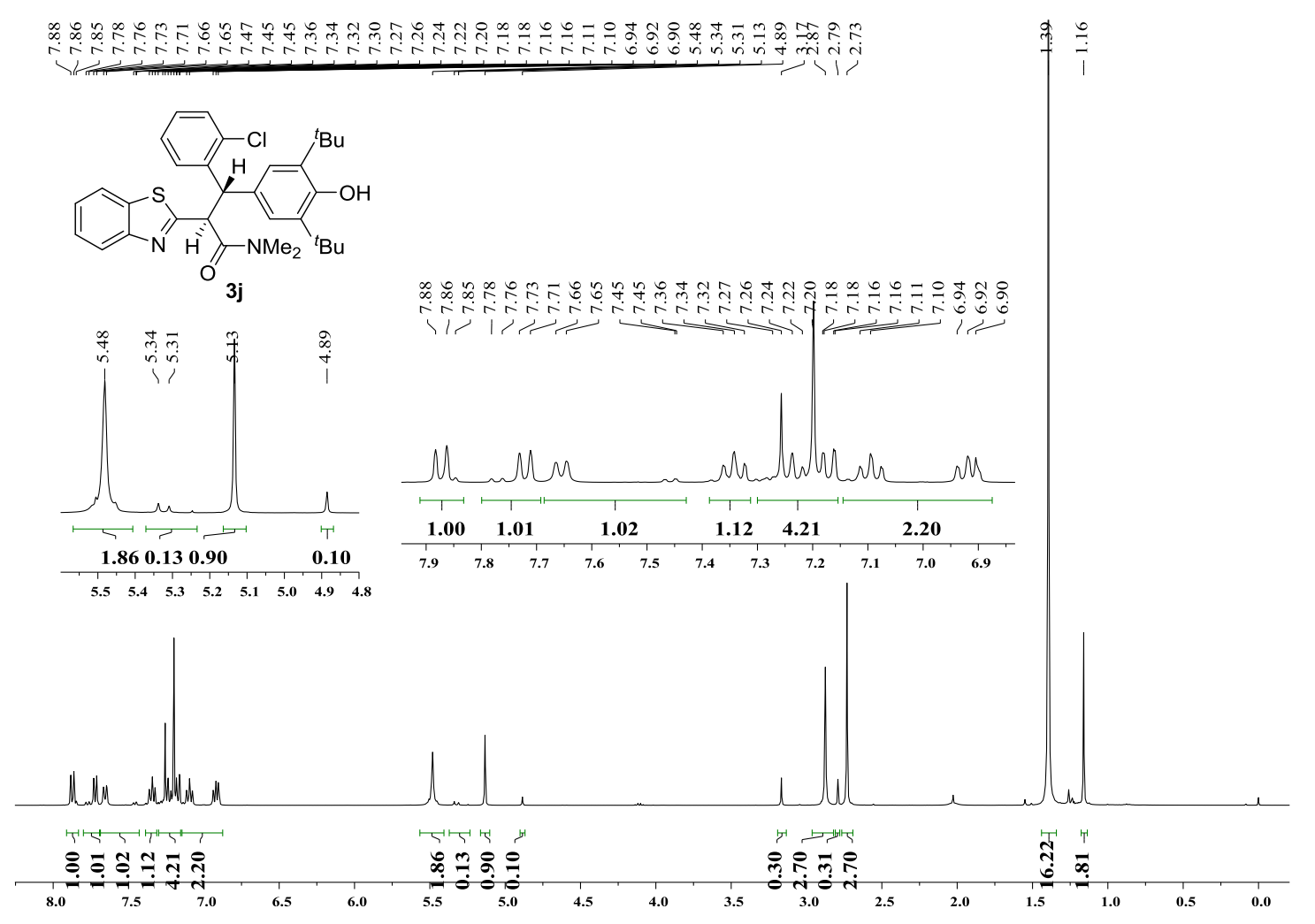

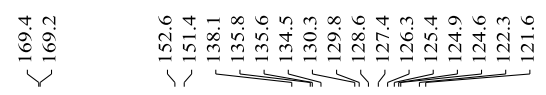<smiles>CNC(=O)[C@@H](c1nc2ccccc2s1)[C@H](c1cc(C(C)(C)C)c(O)c(C(C)(C)C)c1)c1ccccc1Cl</smiles>

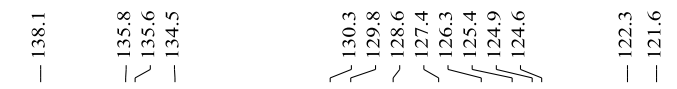

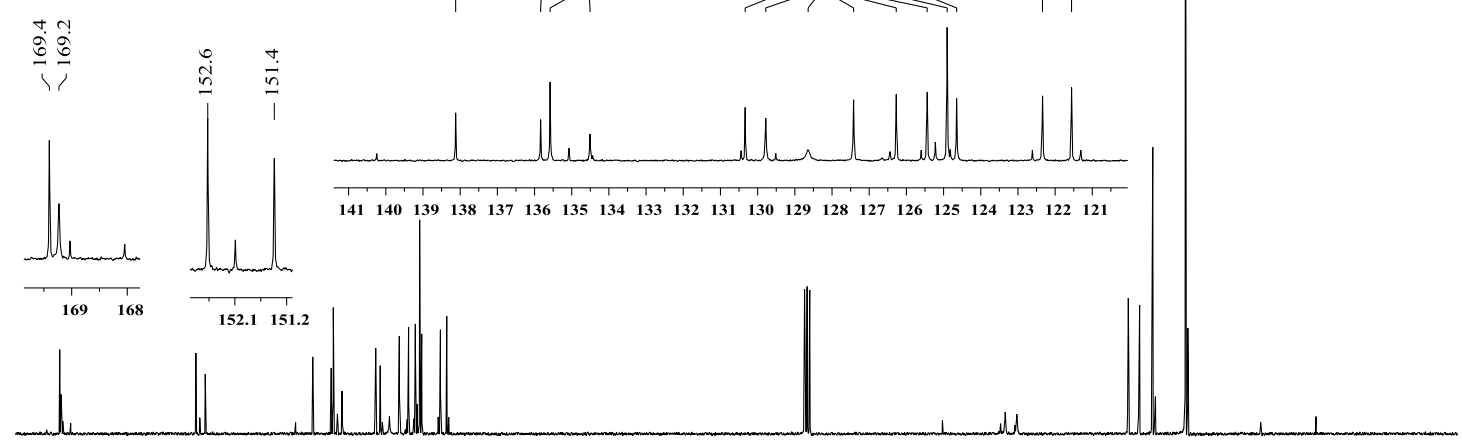

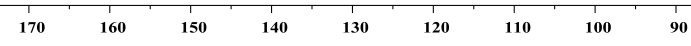




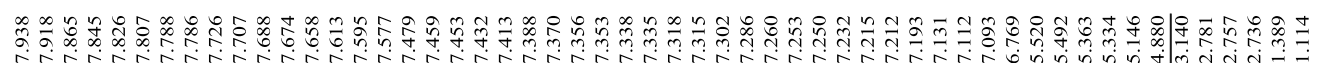

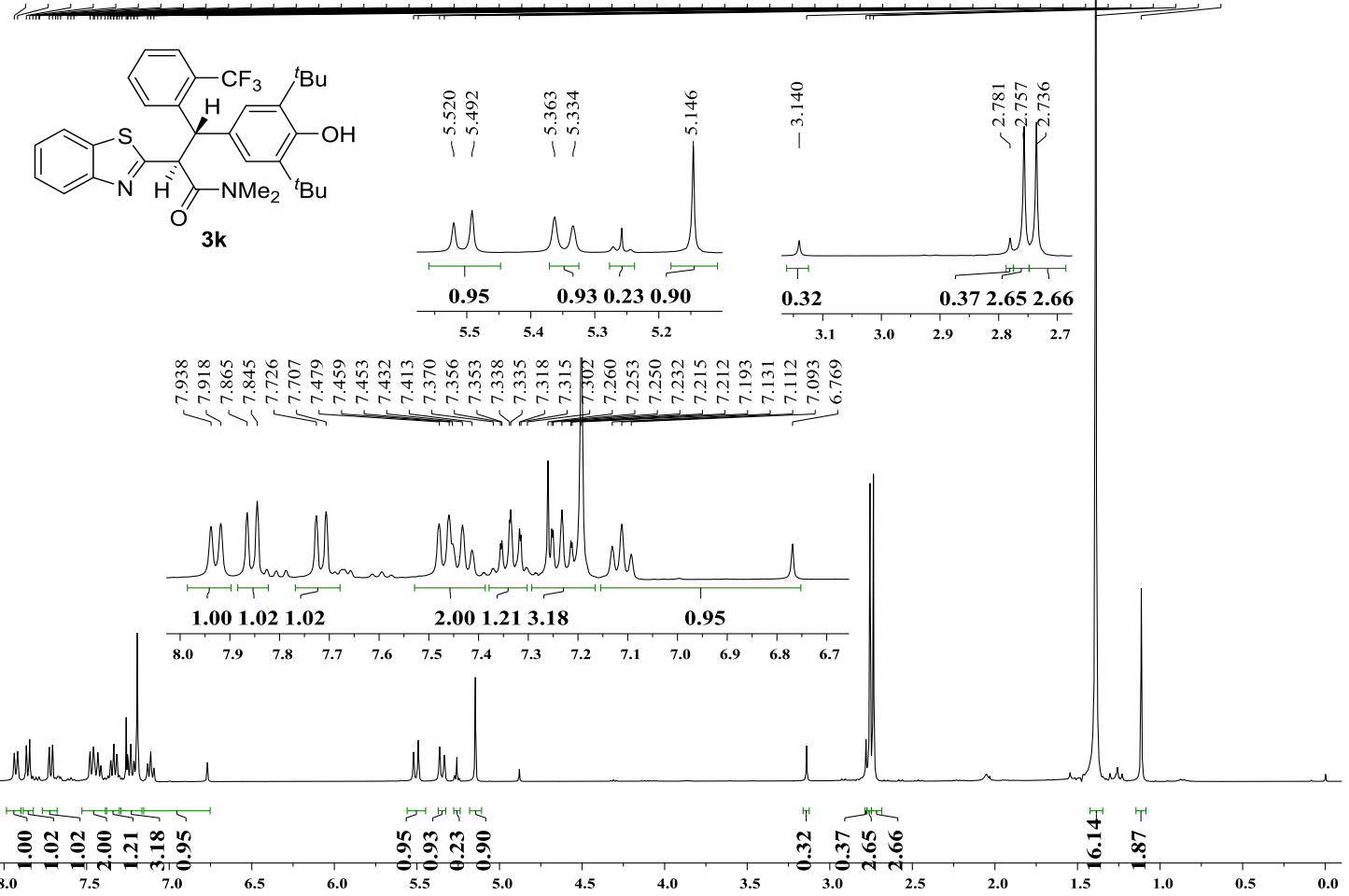

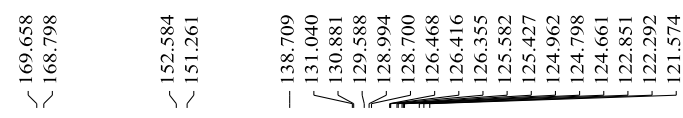
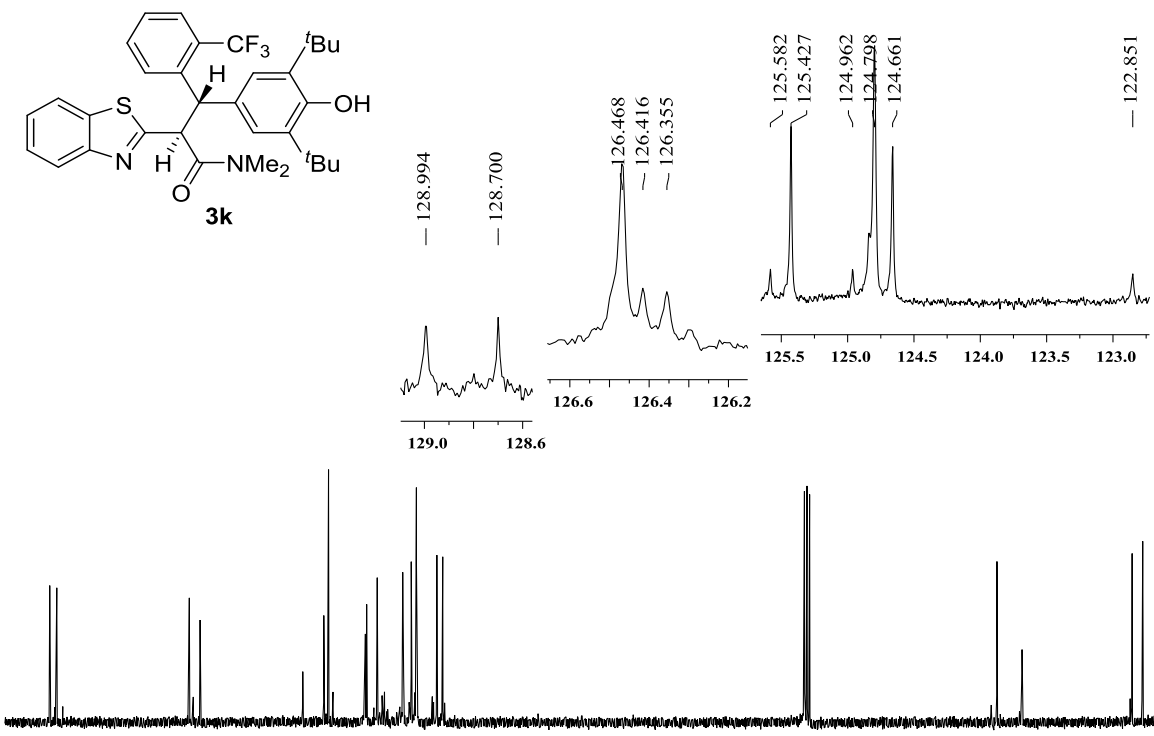

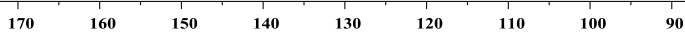




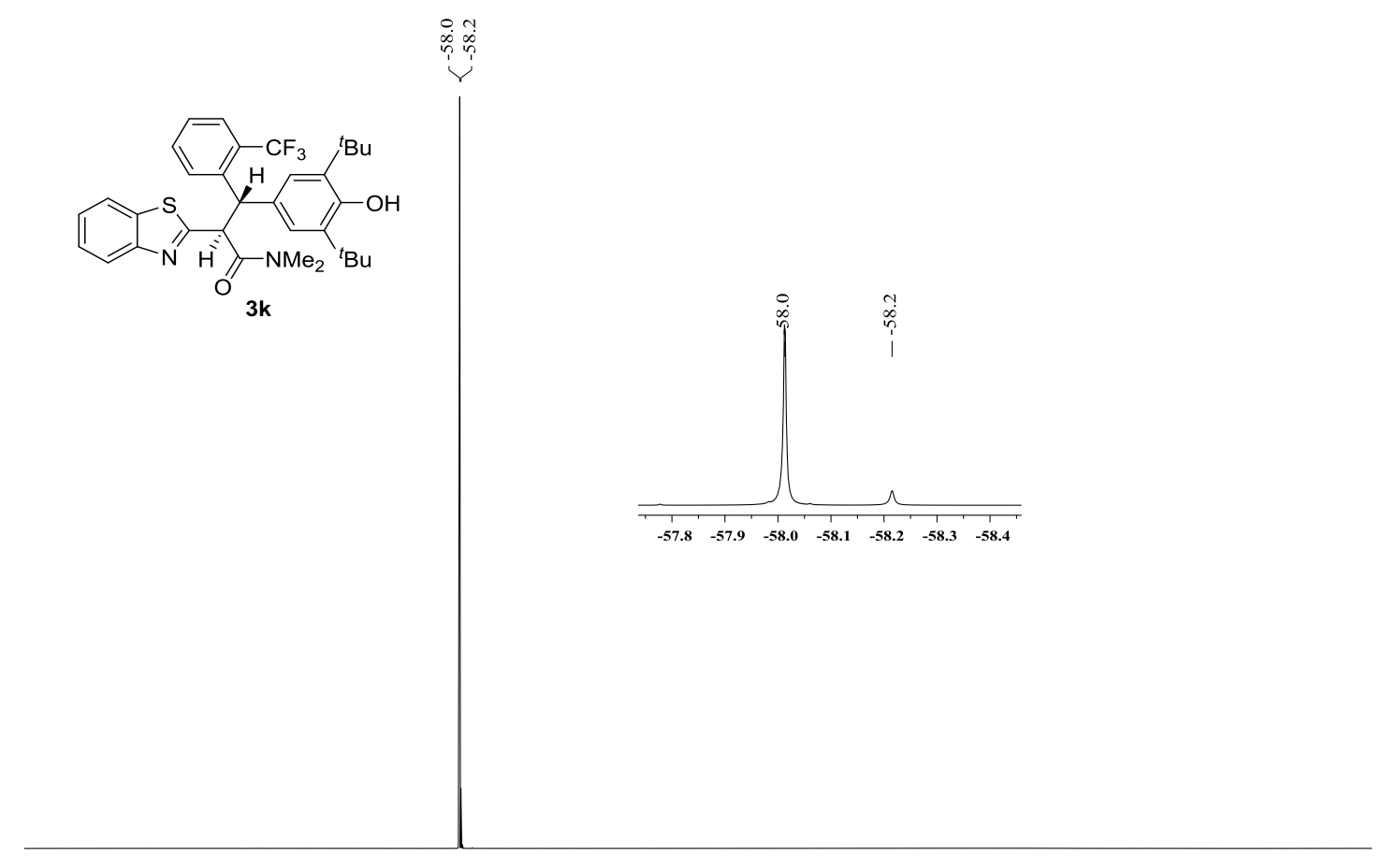

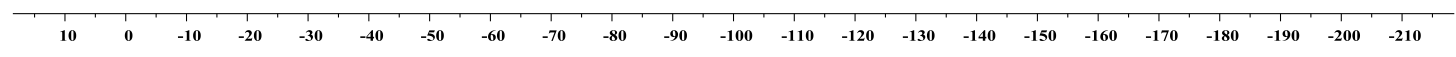

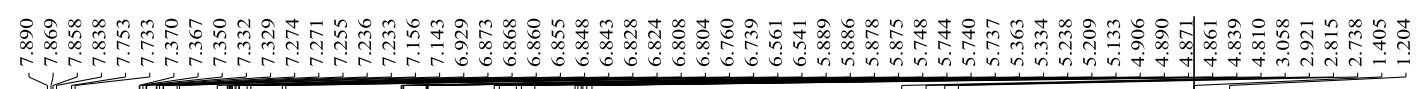
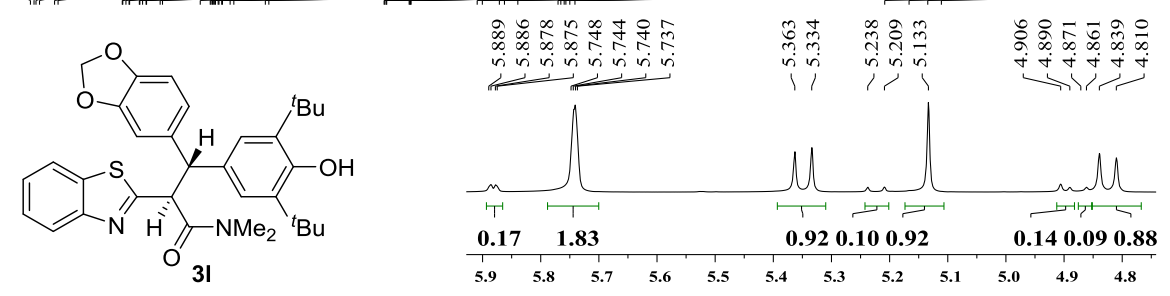

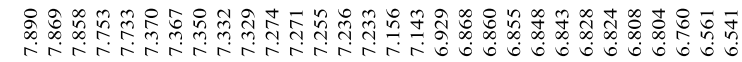
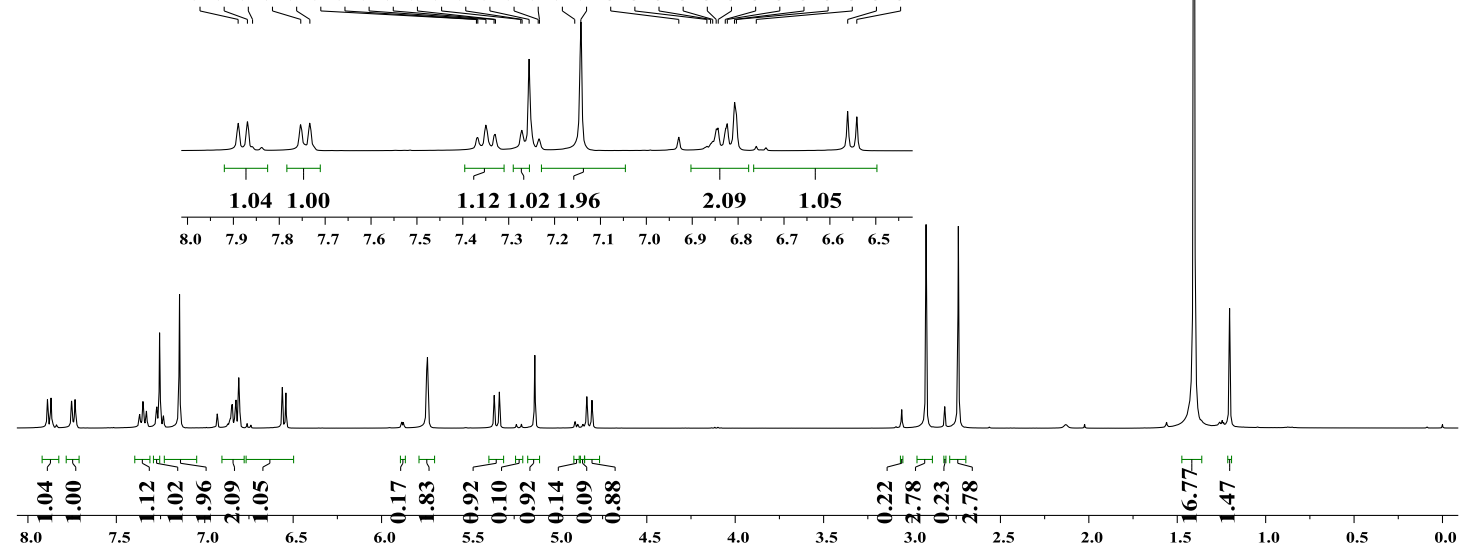

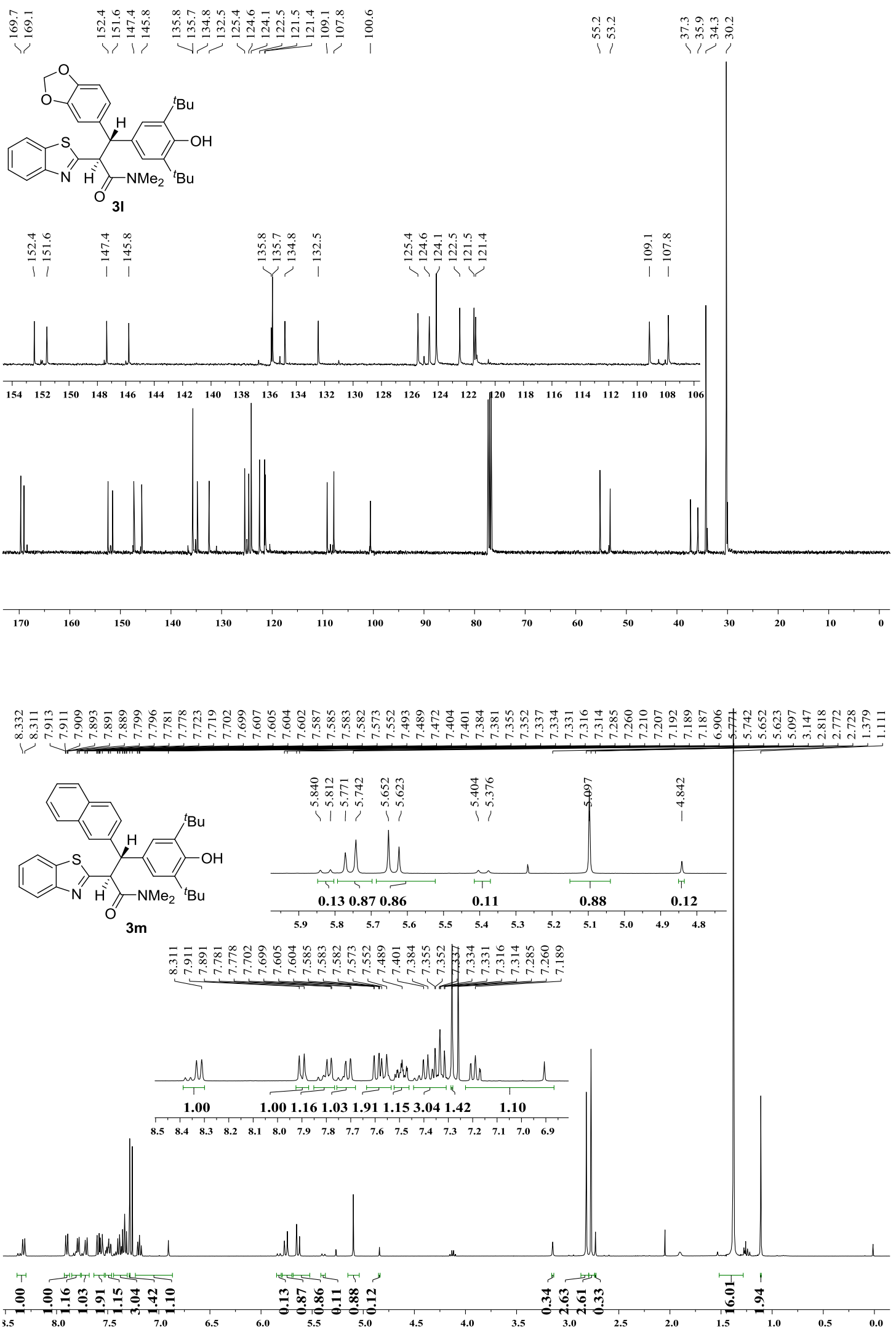

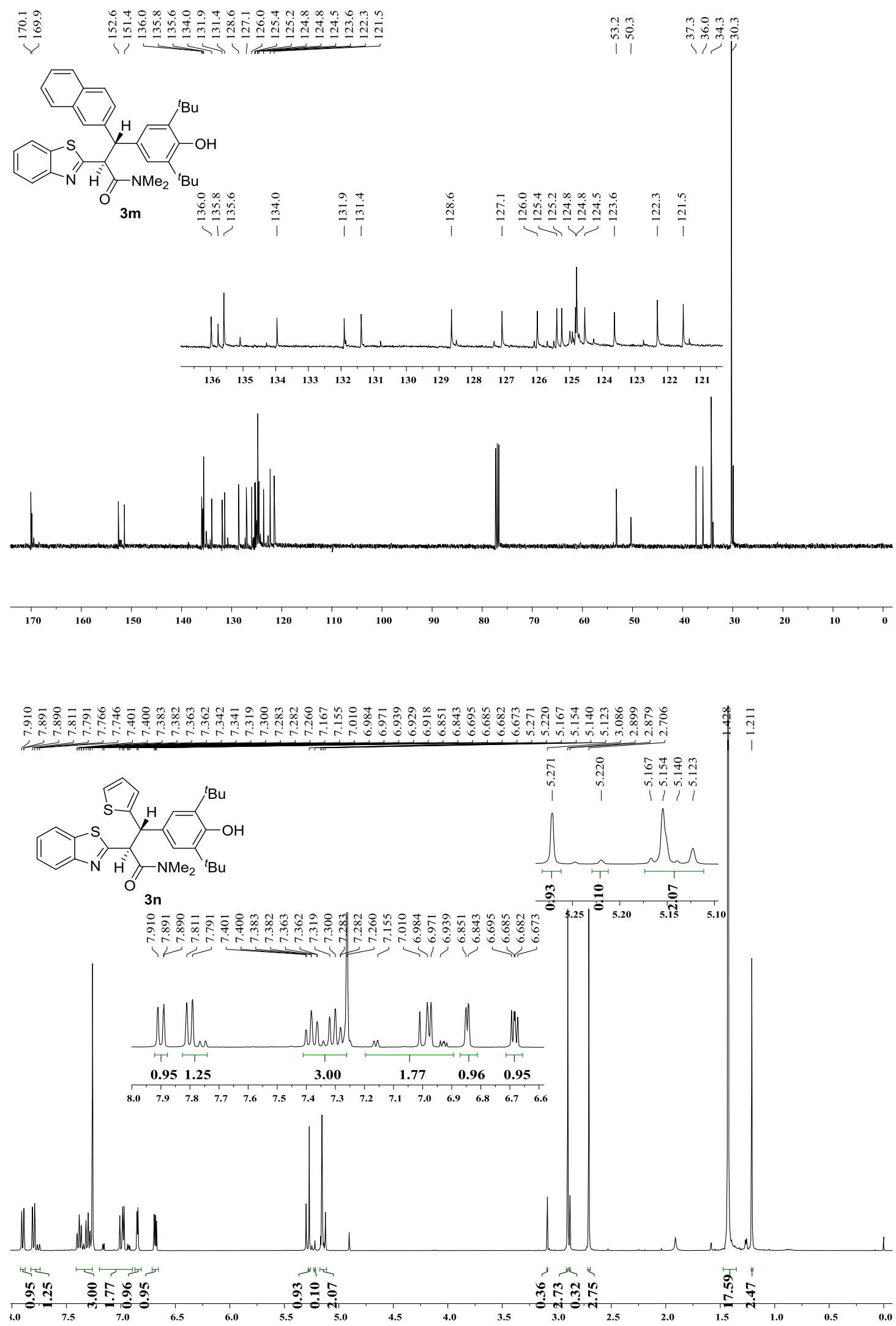

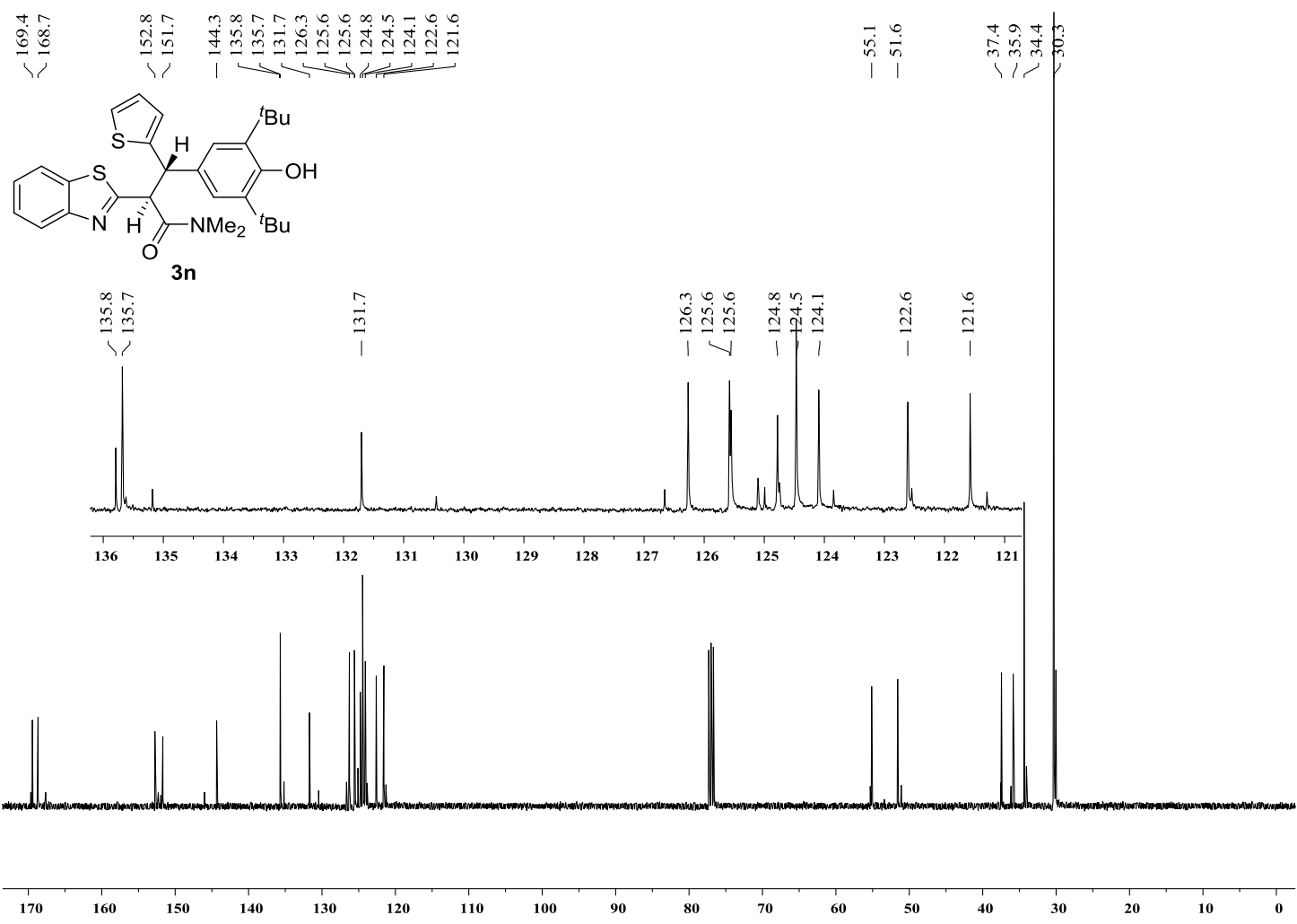

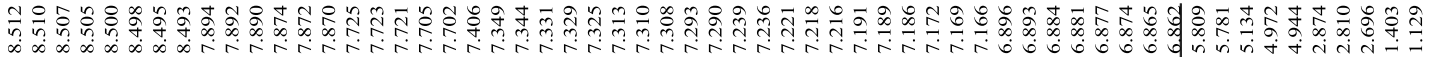
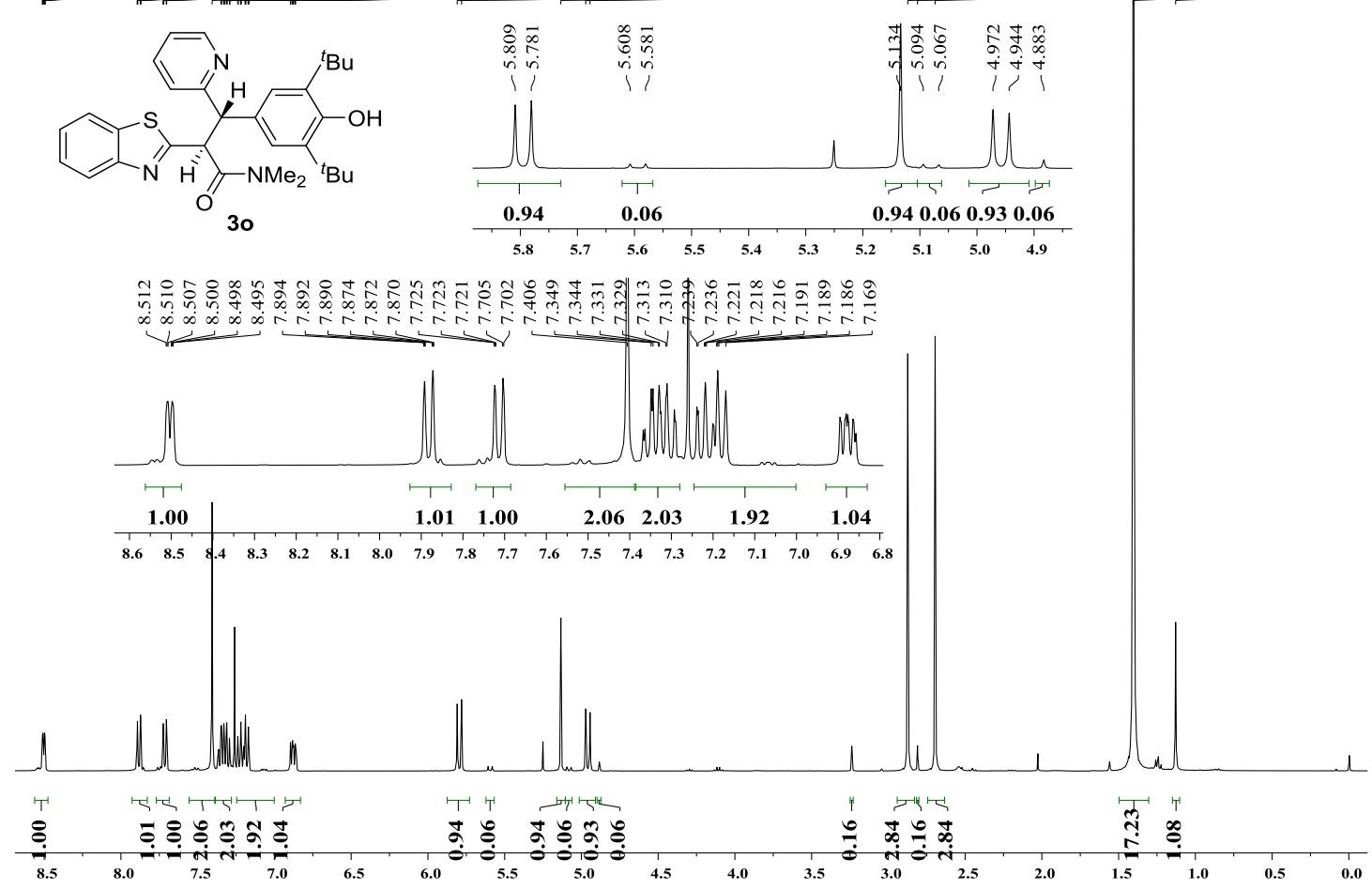

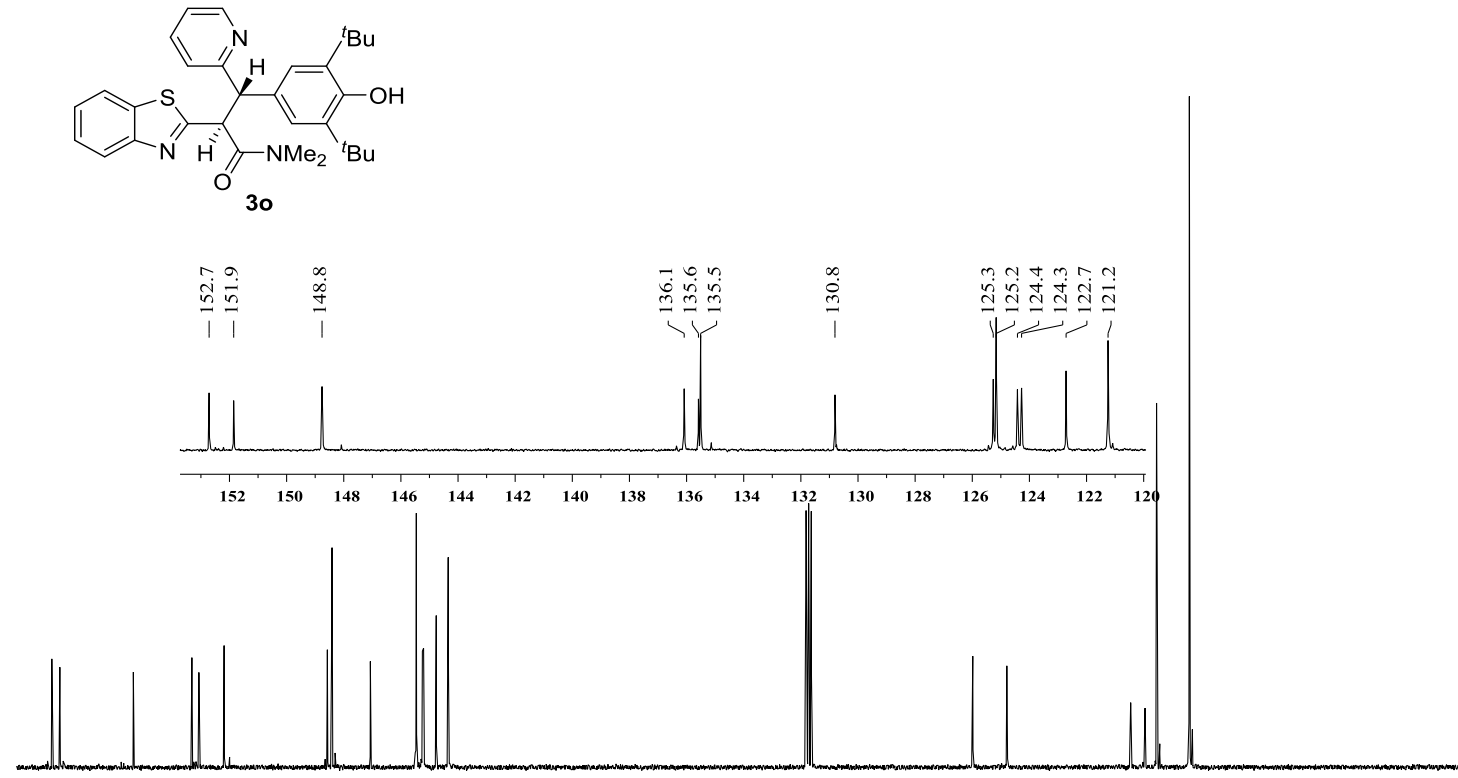

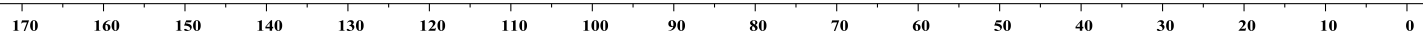

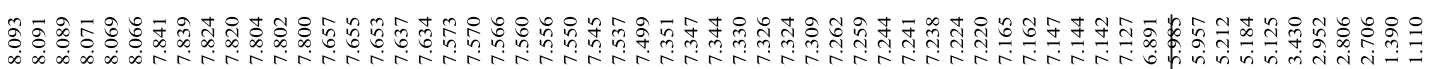
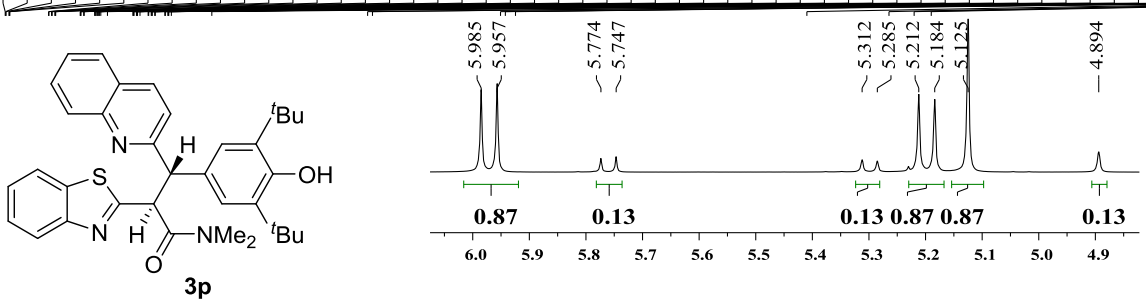

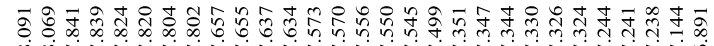

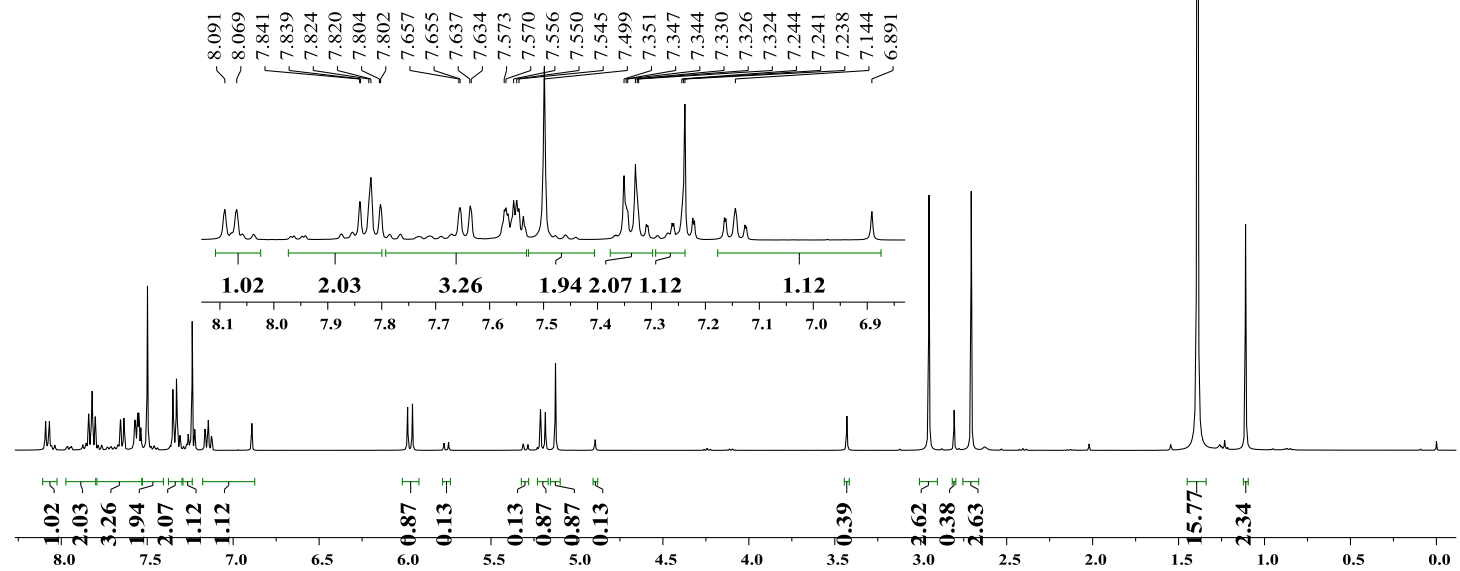



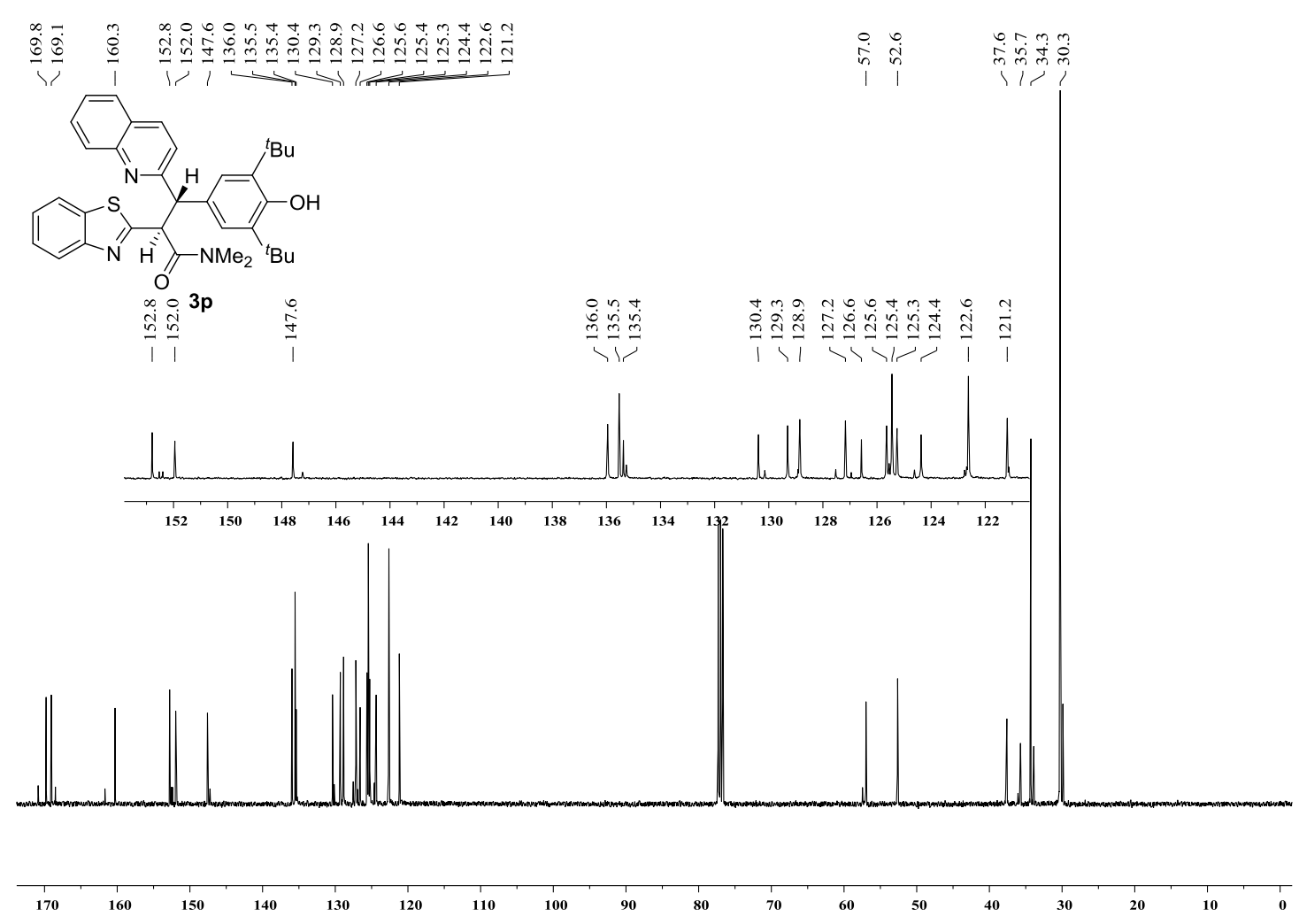

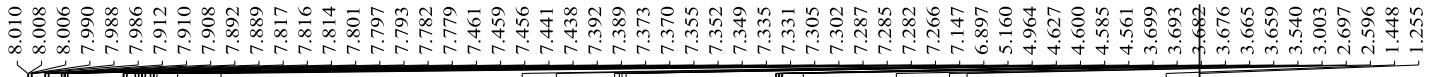

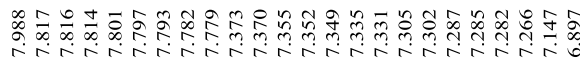
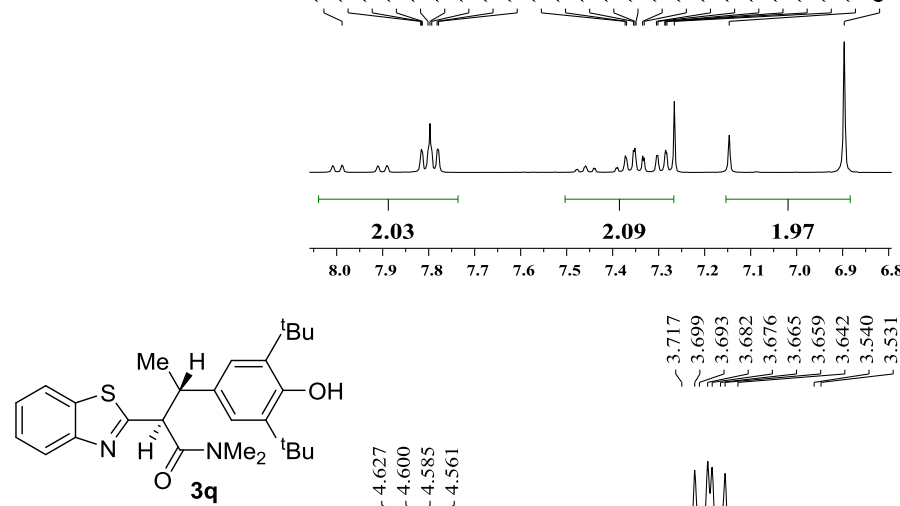

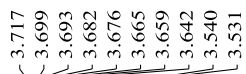

${ }_{3 q}$

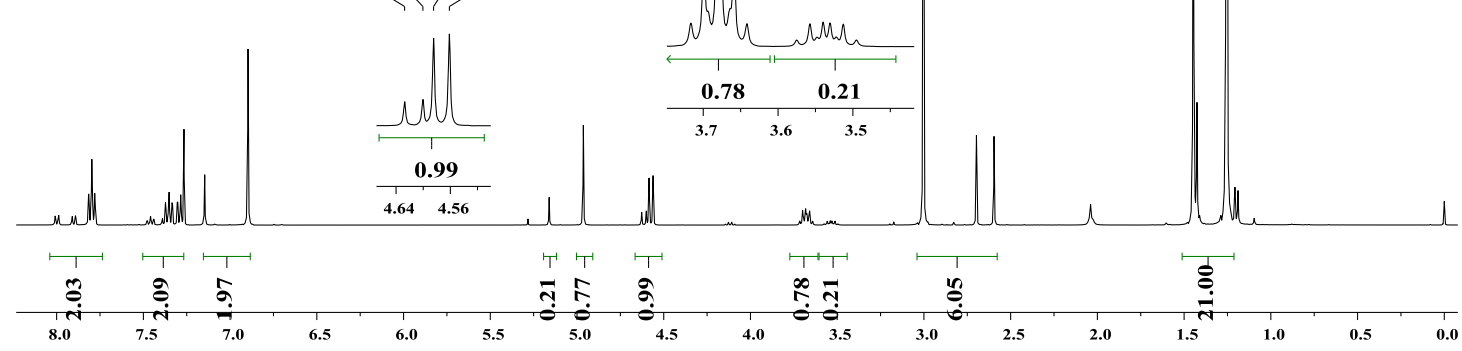




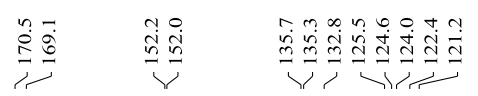
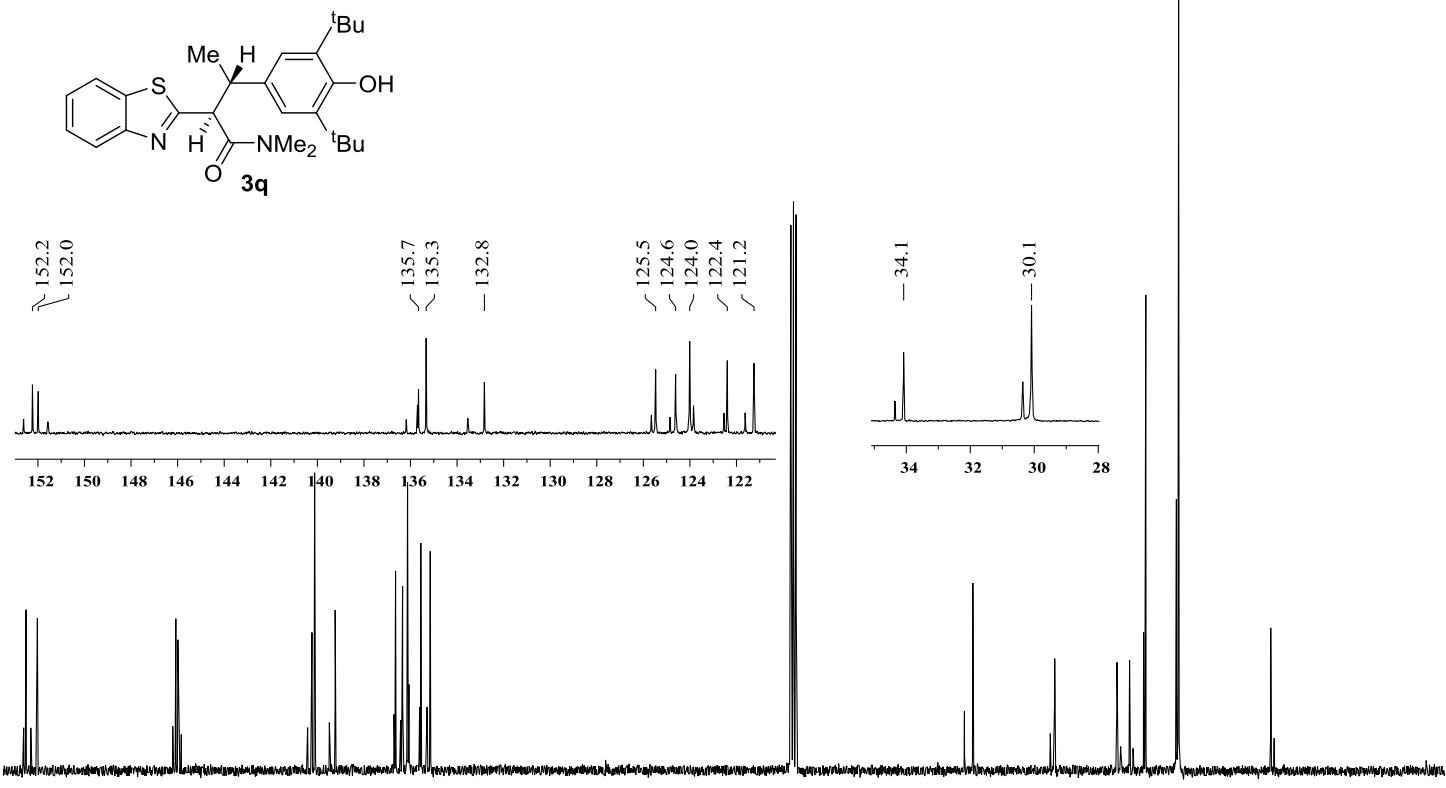

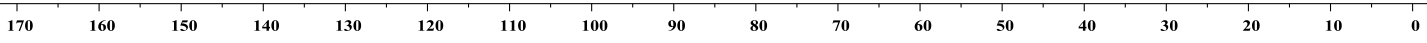

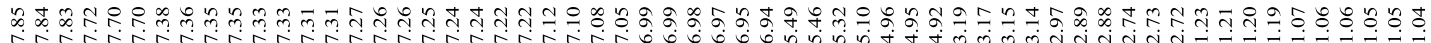
यPr<smiles>CC(=O)C(c1nc2ccccc2s1)[C@H](c1ccccc1)c1cc(C(C)C)c(O)c(C(C)C)c1</smiles>

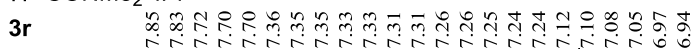
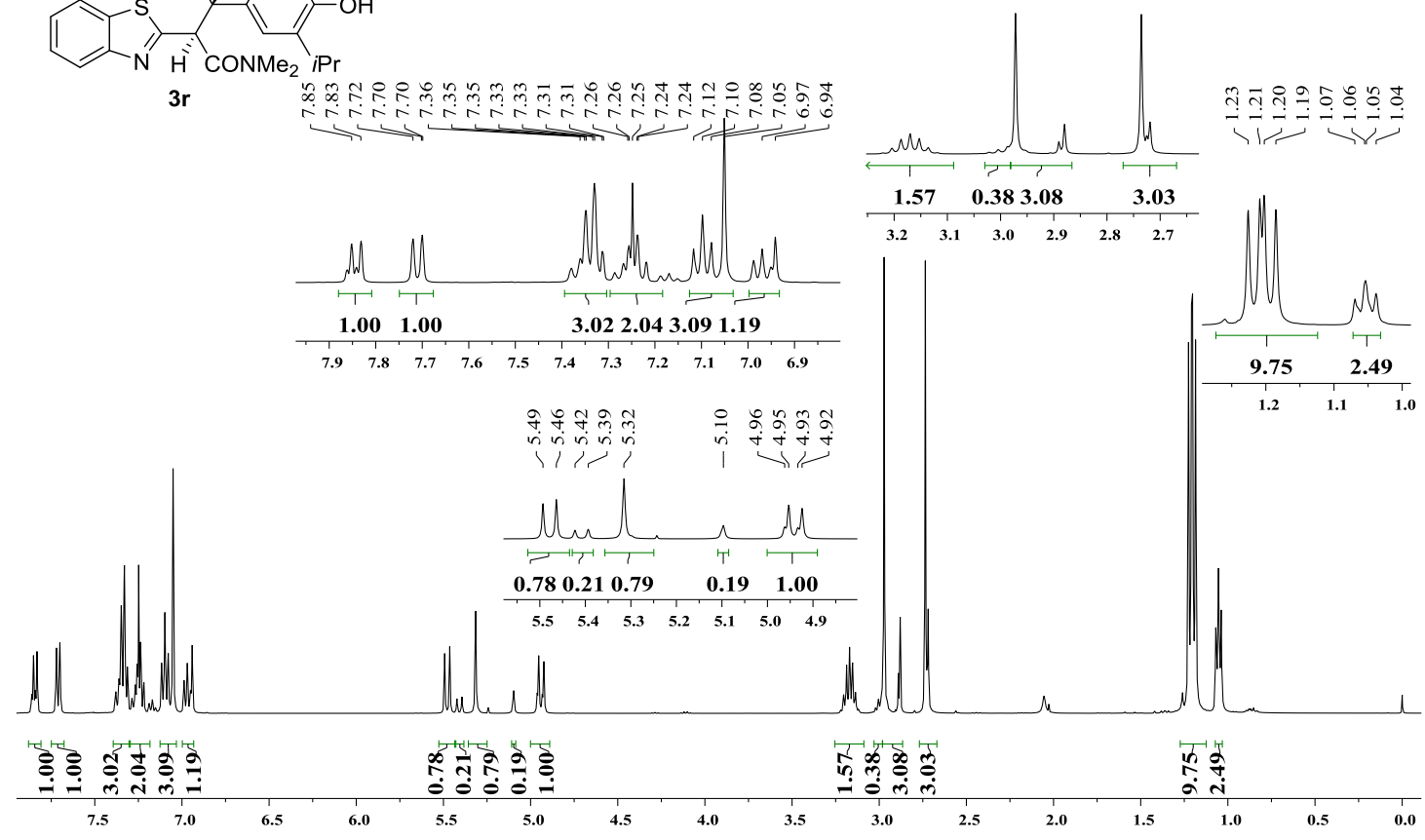


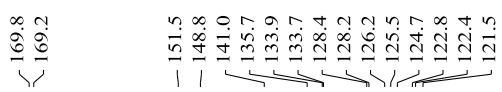

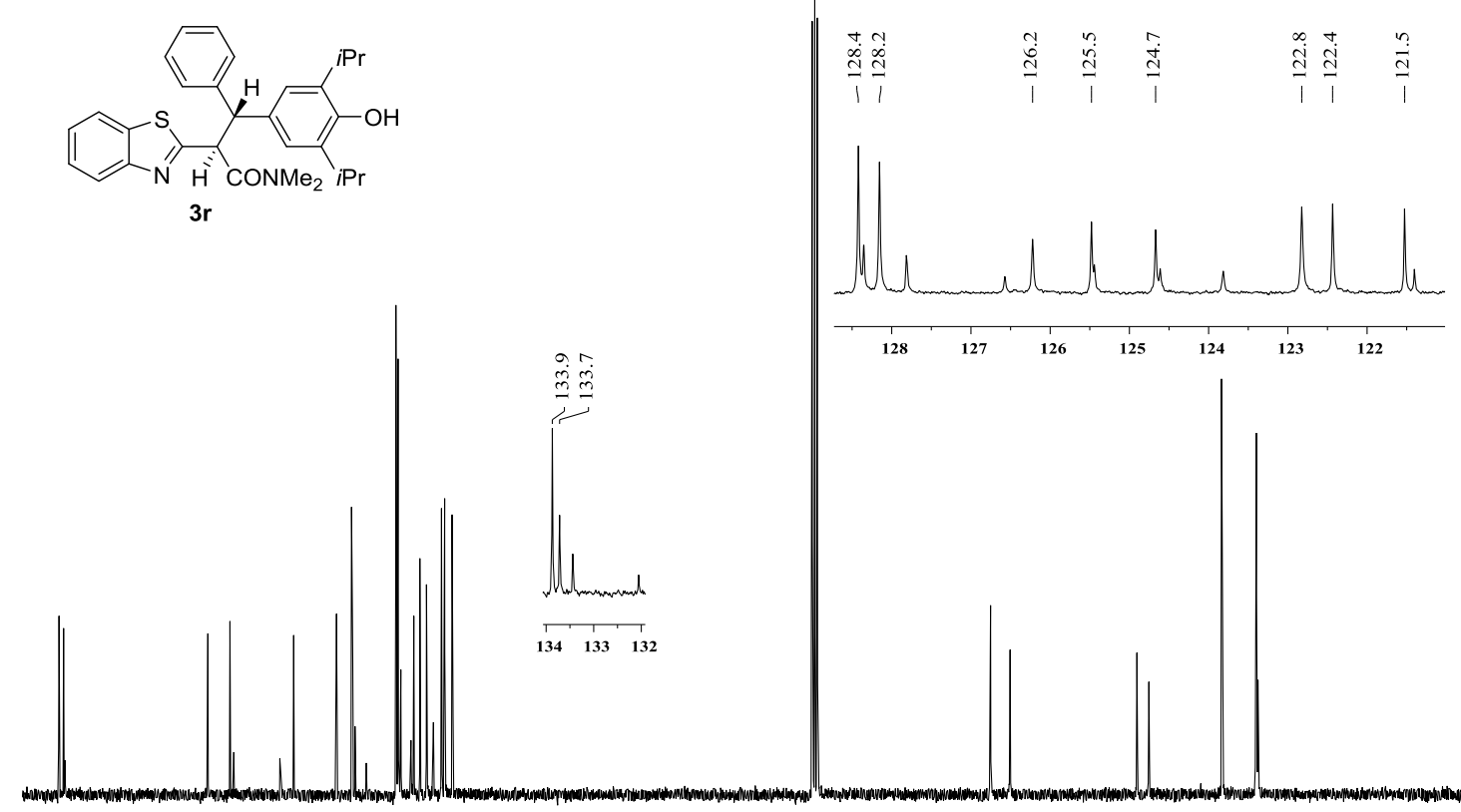

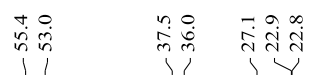

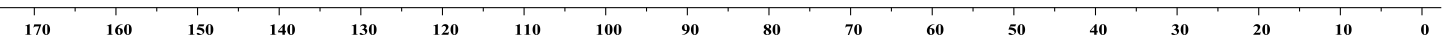

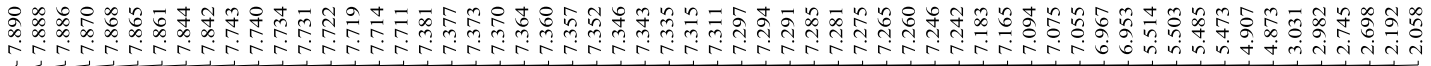<smiles>CC(=O)C(c1ccccc1)(c1cc(C)c(O)c(C)c1)c1nc2ccccc2s1</smiles>

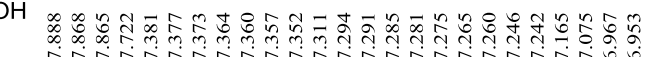
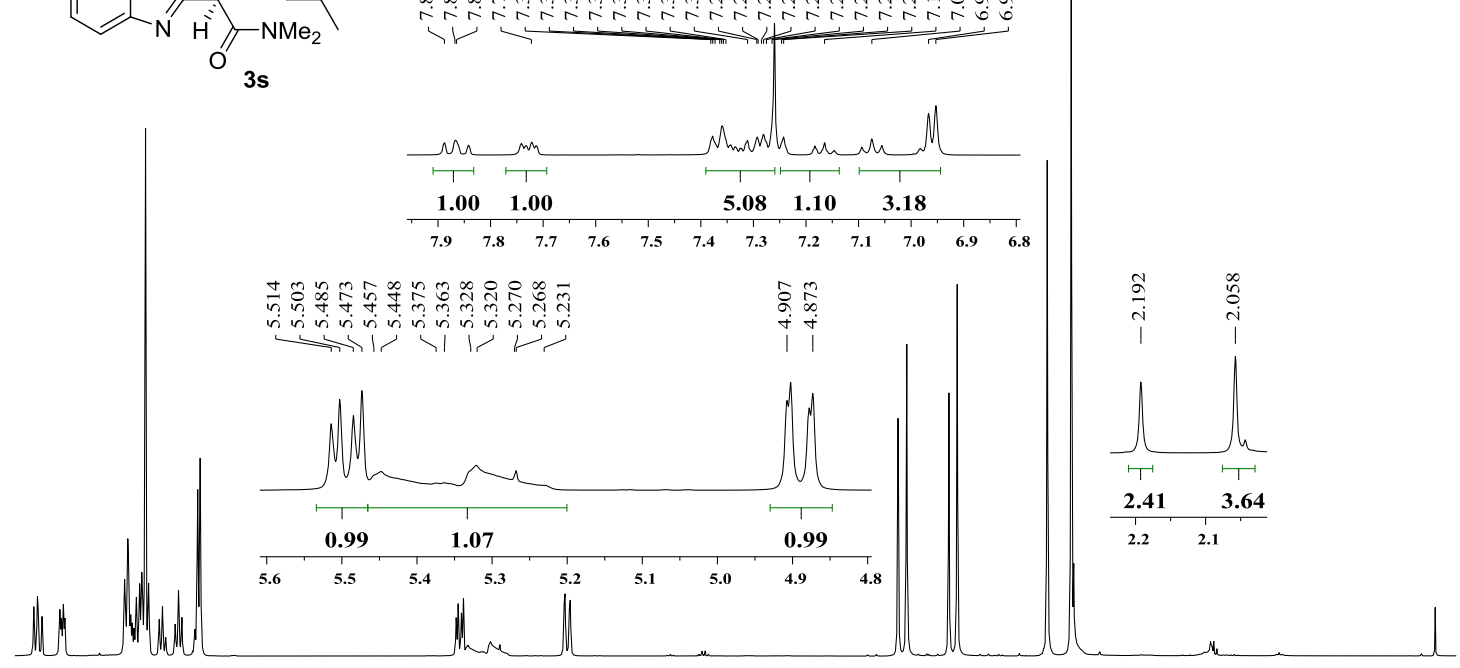

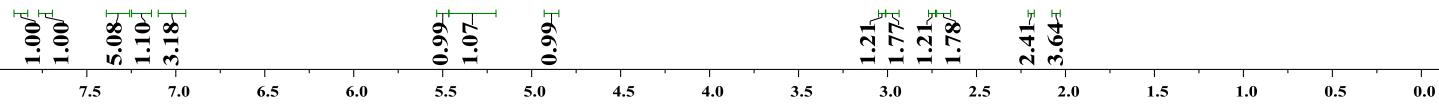




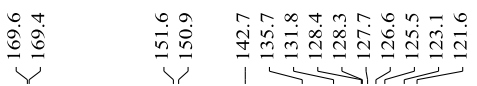<smiles>Cc1cc([C@H](c2ccccc2)[C@@H](C(N)=O)c2nc3ccccc3s2)cc(C)c1O</smiles>

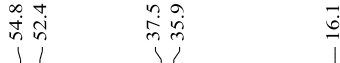

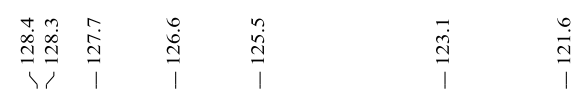

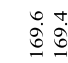
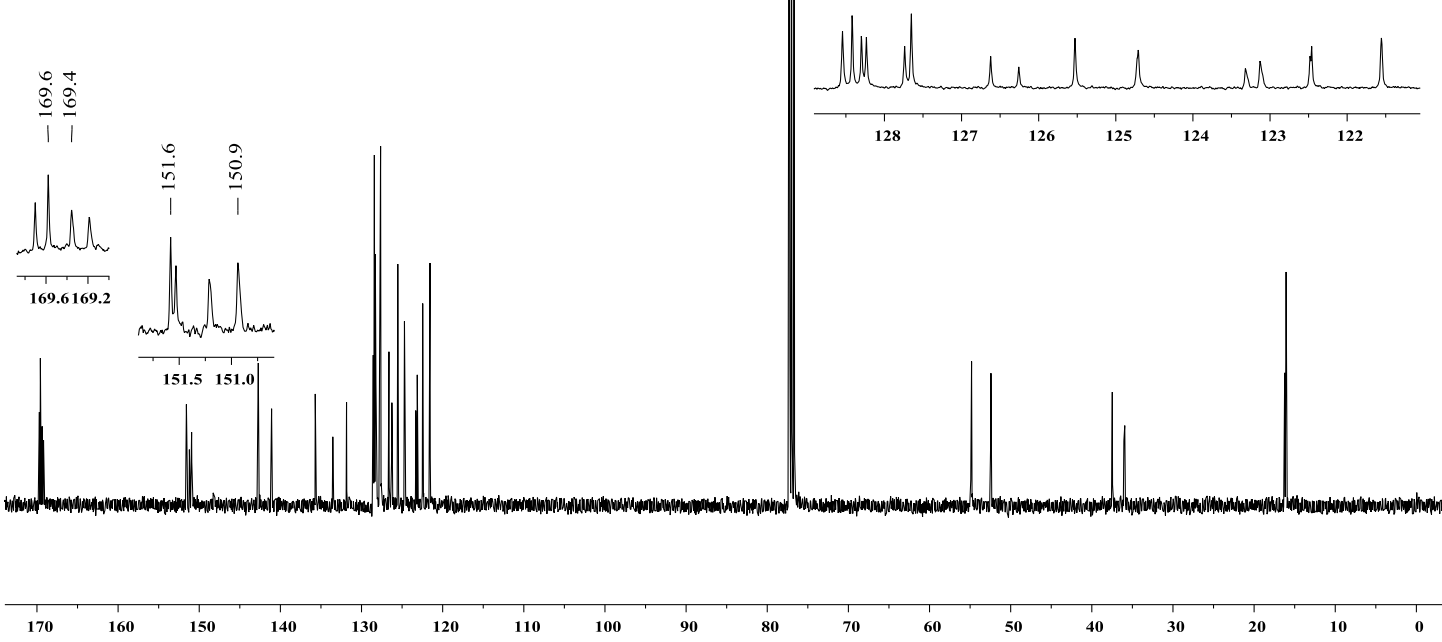

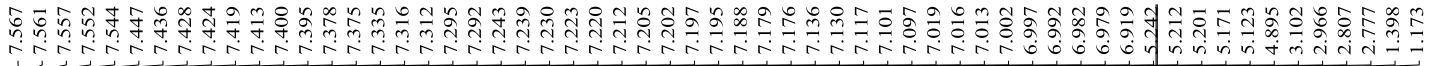
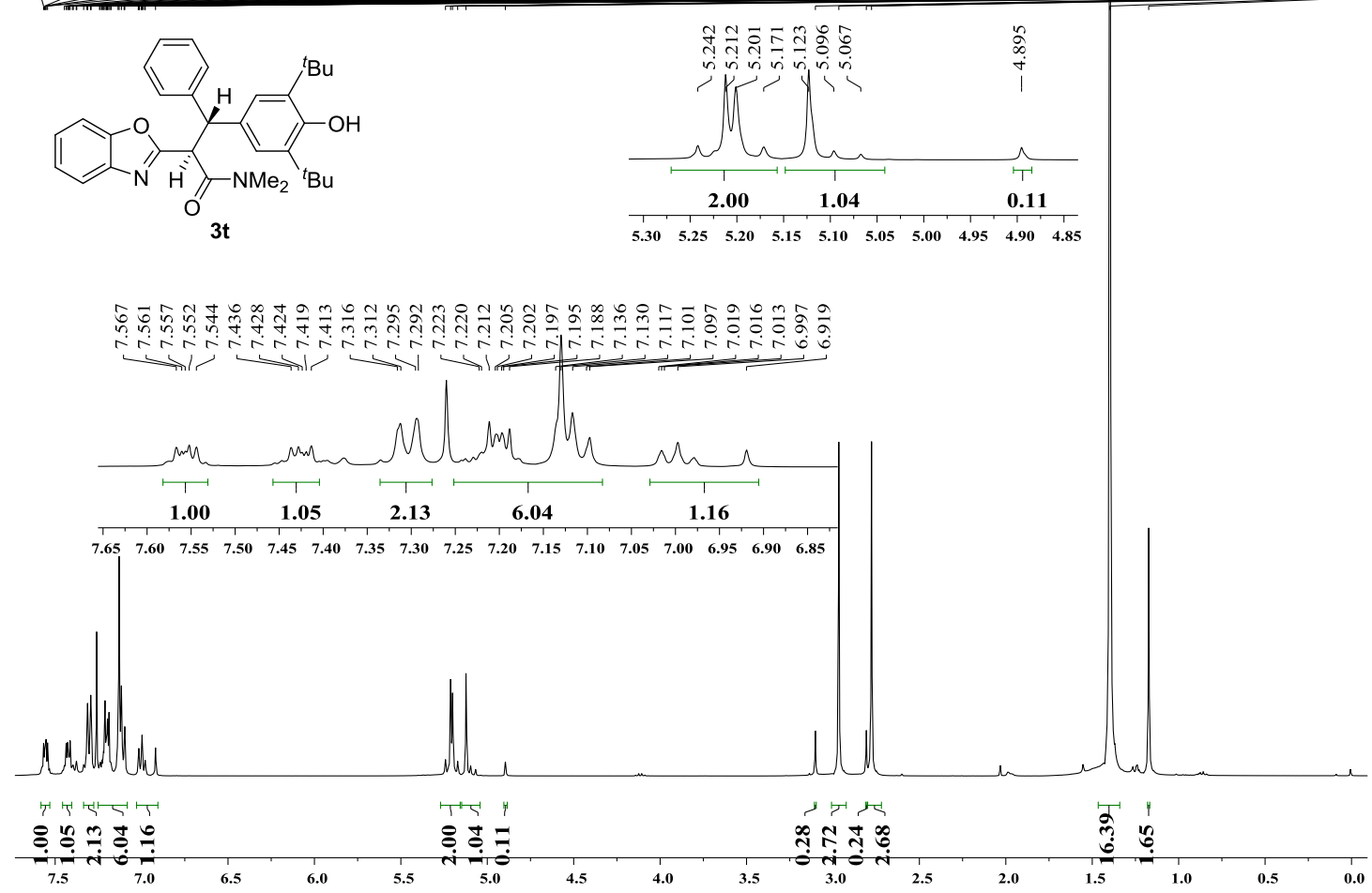
<smiles>CC(=O)[C@@H](c1nc2ccccc2o1)[C@@H](c1ccccc1)c1cc([13CH3])c(O)c(C(C)(C)C)c1</smiles>

$3 t$

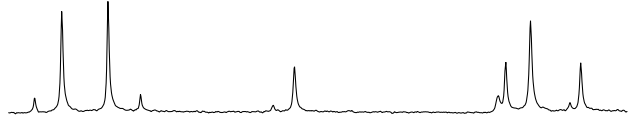

$\begin{array}{llllllllll}128.5 & 128.0 & 127.5 & 127.0 & 126.5 & 126.0 & 125.5 & 125.0 & 124.5 & 124.0\end{array}$

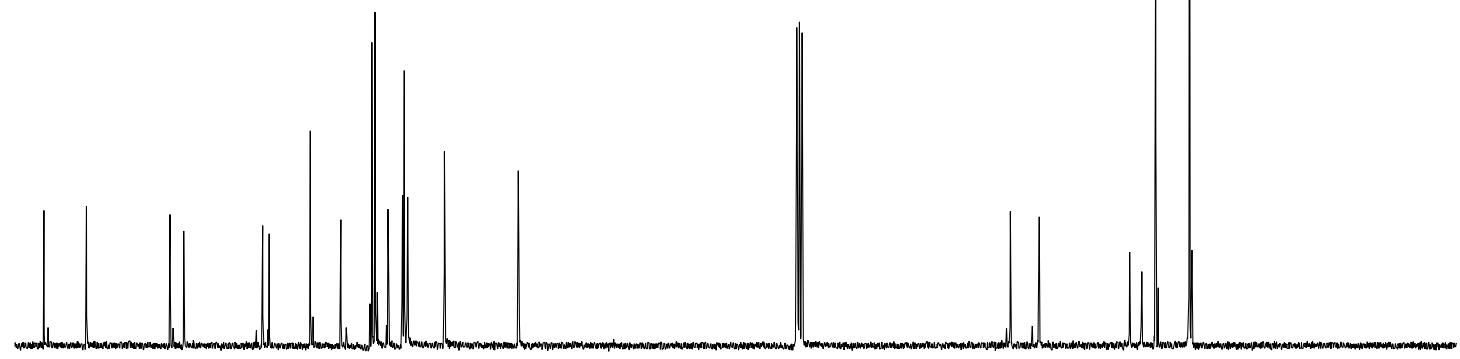

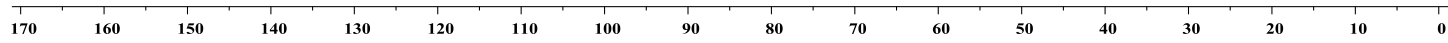

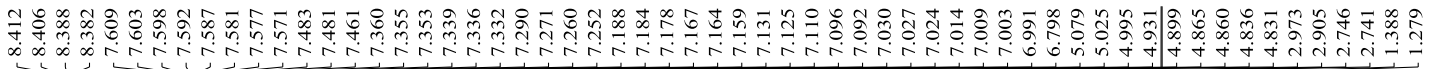
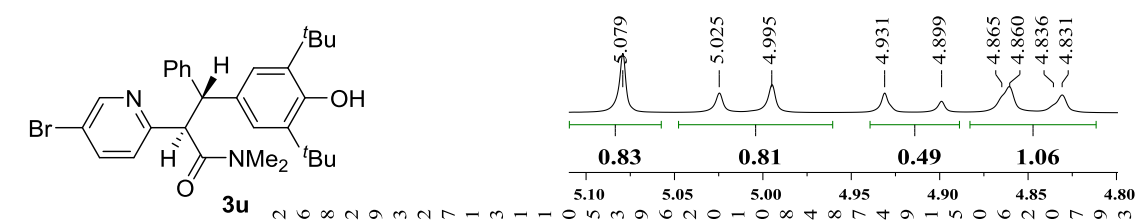

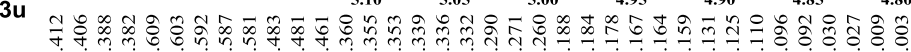

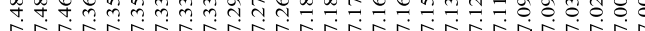

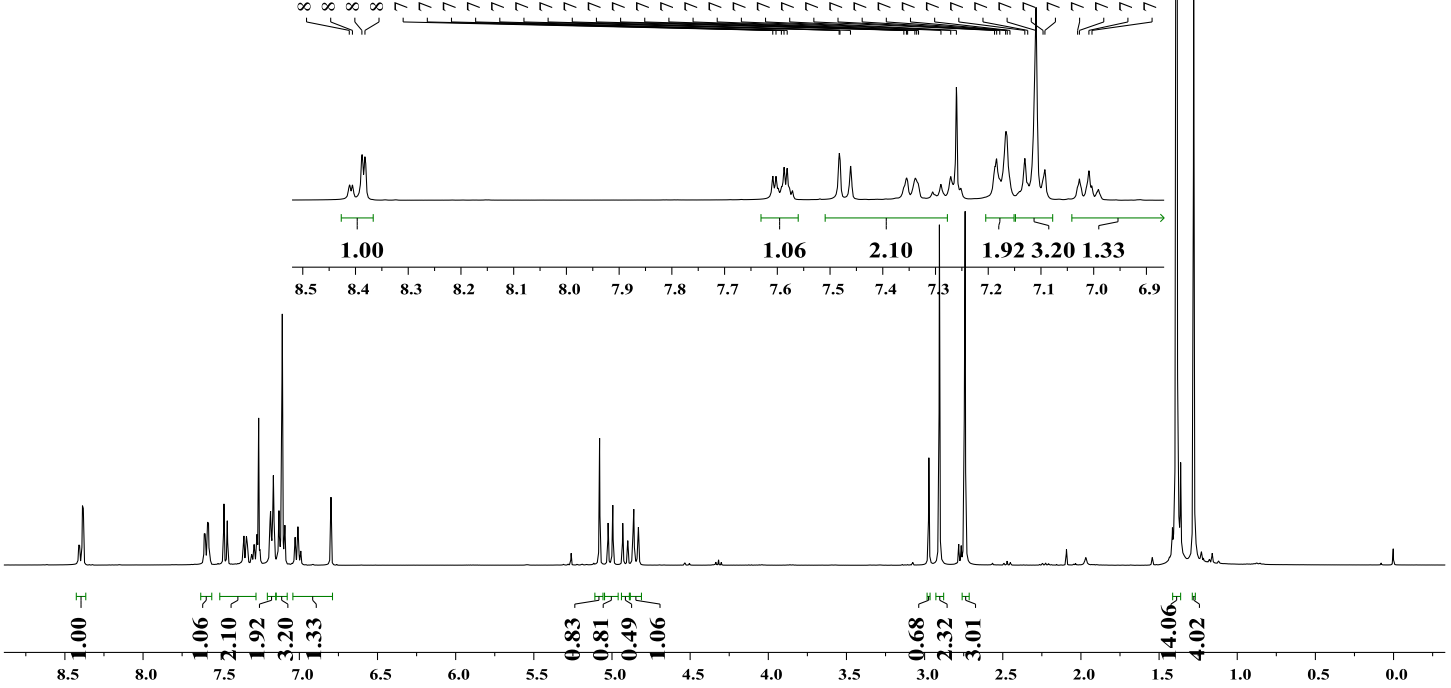




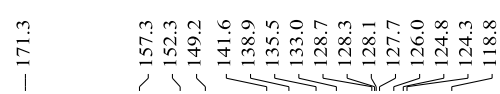

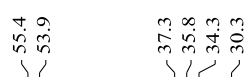

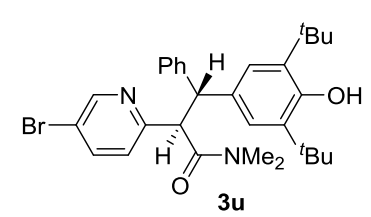

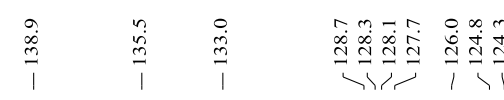

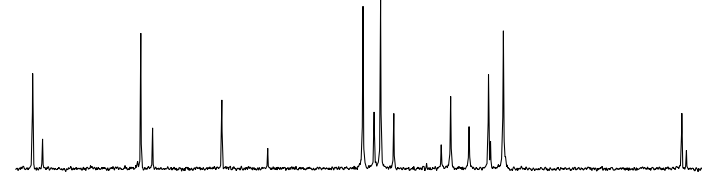

$\begin{array}{llllllllll}138 & 136 & 134 & 132 & 130 & 128 & 126 & 124 & 122 & 120\end{array}$
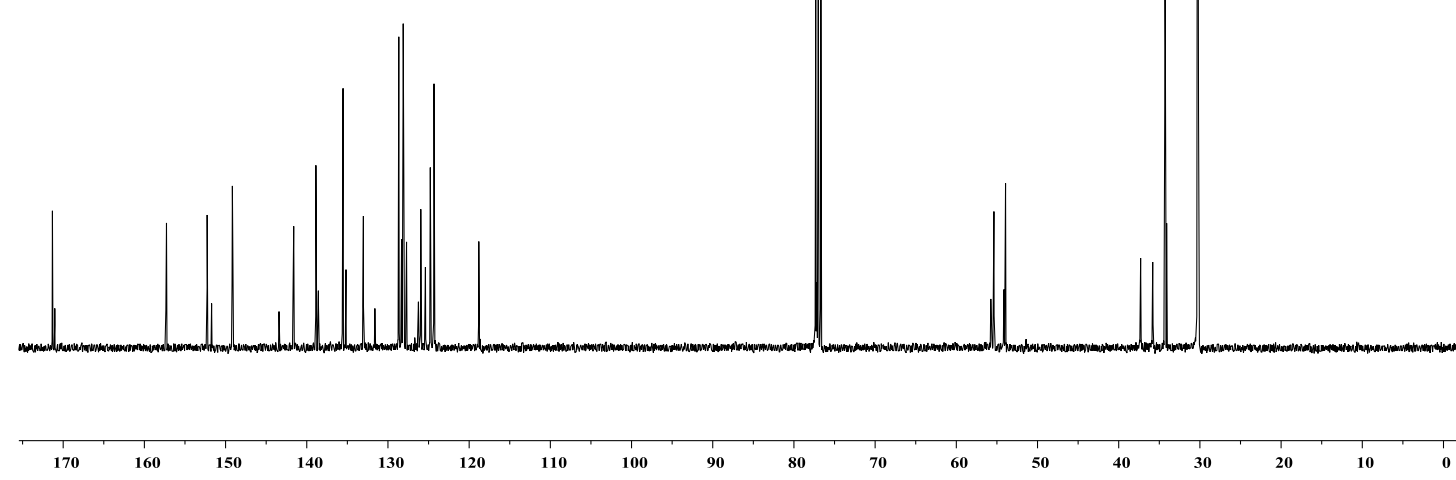

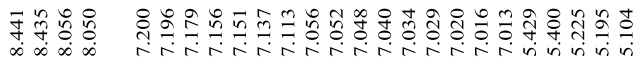

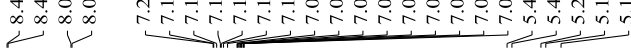

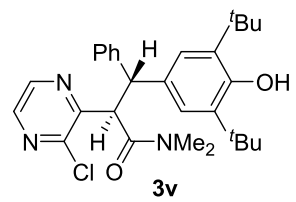

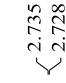
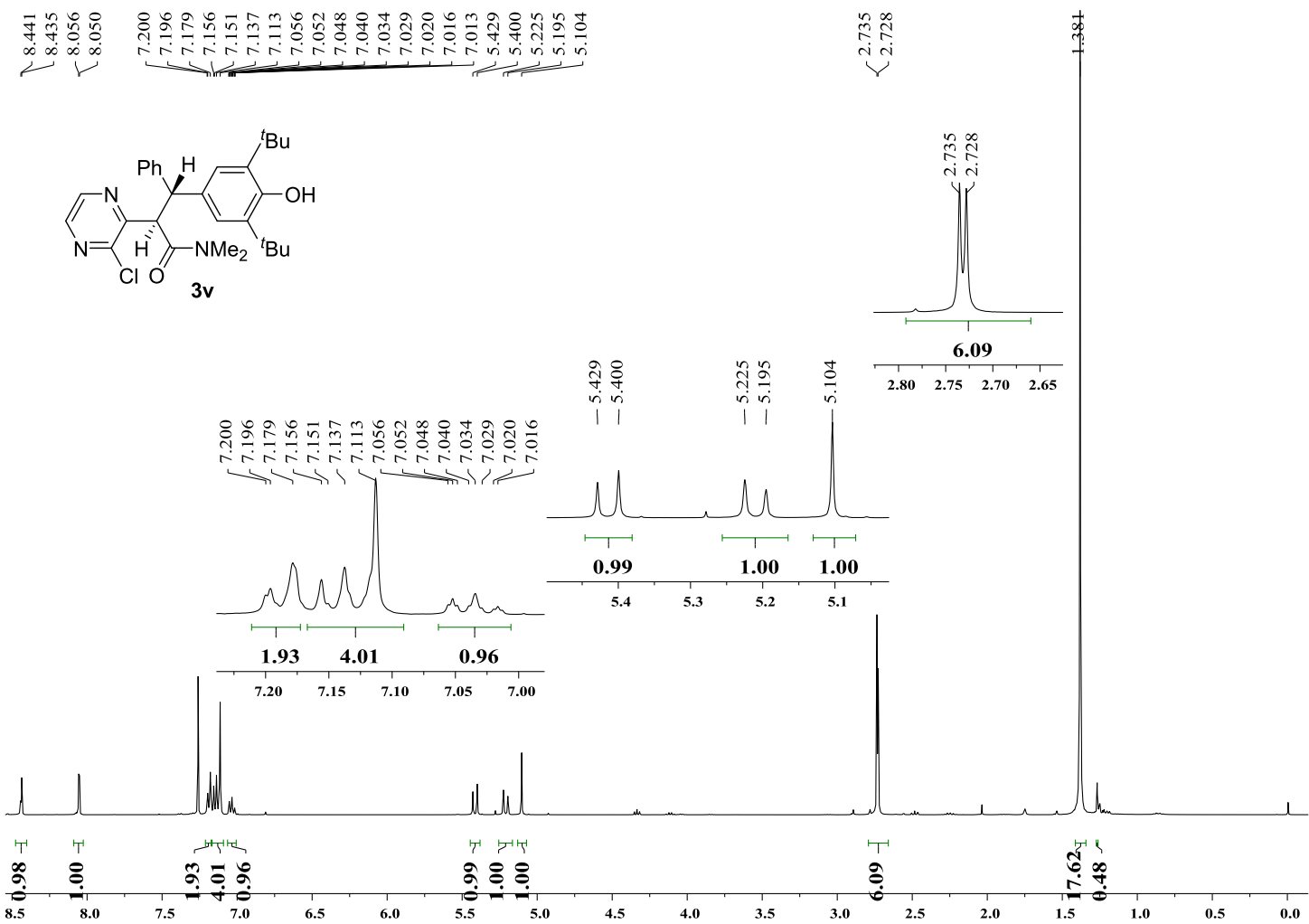

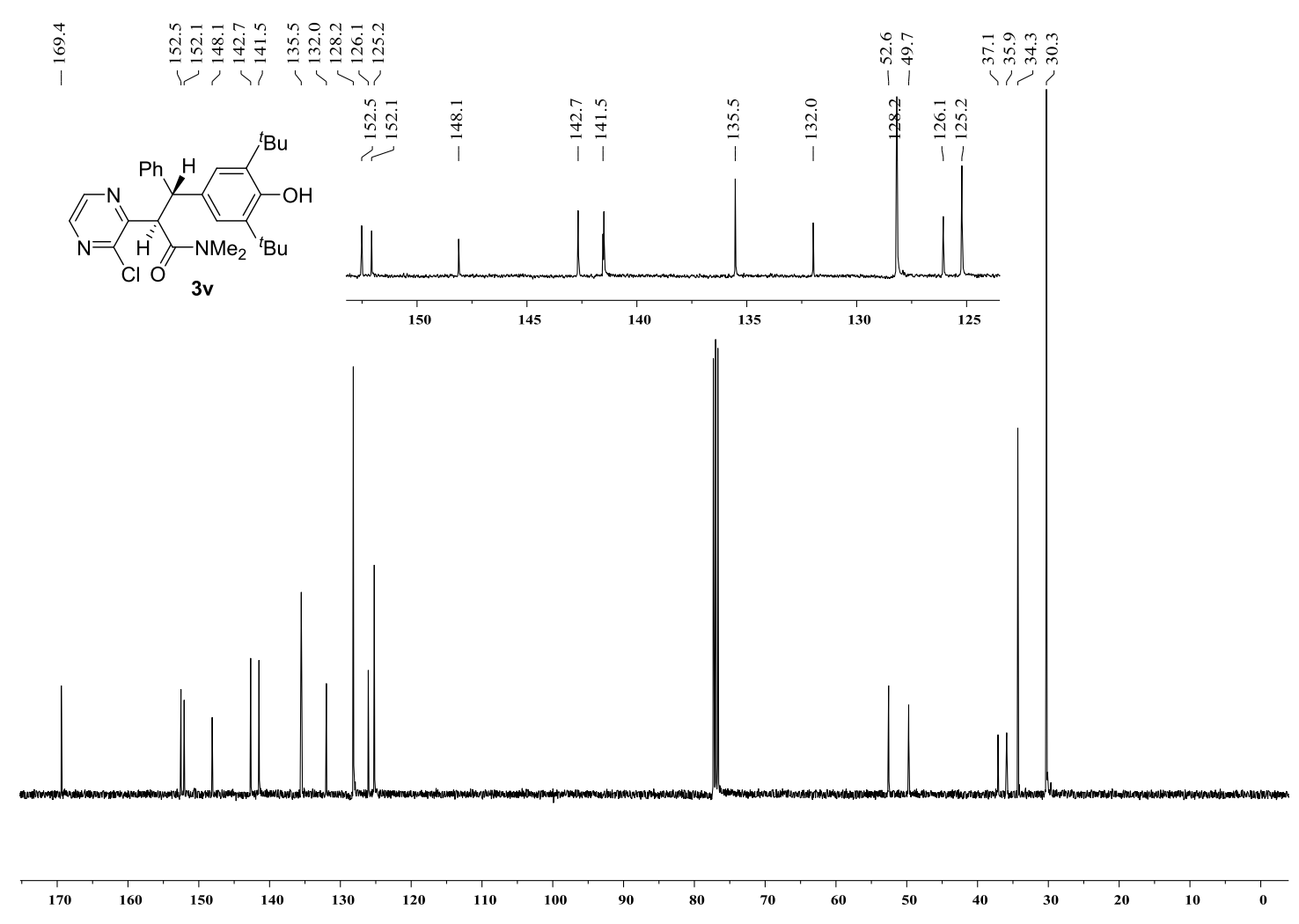

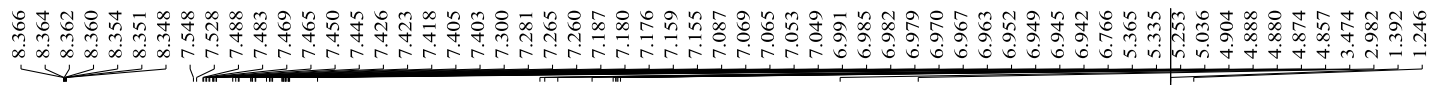

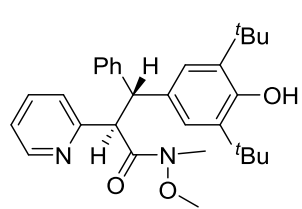

$3 w$
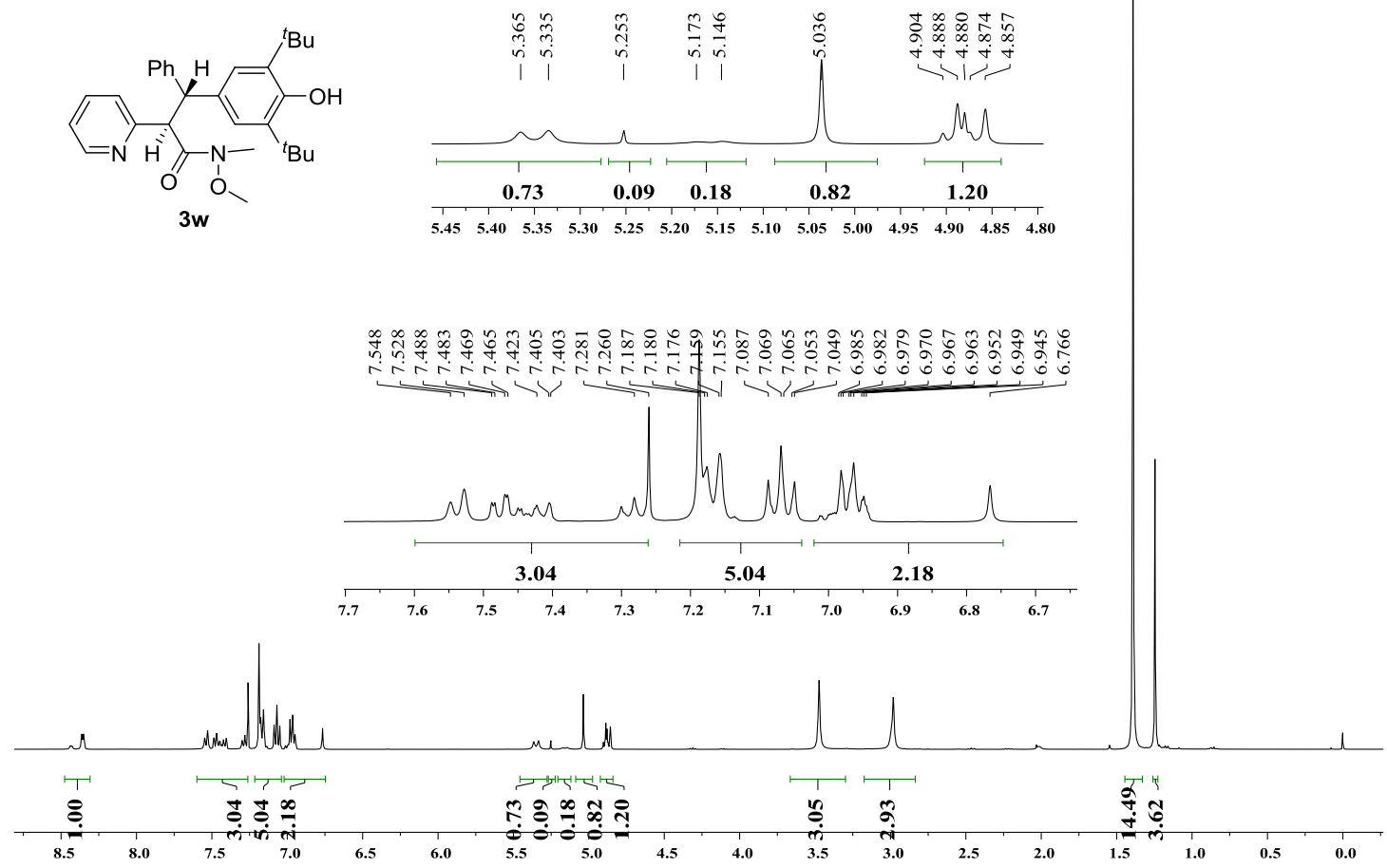

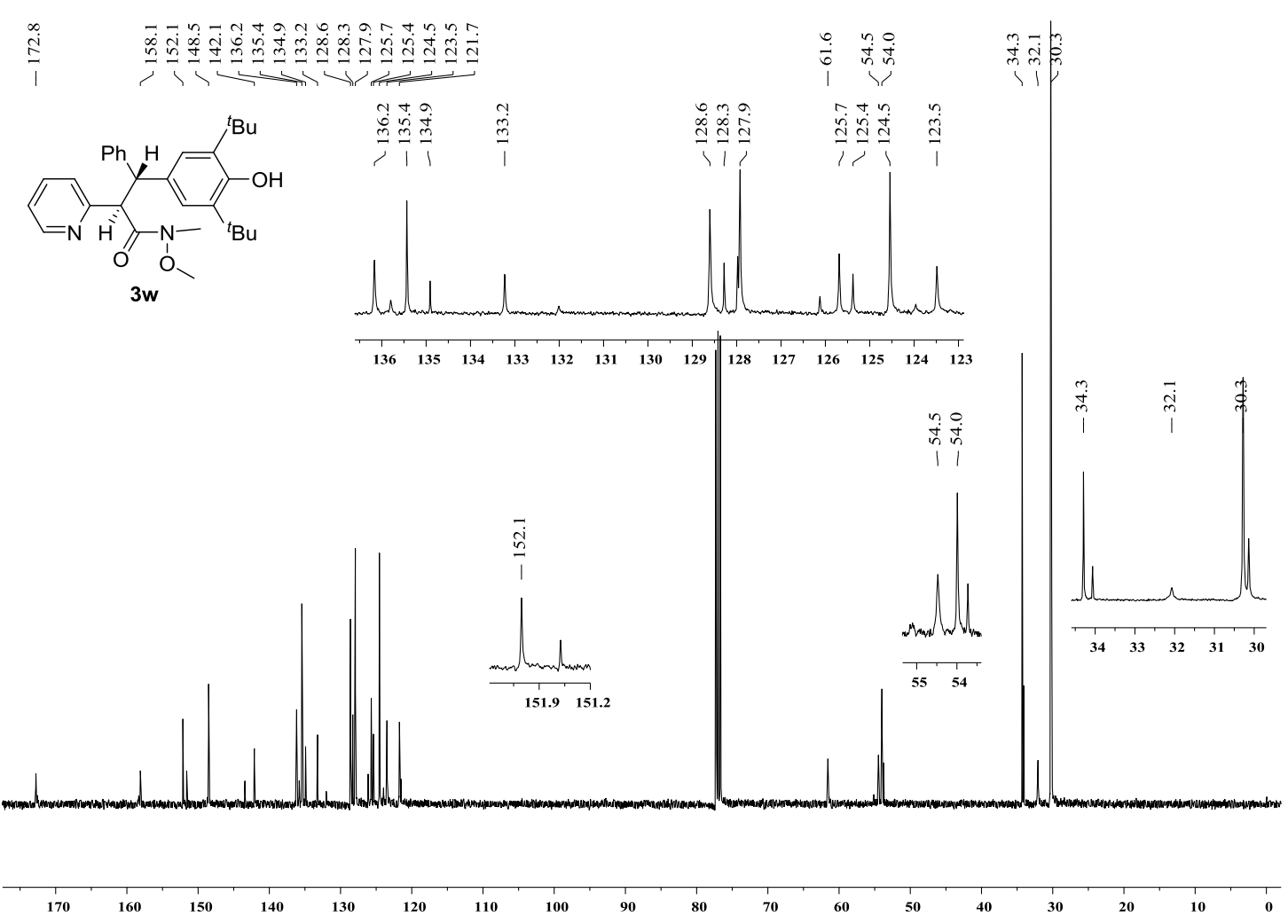

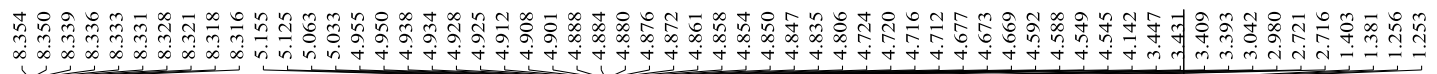

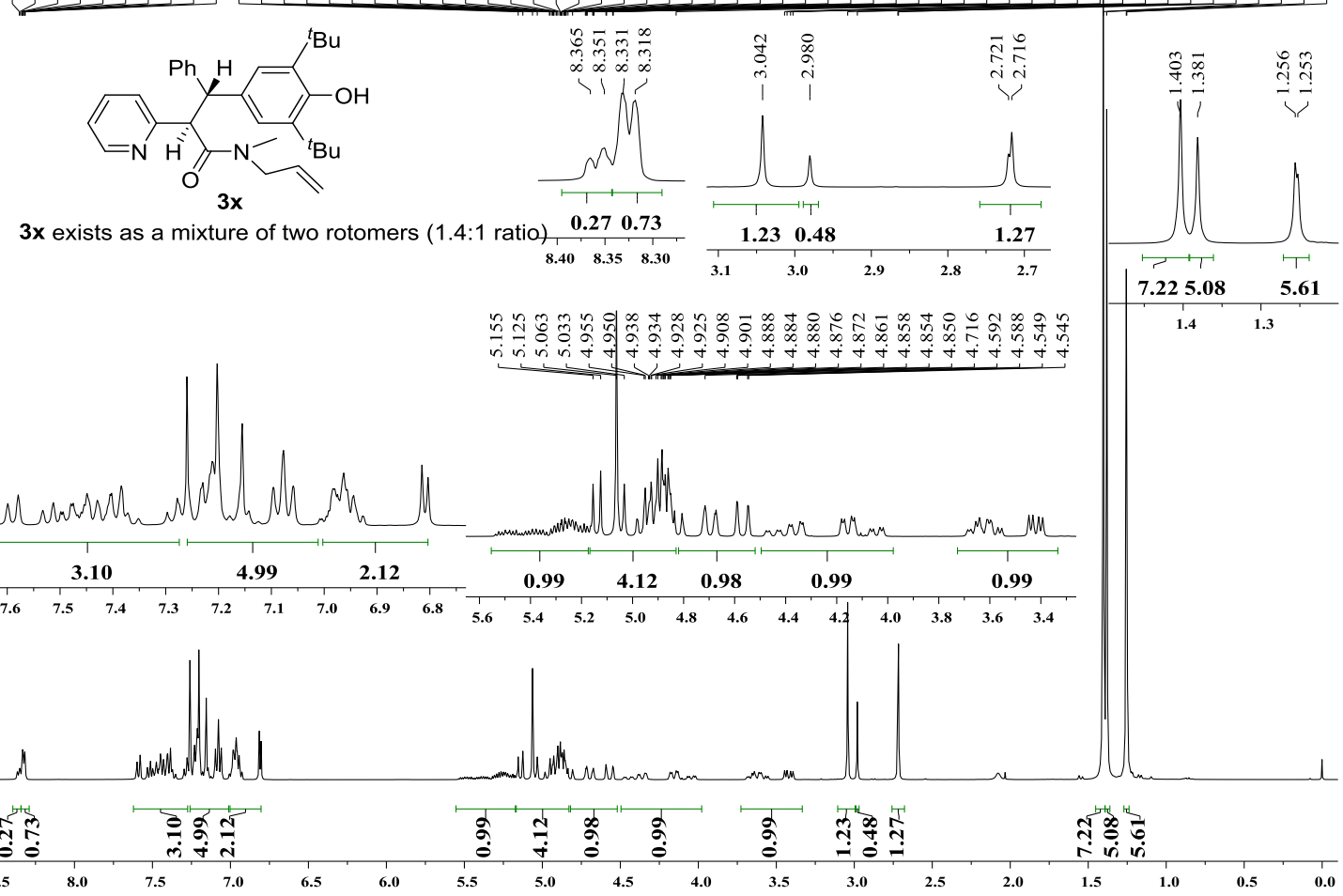




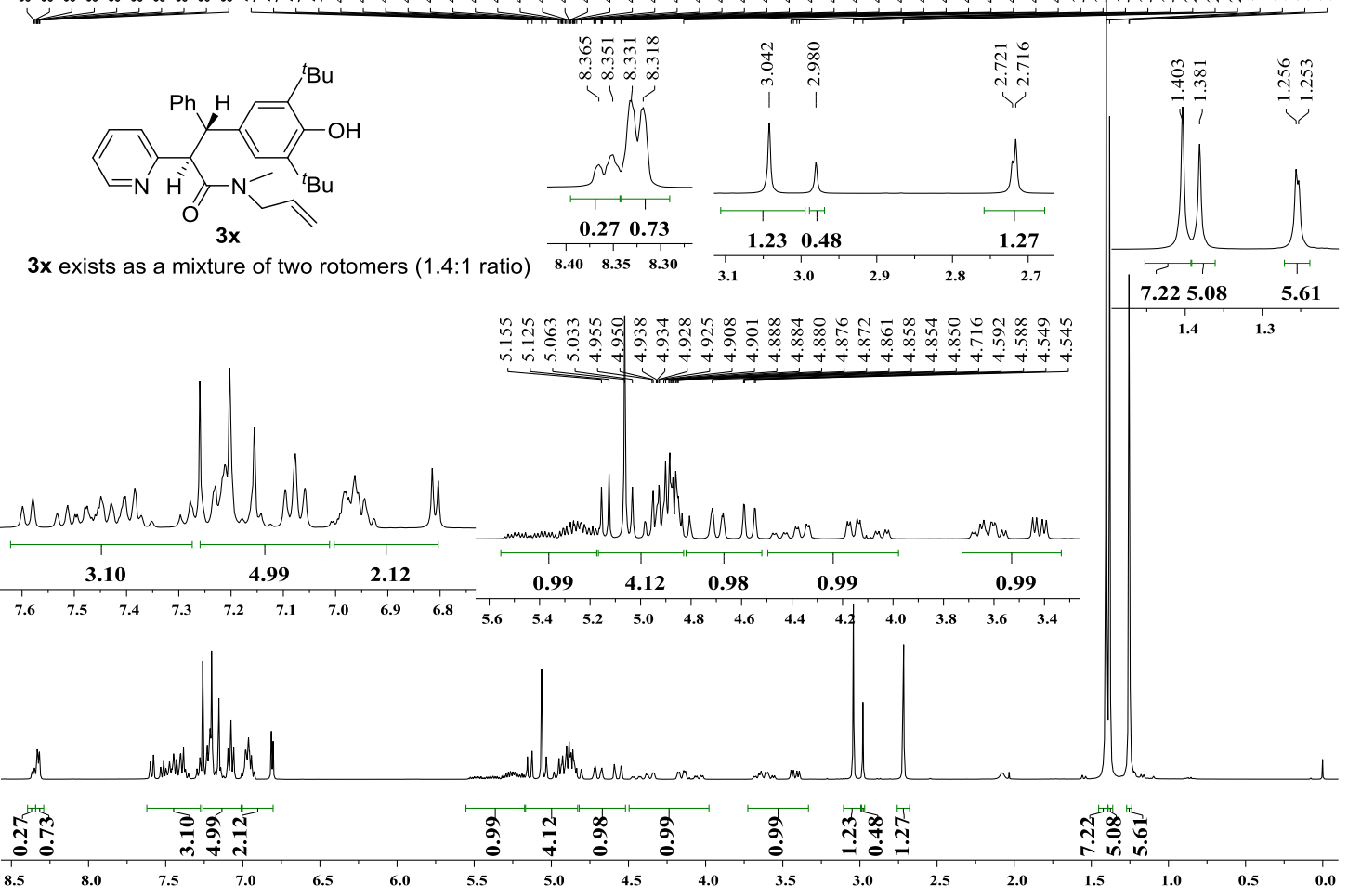

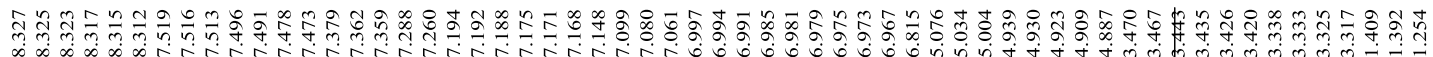

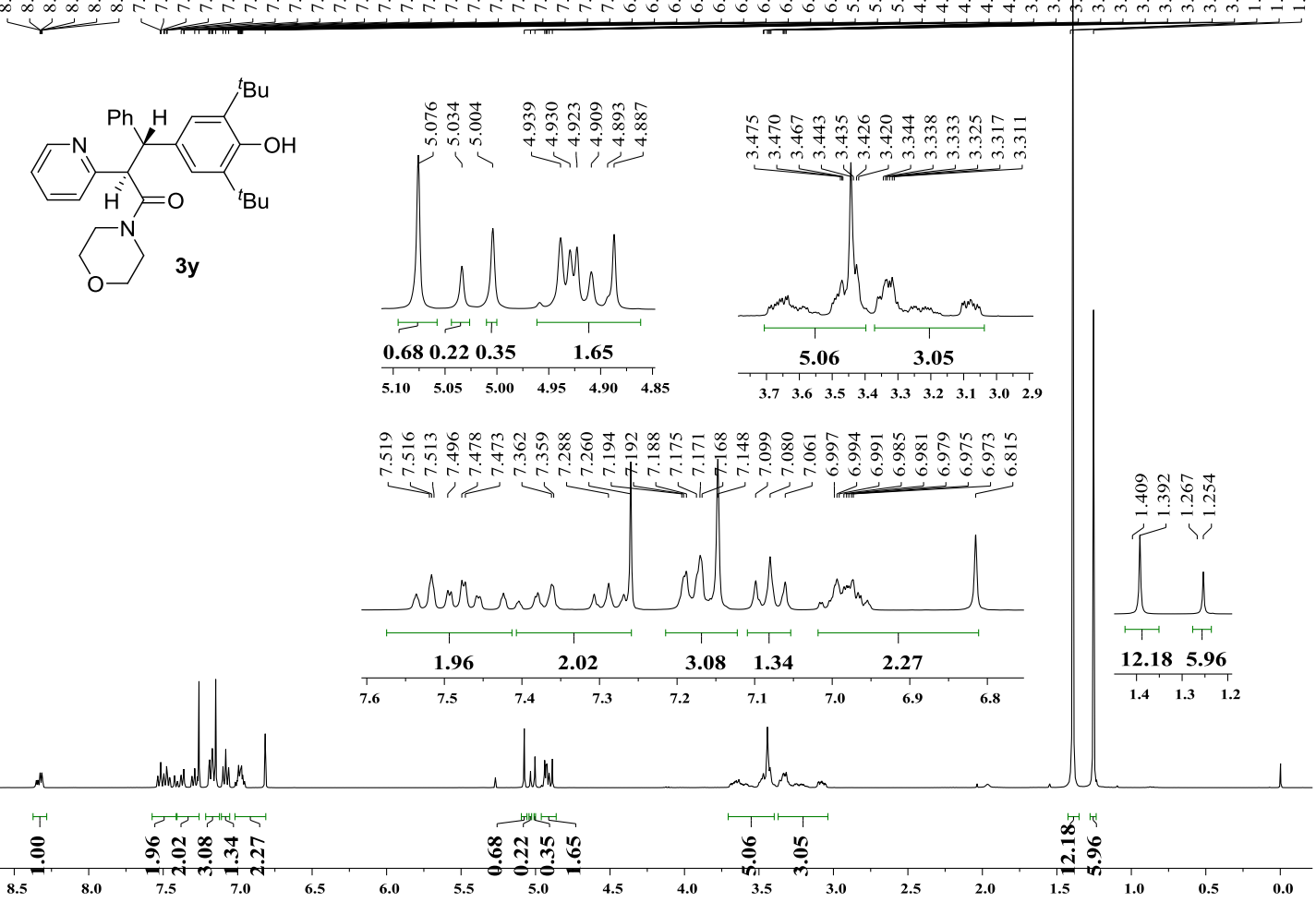




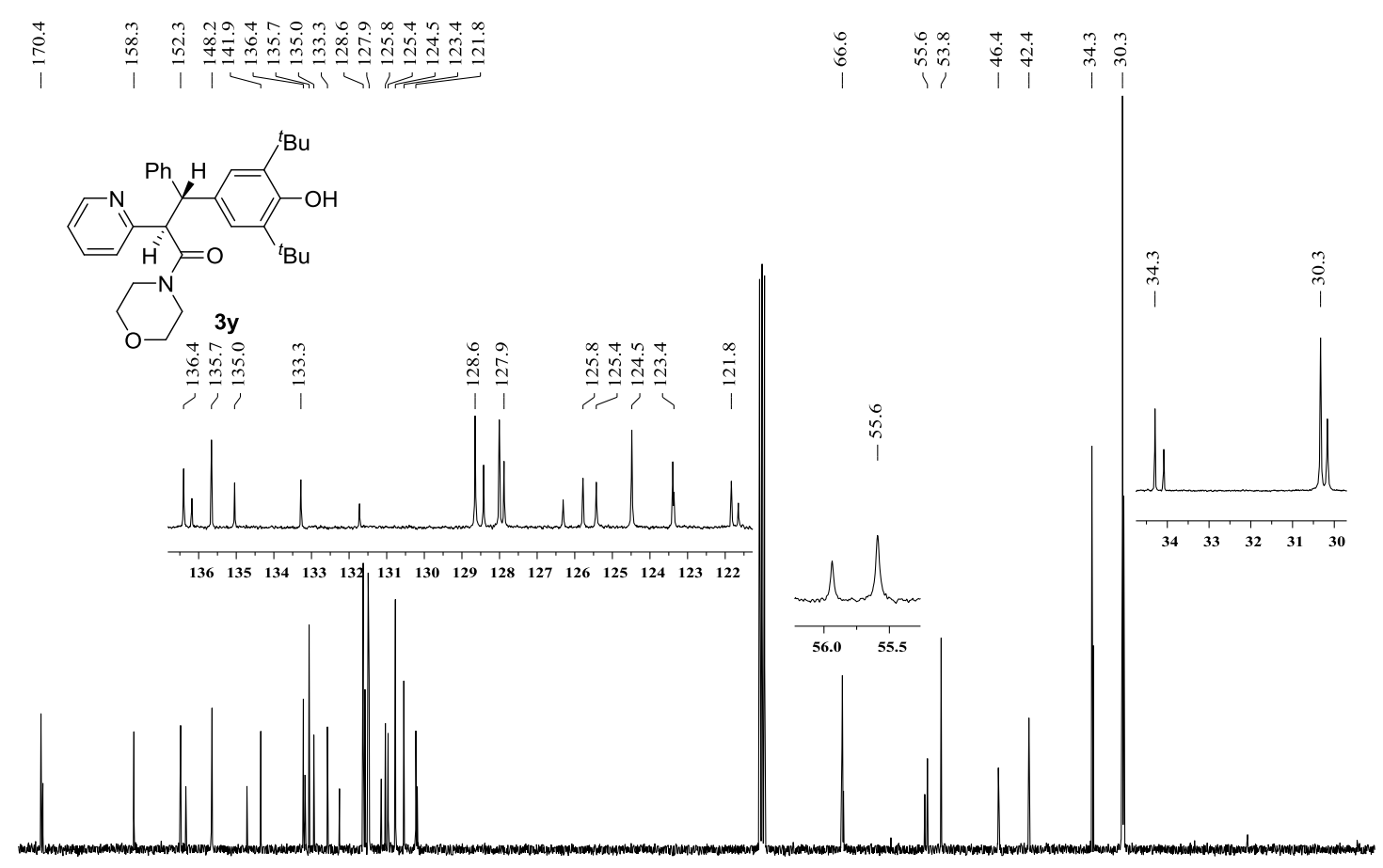

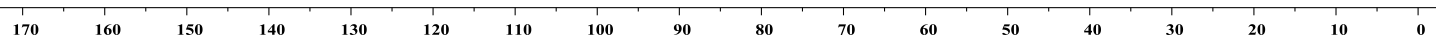

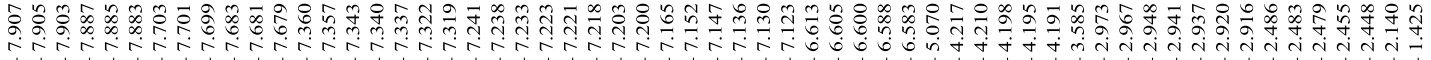
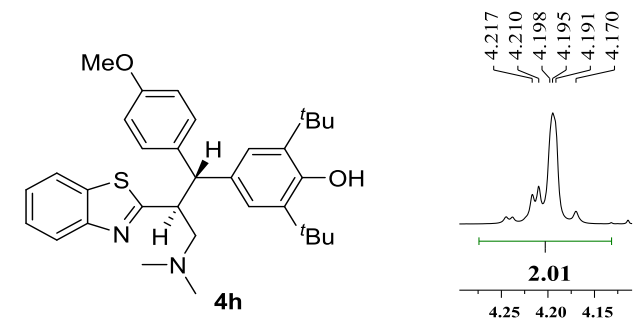

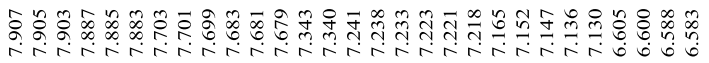

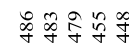

ivivi
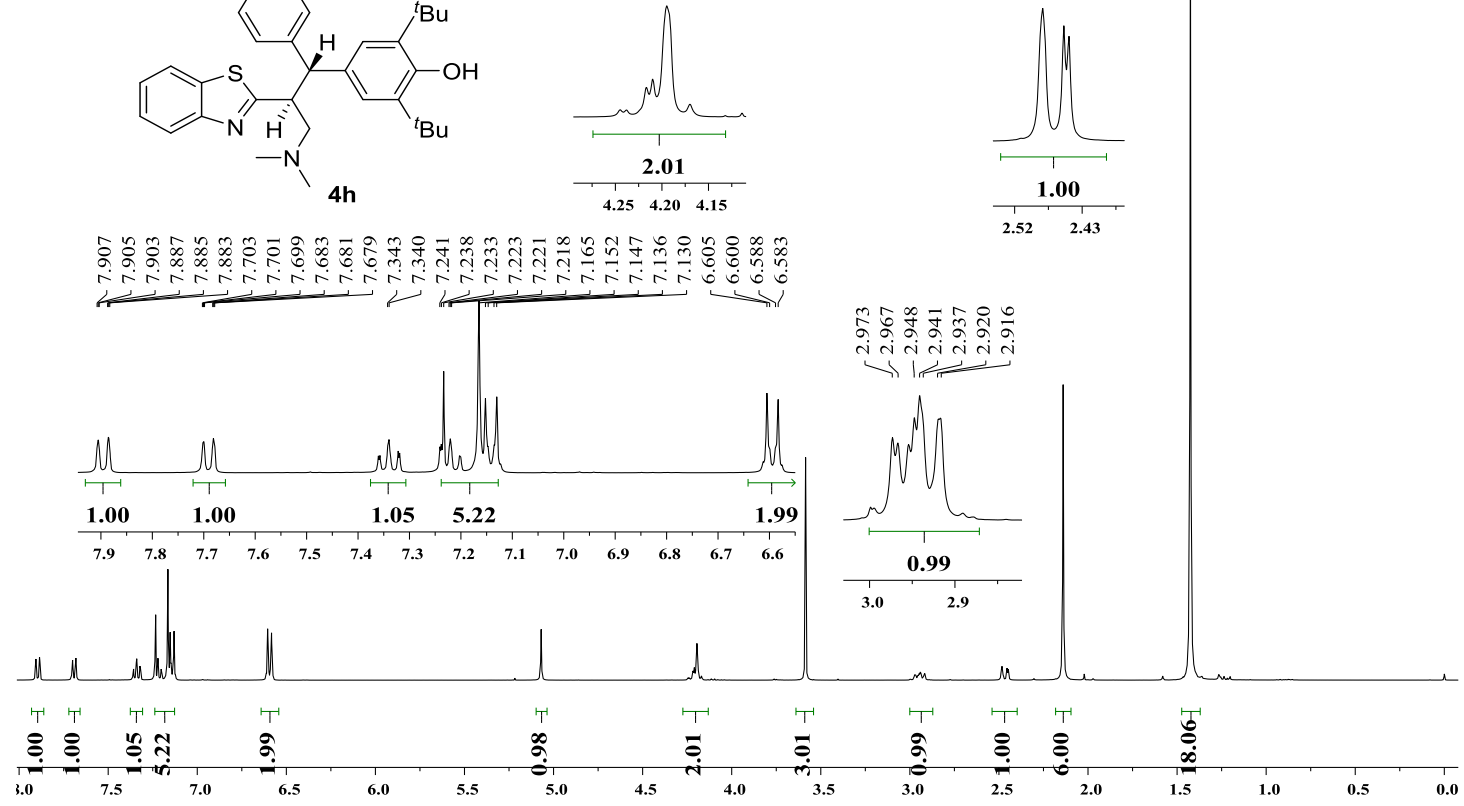

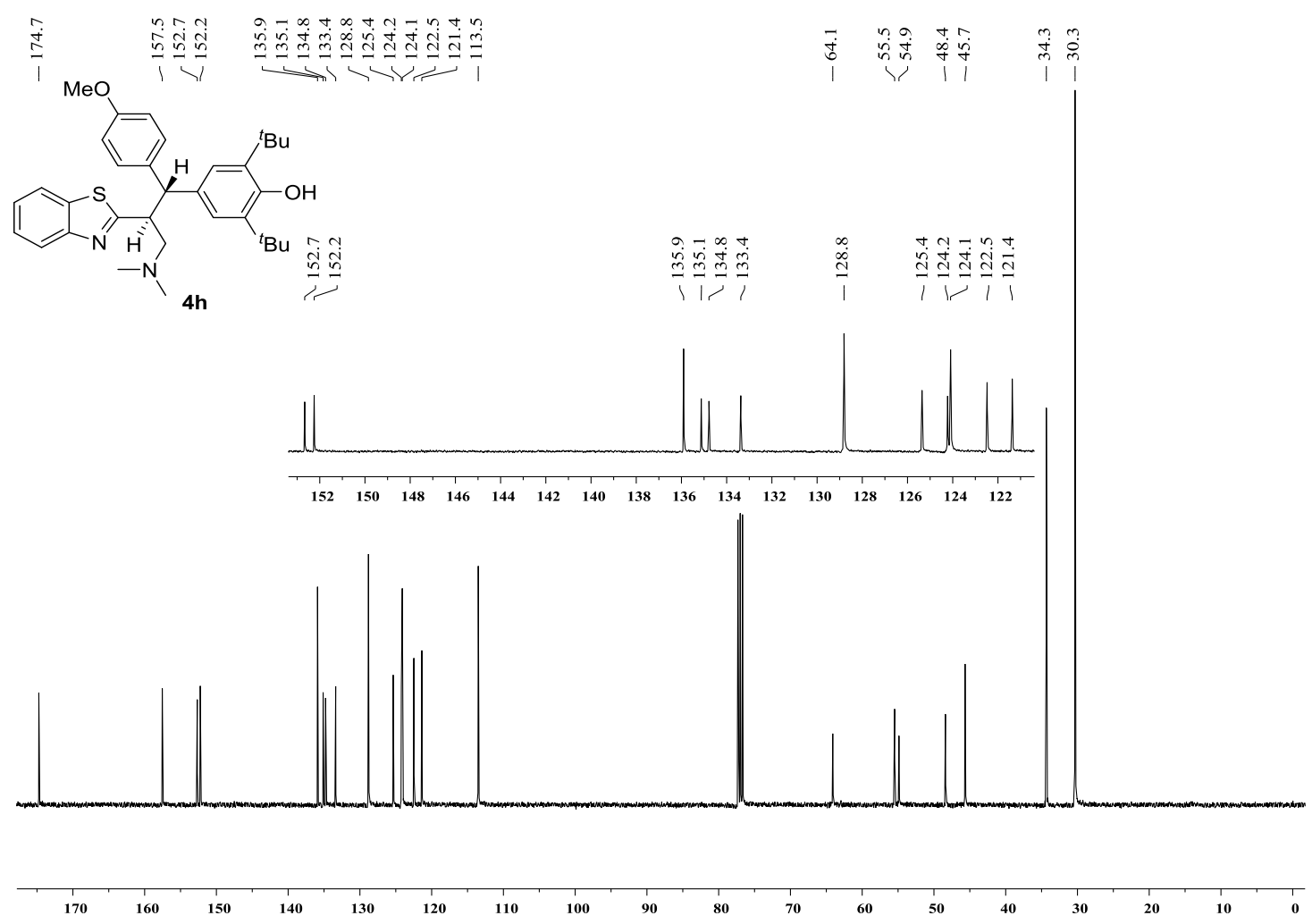

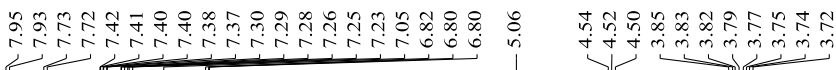
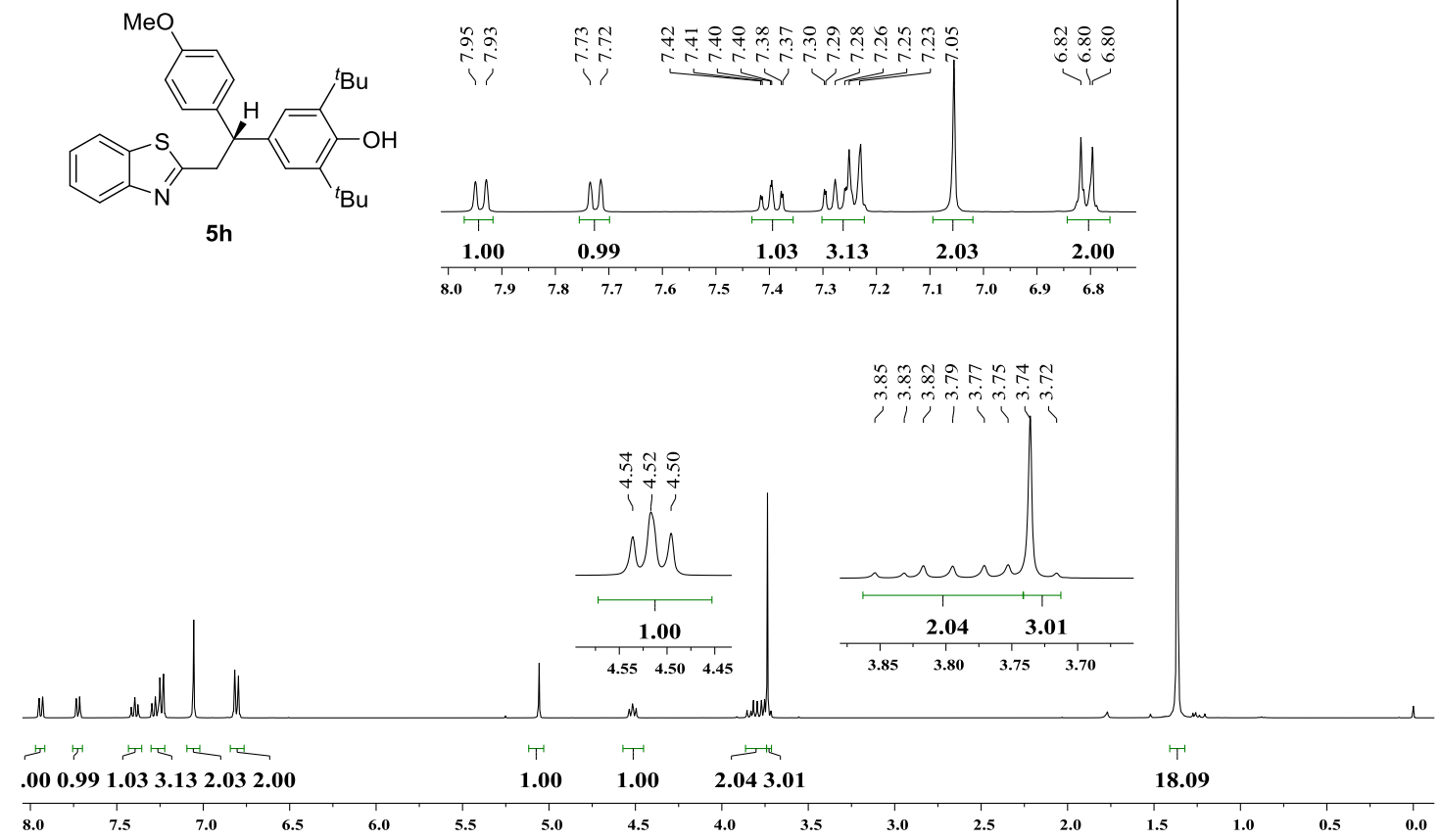


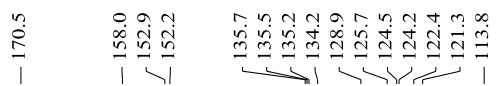

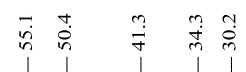

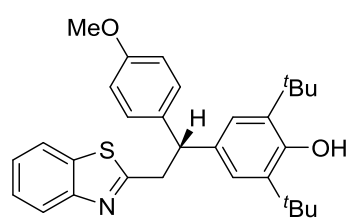

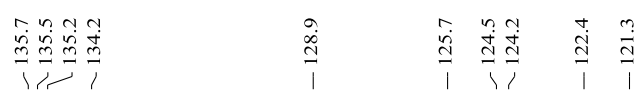

$5 \mathrm{~h}$

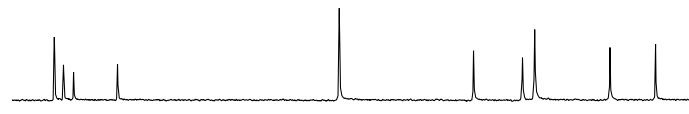

\begin{tabular}{llllllllllllllllllll}
\hline 136 & 135 & 134 & 133 & 132 & 131 & 130 & 12 & 128 & 127 & 126 & 125 & 124 & 123 & 122 & 121
\end{tabular}

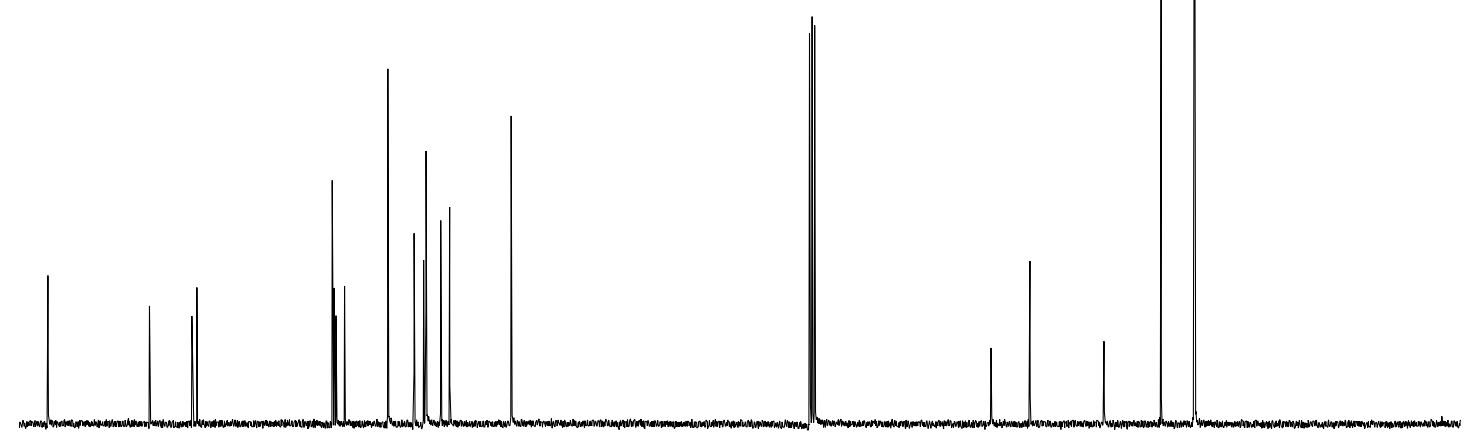

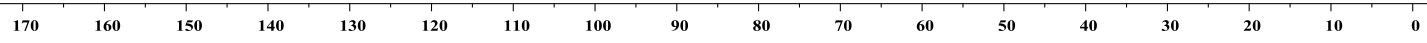

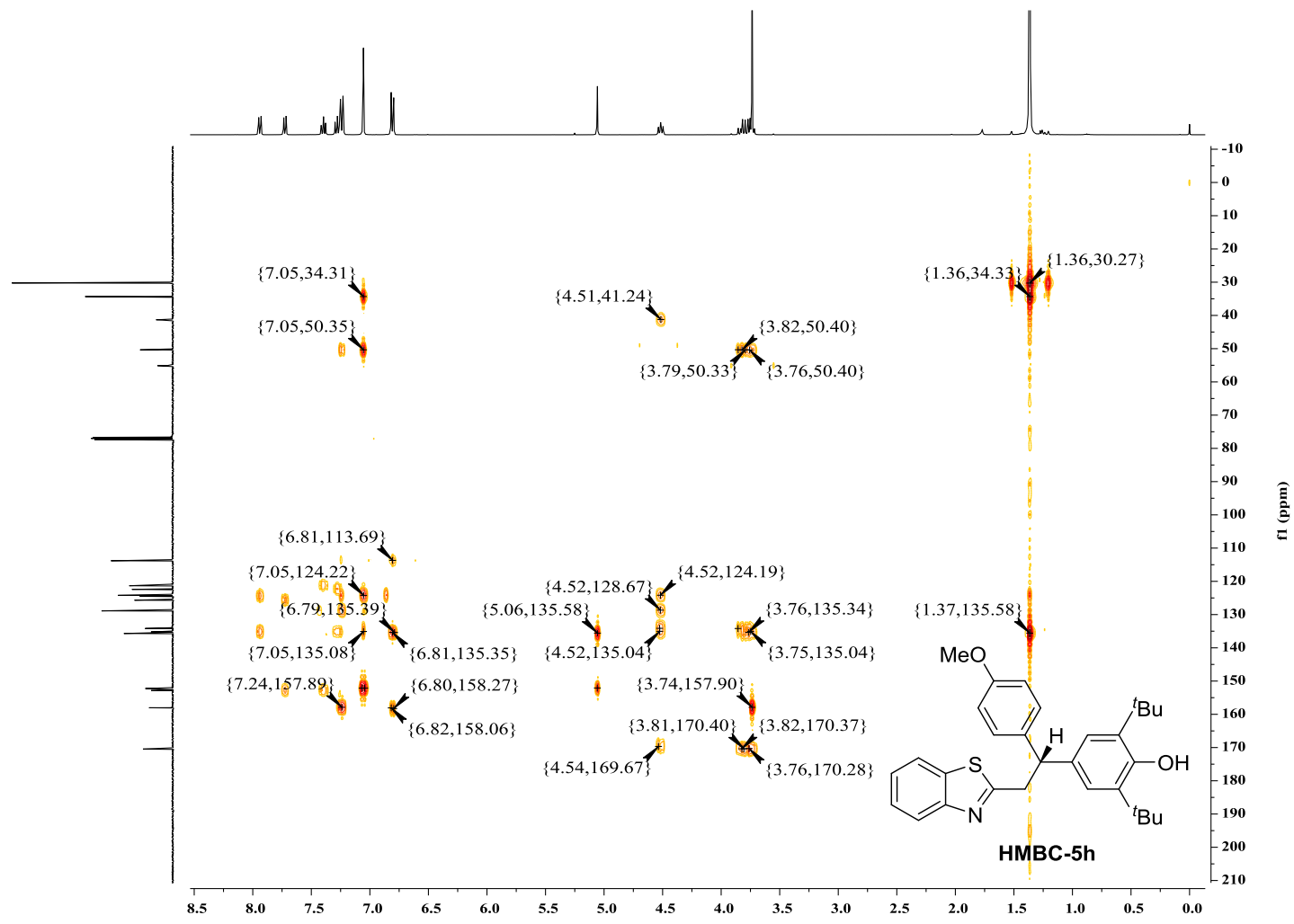



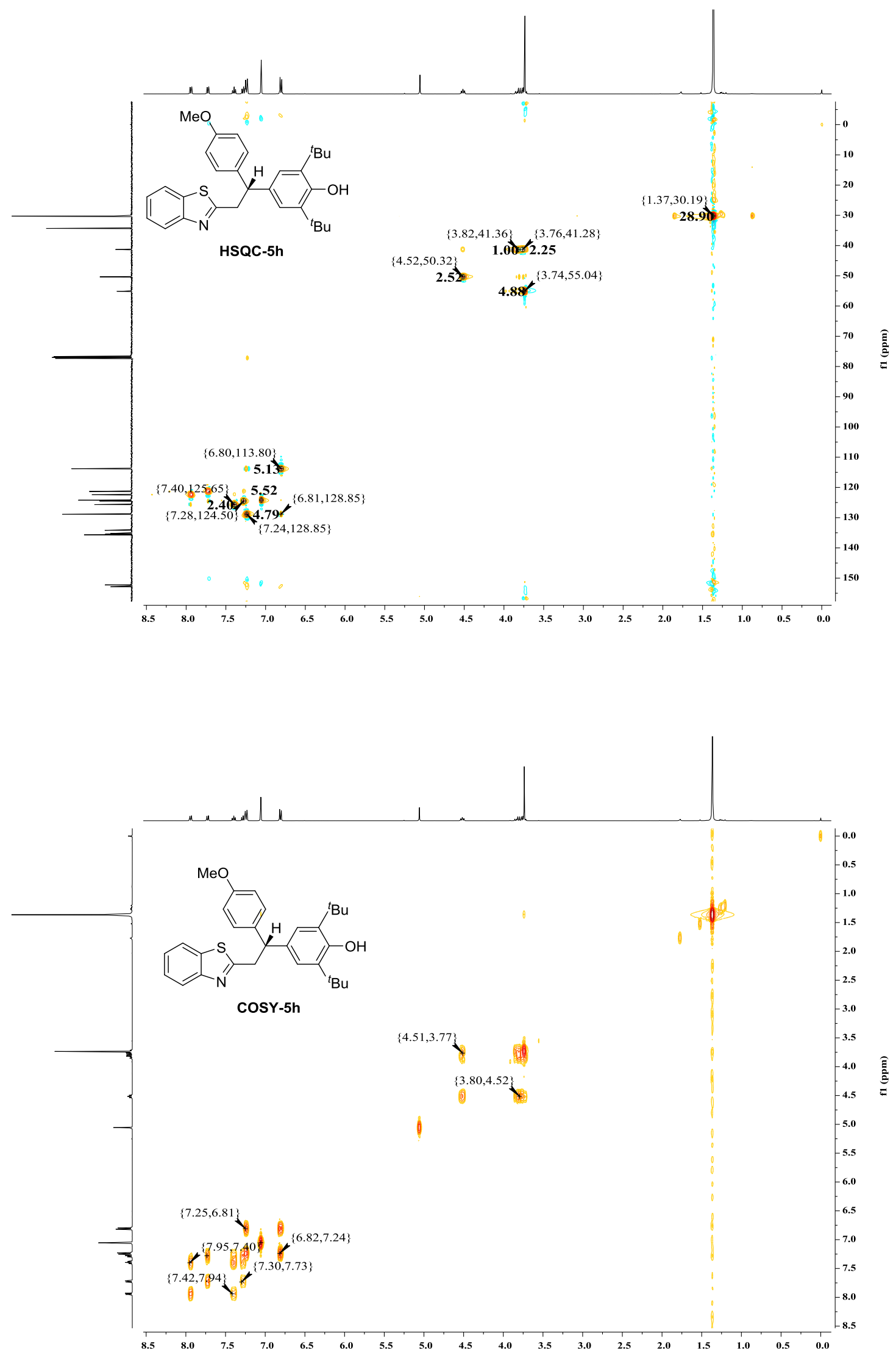